Availability and Deposit of Thesis Form - Open Research Archive

\begin{tabular}{|c|c|}
\hline Author's Name & Amin Rastandeh \\
\hline Thesis Title & $\begin{array}{l}\text { Urban Biodiversity in an Era of Climate Change: Towards an optimised landscape } \\
\text { pattern in support of indigenous wildlife species in urban New Zealand }\end{array}$ \\
\hline Degree Name & Doctor of Philosophy in Landscape Architecture \\
\hline Discipline & Landscape Architecture \\
\hline School & Architecture \\
\hline Supervisor(s) & Dr Maibritt Pedersen Zari and Professor Daniel K Brown \\
\hline Year & 2018 \\
\hline Keywords (3) & Urban biodiversity; Landscape patterns; Climate change. \\
\hline Research Code & 120107 \\
\hline $\begin{array}{l}\text { Availability } \\
\text { Statement }\end{array}$ & $\begin{array}{l}\text { I hereby consent to the above thesis being consulted, borrowed, copied or } \\
\text { reproduced in accordance with the provisions of the Library Regulations from } \\
\text { time to time made by the Academic Board. }\end{array}$ \\
\hline Signature & Amin Rastandeh \\
\hline Date & 17122018 \\
\hline Deposit Declaration & 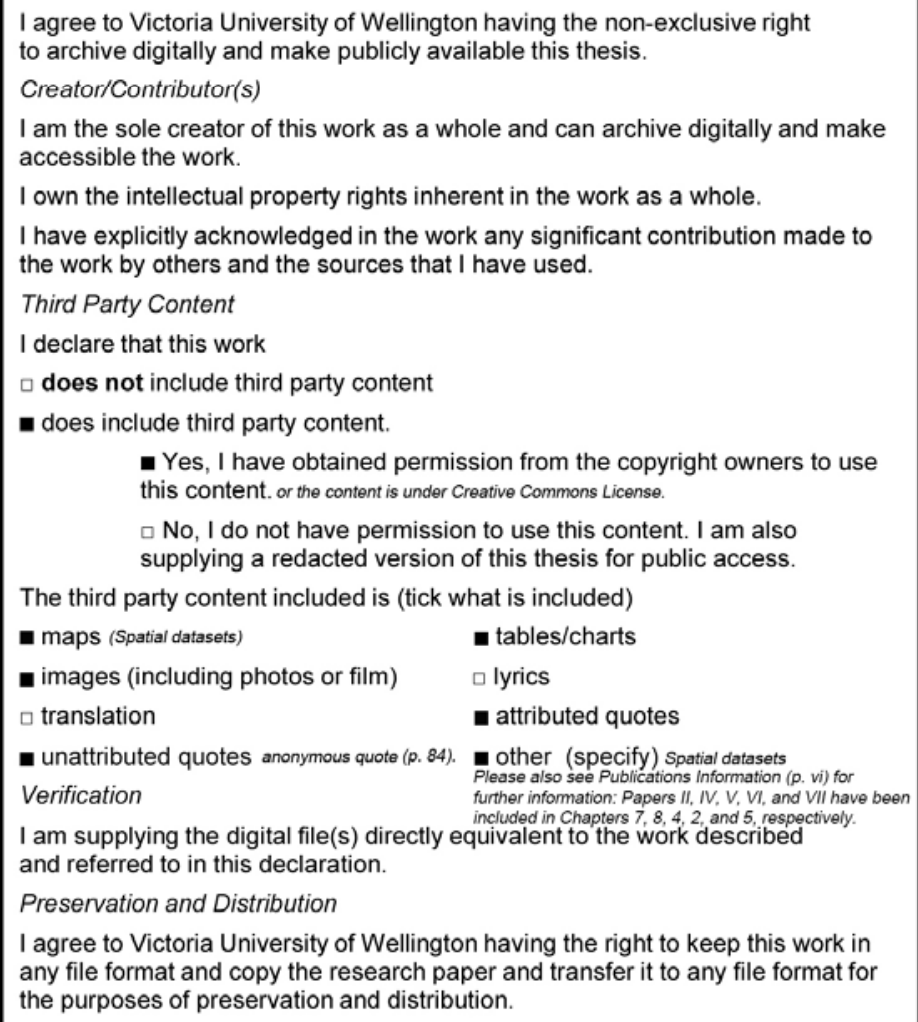 \\
\hline Signature & Amin Rastandeh \\
\hline Date & 17122018 \\
\hline
\end{tabular}




\title{
URBAN BIODIVERSITY IN AN ERA OF CLIMATE CHANGE TOWARDS AN OPTIMISED LANDSCAPE PATTERN IN SUPPORT OF INDIGENOUS WILDLIFE SPECIES IN URBAN NEW ZEALAND
}

BY

AMIN RASTANDEH

\author{
A thesis \\ submitted to the Victoria University of Wellington \\ in fulfilment of the requirements for the degree of Doctor of Philosophy \\ in Landscape Architecture
}





\section{DEDICATION}

To my parents, real teachers in my life, those who helped me to explore and find out my own path towards the future. 


\section{DEDICATION}

To Parisa, a real reflection of 'good thoughts, good words, good deeds'. 


\section{ACKNOWLEDGEMENTS}

Thanks to welcoming New Zealanders, and all people who strive to make this planet a better place for life. Your dreams for having a healthier land, cleaner water, a blue sky with brighter stars, and a more biologically just environment have always been, and will be in my heart. It was the main impetus for me to undertake this research 16,000 kilometres away from my motherland, where I could never forget her thirsty oak forests, lone squirrels, the secret of red pomegranates, and the mysterious silence of ancient ruins.

Undertaking this research would not have been possible without the continued support of others. A three-year scholarship offered by the Victoria University of Wellington's Faculty of Graduate Research enabled me to commence this research in 2015. It was a chance for me to work with three supervisors educated in New Zealand, the United States of America, and England. I am enormously appreciative of my permanent supervisors, Dr Maibritt Pedersen Zari and Professor Daniel K Brown whose feedback has always been supportive and helpful. I am also grateful to Professor Robert Vale who served as my interim supervisor in a short, but critically important stage of my research in 2016.

I am grateful to Dr Mark Johnson for his advice on Human Ethics Approval Applications as well as Dr Lisa Woods for her helpful recommendations on the statistical analysis undertaken in the course of this research.

Eighty-seven researchers worldwide and six New Zealand subject-matter experts contributed to my research through participating in a questionnaire-centred survey (Study I) and in-depth semi-structured interviews (Study II), respectively. Without such a high level of participation, carrying out this research was unlikely.

This thesis has been reviewed and examined by Professor Brenda Vale (Victoria University of Wellington), Professor Penny Allen (University of Technology Sydney) and Dr M. Margaret Bryant (State University of New York). Constructive and supportive feedback received from the examiners enabled me to improve the clarity of my research and reflect a higher level of intellectual merit in this thesis.

My thanks also go to researchers who always responded to my emails when I was still a MSc student in Landscape Architecture (2005 - 2008) and then, as a researcher (2008 - 2015): Professor Tom Turner, Professor Nikos Salingaros, Professor Simon Swaffield, Professor Stephan Pauleit, Professor David Hulse, and Professor Tom Daniels. Feedback and advice from these academics enabled me to gradually crystallise and consolidate my ideas in landscape architecture research in order to start my $\mathrm{PhD}$ research more confidently in 2015. They helped me to develop and improve my own critical thinking in the field of landscape architecture. Special thanks to Professor Kevin McGarigal for giving me permission to provide the audience of this thesis with a verbatim copy of the standard definitions of landscape metrics in Appendix VII (McGarigal, 2015).

I would like to thank the Victoria University of Wellington's Faculty of Architecture \& Design administrative and technical staff for their administrative and technical support during the last three years. Thanks to my colleagues at the extremely cold PhD workstation - WIG001. 


\section{PUBLICATIONS INFORMATION}

Results derived from this $\mathrm{PhD}$ research have been reported in six journal articles and one conference paper. $\mathrm{A}$. Rastandeh conducted data collection/analysis/interpretation as well as formulated the write-up of the present $\mathrm{PhD}$ thesis and prepared the papers derived from it. M. Pedersen Zari and/or D. K. Brown have supervised these processes and provided feedback in the course of the research, when and where required. R. Vale has served as the interim supervisor of the research in 2016 and provided feedback on journal articles V and VII. Written permissions have been obtained from the publishers, as required. In particular, Papers II and VI were reprinted by permission from Springer Nature. Permissions have been obtained from Taylor \& Francis to include Papers $\mathrm{V}$ and VII in the thesis. According to Elsevier's copyright policy, permission was not required for Paper IV. Paper III was written and published during the course of this $\mathrm{PhD}$ research but it was not included in the thesis. The publications listed below have not been cited in the thesis text. To include them in this thesis, where necessary the required modifications have been carried out.

\begin{tabular}{|c|c|}
\hline Publications & \\
\hline Conference paper & \\
\hline $\mathrm{I}$ & $\begin{array}{l}\text { Rastandeh, A., Brown, D. K. \& Pedersen Zari, M. (2017). Biodiversity conservation in urban } \\
\text { environments: a review on the importance of spatial patterning of landscapes. Ecocity World } \\
\text { Summit, 12-14 July, Melbourne, Australia. }\end{array}$ \\
\hline Journal articles & \\
\hline II & $\begin{array}{l}\text { Rastandeh, A., Pedersen Zari, M. (2018). A spatial analysis of land cover patterns and its } \\
\text { implications for urban avifauna persistence under climate change. Landscape Ecology, 33(3), } \\
\text { 455-474. } \\
\text { DOI: [https://doi.org/10.1007/s10980-018-0613-4] }\end{array}$ \\
\hline III & $\begin{array}{l}\text { Rastandeh, A., Pedersen Zari, M. \& Brown, D. K. (2018). Land cover change and management } \\
\text { implications for the conservation of a seabird in an urban coastal zone under climate change. } \\
\text { Ecological Management \& Restoration, 19(2), 147-155. } \\
\text { DOI: [https://doi.org/10.1111/emr.12302] }\end{array}$ \\
\hline IV & $\begin{array}{l}\text { Rastandeh, A., Brown, D.K. \& Pedersen Zari, M. (2018). Site selection of urban wildlife } \\
\text { sanctuaries for safeguarding indigenous biodiversity against increased predator pressures. } \\
\text { Urban Forestry \& Urban Greening, 32: 21-31. } \\
\text { DOI: [https://doi.org/10.1016/j.ufug.2018.03.019] }\end{array}$ \\
\hline $\mathrm{V}$ & $\begin{array}{l}\text { Rastandeh, A., Pedersen Zari, M., Brown, D. K., \& Vale, R. (2018). Utilising exotic flora in } \\
\text { support of urban indigenous biodiversity: lessons for landscape architecture. Landscape } \\
\text { Research, 43(5), 708-720. } \\
\text { DOI: [https://doi.org/10.1080/01426397.2017.1315063] }\end{array}$ \\
\hline VI & $\begin{array}{l}\text { Rastandeh, A., Pedersen Zari, M. \& Brown, D. K. (2018). Components of landscape pattern } \\
\text { and urban biodiversity in an era of climate change: a global survey of expert knowledge. Urban } \\
\text { Ecosystems, 21(5), 903-920. } \\
\text { DOI: [https://doi.org/10.1007/s11252-018-0777-3] }\end{array}$ \\
\hline VII & $\begin{array}{l}\text { Rastandeh, A., Pedersen Zari, M., Brown, D. K. \& Vale, R. (2018). Analysis of landform and } \\
\text { land cover: potentials for urban biodiversity conservation against rising temperatures. Urban } \\
\text { Policy \& Research, In Press. } \\
\text { DOI: [https://doi.org/10.1080/08111146.2018.1551205] }\end{array}$ \\
\hline
\end{tabular}




\begin{abstract}
Biodiversity is the basis of life on the planet Earth. Without biodiversity, ecosystems and the life within them will not thrive. Nevertheless, biodiversity currently grapples with unprecedented challenges attributed to climate change and anthropogenic development, mostly in urban landscapes. While less than 3\% of the world's land surface is covered by urban settlements, biodiversity conservation in urban landscapes is vital because historically most cities have been established at ecosystem junctions where a variety of wildlife species co-exist and interact with abiotic resources to support ecosystem health, and therefore ecosystem services which are essential to human wellbeing, and indeed survival.
\end{abstract}

To support biodiversity and ensure ecosystem services in these human-occupied ecosystem junctions, developing and advancing accurate and reliable knowledge to enable the informed arrangement of ecological patterns and processes in space and time should perhaps be one of the principal tenets of landscape architecture in the twenty-first century. One way this can be fulfilled is through the spatial design of land cover patterns based upon what wildlife require to survive in such a changing and unpredictable atmosphere. Thus, there is an urgent need for undertaking research to inform landscape architecture researchers and practitioners who engage in a wide range of planned interventions in urban landscapes, including decision making on site selection and the allocation of land for human activities or nature preservation, long-term land use planning in its broad sense, urban forestry, landscape restoration, geo-design practices, etc.

In this research, Wellington New Zealand is chosen as the study area. Ecologically fragmented and rapidly growing, the city has been established and continues to expand at one of the most valuable ecosystem junctions in the Southern Hemisphere. As one of the world's most important biodiversity hotspots, New Zealand is experiencing widespread biodiversity loss in its urban landscapes. Unique but fragile, New Zealand indigenous fauna face a wide range of impacts imposed by climate change including ecosystem degradation and habitat loss, biological invasions by some exotic plants, an increased rate of predation by introduced mammals that is exacerbated by rising temperatures, the spread of diseases by introduced species, phenological changes, and food scarcity particularly during winter.

In response to these threats, this research drills down into the role of spatial patterning of patches of vegetation in order to safeguard indigenous fauna against climate change in urban New Zealand where possible. The aim is to examine opportunities for biodiversity conservation through spatial planning and design based upon the habitat requirements of urban fauna as a determinant factor for shaping and characterising urban landscapes. This is conducted to contribute to an informed spatial design of land cover patterns in relation to ecological processes in order to enhance human-wildlife co-existence in urban landscapes and to support the continuation of a wide range of ecosystem services in a climate that continues to change.

A novel methodology employed in this research involves (1) a questionnaire-centred survey of international scholars, (2) semi-structured interviews with New Zealand subject-matter experts, and (3) a GIS-based spatial analysis of Wellington New Zealand using a rich collection of spatial datasets. Arc Map v. 10.4.1, FRAGSTATS v.4.2, and a core set of 15 landscape metrics have been used to quantify and measure the current composition and configuration of land cover classes distributed in Wellington with regards to the spatial ecology of six keystone species endemic to New Zealand.

Results provide an array of land-based information applicable in landscape architecture research and practice. According to this research, the study area has suffered from widespread land cover change and habitat loss over the last two centuries. 
Although a large proportion of the urban landscape is still covered by different types of green space, in most, if not all, cases, the spatial composition and configuration of patches of vegetation do not meet the minimum habitat requirements that will allow urban fauna to respond effectively to the current threats attributed to climate change.

To avoid further biodiversity loss and ensure the natural regeneration of indigenous ecosystems in the region over time, this research suggests that the allocation of land for human activities and/or biodiversity conservation in urban New Zealand should be informed by an in-depth knowledge of the spatial ecology of keystone species, such as kererū and tūi. Based upon this key concept, it is informed interventions in the composition and configuration of land cover classes that are likely to contribute most effectively to safeguarding wildlife species from the local impacts of climate change in urban New Zealand, not necessarily conventional development of green spaces or increasing the percentage of green space per capita without careful strategic consideration of the location and nature of that green space. The nature and level of these interventions should be determined with particular regard to the floristic nature of each land cover class as well as ecological interactions between the land cover classes and urban fauna in space and time. These findings are discussed, depicted, and illustrated in detail and reveal, for the first time, an integrated picture of current capacities and bottlenecks for biodiversity conservation through spatial planning in the context of climate change in urban New Zealand.

The research ends with ten spatially-explicit recommendations for landscape architecture and land use planning practitioners in urban New Zealand, proposing practical solutions for achieving optimised landscape pattern compositions and configurations for safeguarding urban fauna against the impacts of climate change where possible. The research also opens up six specific areas of inquiry for future research in New Zealand and other regions with similar issues and challenges, worldwide.

While the research places particular emphasis on urban New Zealand, lessons learned can contribute to the body of landscape architecture knowledge on a global scale, and show that landscape architects have a critical role in maintaining and increasing the well-being of people in cities through focusing on the health of urban biodiversity. 


\section{TABLE OF CONTENTS}

$\begin{array}{lr}\text { DEDICATION } & \text { iii } \\ \text { ACKNOWLEDGEMENTS } & \mathrm{v} \\ \text { PUBLICATIONS INFORMATION } & \mathrm{vi} \\ \text { ABSTRACT } & \mathrm{vii} \\ \text { TABLE OF CONTENTS } & \mathrm{ix} \\ \text { LIST OF FIGURES } & \mathrm{xiii} \\ \text { LIST OF TABLES } & \mathrm{xv}\end{array}$

CHAPTER 1: Spatial planning for urban biodiversity in an era of climate change 1 1.1. INTRODUCTION

1.1.1. The research philosophy

1.1.2. The importance of biodiversity

1.1.3. Biodiversity in urban landscapes

1.1.3.1. Urbanisation at ecosystem junctions

1.1.3.2. Population growth in urban landscapes

1.1.3.3. Climate change impacts

1.1.4. Biodiversity in the Southern Hemisphere

1.1.5. Biodiversity in urban New Zealand

1.2. RESEARCH STATEMENT

1.2.1. Portraying the current problem

1.2.2. Research aim and question

1.2.3. Literature review

1.2.4. Knowledge gaps in the current literature

1.2.5. Contribution to the body of landscape architecture knowledge

1.2.5.1. Planning for urban biodiversity: spatial perspective

1.2.5.2. A baseline for future research

1.2.5.3. A common language between researchers

1.3. THESIS STRUCTURE

1.4. STUDY AREA

1.4.1. Spatial situation

1.4.2. Predicted impacts of climate change on Wellington

1.4.3. Indigenous species of interest

1.4.4. Documented impacts of climate change on New Zealand indigenous fauna

iii

$\mathrm{V}$

vi

ii

ix

xiii

$\mathrm{XV}$

CHAPTER 2: Study I: A questionnaire-centred survey of international researchers 38 2.1. RESEARCH AIMS

2.2. AN OVERVIEW OF INTERNATIONAL URBAN BIODIVERSITY LITERATURE 38

2.2.1. The state of the art in spatial ecology of urban fauna 38

2.2.2. Urban green space as wildlife habitats $\quad 39$

2.2.3. Urban green space as a human adaptation tool $\quad 39$

2.2.4. Areas of conflict between wildlife and humans: an overview of examples in the literature 40

2.3. MATERIALS AND METHODS $\quad 47$

2.3.1. Questionnaire design and sampling process $\quad 47$

2.3.2. Sample size $\quad 48$

2.3.3. Distribution of the questionnaire $\quad 49$

2.3.4. Geographical distribution of participants $\quad 49$

$\begin{array}{lr}\text { 2.3.5. Statistical analysis } & 50\end{array}$

2.4. RESULTS $\quad 50$

$\begin{array}{lr}\text { 2.4.1. Aggregated information on participants' characteristics } & 50\end{array}$

2.4.2. An expert-driven ranking model $\quad 52$ 
2.4.2.1. Ranking in a global context - Group A

2.4.2.2. Ranking in a Southern Hemisphere context - Group B

2.4.2.3. Ranking in an Oceania context - Group C

2.4.3. Differences within and between groups

2.4.4. Additional components of landscape pattern

2.4.5. Areas of conflict or diverse functioning of urban ecosystems $\quad 60$

$\begin{array}{lr}\text { 2.4.5.1. Question 1: Indigenous vs. exotic } & 60\end{array}$

2.4.5.2. Question 2: Spatial isolation or connection $\quad 61$

2.4.5.3. Question 3: High and low edge density and contrast $\quad 61$

2.4.5.4. Question 4: Spatially compact or dispersed 62

2.5. DISCUSSION $\quad 63$

2.5.1. Ranking components of landscape pattern in the context of climate change 63

2.5.2. Areas of conflict between humans and fauna $\quad 66$

2.6. CONCLUSION $\quad 68$

CHAPTER 3: Study II: Semi-structured interviews with New Zealand subject-matter experts $\quad 70$

$\begin{array}{lr}\text { 3.1. RESEARCH AIMS } & 70\end{array}$

3.2. MATERIALS AND METHODS $\quad 70$

$\begin{array}{lr}\text { 3.3. RESULTS AND DISCUSSION } & 72\end{array}$

$\begin{array}{ll}\text { 3.3.1. Interview questions and responses } & 72\end{array}$

3.3.1.1. Question 1: Components of landscape pattern in a New Zealand context $\quad 72$

3.3.1.2. Question 2: Keystone species under study $\quad 75$

$\begin{array}{ll}\text { 3.3.1.3. Question 3: Potentials for increasing land cover heterogeneity } & 77\end{array}$

$\begin{array}{ll}\text { 3.3.1.4. Question 4. Edge contrast between land cover classes } & 78\end{array}$

3.3.1.5. Question 5: Ranking the local impacts of climate change 80

3.3.1.6. Question 6: Spatial thresholds $\quad 81$

3.3.2. Food availability and pest/weed control $\quad 83$

3.3.3. The role of residential areas in biodiversity conservation $\quad 84$

$\begin{array}{lr}\text { 3.4. CONCLUSION } & 85\end{array}$

CHAPTER 4: Study III: Measuring the spatial extent of land cover classes: indigenous vs. exotic 88

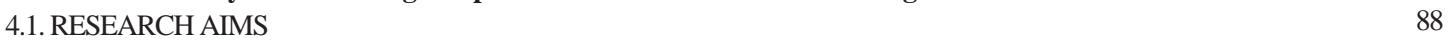

4.2. THE IMPORTANCE OF INDIGENOUS FLORA IN NEW ZEALAND $\quad 89$

4.3. MATERIALS AND METHODS $\quad 91$

4.4. AN OVERVIEW OF THE EFFECTS OF FLORA ON FAUNA $\quad 92$

$\begin{array}{lr}\text { 4.5. RESULTS } & 94\end{array}$

$\begin{array}{lr}\text { 4.6. DISCUSSION } & 96\end{array}$

4.6.1. The importance of scale and resolution $\quad 96$

4.6.2. Opportunities for ecological succession $\quad 97$

$\begin{array}{lr}\text { 4.6.3. Compensatory roles of exotic flora } & 98\end{array}$

$\begin{array}{lr}\text { 4.7. CONCLUSION } & 99\end{array}$

CHAPTER 5: Study III: Landform diversity and indigenousness: a key relationship 101

$\begin{array}{lr}\text { 5.1. RESEARCH AIMS } & 101\end{array}$

5.2. THE IMPORTANCE OF LANDFORM DIVERSITY $\quad 101$

5.3. MATERIALS AND METHODS 103

$\begin{array}{lr}\text { 5.3.1. Study area } & 103\end{array}$

$\begin{array}{lr}\text { 5.3.2. Landform and land cover analysis } & 104\end{array}$

$\begin{array}{lr}\text { 5.4. RESULTS } & 105\end{array}$

$\begin{array}{lr}\text { 5.4.1. Change in urban population, density, and land cover } & 105\end{array}$

$\begin{array}{ll}\text { 5.4.2. South facing slopes as urban climate refugia } & 108\end{array}$

$\begin{array}{lr}\text { 5.5. DISCUSSION } & 111\end{array}$

$\begin{array}{lr}\text { 5.6. CONCLUSION } & 113\end{array}$

CHAPTER 6: Study III: Landscape restoration through natural regeneration mechanisms 115

6.1. RESEARCH AIMS $\quad 115$

$\begin{array}{lr}\text { 6.2. INTRODUCTION } & 116\end{array}$

$\begin{array}{ll}\text { 6.2.1. Seed dispersal mechanisms } & 116\end{array}$ 
6.2.2. Functional connectivity

6.2.3. The ecological importance of the class Gorse and/or Broom

6.3. MATERIALS AND METHODS

6.4. RESULTS

6.4.1. Analysis of the pre-human landscape

6.4.2. Analysis of the current landscape

6.4.3. Topography and the class Gorse and/or Broom

120

6.4.4. Connectivity and distance between seed sources and deposit sites

6.5. DISCUSSION

6.5.1. Natural regeneration in urban landscapes: barriers and opportunities $\quad 125$

6.5.2. A four-step process for evidence-based natural regeneration $\quad 128$

6.6. CONCLUSION

131

CHAPTER 7: Study III: A class-level analysis of the spatial patterning of land cover classes 132

7.1. RESEARCH AIMS 132

7.2. INTRODUCTION

7.3. MATERIALS AND METHODS 133

7.3.1. Consultation with subject-matter scholars 134

7.3.2. Avifauna and climate change impacts 135

$\begin{array}{lr}\text { 7.3.3. Datasets } & 136\end{array}$

$\begin{array}{lr}\text { 7.3.4. Selection of landscape metrics } & 136\end{array}$

7.3.5. Spatial ecology of the selected species $\quad 141$

$\begin{array}{lr}\text { 7.4. RESULTS } & 142\end{array}$

$\begin{array}{lr}\text { 7.4.1. Area/Density/Edge metrics } & 145\end{array}$

$\begin{array}{lr}\text { 7.4.2. Core Area metrics } & 147\end{array}$

$\begin{array}{lr}\text { 7.4.3. Isolation/Proximity metrics } & 148\end{array}$

$\begin{array}{lr}\text { 7.4.4. Connectivity metrics } & 149\end{array}$

$\begin{array}{lr}\text { 7.5. DISCUSSION } & 150\end{array}$

$\begin{array}{lr}\text { 7.6. CONCLUSION } & 156\end{array}$

CHAPTER 8: Study III: Potentials for establishing urban wildlife sanctuaries $\quad 158$

8.1. RESEARCH AIMS $\quad 158$

8.2. INTRODUCTION $\quad 158$

8.3. MATERIALS AND METHODS $r$

8.3.1. Multi-criteria analysis $\quad 159$

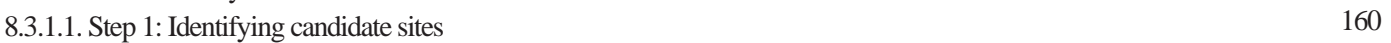

8.3.1.2. Step 2: Determining factors $\quad 162$

8.3.1.3. Step 3: Dedicating weights to factors 162

8.3.1.4. Step 4: Defining attribute values and associated scores for each factor $\quad 163$

8.3.1.5. Step 5: Calculating weighted scores for each factor $\quad 164$

8.3.1.6. Step 6: Calculating composite scores for each candidate site 165

8.4. RESULTS 165

$\begin{array}{lr}\text { 8.5. DISCUSSION } & 170\end{array}$

8.6. CONCLUSION 173

CHAPTER 9: Lessons learned and questions generated $\quad 175$

9.1. INTRODUCTION $\quad 175$

9.2. AN OVERVIEW OF THE KEY FINDINGS 175

9.3. IMPLICATIONS FOR LANDSCAPE ARCHITECTURE $\quad 178$

9.4. RESEARCH LIMITATIONS $\quad 181$

\begin{tabular}{lr} 
9.4.1. Study I & 182 \\
\hline 9.4.2. Sudy II & 182
\end{tabular}

$\begin{array}{lr}\text { 9.4.2. Study II } & 182\end{array}$

\begin{tabular}{lr} 
9.4.3. Study III & 182 \\
\hline
\end{tabular}

9.5. AREAS OF INQUIRY FOR FUTURE RESEARCH 183

9.5.1. Long-term land cover change analysis $\quad 183$

$\begin{array}{lr}\text { 9.5.2. Technology-equipped field survey } & 184\end{array}$

$\begin{array}{lr}\text { 9.5.3. Spatial ecology of exotic fauna } & 184\end{array}$

$\begin{array}{lr}\text { 9.5.4. Predator-prey interactions } & 185\end{array}$ 
9.5.5. Citizen science: an alternative source of information

REFERENCES

APPENDICES

Appendix I: The questionnaire used in Study I

Appendix II: Ethics approval for Study I

Appendix III: Questions addressed in Study II

Appendix IV: Ethics approval for Study II

Appendix V: Land cover classes in the Wellington urban landscape

Appendix VI: Pre-human forest types in the Wellington urban landscape

23

Appendix VII: Landscape metrics used in Study III 


\section{LIST OF FIGURES}

\section{CHAPTER 1}

Figure 1.1

Figure 1.2

Figure 1.3

Figure 1.4

Figure 1.5

Figure 1.6

Figure 1.7

Figure 1.8

Figure 1.9

Figure 1.10

CHAPTER 2

Figure 2.1

Figure 2.2

Figure 2.3
Percentage of urban populations in the world and New Zealand

The timber industry: jacking the rimu logs onto the wagon for transportation to the sawmill, New Zealand

The location of New Zealand's six most populated urban landscapes situated at ecosystem junctions in close proximity with water bodies

The concept of presence, richness, and abundance of fauna in urban biodiversity

Four landscape patterns simulated randomly based upon three sample land cover classes

The geographical location of the Wellington urban landscape (Urban Zone), as the study area in this research, in relation to New Zealand

Wellington, 1858, New Zealand

Examples of biophysical features of the Wellington urban landscape

The concept of pattern process relationship in the literature linked to this research

The relationship between (1) data collection, (2) analysis, and (3) interpretation in this research
4

9

10

13

16

25

26

28

32

An example of areas of conflict between wildlife species and humans

Mean responses for the eight components of landscape pattern in Groups A, B, and C

Comparison of normalised weights assigned to each component of landscape pattern by scientists and researchers in three different groups of World, Southern Hemisphere, and Oceania

\section{CHAPTER 3}

Figure 3.1

Figure 3.2

Figure 3.3

\section{CHAPTER 4}

Figure 4.1

Figure 4.2

Land cover classes present in the study area

Indigenous and exotic land cover classes present in the study area
Scoring scale used to dedicate dissimilarity weights to each pair group of land cover classes

A conceptual illustration of home range size and the movement of avifauna in and between habitat patches (black) in an urban matrix (grey), and reptiles within habitat patches

Examples of edge effects along edges of habitat patches
78

The spatial distribution of patches covered by the classes Indigenous Forest and Broadleaved Indigenous Hardwoods

Figure 5.4 Wellington urban landscape aspects of slopes

108 Hardwoods (right) in the Wellington urban landscape

Figure 5.6

\section{CHAPTER 6}

Figure 6.1

Figure 6.2

Figure 6.3

Figure 6.4

Figure 6.5

Figure 6.6

Figure 6.7

Figure 6.8
Areas covered by the class Gorse and/or Broom at the edge of the Wellington urban landscape Key bio-environmental factors in the process of landscape restoration through natural regeneration Pre-human land cover classes in the Wellington urban landscape Areas of south-facing slopes covered by the class Gorse and/or Broom in the Wellington urban landscape Spatial composition and configuration of the ecological hub in the western part of the Wellington urban landscape in relation to the two classes Gorse and/or Broom and Broadleaved Indigenous Hardwoods

Nearest edge-to-edge distance between patches of indigenous forest in the ecological hub identified in the western part of the Wellington urban landscape

A conceptual spatially-explicit landscape pattern for providing functional connectivity for facilitating species movement and seed dispersal mechanisms between seed sources and deposit sites through stepping stones

A proposed four-step process to support landscape restoration through natural regeneration in urban Southern Hemisphere applicable in landscape architecture 


\section{CHAPTER 7}

Figure 7.1

Figure 7.2

Figure 7.3

Figure 7.4

Figure 7.5

Figure 7.6

Figure 7.7

Figure 7.8

Figure 7.9

Figure 7.10

CHAPTER 8

Figure 8.1

Figure 8.2

Figure 8.3

Figure 8.4

Figure 8.5
The relationship between spatial and non-spatial data and stages undertaken in the research Spatial concepts used in this part of Study III: A: Edge area vs. interior habitat; B: Search Radius; C: Edge Depth vs. Interior Habitat; D: Spatial Isolation; E: Stepping Stones Spatial distribution of plant life-supporting land cover classes in the study area Area/Density/Edge metrics calculated for the 10 land cover classes Core metrics calculated for the 10 land cover classes Isolation/Proximity and Connectivity Metrics calculated for the 10 land cover classes The western part of the Wellington urban landscape as a potential hotspot for urban biodiversity Core area as an important indicator for measuring suitability of urban habitats for wildlife species in an era of climate change

Radius of gyration in the largest patches of indigenous forests in the western part of the Wellington Urban landscape, namely Zealandia Wildlife Sanctuary (I); Otari-Wilton’s Bush (II); Town Belt (III and V); Wellington Botanic Garden (IV)

Core habitats (black) vs. edge areas (grey) measured for 10 land cover classes in the study area

Candidate sites: Town Belt (A), Botanic Garden (B), Zealandia (C), and Otari-Wilton’s Bush (D)

Suitability spectrum used to define attribute values

Land cover classes in the candidate sites: Town Belt (A), Botanic Garden (B), Zealandia (C), and OtariWilton's Bush (D)

Expanses of indigenous flora associated with land cover heterogeneity and landform diversity in Zealandia Expanses of indigenous flora associated with land cover heterogeneity and landform diversity in Botanic Garden

\section{CHAPTER 9}

Figure 9.1

An example of results derived from the overlay analysis revealing applicable opportunities for biodiversity conservation through spatial planning in the western part of the Wellington urban landscape - the ecological hub 


\section{LIST OF TABLES}

\section{CHAPTER 1}

Table 1.1

Table 1.2

Table 1.3

Table 1.4

Table 1.5

Table 1.6

Table 1.7

Table 1.8

CHAPTER 2

Table 2.1

Table 2.2

Table 2.3

Table 2.4

Table 2.5

Table 2.6

Table 2.7

Table 2.8

Table 2.9

Table 2.10

\section{CHAPTER 3}

Table 3.1

Table 3.2

Table 3.3

Table 3.4

Table 3.5

Table 3.6

Table 3.7

Table 3.8

CHAPTER 4

Table 4.1

Land cover classes present in the study area

Thesis structure abridged goals derived from the literature review. fauna Wellington urban landscape
Main components of landscape pattern $\quad 14$

Wellington long-term climate data (1971-2000)

Selected keystone species in urban New Zealand present in the study area $\quad 29$

Documented impacts of climate change on the selected keystone species in New Zealand 31

Main aims, methods and tools in Studies I, II, and III.

Scale of analysis and rationale behind chapters dedicated to report Study III

Landscape pattern analysis in Study III in terms of the most important components of landscape pattern

Main components of landscape pattern composition and configuration, their definitions and rationale used in this research based upon seminal references in landscape ecology

Selected international peer-reviewed publications based upon empirical data and field surveys addressing the role of landscape pattern in affecting fauna in urban landscapes

The role of landscape pattern composition and configuration in support of fauna and humans in the face of climate change including areas of conflict between biodiversity targets and climate change adaptation

Geographical distribution of participants based upon countries in which they have conducted empirical research on the connection between landscape pattern and the presence, richness, and abundance of urban

Expert-driven ranking of the eight most important components of landscape pattern in terms of influence on fauna in an era of climate change in three geographical regions

Differences between mean responses for eight components of landscape pattern in Groups A, B, and C

Mauchly's test of Sphericity performed for Groups A, B, and C

Pairwise comparisons made based upon estimated marginal means for Groups A, B, and C

General information on four open-ended questions addressing areas of conflict in terms of spatial patterning of patches of vegetation in urban landscapes

Statement excerpt depicting the most important and frequently mentioned issues by the participants

Examples of probable consequences of local impacts of climate change on wildlife habitats in the

Interview excerpts depicting the significance of indigenous biodiversity in urban New Zealand

Spatial thresholds proposed for supporting indigenous biodiversity in urban New Zealand linked to components of landscape pattern defined in the current research

The role of non-spatial factors affecting fauna in urban New Zealand and implications for spatial design of land cover pattern interpreted from interviews

The role of residential areas in biodiversity conservation in urban New Zealand

CHAPTER 5

the percentage of each aspect of slope overlapped by the study area, Broadleaved Indigenous Hardwoods (BIH) and Indigenous Forest (IF)

Table 5.3 Percentage of the south-facing slopes overlaid by the two targeted classes compared to the study area 
CHAPTER 6

Table 6.1

The extent of pre-human land cover classes in the Wellington urban landscape

Table 6.2

Current land cover classes in the Wellington urban landscape

121

Table 6.3

Aspects of slope covered by the class Gorse and/or Broom

122

Table 6.4

The eight patches of indigenous forest identified in the ecological hub

CHAPTER 7

Table 7.1

Landscape metrics applied for class-level landscape analysis of the study area's landscape pattern composition and configuration

Table 7.2 Values of the selected landscape metrics for 10 vegetated land cover classes

CHAPTER 8

Table 8.1

Primary criteria for the identification of candidate sites

161

Table 8.2

Total area and percentage of the candidate sites covered by the class Indigenous Forest

161

Table 8.3 Weights dedicated to the eight most important components of landscape pattern

163

Table 8.4

Scoring criteria moderated based upon local conditions applied to dedicate scores to each factor's attribute

164

Table 8.5

Land cover classes consisting of plant life in the candidate sites

166

Table 8.6

Land cover classes identified in the candidate sites

168

Table 8.7

Spatial attributes measured for the candidate sites in terms of the eight factors characterising the spatial

168

Table 8.8 composition and configuration of the candidate sites

Table 8.9

Normalised scores calculated based upon rules defined in Step 5

169

Normalised and weighted composite scores dedicated to the candidate sites in Step 6 


\section{CHAPTER 1 \\ Spatial planning for urban biodiversity in an era of climate change}

\subsection{INTRODUCTION}

\subsubsection{The research philosophy}

Biological diversity ${ }^{1}$, often known as biodiversity, is crucial to support the healthy functioning of ecosystems over time. Ecosystems, in turn, provide a wide range of services for humans. Without biodiversity, ecosystems would collapse and humans would not survive. Nonetheless, continued land cover change caused by anthropogenic development gives rise to ecosystem degradation and habitat loss across the world. This influences biodiversity, accordingly. Climate change $e^{2}$ exacerbates this trend.

In this context, the informed spatial design of landscape patterns is essential to compensate, to a large extent, for the lack of integrated pre-human ecosystems and wildlife habitats. This is to ensure the coexistence of wildlife and humans and to help to safeguard biodiversity against the ill-effects of climate change in urban landscapes, where the rate of human interventions in natural processes is higher than elsewhere and the relationship between abiotic, biotic, and cultural (ABC) resources are not often sustainable in order to support the coexistence of humans and wildlife over time.

\footnotetext{
${ }^{1}$ Biological diversity is "the variability among living organisms from all sources, including, 'inter alia', terrestrial, marine and other aquatic ecosystems and the ecological complexes of which they are part: this includes diversity within species, between species and of ecosystems” (Hawksworth, 1995, 6).

${ }^{2}$ Climate change is "a change of climate which is attributed directly or indirectly to human activity that alters the composition of the global atmosphere and which is in addition to natural climate variability observed over comparable time periods" (UNFCCC, 1992, 7). The accumulation of greenhouse gases (GHGs) produced mainly by human activities is a major driving force behind climate change. GHGs mainly consist of carbon dioxide $\left(\mathrm{CO}_{2}\right)$, methane $\left(\mathrm{CH}_{4}\right)$, nitrous oxide $\left(\mathrm{N}_{2} \mathrm{O}\right)$, and fluoro gases. Since the 1970s, human activities have sharply increased the concentration of these GHGs (IPCC, 2007; 2014). There is evidence that climate change has started from the beginning of the industrial revolution in the second half of the eighteen-century (IPCC, 1995), mainly due to a substantial increase in the burning of fossil fuels. It was in 1896 when the relationship between atmospheric $\mathrm{CO}_{2}$ concentrations and surface temperature was first discovered by Svante Arrhenius, a Swedish scientist in the field of chemistry (Maslin, 2004). In 1975, the term global warming was first used by the geochemist Wallace Broecker in the Journal of Science (Broecker, 1975). In 1979, the first international conference on climate change was held by the World Meteorological Organization in Geneva, Switzerland followed by the establishment of the Intergovernmental Panel on Climate Change (IPCC) in 1988 as a global reaction to climate change impacts. The IPCC's continuing assessment reports concerning different aspects of climate change impacts have demonstrated the depth and extent of this worldwide challenge (e.g. IPCC, 1995; 2007; 2014).
} 


\subsubsection{The importance of biodiversity}

Irrespective of the biotic rights of wildlife to live (Nash, 1989), biodiversity is essential to support the healthy functioning of ecosystems and thereby the persistence and durability of ecosystem services $^{3}$ over time. For example, analysing ca. four-hundred measures of biodiversity effects on ecosystem services, Balvanera et al. (2006) suggest biodiversity has supportive influences on services provided by a wide range of ecosystems. Similarly, the meta-analysis undertaken by Cardinale et al. (2006) shows that biodiversity loss has adverse impacts on the functioning of ecosystems. This fundamental relationship has also been affirmed and documented by others (Hector and Bagchi, 2007; Duffy et al., 2007; Duffy, 2008; Isbell et al., 2011; Hooper et al., 2012; Pasari et al., 2013; Tilman et al., 2014; Lefcheck and Duffy, 2015). Ecosystem services provide humans with a spectrum of services contributing to the wellbeing, health, and ultimately survival of humans (Tzoulas et al., 2007; Carrus et al., 2015; Romagosa et al., 2015; Colley et al., 2016; Southon et al., 2018). The provision of food, energy, and material is the most basic example of ecosystem services.

Ecosystem services also function as natural tools for adaptation to climate change in order to alleviate the magnitude of extreme weather events and concurrently contribute to flood and run-off control, drought management, erosion control, carbon sequestration and storage, wind modification, etc. (Gill et al., 2007; Lin et al., 2012; Schwarz et al., 2012; Abunnasr, 2013; Qiu et al., 2013; Doick et al., 2014; Gillner et al., 2015; Emmanuel and Loconsole 2015; Fernández et al., 2015; Norton, 2015; Wang et al., 2016).

Despite technological advancements, humans cannot replace such nature-driven ecosystem services by human-made artificial systems. Without biodiversity and complex interactions within and between species and ecosystems, human life on the earth will face a real challenge.

\subsubsection{Biodiversity in urban landscapes}

Since 1970, the world has experienced a sharp decline of ca. 52\% in overall biodiversity (McLellan et al., 2014). Urbanisation, inter alia, has been, and is, responsible for a worldwide loss of biodiversity (Section 1.1.3.1). For this reason, biodiversity is an issue of significance in landscape

\footnotetext{
${ }^{3}$ Ecosystem services are classified into four main groups: provisioning, regulating, cultural, and supporting (Millennium Ecosystem Assessment, 2005). The first three groups have direct effects on the quality of human life while the fourth supports other services.
} 
architecture $^{4}$ in urban landscapes (Niemela, 1999; Alberti, 2005; Farinha-Marques et al., 2011; Ahern, 2013; Standish et al., 2013; Ossola and Niemelä, 2017).

Urban landscapes are in part similar to natural areas in terms of ecological processes (McDonnell and Pickett, 1990; Pickett et al., 2013; Forman, 2014). There is a growing body of research on urban biodiversity worldwide heralding a deeper understanding of the importance of urban biodiversity (Müller and Kamada, 2011; Elmqvist et al., 2013; Tan and Jim, 2017). More specifically, urban avifauna hold the key to providing a wide range of ecosystem services that are essential to society including pollination and seed dispersal through flora-avifauna mutualisms (Lepczyk et al., 2017; Murgui and Hedblom, 2017). The aforementioned services, for example, give rise to the long-term ecological integrity of plant communities, ecosystems, and wildlife habitats in urban landscapes (Andersson et al., 2014).

According to the current literature, the importance of urban biodiversity can be discussed with regard to three main issues ${ }^{5}$ : (1) urbanisation at ecosystem junctions, (2) population growth in urban landscapes, and (3) climate change impacts.

\subsubsection{Urbanisation at ecosystem junctions}

Less than 3\% of the world's land surface is covered by urban settlements (CIESIN, 2004). Despite this, biodiversity conservation in urban landscapes is enormously important because urban settlements have often been situated at ecosystem junctions ${ }^{6}$. Ecosystem junctions consist of the meeting points where different types of wildlife habitats overlap (Forman, 1995; Dramstad et al., 1996). This, in turn, creates a biodiverse ecological context for wildlife to interact and coexist. As a consequence, the presence, richness, and abundance of fauna are potentially higher at ecosystem junctions. As abiotic resources (e.g. fertile soil, fresh water, suitable sunlight, humidity, and temperature) are appropriately available at ecosystem junctions (Colding et al., 2003; Kühn et al., 2004; Alvey, 2006), there are suitable conditions for the provision and consumption of food, energy, and materials. Thus, humans have historically been encouraged to colonise these regions to establish human settlements and urban civilisations ${ }^{7}$. The historical outcome of this confluence, however, has

\footnotetext{
${ }^{4}$ In this research, the term landscape architecture is used in its broad sense encompassing a wide range of organised and preplanned interventions in landscapes ranging from green space design in the built environment to large-scale landscape restoration practices (cf. Deming and Swaffield, 2011).

${ }^{5}$ These issues have been identified and classified during the course of the literature review.

${ }^{6}$ These are the meeting points of different ecosystems where various types of wildlife habitats (e.g. forests, wetlands, water bodies, mountains, grasslands, etc.) meet.

7 The Fertile Crescent, the birthplace of urban civilisation, is a historical example of the colonisation of humans for urbanisation at ecosystem junctions.
} 
been a sharp decline in biodiversity worldwide (Dale et al., 2000; Jongman, 2002; Alberti and Marzluff, 2004; Kupfer, 2006; Breuste et al., 2008; McDonnell and Hahs, 2015). This is due to widespread anthropogenic development over time and the lack of enough attention to the importance of wildlife and their interactions with ecosystems as well as the complexity of vital ecological pattern process relationships ${ }^{8}$.

\subsubsection{Population growth in urban landscapes}

Currently, more than half of the world's human population lives in urban settlements (United Nations, 2015). This rate is considerably higher in some specific regions of the world including more developed countries such as New Zealand (Figure 1.1).

Increased urban population growth and dispersed urban development patterns are becoming challenges, worldwide (Abrahamson, 2004; Mulligan and Crampton, 2005; Jones and Douglass, 2008; Bashford, 2014). Between 1950 and 2014, the world urban population has increased from ca. 0.75 to ca. 3.88 billion people (United Nations, 2014). This trend is likely to continue, leading to more threatening conditions for biodiversity worldwide because increasing urban population growth would be associated with a higher demand for land occupation for urban development as well as further consumption and exploitation of natural resources ${ }^{9}$. This, accordingly, may lead to more widespread land cover change, habitat degradation, species extinction, and ultimately biodiversity loss.

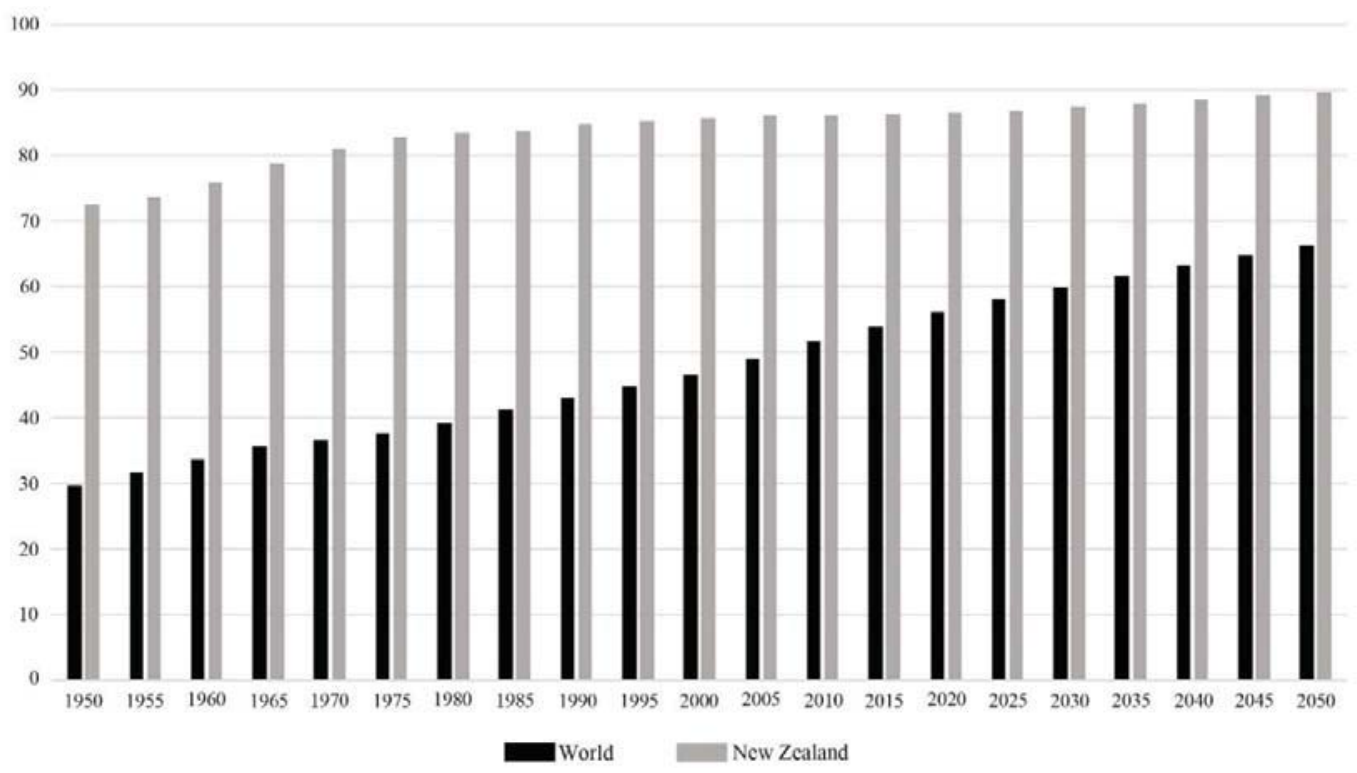

Figure 1.1. Percentage of urban populations in the world and New Zealand - created based upon the world population data (United Nations, 2008; 2014; 2015).

\footnotetext{
${ }^{8}$ Detailed information about the pattern process relationship is provided in Sections 1.1.6 and 1.5.

${ }^{9}$ According to United Nations (2015), up to 6.34 billion people are estimated to live in urban areas by 2050.
} 
As the rate of urban population growth increases, the need for different types of ecosystem services in urban landscapes arises along with the need to tackle a series of environmental challenges that urban landscapes typically face.

\subsubsection{Climate change impacts}

Climate change is one of the most important causes of biodiversity loss, worldwide (Western, 1992; Gitay et al., 2002; McKinney, 2002; Parmesan, 2006; Brook et al., 2008; Berry, 2009; Hoffmann and Sgrò, 2011; Mantyka-pringle et al., 2012; Rosenzweig and Neofotis, 2013; Urban, 2015). Global warming is believed to be a direct threat to biodiversity because the historical evolution of wildlife species has enabled them to live in a specific range of temperatures (Malcolm et al., 2006; Parmesan, 2006). For example, changes in temperature patterns affect the timing of wildlife species' life cycles including breeding seasons (Walther et al., 2002). Predator-prey interactions are influenced by climate change (Broitman et al., 2009; Schmitz and Barton, 2014).

Rising temperatures also can contribute to the spread of infectious diseases amongst fauna (Harvell et al., 2002). Beyond this, the emission of $\mathrm{CO}_{2}$ can contribute to changes in air temperature and precipitation patterns leading to the occurrence of more frequent extreme weather events such as tropical cyclones, hurricanes, and heat waves (IPCC, 1990; 1995; 2014). Scientific simulations show that climate change has led to more extreme El Niño events in recent years (Cai et al., 2014). These environmental disturbances can damage or destroy the structure of ecosystems and wildlife habitats.

In urban landscapes, the ill-effects of climate change can be multifaceted, emerging in various forms including sea level rise, increased drought, flooding, wildfire, landslide, heat island effect ${ }^{10}$, and windstorm (Chamaillé-Jammes et al., 2007; Roaf et al., 2009; Frazier et al., 2010; Hansen, 2010; Logan et al., 2010; Veijalainen et al., 2010; Andersson-Sköld et al., 2013; Liu, 2013; Dugord et al., 2014; Hurlimann et al., 2014; Middelbeek et al., 2014; Saito, 2014; Abdrabo and Hassaan, 2015; Chang et al., 2015; Liu et al., 2015; Luo and Asproudi, 2015; Nam et al., 2015; van Hove et al., 2015). Taken together, such pressures cause habitat degradation, and biodiversity loss in urban landscapes. The coupled pressures of climate change and rapid urbanisation are therefore detrimental to biodiversity. Despite this, as biodiversity can often be rich in urban landscapes (q.v. Section 1.1.3.1), some believe that such a confluence can simultaneously open up new opportunities for humans to tackle, or at least alleviate the impacts of climate change. Grimm et al. (2008), for instance,

${ }^{10}$ Urban heat island effect is not necessarily a consequences of climate change; however, the magnitude and frequency of this phenomenon are influenced by climate change. 
attribute biodiversity loss to rapid urbanisation and climate change. They suggest that both problems and solutions can be found in cities. Similarly, results of ecological modelling of ninety-nine cities in Australia underscore the significant role of urban landscapes in harbouring endangered species (Ives et al., 2016).

If human communities are to improve, or at least maintain, the quality of ecosystem services as the intertwined pressure of rapid urbanisation and climate change impacts increases, healthier ecosystem services will require a higher level of biodiversity to respond to the aforementioned challenges. Therefore, there is an urgent need for a transition from the common response of nice-tohave to must-have when discussing biodiversity in a changing world.

\subsubsection{Biodiversity in the Southern Hemisphere}

Approximately $12 \%$ of the world's human population lives in the Southern Hemisphere (United Nations, 2014), whereas the region covers less than one-third of the world's land surface (Eldredge, 2002). Nevertheless, the rate and density of urbanisation in some parts of the Southern Hemisphere are higher than other parts of the world (United Nations, 2008; 2014). Southern Hemisphere countries also contain a number of the world's most strategic biodiversity hotspots. Fourteen out of the world's twenty-five biodiversity hotspots identified by Myers et al. (2000) are in the Southern Hemisphere. In Oceania in particular, the whole of New Caledonia and New Zealand have been identified as two major biodiversity hotspots (Myers et al., 2000; Olson et al., 2001). Despite a high rate of biological endemism, biodiversity loss as a result of human colonisation since ca. $1600 \mathrm{AD}$ (Diamond et al., 1989) is an ecological challenge unique to many of the Southern Hemisphere's countries, especially island countries such as New Zealand. In addition, some researchers argue that long-term ecological data in Southern Hemisphere countries, even in relatively well-resourced nations including Australia and New Zealand, tends to be poor compared to the Northern Hemisphere (Chambers et al., 2017).

Biodiversity loss resulting from human interventions in Southern Hemisphere landscapes has gradually affected the ecological integrity of the ecosystems. Examples of human-caused biodiversity loss in Latin America (Pauchard et al., 2006; Villavicencio et al., 2016), South Africa (Cowling et al., 1996; van Wilgen et al., 2016), and Oceania (Morton, 1990; Common and Norton, 1992; Craig et al., 2000) are numerous (cf. Elton, 1958). Evidence of the direct relationship between rapid urbanisation and biodiversity decline has been documented in Southern Hemisphere urban landscapes including in Concepción, Chile (Pauchard et al., 2006), São Paulo, Brazil (Brannstrom and Oliveira, 2000), Cape 
Town, South Africa (Rebelo et al., 2011), Sydney, Australia (Mayer-Pinto et al., 2015), and Perth, Australia (Davis et al., 2013).

Unlike the Southern Hemisphere, in Europe, Asia and northern parts of Africa where human beings tend to have concurrently evolved with many wildlife species, almost no human-caused mass extinction has occurred (Diamond et al., 1989). Kingsford et al. (2009) enumerate six threatening processes affecting biodiversity in Oceania and argue that a poor record on species extinction in the region can worsen the current situation.

Developed countries such as Australia and New Zealand are facing ongoing biodiversity challenges related to climate change, but this situation may be even more serious in the Pacific Islands (Jupiter et al., 2014). Recent research shows that rising temperatures are the primary driver of phenological changes ${ }^{11}$ in the Southern Hemisphere in general and in Australia and New Zealand in particular (Chambers et al., 2013). Taylor and Kumar (2016) show that the negative effects of climate change on wildlife species in Oceania’s islands are likely to be significant and widespread.

\subsubsection{Biodiversity in urban New Zealand}

New Zealand is one of the world's most unique biodiversity hotspots (Myers et al., 2000; Olson et al., 2001). More than 86\% of people in New Zealand live in cities (United Nations, 2008) and this figure is estimated to climb to 90\% by 2050 (United Nations, 2014; 2015). As a highly biodiverse and urbanised context, New Zealand is also among those biodiversity hotspots that are more vulnerable to climate change (Bellard et al., 2014). Loss of biodiversity, accordingly, can affect the vast majority of New Zealanders living in cities.

New Zealand has lost a substantial percentage of its unique species since the arrival of people ca. $1000 \mathrm{AD}$ (Tennyson, 2006; q.v. Chapters 4 and 6). Climate change is likely to accelerate this trend (q.v. Section 1.4.4; Table 1.5). Early European immigrants widely developed the timber industry to establish cities across New Zealand (Figure 1.2). At the same time, the arrival of Europeans in New Zealand ca. the 1800s is coincident with the emergence of modern botany and wildlife science in Europe. For example, the first modern textbook on botany entitled Principles of Scientific Botany was published in 1849 (Morton, 1981) and deeply influenced the discipline. Over the nineteenth century, study of organisms in relation to geography and environment became a leading area of research in

\footnotetext{
${ }^{11}$ Biological processes concerning the relationship between the timing of life-cycle events and seasonal climatic patterns are examined in phenology. Phenological changes include seasonal changes in flowering and breeding triggered by global warming.
} 
zoology (Coleman, 1971). Thus, in line with the second wave of human-caused biodiversity loss ${ }^{12}$ in New Zealand associated with land cover change and urbanisation, biological research has been constantly conducted and this, in turn, has provided reliable datasets for contemporary research (cf. Gabites, 1993).

Due to long-term geological isolation for ca. 85 million years (Fleming, 1975), New Zealand's unique indigenous biodiversity ${ }^{13}$ is extremely vulnerable in the face of anthropogenic intervention in ecosystems as well as the impacts of climate change. New Zealand has been separated from other landmasses since around eighty-five million years ago when dinosaurs were widespread on the planet but early mammals (i.e. egg-laying mammal species) were not present on the landmass of New Zealand (Parkes and Murphy, 2003). As a consequence, the long-term evolution of New Zealand indigenous fauna including birds and reptiles has occurred in the absence of mammals for millions of years. Over time, this geo-biological trend has deprived New Zealand indigenous fauna of developing natural defence mechanisms against predation by mammals. Evidence of this ecological uniqueness is emerging. For example, although birds' sense of smell may typically help them to recognise the odour of predators (Amo, 2008; Röder et al., 2016), a study of the ability of exotic and indigenous avifauna to identify mammals' scents in New Zealand suggests that indigenous species cannot respond to predator scent in an effective manner and for this reason, they are more vulnerable to predator pressure from exotic mammals (Stanbury and Briskie, 2015).

Loss of expanses of different types of indigenous forests (i.e. primary habitats of indigenous fauna) has been well documented in urban New Zealand (Clarkson et al., 2007). Although biodiversity loss in New Zealand is nation-wide, the richness of remnant biodiversity in urban landscapes is similar to that found in New Zealand national parks (Given and Meurk, 2000; Meurk, 2005; Meurk and Hall, 2006). This may be due to the fact that most New Zealand cities have been established at ecosystem junctions (Given and Muerk, 2000; Meurk, 2000; Stewart et al., 2004; cf. Section 1.1.3.1) (Figure 1.3).

\footnotetext{
${ }^{12}$ The first wave of human-caused biodiversity loss is attributed to when Māori people came to the country (q.v. Chapter 4).

${ }^{13}$ The term indigenous is deeply meaningful in a New Zealand context and is more culturally appropriate than the term native.
} 


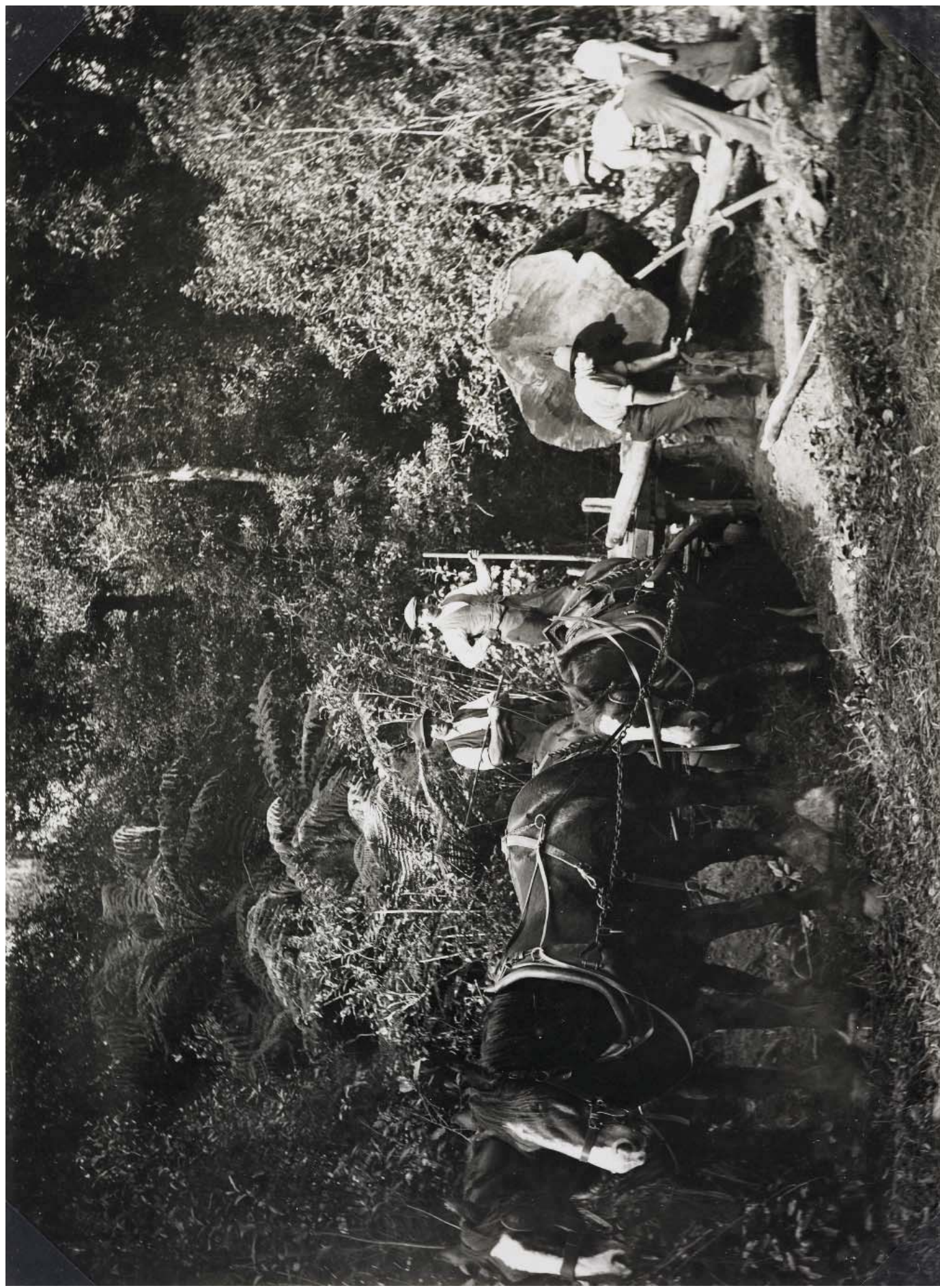

Оิ 


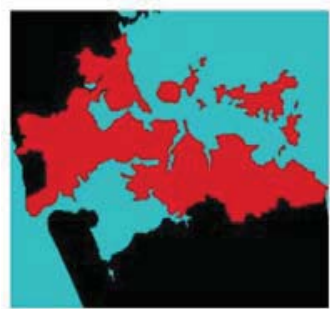

Auckland

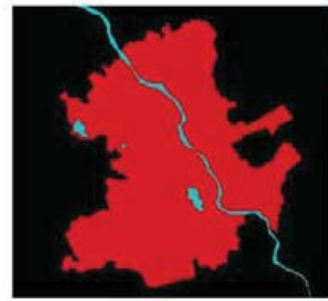

Hamilton

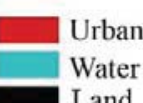

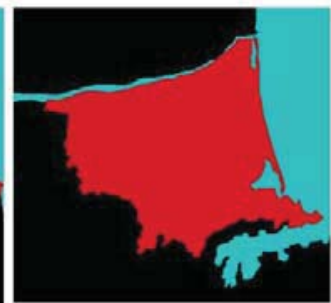

Christchurch

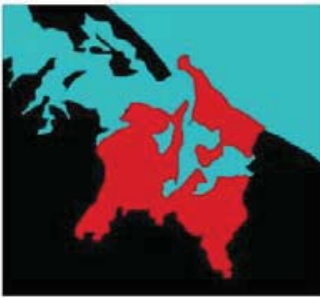

Tauranga

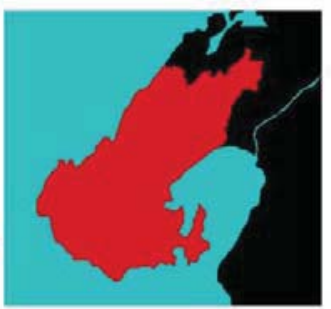

Wellington

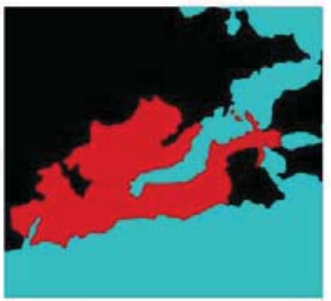

Dunedin

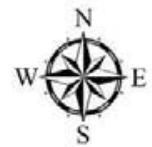

Figure 1.3. The location of New Zealand's six most populated urban landscapes situated at ecosystem junctions in close proximity with water bodies (not to scale).

The introduction of at least thirty-one exotic mammals (Parkes and Murphy, 2003) by humans to New Zealand mainly for commercial purposes has led to widespread indigenous biodiversity loss across the country (McGlone, 1989; Towns and Daugherty, 1994; Saunders and Norton, 200; Ewers et al., 2006). Examples are numerous. Common brushtail possum (Trichosurus vulpecula), for instance, is an Australian mammal introduced to New Zealand in 1837 (Wodzicki, 1950). In Australia, this species plays a vital role in controlling the outbreak of parasites that affect some plant species (Menkhorst and Knight, 2001). In New Zealand, this species is the only introduced mammalian species that potentially serves as a vector to disperse the seeds of some indigenous plants > 10 mm (Williams et al., 2000; Dungan et al., 2002; Williams, 2003). Despite this, the common brushtail possum competes with indigenous fauna for food sources (Innes et al., 2010) and has a serious effect on the life-cycle of some New Zealand plant species such as northern rātā (Metrosideros robusta) (Cowan et al. 1997).

The European hedgehog (Erinaceus europaeus) plays an important role as a dominant pest controller in Europe (Civantos et al., 2012), whereas this species affects populations of some endemic reptiles, wētā, and ground-nesting birds in New Zealand, and therefore, is considered as a threatening pest (Jones et al., 2005; Reardon et al., 2012). The stoat (Mustela erminea) was also introduced to New Zealand to control the population of the European rabbit (Oryctolagus cuniculus), also an 
introduced species, on farms (King, 2017). The stoat's rate of reproduction is very high and it largely predates upon New Zealand avifauna (King, 1983). This can be related to the size of species' home ranges (Murphy and Dowding, 1994). This species widely benefits from the overproduction of the seeds of beech trees during the mast seeding phenomenon ${ }^{14}$ triggered by rising temperatures and this, accordingly, results in a striking increase in the number of stoats leading to greater rates of predation. The weasel (Mustela nivalis) was also introduced for the same purpose. This species is recognised as one of the main predators of indigenous avifauna and reptiles (King, 2017) and is now widespread in urban New Zealand.

The risk of biodiversity loss from climate change in South America, Australia, and New Zealand is higher than other parts of the world mainly due to the impacts imposed by global warming, severity of extreme weather events as a result of El Niño, and pressures imposed by activated exotic mammals under rising temperatures (Urban, 2015; q.v. Section 1.4.4 and Table 1.5). Further biodiversity loss resulting from human interventions in ecosystems as well as the impacts of climate change is very likely in New Zealand (Craig et al., 2000; Gitay et al., 2002; Tennyson, 2006; Christie et al., 2014). A meta-analysis of eighty-nine studies on more than three-hundred species in the Southern Hemisphere with particular concentration on Australia and New Zealand shows an advance in the timing of spring events and consequently the life-cycle of species including breeding seasons (Chambers et al., 2013). This means that phenological changes resulting from global warming are estimated to be very likely in New Zealand (Ridley, 1998; Pryde et al., 2005; Chauvenet et al., 2013; Grayson et al., 2014).

In addition, there exists evidence to show that mosquitoes and some exotic birds are the primary sources and main transmitters of fatal diseases to avifauna caused by some invading parasites (e.g. avian malaria parasites) and infectious diseases (Schoener et al., 2014). Consequently, the spread of such diseases will increase in rising temperatures. This, in turn, increases the mortality rate of New Zealand indigenous avifauna (Baillie and Brunton, 2011; Howe et al. 2012; Ewen et al. 2012; Niebuhr, 2016). For centuries, especially since the 1800s when Europeans came to New Zealand, indigenous biodiversity has been under widespread biological attack across the country by invasive flora and fauna.

Exotic mammals, in particular, are estimated to benefit from rising temperatures because rising temperatures lead to early flowering of flora, and the mast seeding phenomenon providing

${ }^{14}$ By definition, mast seeding is "the intermittent production of large seed crops by a population of plants" (Kelly, 1994, 465). 
more food sources for species. This, in turn, gives rise to a higher rate of reproduction among exotic mammals. This, consequently, increases the risk of increased predator pressures on indigenous fauna in a New Zealand context (Wilson et al., 1998; Pierce et al., 2006; McGlone and Walker, 2011; Christie, 2014). Global warming also contributes to habitat destruction in New Zealand. Extreme weather events such as windstorms and gales give rise to environmental disturbances and habitat loss mainly along edges of habitat patches (Yang and Mitchell, 1984; Davies-Colley et al., 2000). Sea level rise, heavy rainfall, and storms can cause further environmental disturbance and habitat loss (Davis, 1994; Melville and Battley, 2006; Romijn et al., 2012; Ray, 2013).

Current literature indicates that there is a consensus among researchers on the lack of sufficient attention paid to indigenous biodiversity in urban New Zealand (Given and Meurk; 2000; Meurk and Swaffield, 2000; Freeman and Buck, 2003; Swaffield, 2003; Stewart et al., 2004; Freeman, 2005; Meurk, 2005; Meurk and Hall, 2006; Meurk and Swaffield, 2007; Spellerberg and Given, 2008; Ignatieva et al, 2008; Stewart et al., 2009; Doody et al., 2010; Ignatieva et al., 2011; Pedersen Zari, 2012; Meurk et al., 2013; Pedersen Zari, 2015; Meurk et al., 2016). Meurk et al. (2013) enumerate twenty-five services provided by ecosystems relevant to New Zealand urban landscapes. The persistence and durability of these ecosystem services depend, to a large extent, on the quality of indigenous biodiversity. Pedersen Zari (2015) has documented the importance of ecosystem services in an era of climate change in urban New Zealand with particular attention to the key role of indigenous biodiversity (cf. Pedersen Zari, 2012; Ausseil et al., 2013; Meurk et al., 2013).

Although there is not enough evidence in the current literature to provide a detailed picture of what is currently happening to New Zealand indigenous biodiversity as a result of climate change, even the available data is sufficient to raise serious concerns about the possible consequences of biodiversity loss in New Zealand under the changing climate (q.v. Table 1.5).

\subsubsection{The role of landscape pattern composition and configuration}

Conceptual models in landscape ecology science (e.g. the Island Biogeography Model (Mac Arthur and Wilson, 1967); the Source-Sink Model (Pulliam, 1988); and the Patch-Corridor-Matrix Model, Forman, 1995), etc.) provide robust evidence that biodiversity is profoundly dependent on landscape pattern composition and configuration (Forman and Godron 1981; Forman and Godron 1986; Bridgewater, 1988; Turner, 1989; Soulé 1991; Noss and Cooperrider, 1994; Forman, 1995; Murcia 1995; Dramstad et al., 1996; Collinge, 1996; Forman and Collinge, 1997; Savard, 2000; Noss, 2001; Cushman and McGarigal, 2002; Kenny, 2003; Alberti, 2005; Botequilha Leitao et al., 2006; 
Farina, 2006; Opdam et al., 2006; Fischer et al., 2009; Haddad, 2009; Moorcroft, 2009; Heller and Zavaleta, 2009; Morrison et al., 2012; Walz and Syrbe, 2013; Cardille et al., 2017; Loehman et al., 2017). This can be explained under a widely accepted concept in landscape ecology science, often known as pattern process relationship (Forman and Godron, 1986; Turner, 1989; Forman, 1995). This means that characteristics such as the size, shape, distance from or proximity to other patches may have different implications for fauna. According to this concept, landscape pattern affects ecological processes and vice versa at various scales (Bell, 2001).

Landscape pattern, in turn, is constructed and characterised by a number of important biophysical features. In this research, these features are termed components of landscape pattern. The most important components of landscape pattern affecting biodiversity are listed in Table 1.1, as deduced from the literature systematically reviewed in this research (q.v. Section 1.2.3). The first three components are classified under landscape composition, while the latter five are related to landscape configuration $^{15}$. Taken together, the components of landscape pattern construct spatial characteristics of wildlife habitats, either in urban or natural landscapes. Change in any component of landscape pattern, even at small scale, will have different implications for the presence, richness, and abundance of fauna in the scales of space and time (Figure 1.4) ${ }^{16}$. Detailed information about components of landscape pattern is provided in Chapter 2.

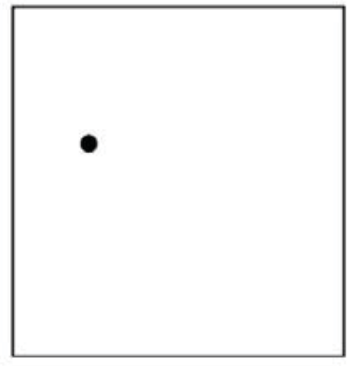

Presence

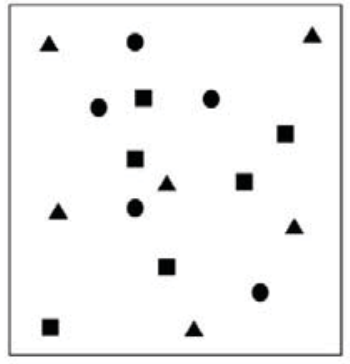

Richness

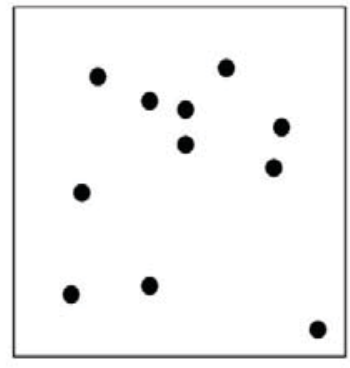

Abundance

Figure 1.4. The concept of presence, richness, and abundance of fauna in urban biodiversity (each shape represents a specific species in the landscape).

The change in components of landscape pattern also has implications for human adaptation to climate change (Zhou et al., 2011; Feyisa et al., 2014; Kong et al., 2014; Maimaitiyiming et al., 2014; Asgarian et al., 2015; Park and Cho, 2016; q.v. Section 2.2.4). Thus, similar to the dependency of

15 By definition, compositional components refer to "the number, type, and extent of [land covers] without explicit consideration of their spatial configuration", whereas configurational components are relevant to "the spatial character, arrangement, position, or orientation” of land covers (Botequilha Leitao et al., 2006, xix).

${ }^{16}$ In this research, presence is defined as the spatial occurrence of a single taxa regardless of the number or diversity of species, richness refers to the diversity of different taxa present in a given site, and abundance is defined in relation to the number of a single taxa. 
fauna on landscape pattern, achieving climate change adaptation goals in support of humans in urban landscapes depends, to a large extent, on the spatial patterning of patches of vegetation in relation to other land cover classes. In terms of spatial patterning of patches of vegetation in urban landscapes, there are, however, some areas of conflict between what wildlife species require and what humans expect from ecosystem services in the context of climate change. Particularly when policymakers seek solutions to adapt urban settlements, infrastructure and people to the local impacts of climate change, the importance of spatial planning of land cover patterns for biodiversity conservation in cities may be underestimated, if not entirely neglected. This is because human activities are an indispensable part of urban landscapes. Therefore, a middle ground should be sought in order for landscape architecture researchers and practitioners to meet both wildlife species and human requirements. This challenge in non-urban contexts is less controversial. In natural or semi-natural landscapes, emphasis is solely placed on wildlife conservation whereas in urban landscapes the process of spatial design of patches of vegetation for wildlife conservation is not typically the main priority where socio-economic forces expect different functions from green spaces ${ }^{17}$. This, consequently, makes this process more complex and multi-dimensional (q.v. Sections 2.2.2 and 2.2.3).

Table 1.1

Main components of landscape pattern (cf. Table 2.1).

\begin{tabular}{|c|c|c|}
\hline \multirow[t]{2}{*}{ Components of landscape pattern } & \multicolumn{2}{|c|}{ Spatial classification } \\
\hline & Composition & Configuration \\
\hline 1. Indigenousness & $*$ & \\
\hline 2. Land cover heterogeneity & * & \\
\hline 3. Land surface perviousness & $*$ & \\
\hline 4. Patch size & & * \\
\hline 5. Connectivity and proximity & & $*$ \\
\hline 6. Edge density and contrast & & $*$ \\
\hline 7. Landform diversity & & $*$ \\
\hline 8. Shape complexity & & * \\
\hline
\end{tabular}

\subsection{RESEARCH STATEMENT}

\subsubsection{Portraying the current problem}

Biodiversity loss resulting from climate change in urban New Zealand is estimated to be widespread and multi-dimensional (q.v. Section 1.4.4; Table 1.5). Widely suffered and eroded over

\footnotetext{
${ }^{17}$ In this research, the term green space is used in its broad sense. It refers to a wide range of patches of vegetation from golf courses to plantation woodlands and indigenous remnant forests in urban landscapes. In this thesis, however, where the ecological nature of green space is of significance, the land cover class name is used instead of the term green space to draw the reader's attention to its ecological characteristics.
} 
the last two centuries, New Zealand indigenous biodiversity is currently experiencing an unprecedented decline (q.v. Section 1.1.5). This, accordingly, will be detrimental to the long-term ecological integrity of the country, will put the country's long-term biosecurity at risk, and influence a series of vital ecosystem services that are essential to support human wellbeing and survival.

Increasing the percentage of green spaces in urban areas is a conventional strategy to support biodiversity and increase the quality level of ecosystem services. Although this strategy may help contribute to urban biodiversity to some extent, the fulfilment of this strategy may be practically unachievable on the ground because the development of urban green space requires enough space, suitable land, and sufficient funds to be designed, implemented, and maintained over time. In addition, not all types of green space provide suitable habitat and food sources for wildlife.

As urban populations expand, open lands should be largely dedicated to housing development and other essential infrastructures to respond to residents' socio-economic needs. This, in turn, contributes to a higher level of landscape fragmentation, ecosystem degradation, and habitat loss. As urban populations increase, this vicious cycle continues. Aside from these socio-economic factors, water shortage resulting from worldwide population growth and global warming may limit the goal of making cities as green as possible, specifically in countries where climate change affects water resources and green space development may lead to greater water shortage (IPCC, 1995; 2014). Therefore, spatial planning for biodiversity conservation in urban landscapes differs, in many aspects, from what policymakers undertake in natural or non-urban areas. Accepting this, different landscape patterns may have different implications for the presence, richness, and abundance of fauna in urban landscapes (Figure 1.5).

In response to this complex situation, an in-depth knowledge of the spatial ecology of wildlife species (i.e. species' behaviours in space and time) may assist urban policymakers to make appropriate decisions on not only the allocation of land to green spaces, but also the spatial design of land cover patterns in a way that meets the habitat requirements of fauna and supports them in urban landscapes, particularly where land availability is widely limited by socio-economic drivers and where human activities are typically given precedence in the process of land use allocation.

In the absence of intact ecosystems in urban landscapes and while socio-economic factors are main players in human-occupied landscapes, there is therefore an urgent need for safeguarding biodiversity against the local impacts of climate change through developing and advancing the knowledge of the pattern process relationship. This kind of ecological knowledge may provide a 
scientific and robust platform for a more informed spatial design of land cover patterns based upon species' behaviours in space and time, when opportunities for increasing the percentage of green spaces are limited. Accomplishing this, an informed arrangement of the existing patches of vegetation can still provide urban fauna with relatively suitable conditions to respond to the local impacts of climate change in an urban context. To achieve this goal, the concept of pattern process relationship should be taken into particular consideration.

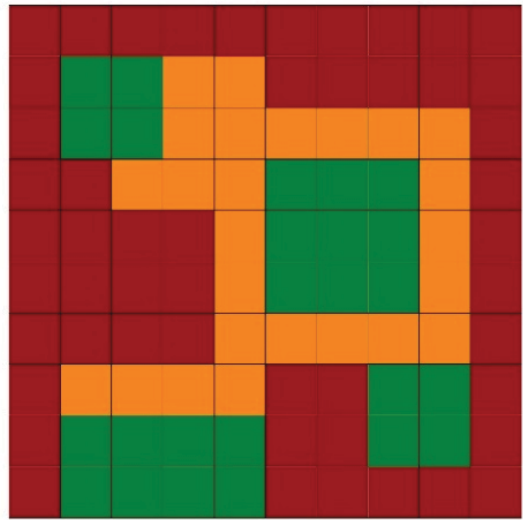

Landscape Pattern I

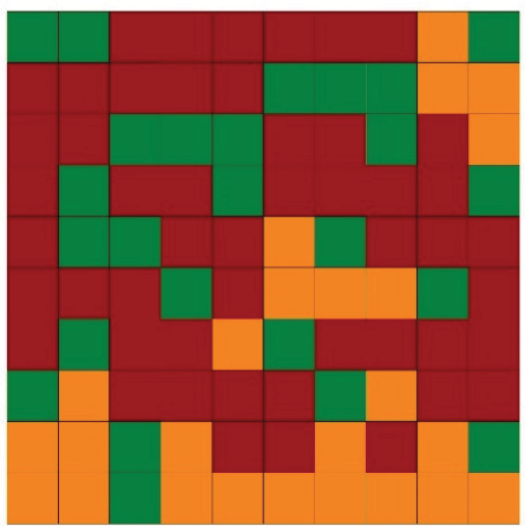

Landscape Pattern III

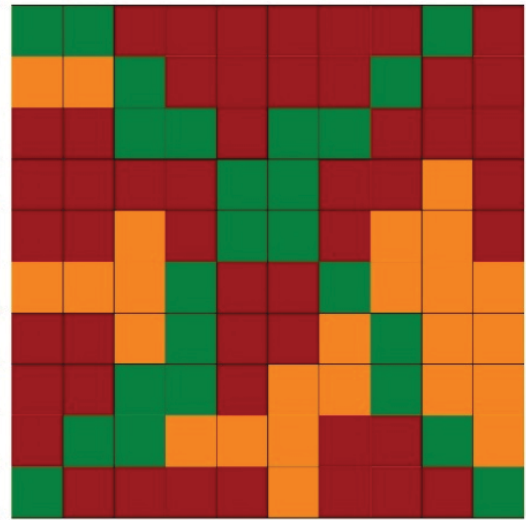

Landscape Pattern II

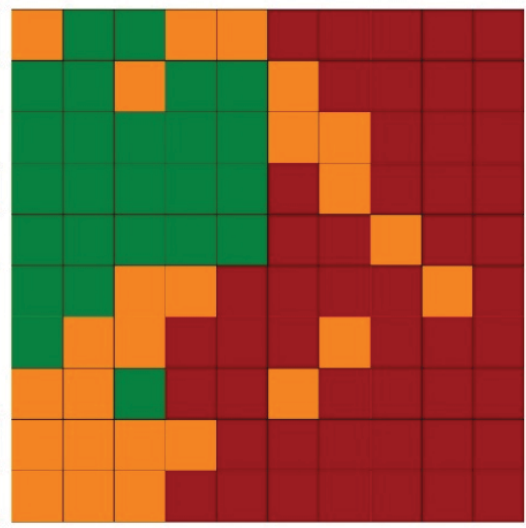

Landscape Pattern IV

Figure 1.5. Four landscape patterns simulated randomly based upon three sample land cover classes: (1) Residential (red), (2) Open (yellow), and (3) Forest (green). Each cell represents 1 ha. The extent of each land cover class is the same in all simulated landscape patterns: (1) Residential (50\%), (2) Open (25\%), and Forest (25\%). Despite this, each landscape pattern has unique implications for biodiversity because land cover patterns differ and this, in turn, will influence behaviours of species in space and time.

\subsubsection{Research aim and question}

The principal aim of this research is to examine and define alternatives for safeguarding indigenous biodiversity against the local impacts of climate change in urban landscapes with particular attention to the relationship between composition and configuration of land cover patterns 
and species' behaviours in space and time. Based upon this aim, the research question is designed as follows:

What is an optimised landscape pattern composition and configuration capable of safeguarding indigenous fauna against the local impacts of climate change in a highly humanoccupied urban landscape ${ }^{18}$ ?

Wellington New Zealand is utilised as the study area to accomplish the research aims and address the research question (q.v. Section 1.4).

\subsubsection{Literature review}

The current literature related to urban biodiversity was comprehensively reviewed using a wide range of available databases including ISI Web of Science, Science Direct, Springer Links, Scopus, Wiley Online Library, and Pro Quest Central. The aim of the literature review was to address the following issues:

(1) To build up an in-depth understanding of pattern process relationship in landscape ecology science using seminal publications in the field;

(2) To develop an understanding of the role of landscape pattern composition and configuration in the presence, richness, and abundance of fauna at the urban scale with particular emphasis on avifauna, small mammals, and reptiles;

(3) To identify the most influential components of landscape pattern that construct and characterise urban wildlife habitats and their influence on the presence, richness and abundance of fauna (q.v. Section 1.1.6).

A wide range of relevant keywords was used to retrieve a large number of publications. Keywords included, but were not limited to, urban biodiversity, urban wildlife, urban bird diversity, urban wildlife management, urban zoology, urban avian diversity, urban avifauna, and fauna. Keywords were searched both separately and cumulatively to find the most relevant published data.

${ }^{18}$ In this research, the term highly human-occupied urban landscape refers to areas where ecosystems have suffered, or are still suffering, from widespread land cover change and landscape fragmentation as a result of human intervention in natural ecosystems. It does not necessarily refer to densely human-populated landscapes. This is because although population density in the study area is very low, the rate of infrastructure creation is proportionately extremely high. Thus, the study area is still considered a highly human-occupied urban landscape. 
Publications retrieved were filtered to gather a limited number of the most preeminent and reliable ones. The Scopus Citation Index and Journal Impact Factors were utilised to examine the quality of publications. Both positive and negative citations were checked for the latest group of publications to ensure that the collection of the selected publications had the highest degree of validity and reliability. Next, the literature review was fulfilled in two stages as follows:

First, given that landscape ecology science confirms that the pattern process relationship is valid in various landscapes and different scales (q.v. Section 1.1.6), seminal publications of international repute on the connection between biodiversity and landscape pattern composition and configuration were explored irrespective of the spatial scale of the studies (i.e. urban or regional).

Second, empirical research on the connection between biodiversity and landscape pattern composition and configuration at the urban scale was specifically reviewed. Geographical diversity of the reviewed research was constantly controlled to ensure that the review considered the global scale and included a diversity of climatic zones. Four criteria were taken into consideration in order to find the most relevant published data in this stage. The publications:

(1) Were peer-reviewed, published, available in English, and indexed on online databases;

(2) Were empirical-based, focused on the connection between landscape pattern composition and configuration and urban fauna’s presence, richness, and abundance on an urban scale;

(3) Received international attention, including a reasonable number of positive citations as recorded on Google Scholar and Scopus;

(4) Represented a local and/or regional picture of the current biodiversity issues (q.v. Section 2.2).

\subsubsection{Knowledge gaps in the current literature}

While the significance of biodiversity in urban New Zealand has been widely underscored by numerous researchers (q.v. Section 1.1.5), research on the spatial dimensions of biodiversity conservation with regard to the local impacts of climate change is still lacking. Research is therefore required to reveal the most important components of landscape pattern in urban New Zealand, and how they may affect the presence, richness, and abundance of indigenous fauna in an era of climate change, particularly considering spatial requirements of urban fauna in relation to flora. 
Furthermore, it is necessary to test if the current landscape patterns have the potential to help safeguard urban fauna against a changing climate. Thus, the spatial composition and configuration of land cover patterns should be quantified and measured to provide a detailed picture of the potential for the conservation of urban biodiversity in the face of climate change in urban landscapes where humans may expect different functions from green spaces (e.g. as a utility for recreational activities or as a tool to mitigate the local impacts of climate change in order to support human health and wellbeing (q.v. Chapter 2). Therefore, a robust research methodology is required to provide a scientific platform for developing and advancing the knowledge of spatial design of land cover patterns in relation to species' behaviours in space and time. This must be done as a way that investigates alternatives for safeguarding indigenous biodiversity against the ill-effects of climate change in urban landscapes while intact ecosystems and integrated wildlife habitats no longer exist.

More recently, the significance of research in this realm has been reemphasised. For example, a review study of the current literature on the relationship between species' behavioural flexibility and climate change impacts highlights an urgent need for developing a deeper understanding of the knowledge of species' behaviours in space and time for biodiversity conservation planning and management in the context of a climate that continues to change (Beever et al., 2017). Despite this, there is still a lack of definite and robust evidence to show responses of urban fauna to the impacts of climate change in the future. Therefore, the Precautionary Principle (Myers, 1993; Cooney, 2004) should be taken into account in order to address the research question ${ }^{19}$. Taking the importance of spatial patterning of patches of vegetation into particular attention, Meurk et al. (2016) argue that New Zealand cannot wait for thorough quantified scientific knowledge while the country's indigenous biodiversity is sharply declining. This means that while empirical ecological data is still widely scattered, lacking or insufficient, continued research is required in order to provide a picture of how spatial patterns may influence the presence and richness of species under a climate that continues to change. In light of this, landscape architecture may be informed as to how to intervene effectively in the existing landscape patterns to enhance the chance of biodiversity conservation in urban landscapes under the impacts of climate change. Thus, the best available information must be gathered and applied to help make more informed, but not necessarily perfect, decisions in the context of landscape architecture.

\footnotetext{
${ }^{19}$ According to the Rio Declaration on Environment and Development (1992): "in order to protect the environment the Precautionary Approach shall be widely applied by States according to their capabilities. Where there are threats of serious or irreversible damage, lack of full scientific certainty shall not be used as a reason for postponing cost-effective measures to prevent environmental degradation" (principle 15).
} 


\subsubsection{Contribution to the body of landscape architecture knowledge}

This research is the first in New Zealand to challenge landscape architecture to designate new roles for researchers and practitioners in the field to respond to biodiversity loss in urban landscapes under a changing climate. In this way, the research contributes to expanding the boundaries of landscape architecture research in three different ways as follows.

\subsubsection{Planning for urban biodiversity: spatial perspective}

Ultimately, alongside a range of spatially-explicit recommendations for biodiversity conservation in urban landscapes through spatial planning under climate change, this research provides a conceptual framework and proposes a novel multi-approach methodology for addressing the capacity of urban landscapes to safeguard biodiversity against climate change impacts, or at least facilitate a higher likelihood of adaptation to impacts. The research quantifies and measures spatial composition and configuration of landscape patterns with regard to species' behaviours in space and time using GIS, FRAGSTATS, and a core set of landscape metrics to provide a robust science-based platform for informed decision-making on land cover patterns in an era of climate change.

While the research focuses on Wellington, New Zealand, the research methodology and findings may also be applied in other urban landscapes in New Zealand, the Southern Hemisphere, or possibly internationally. Results will provide important ecological information for the future spatial design of land cover patterns based upon the relationship between the most important components of landscape pattern and species' behaviours in space and time, a platform for an informed allocation of land to human land use activities, as well as the establishment of urban wildlife sanctuaries in the future while expanses of wildlife habitats are widely confined and engulfed by anthropogenic and climatic factors in, around, and outside urban landscapes. In particular, this research has been undertaken to:

(1) Provide a list of the most important components of landscape pattern affecting the overall level of urban biodiversity in an era of climate change; weight and rank them in terms of importance to urban biodiversity in the context of climate change; address areas of conflict between biodiversity conservation targets and human dimensions of climate change adaptation goals in cities in terms of the spatial patterning of land cover classes distributed across urban landscapes (q.v. Chapter 2); confirm and adjust the results to be applied to a New Zealand context (q.v. Chapter 3); 
(2) Provide the extent of land cover classes and a picture of the current proportion of indigenous and exotic flora in the study area and address the long-term debate of the presence/benefit of indigenous vs. exotic plant species in New Zealand urban landscapes from a spatial perspective (q.v. Chapter 4);

(3) Deepen an understanding of the key role of landform in relation to land cover patterns in spatial planning for biodiversity and reveal spatial opportunities for future biodiversity conservation in the study area (q.v. Chapter 5);

(4) Provide spatial information about the status of land cover patterns in the study area before the arrival of humans and address spatial opportunities for informed landscape restoration practices through natural regeneration mechanisms (q.v. Chapter 6);

(5) Provide a detailed picture of the current composition and configuration of land cover classes containing different forms of plant life in relation to fauna species' behaviours in space and time to examine if the current patterns are capable of safeguarding indigenous fauna against the local impacts of climate change in the study area (q.v. Chapter 7);

(6) Identify, rank, and prioritise the most suitable urban parklands/reserves to be considered as future or expanded pest-free urban wildlife sanctuaries in the face of future climate change impacts (q.v. Chapter 8);

(7) Reveal research areas of critical importance to biodiversity conservation through spatial planning in a world where multiple drivers of change are converging (q.v. Chapter 9).

\subsubsection{A baseline for future research}

Essential spatial data is collected, collated, analysed, and interpreted in the course of this research. This, accordingly, constructs a baseline for undertaking applied research on urban biodiversity in the future. Quantified and measured land cover patterns provide other researchers with basic data for measuring the trajectory of land cover change, changes in the rate and magnitude of habitat provisioning, and the status of indigenous biodiversity as a whole, not only in terms of calculating the percentage of land cover classes, but with regard to measuring the spatial composition 
and configuration of changing patterns using the most important components of landscape pattern as measurable indicators. Spatial research findings and ecological history research are also likely to be useful for researchers and practitioners focused on developing ecosystem services-based approaches to landscape architecture.

\subsubsection{A common language between researchers}

By using GIS as a tool in biodiversity research, the research provides a common language between researchers in different, but related fields of study to address biodiversity issues in space and time. This demonstrates that the landscape architecture discipline can play a more effective role in supporting indigenous biodiversity in the context of a changing climate, significantly beyond the beautification of urban spaces and horticulture between or on buildings and grey infrastructure.

While this research is designed to contribute to the body of landscape architecture knowledge, the potential audience of this research is a wide range of individuals who intervene in landscape patterns in various ways, ranging from local authorities and policymakers to ecologists, spatial planners, and landscape architects ${ }^{20}$.

\subsection{THESIS STRUCTURE}

This thesis is organised into nine chapters to report on three studies (Table 1.2; q.v. Section 1.6). In Chapter 1, the research statement is articulated in detail, and the theoretical framework design is presented and discussed. In addition, Chapter 1 includes a summary of the research methodology. Information about the research materials and methods pertaining to each chapter has been provided in the relevant chapter separately to provide the reader with a detailed and clear picture of the methodology applied in each piece of the research. Studies I and II are reported in Chapters 2 and 3, respectively. Chapter 2 reports a questionnaire-centred survey of international researchers. Chapter 3 reports upon a series of semi-structured interviews with a number of New Zealand subject-matter experts. Both Study I and Study II were designed for data augmentation. Due to the complexity of the issues under investigation, Study III has been reported in Chapters 4 to 8.

\footnotetext{
${ }^{20}$ There is still a widespread misunderstanding about the scope of landscape architecture and the role landscape architects can play in the design, planning, and management of landscapes at different scales. Many researchers have striven to define the boundaries of landscape architecture research and practice to address this misunderstanding (Bruns et al., 2010; Deming and Swaffield, 2011; van den Brink and Bruns, 2014; van den Brink et al., 2017). Although inherently significant, it is out of the scope of this research to address the nature and causes of this misunderstanding.
} 
Chapter 4 is a landscape-level analysis of the extent of land cover classes in the study area. Due to the significance of indigenous biodiversity in the Southern Hemisphere in general, and in New Zealand in particular, in this chapter the question of indigenous vs. exotic flora in urban New Zealand in general, and the study area in particular, is also addressed from a spatial perspective. In this chapter, the study area will be quantified and measured in terms of indigenousness, land cover heterogeneity and land surface perviousness. In Chapter 5, landform diversity is quantified and measured in relation to the current land cover patterns.

Taking the pivotal role of indigenous flora in support of indigenous fauna, a landscape-level analysis is conducted in Chapter 6 to address spatial potentials for landscape restoration through natural regeneration mechanisms in order to examine the potential for ecological succession in the study area. In this chapter, the study area is quantified and measured in terms of connectivity and proximity. In Chapter 7, a class-level analysis of land cover classes is undertaken to quantify and measure the current composition and configuration of patches of vegetation encompassing different forms of plant life. In this chapter, landscape patterns have been interpreted in space and time with regard to the behaviours of relevant keystone avifauna in space and time. In this chapter, the study area is quantified and measured in terms of patch size, connectivity and proximity, edge density, and shape complexity.

Since the presence and survival of a number of New Zealand vulnerable fauna would be very unlikely in the absence of pest-free urban wildlife sanctuaries, in Chapter 8, a patch-level analysis of urban parklands/reserves in the study area is undertaken in order to identify, rank, and prioritise the most suitable sites for the establishment of pest-free urban wildlife sanctuaries in the face of future climate change impacts. This is done using the most important components of landscape pattern as spatial criteria in a suitability analysis. In Chapter 9, an overview of key findings is articulated, research limitations are explained and opportunities for future research are discussed in detail.

While a wide range of publications has been reviewed during the course of the research, no specific chapter was dedicated to a literature review. Instead, current literature was critically scrutinised and cited in each chapter based upon the nature of the topic under discussion. Although the chapters of this thesis have been coherently linked together; each individual chapter has been designed to be read independently, if required. 
Table 1.2

Thesis structure abridged (cf. Figure 1.10; Tables 1.6 to 1.8).

\begin{tabular}{ll}
\hline Chapters & Studies designed \\
\hline 1 & N/A \\
2 and 3 & Studies I and II \\
$4,5,6,7$, and 8 & Study III \\
9 & N/A \\
\hline
\end{tabular}

\subsection{STUDY AREA}

\subsubsection{Spatial situation}

Wellington ( $41^{\circ} 16 \mathrm{~S}, 174^{\circ} 45 \mathrm{E}$ ) is the capital of New Zealand and is situated in the southernmost part of the North Island with a temperate oceanic climate (Table 1.3). The city consists of both urban and rural landscapes (Wellington City Council Open Data, 2015) (Figure 1.6). Widespread land cover change for urban development and agriculture has been occurring in the region since the 1800s (Figure 1.7).

Table 1.3

Wellington long-term climate data (1971-2000) provided by NIWA (2001) ${ }^{21}$.

\begin{tabular}{llllllll}
\hline \multirow{2}{*}{ Rainfall } & \multirow{2}{*}{ Wet days } & \multirow{2}{*}{ Sunshine } & \multicolumn{2}{c}{ Temperature } & & Wind \\
\cline { 3 - 8 } & & & Mean & Very highest & Very lowest & Mean speed & Mean speed $\geq 63 \mathrm{~km} / \mathrm{h}$ \\
\hline $\mathrm{mm}$ & $\geq 1 \mathrm{~mm}$ & hours & ${ }^{\circ} \mathrm{C}$ & ${ }^{\circ} \mathrm{C}$ & ${ }^{\circ} \mathrm{C}$ & $\mathrm{Km} / \mathrm{h}$ & days \\
\hline 1207 & 123 & 1249 & 12.8 & 31.1 & -1.9 & 22 & 22 \\
\hline
\end{tabular}

In this research, the urban zone, termed the 'Wellington urban landscape', was selected as the study $\operatorname{area}^{22}$ (q.v. Figure 1.6). As stated in Section 1.1.5, New Zealand provides a unique platform for research on spatial planning for biodiversity conservation. In particular, the Wellington urban landscape is a Southern Hemisphere example of a city founded at an ecosystem junction (q.v. Section 1.1.3.1; Figure 1.3). Due to the relative richness and availability of biodiversity data, the city can be considered as a platform for urban biodiversity research to address the relationship between landscape

21 Under the Köppen-Geiger climate classification, Wellington falls into the Cfb zone. This means that the city has a temperate climate, without a dry season, and warm summers. According to this classification, the term warm is defined as annual average temperatures between $10^{\circ} \mathrm{C}$ and $22^{\circ} \mathrm{C}$ in summers (Peel et al., 2007).

${ }^{22}$ Although ecological and administrative boundaries are not necessarily the same (Dramstad et al., 1996), administrative boundaries were considered as the boundaries of the study area to increase the productivity and practicality of the research because agencies that are responsible for the implementation of landscape architecture and land use planning practices would be able to utilise and apply the research results under their legal authorities within the boundaries governed by them. 
patterns and ecological processes based upon the conceptual framework designed in this research. The city's rural landscape was excluded from the research ${ }^{23}$.

The study area is estimated to have a population of approximately 203,000 people. This figure is projected to climb to 246,700 by 2043 (Wellington City Council, 2015), equating to ca. 21\% increase in the total number of residents in less than three decades.

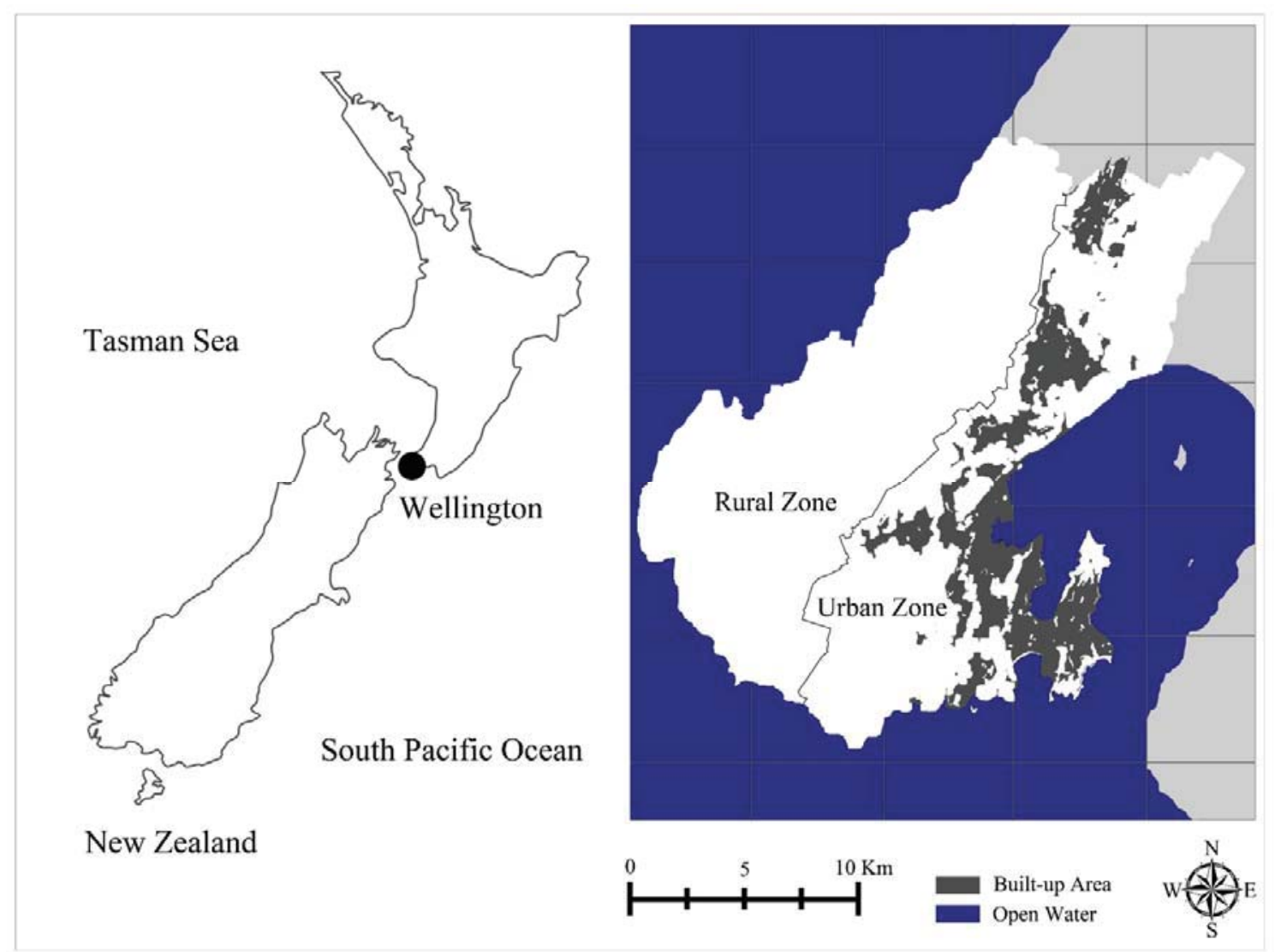

Figure 1.6. The geographical location of the Wellington urban landscape (Urban Zone), as the study area in this research, in relation to New Zealand.

\footnotetext{
${ }^{23}$ There is a marked difference between New Zealand rural and urban landscapes in terms of wildlife species' requirements and the nature of stress on wildlife species imposed by anthropogenic development (Meurk and Hall, 2000; Freeman and Buck, 2003). According to data from Wellington City Council (2015), population density in the rural and urban landscapes is 0.05 to 14.67 people per hectare, respectively. This striking difference helps to justify why the rural landscape should be excluded from the study area. In addition, future urban development in Wellington City is likely to occur within the boundaries of the urban landscape (Wellington City Council, 2015). If managed inappropriately, this trend can give rise to further destruction of urban wildlife habitats.
} 


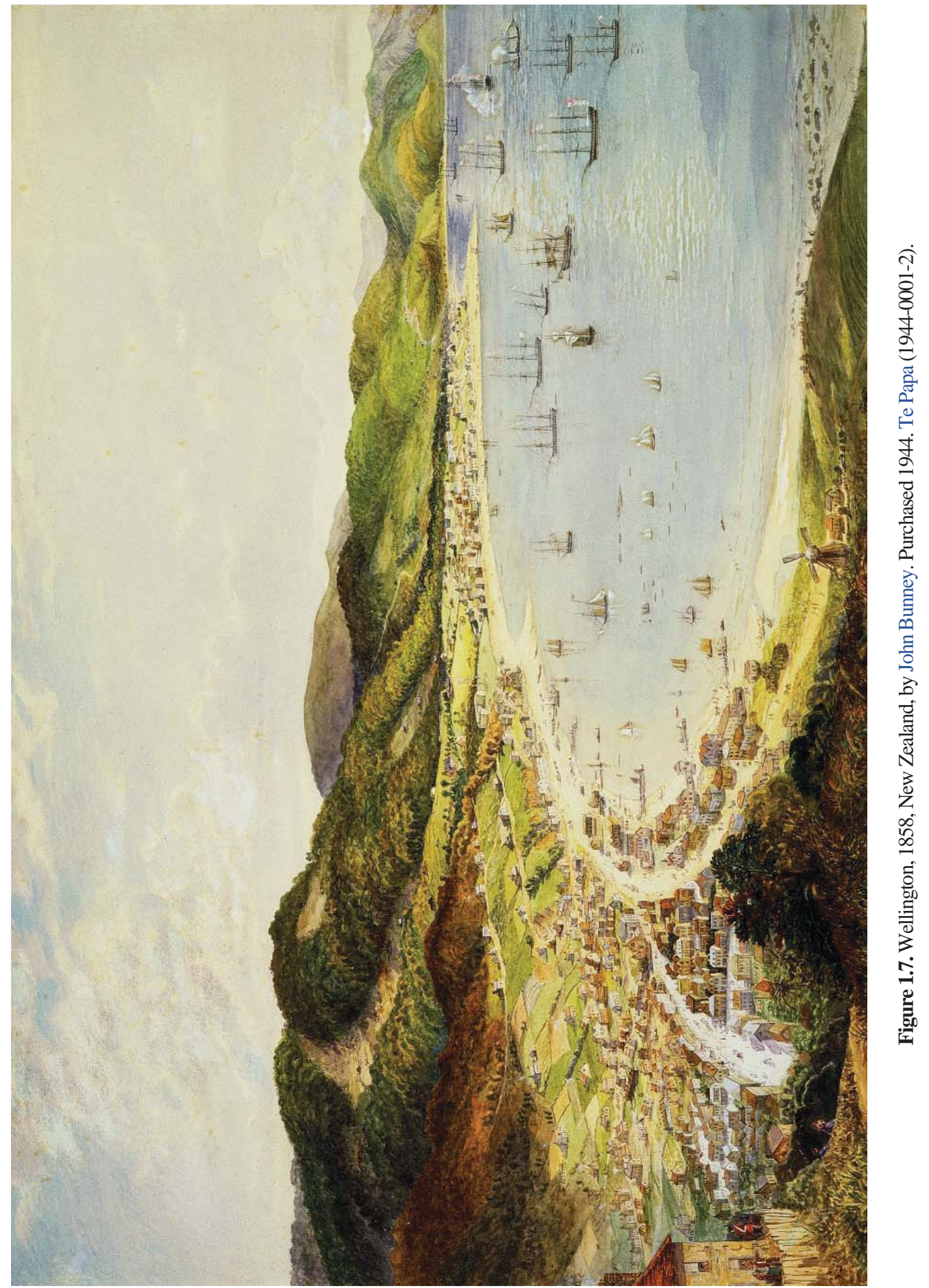


Since the middle of the 1800s, the study area has lost a substantial amount of its indigenous ecosystems (Bagnall, 1979; Pedersen Zari, 2012). The destruction of wildlife habitats (Park, 1999; Towns and Daugherty, 1994) and the introduction of exotic species by humans (Allen and Lee, 2006) have had far-reaching consequences for indigenous biodiversity in the study area during the last two centuries (Clarkson et al., 2007; Pedersen Zari, 2015). Irrespective of biodiversity loss resulting from anthropogenic development, recent research suggests that climate change is leading to further indigenous biodiversity loss (McGlone and Walker, 2011; Christie, 2014). Expanses of patches of vegetation and low-density buildings intertwined with a complex pattern of hills and valleys represent the most important biophysical features of the landscape in the study area (Figure 1.8) ${ }^{24}$.

\subsubsection{Predicted impacts of climate change on Wellington}

According to the Ministry for the Environment (2014), the local impacts of climate change in the Wellington Region are very likely to be:

(1) A $0.9^{\circ} \mathrm{C}$ to $2.1^{\circ} \mathrm{C}$ increase in annual average temperature by 2040 and 2090, respectively compared to 1990;

(2) A $2 \%$ to $5 \%$ increase in the frequency of extreme winds in winter and a decrease by a similar amount during summer;

(3) An increase in the frequency of heavy rainfall events during southerly storms;

(4) An increase and decrease in the number of storms crossing the Tasman Sea during summer and winter, respectively;

(5) A mean sea-level rise of $80 \mathrm{~cm}$ by the end of the century ${ }^{25}$.

\footnotetext{
${ }^{24}$ Detailed information about socio-ecological characteristics of the study area has been provided in each chapter as relevant to the topic under investigation.

25 The figures are based upon middle of the range estimations. In practice, the depth and extent of the impacts are likely to be higher. Thus, it would be crucial to take the concept of the Precautionary Principle into consideration (q.v. Footnote 19) in order to avoid underestimation of impacts on the selected keystone species and consequently indigenous biodiversity in the study area.
} 


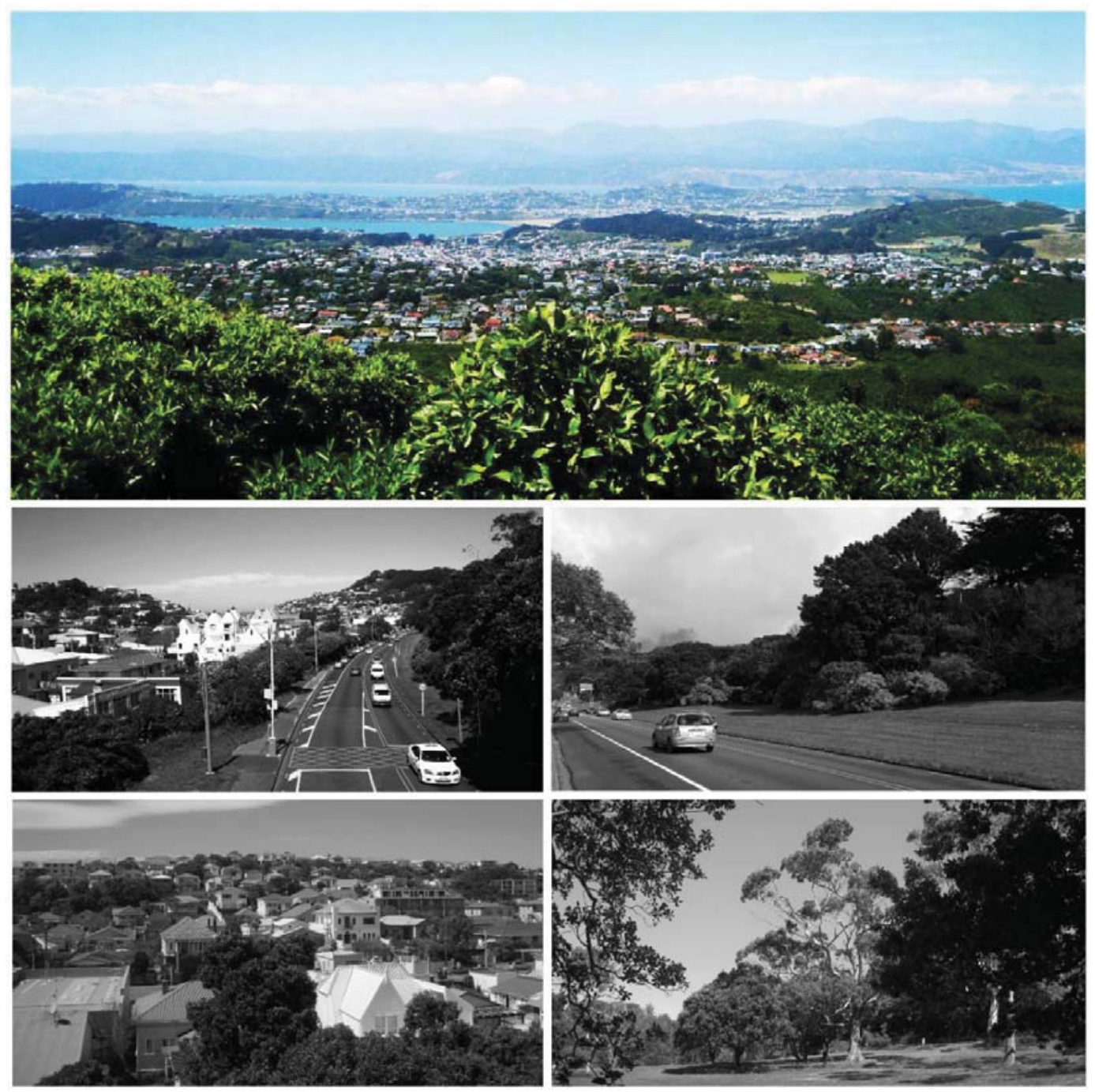

Figure 1.8. Examples of biophysical features of the Wellington urban landscape ${ }^{26}$.

\subsubsection{Indigenous species of interest}

Since landscape ecology, and inevitably spatial planning for biodiversity conservation, is siteand species-specific (Turner, 1989; Ahern, 1999; Botequilha Leitao and Ahern, 2002; Botequilha Leitao et al., 2006), it would be impractical to encompass all species when studying biodiversity in urban landscapes. For this reason, it is reasonable to address a smaller range of keystone species whose roles in urban ecosystems support, to a large extent, the survival of other species that remain outside the study ${ }^{27}$.

\footnotetext{
${ }^{26}$ Images provided in Figure 1.8 do not refer to specific locations of the study area, but provide a general picture of biophysical features of the Wellington urban landscape.

${ }^{27}$ By definition, keystone species are "species whose activities are critical to the structure of the community in which they live” (Collier et al., 1997, 18).
} 
Research shows that the disappearance of keystone species can affect the entire ecosystem over time (Mills et al., 1993). Garibaldi and Turner (2004) argue that species of cultural importance can also be considered as keystone species due to their iconic significance. Payton et al. (2002) identify four types of keystone species relevant to New Zealand, namely organisms controlling potential dominants, resource providers, mutualists, and ecosystem engineers.

In this research, ecological and cultural criteria were also considered to define indigenous keystone species in the study area. Based upon the current literature including Lloyd, 1985; Whitaker, 1987; Clout and Hay 1989; Towns and Williams, 1993; Webb and Kelly, 1993; Bell, 1996; Payton, 2002; Wotton, 2002; Chambers, 2004; Dymond and Shepherd, 2004; Anderson et al., 2006; Boersma, 2008; Foster, 2008; Baber et al., 2009; Anderson et al., 2011; Kunz et al., 2011; Grayson et al., 2014 (q.v. Section 3.3.1.2), an initial list of keystone species present and/or having the potential to be present in the study area was compiled (Table 1.4). These keystone species play a pivotal and irreplaceable role in providing key ecosystem services including the pollination and seed dispersal of indigenous flora. They play a vital role to support natural regeneration mechanisms, food chains, the floristic structure of ecosystems, prey-predation relationships, etc. While a decline in the number of keystone species contributes to widespread urban biodiversity loss, recognising suitable habitats for keystone species may help ensure the overall level of urban biodiversity in the face of climate change.

Table 1.4

Selected keystone species in urban New Zealand present in the study area ${ }^{28}$.

\begin{tabular}{llc}
\hline Keystone species & Scientific name & Endemism \\
\hline Land-based mammals & & Endemic \\
Long-tailed bat & & \\
Indigenous avifauna & & Endemic \\
Bellbird & Anthornismelanura & Endemic \\
Hihi & Notiomystiscincta & Endemic \\
Kererū & Hemiphaganovaeseelandiae & Endemic \\
Little-spotted kiwi & Apteryx owenii & Endemic \\
Tūì & Prosthemaderanovaeseelandiae & \\
& & Endemic \\
Reptiles & & Endemic \\
Forest gecko & Hoplodactylusgranulatus & Endemic \\
Tuatara & Sphenodon & Naultinuselegans punctatus \\
Wellington green gecko & & Native \\
\hline
\end{tabular}

${ }^{28}$ This list was provided after reviewing the literature. The list was revised over the course of the research, based upon lessons learned after the completion of Study II (q.v. Chapter 3). All fauna species, excluding little penguin, are endemic to New Zealand and are not found in other parts of the world. 


\subsubsection{Documented impacts of climate change on New Zealand indigenous fauna}

The local impacts of climate change on wildlife habitats and the selected keystone species are estimated to be diverse, deep, and widespread. From the current literature, likely consequences for the selected indigenous keystone species in New Zealand include, but are not limited to:

(1) Phenological changes in species' life-cycles, specifically seasonal impacts on breeding and flowering affecting respectively fauna populations and food sources upon which they depend;

(2) Environmental disturbances in wildlife habitats' micro-climatic conditions as well as the structure of wildlife habitat resulting from the occurrence of extreme weather events;

(3) Increased predator pressure triggered by mast seeding phenomenon in rising temperatures;

(4) The outbreak and spread of diseases amongst avifauna resulting from an increase in the population of sources of infections triggered by rising temperatures.

Scientific evidence to show how the local impacts of climate change may influence the selected keystone species in the New Zealand context has been classified (q.v. Table 1.5); however, the available scientific methods and tools still cannot truly quantify and measure the long-term impacts of climate change on the selected keystone species. For this reason, applying the concept of the Precautionary Principle is essential to increase the credibility and reliability of decisions and strategies for biodiversity conservation in an era of climate change (q.v. Footnote 19). 
Table 1.5

Documented impacts of climate change on the selected keystone species in New Zealand.

\begin{tabular}{|c|c|}
\hline Keystone species present in the study area & Impacts attributed to climate change in the scientific literature \\
\hline $\begin{array}{l}\text { Land-based mammals } \\
\text { Long-tailed bat }\end{array}$ & $\begin{array}{l}\text { - Foraging source shortage in warmer winters when the species is } \\
\text { likely to be more active than in climatically ordinary winters } \\
\text { (Pryde et al., 2005). } \\
\text { - Predator pressure due to mast seeding phenomenon triggered by } \\
\text { rising temperatures (McGlone and Walker, 2011; Christie, 2014). }\end{array}$ \\
\hline $\begin{array}{l}\text { Indigenous avifauna } \\
\text { Bellbird, Hihi, Little-spotted kiwi, Kererū, Tūî }\end{array}$ & 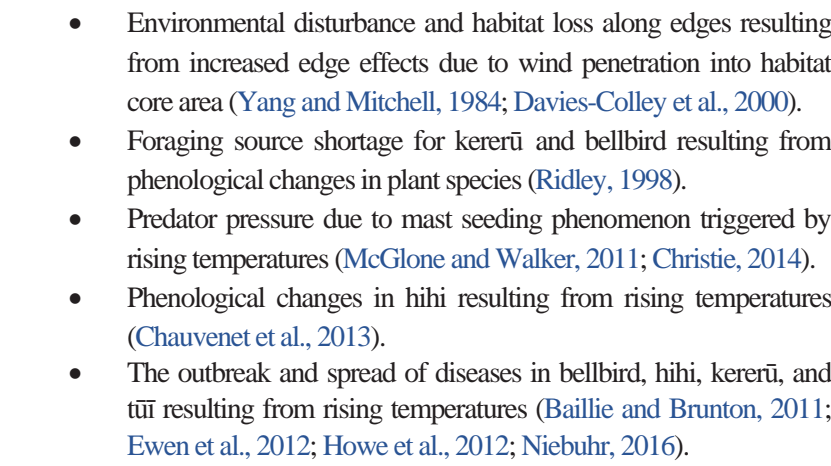 \\
\hline $\begin{array}{l}\text { Reptiles } \\
\text { Tuatara }\end{array}$ & $\begin{array}{l}\text { - Misbalance between male and female populations due to warmer } \\
\text { than average temperatures (Grayson et al., 2014). }\end{array}$ \\
\hline Forest gecko, Wellington green gecko & $\begin{array}{l}\text { - Environmental disturbance and habitat loss due to intensified } \\
\text { - } \quad \text { inds and heavy rainfall (Romijn et al., 2012). } \\
\text { Predator pressure due to mast seeding phenomenon triggered by } \\
\text { rising temperatures (McGlone and Walker, 2011; Romijn et al., } \\
\text { 2012; Christie, 2014). }\end{array}$ \\
\hline $\begin{array}{l}\text { Land-dependent seabirds } \\
\text { Little penguin }\end{array}$ & 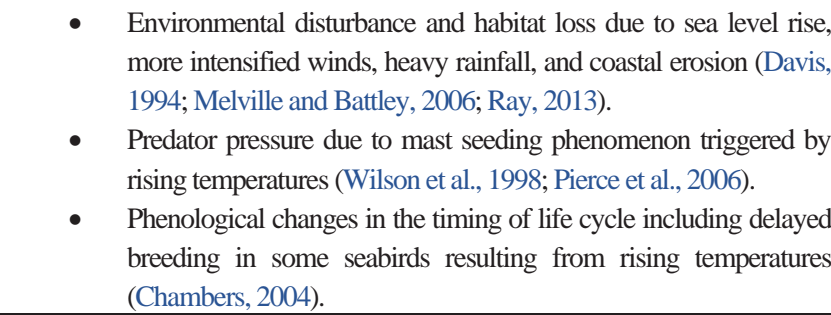 \\
\hline
\end{tabular}

\subsection{THEORETICAL FRAMEWORK}

The research methodology was designed based upon the pattern process relationship (q.v. Section 1.6). There is worldwide evidence to show that this relationship is relevant to urban landscapes (Fernández-juricic, 2001; Cornelis and Hermy, 2004; Garden et al., 2010; Fontana et al., 2011; MacLeod et al., 2012; Strohbach et al., 2013; van Heezik et al., 2013; Chong et al., 2014; Paker et al., 2014; Kang et al., 2015; McClure et al., 2015; Sanz and Caula, 2015; Leveau and Leveau, 2016; Sing et al., 2016; Yuan and Lu, 2016; Jasmani et al., 2017; q.v. Tables 2.1 and 2.2). A conceptual framework was designed to address the research question using a case study of Wellington New Zealand. In this research, patterns and processes are defined respectively by the spatial 
composition and configuration of different land cover classes in the study area and the spatial ecology of the selected keystone species including the species’ behaviours in space and time ${ }^{29}$ (Figure 1.9).

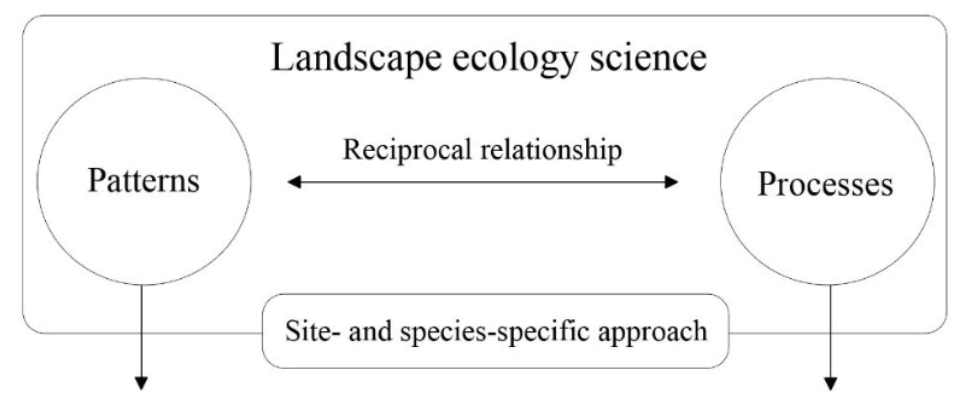

Spatial composition and configuration of land cover type classes in the study area

Spatial ecology of the selected keystone species including species' behaviours in space and time

Figure 1.9. The concept of pattern process relationship in the literature linked to this research.

\subsection{METHODOLOGY SUMMARISED}

This research involves a three-stage process of data collection, analysis, and interpretation to address the research question (Figure 1.10). The research consists of three studies to address the key research question (q.v. Section 1.2.2). The role of Studies I and II was to provide a range of data that is currently unlikely to be found by either reviewing the available literature or field survey. The gaps in the literature can be best understood in tandem with the aims of Studies I and II (q.v. Sections 2.1 and 3.1). For example, to collect data relating to the spatial ecology of the selected keystone species in the study area, there is a need for long-term empirical research to track, observe, and record the behaviour of species in space and time using advanced technological equipment (e.g. GPS, motiontriggered cameras, chew cards, tracking tunnels, etc.).

In terms of funding and time, it was impossible to include this process in this research. Expert-driven knowledge is a reliable source for input data for landscape analysis and GIS-based landscape planning and design decision-making (Baker et al., 2004; Nassauer and Corry, 2004; Jacobs et al., 2015). Although subjective, this is a common approach in landscape architecture research for data collection because it is cost-effective, feasible, and informative (Milburn and Brown, 2003; Deming and Swaffield, 2011; van den Brink et al., 2017).

In Study III, spatial analysis of the study area was undertaken in three levels of patch, class, and landscape utilising different types of spatial and published data as well as that gathered in the

\footnotetext{
${ }^{29}$ Species' behaviours in space and time include, but are not limited to, habitat selection, movement between habitat patches, nesting, roosting, foraging, breeding, etc.
} 
course of conducting Studies I and II (q.v. Chapters 4-8) (Table 1.6). Field surveys including casual and informal observations were also made for data augmentation. The aim was to familiarise the researcher with the study area and ensure the accuracy of spatial data including the extent of land cover classes in certain locations (q.v. Chapters 4 and 8).

Table 1.6

Main aims, methods and tools in Studies I, II, and III.

\begin{tabular}{lll}
\hline Study & Main aims & Main methods/tools \\
\hline Study I & Data collection & A questionnaire-centred survey of international researchers \\
Study II & Data collection & Semi-structured interviews with New Zealand subject-matter experts \\
Study III & Analysis and interpretation & Spatial analysis of landscape patterns using GIS and FRAGSTATS \\
\hline
\end{tabular}



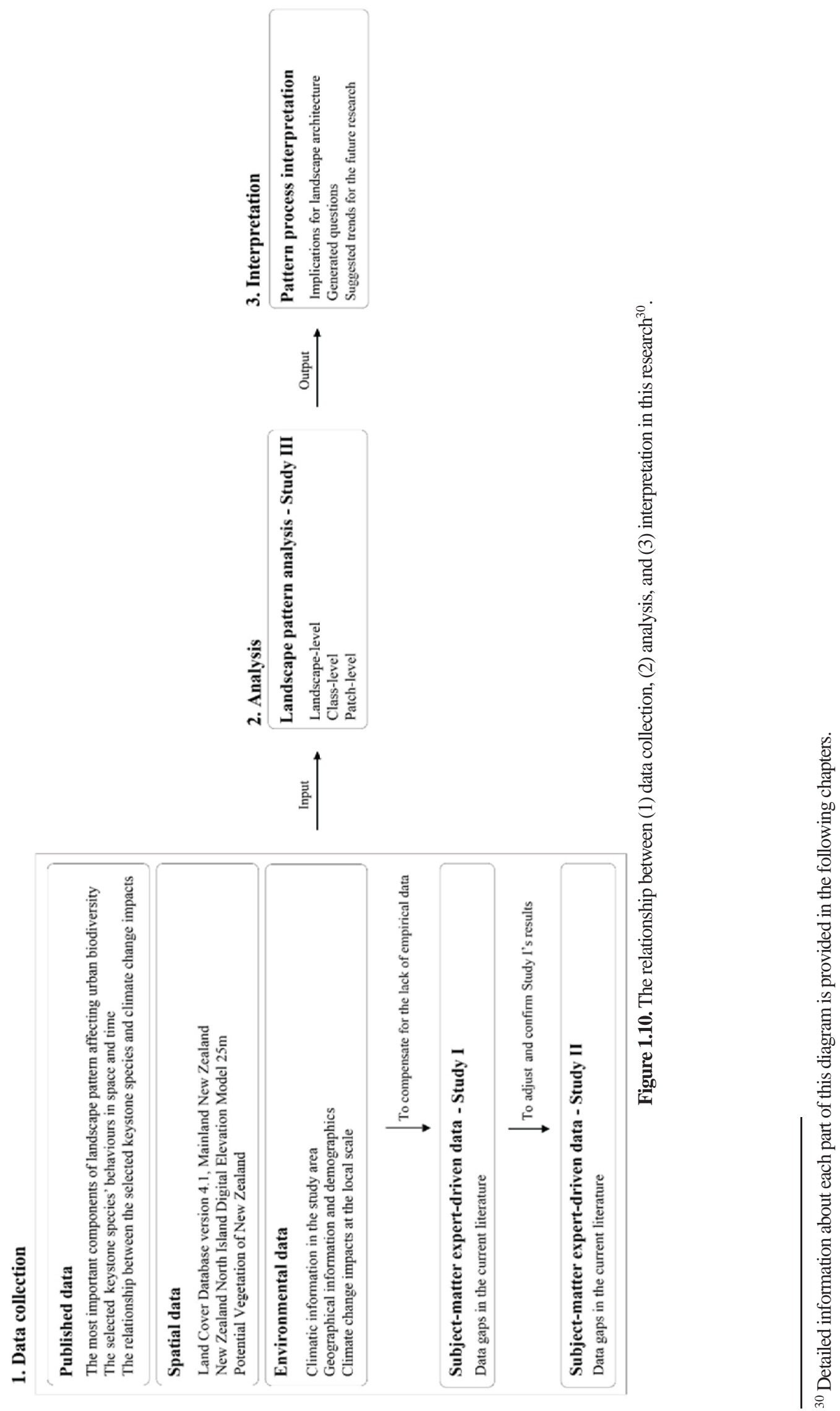


\subsubsection{Study I: A global perspective}

Climate change and biodiversity loss in urban landscapes are two common, ongoing, and converging issues worldwide. Therefore, Study I was designed based upon a questionnaire-centred survey of internationally recognised researchers with track records of empirical research on spatial planning for biodiversity conservation to address how to:

(1) Finalise the list of the most important components of landscape pattern affecting the presence, richness, and abundance of urban fauna biodiversity (q.v. Table 1.1) in an era of climate change;

(2) Weight and rank the most important components of landscape pattern in terms of importance to urban biodiversity in an era of climate change;

(3) Address areas of conflict between biodiversity conservation targets and human dimensions of climate change adaptation goals in cities in terms of the spatial patterning of land cover classes distributed across urban landscapes.

This study provides valuable data pertaining to the role of landscape patterns in affecting the overall level of biodiversity in urban landscapes in an era of climate change with particular emphasis on what researchers have observed in recent years with reference to species' behaviours in space and time (q.v. Chapter 2).

\subsubsection{Study II: A local perspective}

To adjust and confirm the results derived from Study I in a New Zealand context, Study II is undertaken using semi-structured interviews with a limited number of New Zealand subject-matter experts. In the course of the interviews, interviewees were asked to discuss and interpret the results derived from Study I, as well as address some specific local biodiversity issues in relation to spatial requirements of the selected keystone species and the local impacts of climate change (q.v. Chapter 3).

\subsubsection{Study III: Spatial analysis}

In order to be site- and species-specific, a case study of the Wellington urban landscape is conducted in Study III (q.v. Chapters 4-8). This study consists of a comprehensive and complex 
software-aided spatial analysis of landscape pattern in the study area with reference to the relationship between composition and configuration of land cover classes and the behaviours of the selected keystone species in space and time using GIS, FRAGSTATS, and a core set of landscape metrics. This study was undertaken in three levels of patch, class, and landscape (Table 1.7). The spatial hierarchy between patch, class, and landscape was taken into particular consideration in the course of the research as strongly suggested by other researchers (Forman, 1995; Botequilha Leitao et al., 2006) (cf. Table 1.8). In order to be more specific, in each chapter, the spatial composition and configuration of the current landscape pattern is quantified, measured and analysed in terms of a number of the most relevant components of landscape pattern depending on the nature and scope of the research, data availability and limitation, as well as the scale of analysis (Table 1.8). Therefore, it should not be assumed that the sequence of chapters represents the importance of analysis in each step at a higher level because Study III does not follow a linear pattern. Analyses reported in Chapters 4 to 8 should be collectively considered as integrated sets of information about the spatial composition and configuration of land cover classes in the study area. Taken together, Study III provides a comprehensive and in-depth understanding of the potentials and barriers for the conservation of indigenous biodiversity through spatial planning in the study area (q.v. Chapters 4-8).

Table 1.7

Scale of analysis and rationale behind chapters dedicated to report Study $\mathrm{III}^{31}$.

\begin{tabular}{|c|c|c|}
\hline Chapter & Scale & Rationale \\
\hline 4 & Landscape & $\begin{array}{l}\text { To provide the extent of land cover classes and a picture of the current proportion } \\
\text { of indigenous and exotic flora in the study area and address the long-term debate } \\
\text { relating to the presence/benefits of indigenous vs. exotic plant species in New } \\
\text { Zealand urban landscapes from a spatial perspective. }\end{array}$ \\
\hline 5 & Landscape & $\begin{array}{l}\text { To provide an understanding of the key role of landform in relation to land cover } \\
\text { patterns in spatial planning for urban biodiversity and reveal spatial opportunities } \\
\text { for future biodiversity conservation in the study area. }\end{array}$ \\
\hline 6 & Landscape & $\begin{array}{l}\text { To provide spatial information about the status of land cover patterns in the study } \\
\text { area before the arrival of humans and address spatial opportunities for informed } \\
\text { landscape restoration practices through natural regeneration mechanisms. }\end{array}$ \\
\hline 7 & Class & $\begin{array}{l}\text { To provide a detailed picture of the current composition and configuration of land } \\
\text { cover type classes containing different forms of plant life in relation to fauna } \\
\text { species' behaviours in space and time to examine if the current patterns are } \\
\text { capable of safeguarding indigenous fauna against the local impacts of climate } \\
\text { change in the study area. }\end{array}$ \\
\hline 8 & Patch & $\begin{array}{l}\text { To identify, rank, and prioritise the most suitable urban parklands/reserves to be } \\
\text { considered as future or expanded pest-free urban wildlife sanctuaries in the face of } \\
\text { future climate change impacts for those vulnerable fauna that cannot survive } \\
\text { outside the pest-free urban wildlife sanctuaries. }\end{array}$ \\
\hline
\end{tabular}

\footnotetext{
${ }^{31}$ At the beginning of each chapter, scientific arguments have been made to justify the chosen scale of analysis. In addition, the rationale behind each chapter has been articulated in detail to show the relevance of analysis to Study III.
} 
Table 1.8

Landscape pattern analysis in Study III in terms of the most important components of landscape pattern, namely (1) indigenousness, (2) land cover heterogeneity, (3) land surface perviousness, (4) patch size, (5) connectivity and proximity, (6) edge density, (7) landform diversity, and (8) shape complexity in three levels of analysis: Landscape (L), Class (C), and Patch (P) (cf. Table 1.7).

\begin{tabular}{|c|c|c|c|c|c|c|c|c|c|}
\hline Study III & 1 & 2 & 3 & 4 & 5 & 6 & 7 & 8 & Analysis level \\
\hline Chapter 4 & * & * & * & & & & & & $\mathrm{L}$ \\
\hline Chapter 5 & * & & & & & & * & & $\mathrm{L}$ \\
\hline Chapter 6 & * & & & & * & & & & $\mathrm{L}$ \\
\hline Chapter 7 & & & & * & * & * & & * & $\mathrm{C}$ \\
\hline Chapter 8 & * & * & * & * & * & * & * & * & $\mathrm{P}$ \\
\hline Analysis level & LCP & LP & LP & $\mathrm{CP}$ & LCP & $\mathrm{CP}$ & LP & $\mathrm{CP}$ & \\
\hline
\end{tabular}




\section{CHAPTER 2}

Study I: A questionnaire-centred survey of international researchers

\subsection{RESEARCH AIMS}

Chapter 2 reports on Study I. The aim of this study is to gather a range of data that is not currently available in the literature. In particular, Study I was conducted to:

(1) Finalise the list of the most important components of landscape patterns affecting the overall level of urban biodiversity in an era of climate change;

(2) Rank and weight the most important components of landscape patterns in terms of importance to urban biodiversity in an era of climate change;

(3) Address areas of conflict between biodiversity conservation targets and human dimensions of climate change adaptation goals in cities in terms of the spatial patterning of land cover classes distributed across urban landscapes.

\subsection{AN OVERVIEW OF INTERNATIONAL URBAN BIODIVERSITY LITERATURE}

\subsubsection{The state of the art in spatial ecology of urban fauna}

Spatial dimensions of planning for biodiversity have been well documented in urban landscapes (Müller and Kamada, 2011; Elmqvist et al, 2013; McCleery et al., 2014; Adams, 2016) because the spatial patterning of landscapes (i.e. human interventions to determine and alter the number, type, extent, size, shape, and location of land cover classes) has a widespread influence on the presence, richness, and abundance of urban fauna (q.v. Section 1.1.6; Figures 1.4 and 1.5). Recent evidence demonstrates that the survival of urban wildlife species depends, to a large extent, on the 
composition and configuration of land cover classes cumulatively characterising landscape patterns. Based upon the literature reviewed in this research (q.v. Section 1.2.3), the most important components of landscape pattern affecting biodiversity have been listed but the final confirmation of this list was made after Study I (Table 2.1), where researchers were surveyed. Information summarised on Table 2.1 provides a starting point for carrying out Study I. The overall level of urban biodiversity may be influenced by changes in landscape patterns (Table 2.2).

As stated in Chapter 1 (q.v. Section 1.1.6), fauna and humans do not necessarily require the safeguarding of the same landscape patterns against the impacts of climate change (Table 2.3). Socioeconomic factors play a major role in land cover change in human-occupied landscapes worldwide (Meyer and Turner, 1992; Lambin et al., 2001; Xiao et al., 2006; Groffman et al., 2014). Therefore, less heed is typically given to biodiversity conservation in an era of climate change in such landscapes, and accordingly more emphasis is placed on human needs and demands. Although fauna should have at least equal status because they contribute to producing vital ecosystem services upon which humans are dependent for survival (Balvanera et al., 2006; Cardinale et al., 2006; q.v. Section 1.1.2), finding a middle ground, or devising 'win-win' scenarios may be the key in urban landscapes to creating a balance between what fauna and humans require from landscape patterns in a climate that continues to change. The two following sections provide an overview of the results derived from the literature review (q.v. Section 1.2.3).

\subsubsection{Urban green space as wildlife habitats}

The survival of urban fauna including the presence, richness, and abundance of species depends, to a large extent, on the composition and configuration of land cover classes cumulatively characterising landscape patterns. Recent review studies show that changes in the composition and configuration of urban wildlife habitats (i.e. a wide range of green spaces in urban landscapes) may affect conditions upon which wildlife interact with the structure of habitats necessary to survive (Nielsen et al., 2014; Beninde et al., 2015; q.v. Table 2.1). Table 2.2 summarises key examples of studies from twenty-eight countries addressing the role of the components of landscape pattern affecting fauna in urban landscapes.

\subsubsection{Urban green space as a human adaptation tool}

The spatial composition and configuration of patches of vegetation also play an important role in facilitating or hindering human adaptation to the local impacts of climate change in urban landscapes. The ill-effects of climate change on cities and human settlements are multifaceted and 
emerge in various forms including sea level rise (Frazier et al., 2010; Hansen, 2010; Hurlimann et al., 2014; Abdrabo and Hassaan, 2015), drought (Chamaillé-Jammes et al., 2007; Logan et al, 2010; Liu and Piao, 2013; Nam et al., 2015), flooding (Veijalainen et al., 2010; Chang and Huang, 2015), wildfire (Liu et al., 2015), landslide (Andersson-Sköld et al., 2013; Middelbeek et al., 2014), heat island effects (Dugord et al., 2014; Luo and Asproudi, 2015; van Hove et al., 2015), and windstorms (Roaf et al., 2009). While these impacts affect human wellbeing, some of them can be mitigated through informed interventions in the spatial composition of configuration of patches of vegetation distributed over urban landscapes (Zhou et al., 2011; Feyisa et al., 2014; Asgarian et al., 2015; Myint et al., 2015).

\subsubsection{Areas of conflict between wildlife and humans: an overview of examples in the literature}

There are four areas of conflict between what wildlife species require and what humans expect from ecosystem services when the the central issue in urban landscapes is the spatial patterning of patches of vegetation for tackling the impacts of climate change ${ }^{32}$. These can be discussed in relation to the four components of landscape pattern (i.e. indigenousness, connectivity and proximity, edge density, and shape complexity). These areas of conflict have been classified in Table 2.2.

First, although indigenous plants are key to ensuring the continuation of biodiversity in urban landscapes (q.v. Section 4.4), some exotic species may be socio-economically more acceptable for carbon sequestration and/or storage (Ausseil et al., 2013; Ministry for Primary Industries, 2015; Setälä et al., 2016; McHale et al., 2017) ${ }^{33}$. In the context of climate change, there is evidence in the literature to show some exotic plant species may contribute to human well-being. Feyisa et al. (2014), for example, showed that in Addis Ababa, Ethiopia the cooling effect of exotic and fast-growing Eucalyptus sp., (introduced from Australia in 1894) is greater than that of other native plant species. In addition, research shows that some exotic flora may provide indigenous fauna with essential food during winter or provide suitable opportunities for initiating ecological succession towards indigenous plant communities (q.v. Chapter 4). Therefore, strict use of only indigenous plants in urban landscapes should not be considered to be a clear-cut response to biodiversity loss, because urban landscapes already contain large proportions of non-indigenous to indigenous plants, and these can be beneficial to urban ecosystems in several specific ways.

\footnotetext{
${ }^{32}$ Human-wildlife conflict has been a critical area of research for several decades (Manfredo and Dayer, 2004; Dickman, 2010). The nature of conflicts varies in space and time (Madden, 2004). However, it is not within the scope of this research to address human-urban-conflict in its broad sense.

${ }^{33}$ For example, pine is seen as desirable species because it sequesters carbon but it is not good for biodiversity, soil quality, and water health (q.v. Pedersen Zari, 2012).
} 
Second, recent evidence shows that connectivity and proximity between patches of vegetation in urban landscapes can contribute to human adaptation to climate change by reducing the negative impacts imposed by rising temperatures. For example, a study of the relationship between landscape patterns and land surface temperatures in Denver in the United States of America shows a negative correlation between land surface temperatures and connectivity and proximity between patches of vegetation (Rhee et al., 2014). A spatial analysis of land cover patterns in Isfahan in Iran demonstrates the positive role of connectivity and proximity between patches of vegetation in decreasing land surface temperatures in urban landscapes (Asgarian et al., 2015). Similarly, connectivity and proximity can facilitate species movement between habitats and this, consequently, can give rise to more sustainable species-ecosystem mutualisms in urban landscapes (q.v. Tables 2.2 and 2.3; Section 6.2.2). Such a convergence of goals can be considered a clear reason to work towards providing a sustainable network of connected patches of vegetation in urban landscapes that will protect both humans and wildlife species from the adverse effects of climate change. There is also evidence to indicate that connectivity and proximity between patches of vegetation can lead to the dispersal of weeds and pests (Sullivan et al., 2009), and evidence shows this spatial characteristic can increase the risk of widespread wildfire and the spread of diseases among species (Botequilha Leitao et al., 2006). These issues are extremely urgent in New Zealand because the country is suffering from widespread dispersal of pests and weeds (q.v. Table 1.5 and Section 8.2).

Third, while a higher rate of shape complexity and accordingly edge density may decrease the quality of wildlife habitats and consequently affect urban biodiversity, it reduces urban heat island effects through increasing the cooling effects of urban green spaces. A study of 21 urban parks in Addis Ababa, Ethiopia, for example, revealed that an increase in shape complexity and edge density could increase the distance to areas that benefitted from the park cooling effect (Feyisa et al., 2014). This example is compatible with the results of a study conducted in the city of Aksu, China (Maimaitiyiming et al., 2014), where researchers showed greater edge density resulting from higher shape complexity could effectively reduce land surface temperatures in urban landscapes without the need for increasing the total green space area. Similarly, a study of Ulsan in South Korea (Park and Cho, 2016), suggests linear patches of vegetation (i.e. belt-shaped or green corridors) are more effective in cooling their surroundings compared to compact patches of vegetation (i.e. circles or rectangles). This finding is consistent with Feyisa et al., 2014, Kong et al., 2014 and Asgarian et al., 2015. Therefore, while compact patches of vegetation may benefit wildlife species through providing more core area and less edge, elongated ones may be more beneficial for people suffering from rising temperatures. 
As humans and wildlife species coexist in urban landscapes, whether spaces (1) are made up of indigenous vs. exotic, (2) are spatially isolated or connected, (3) are spatially compact or dispersed, (4) or have a high or low edge density could remain challenging issues of conflict in landscape architecture research, particularly in the contemporary urban age, when urban policy makers are seeking solutions to adapt cities to the local impacts of climate change ${ }^{34}$ (Figure 2.1). These issues will be addressed and discussed in detail in Study I (q.v. Section 2.4.5).

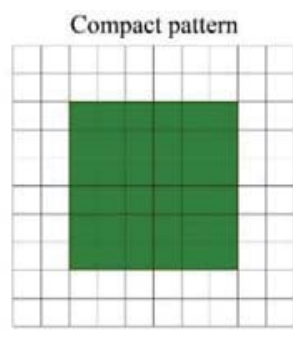

$\wedge$
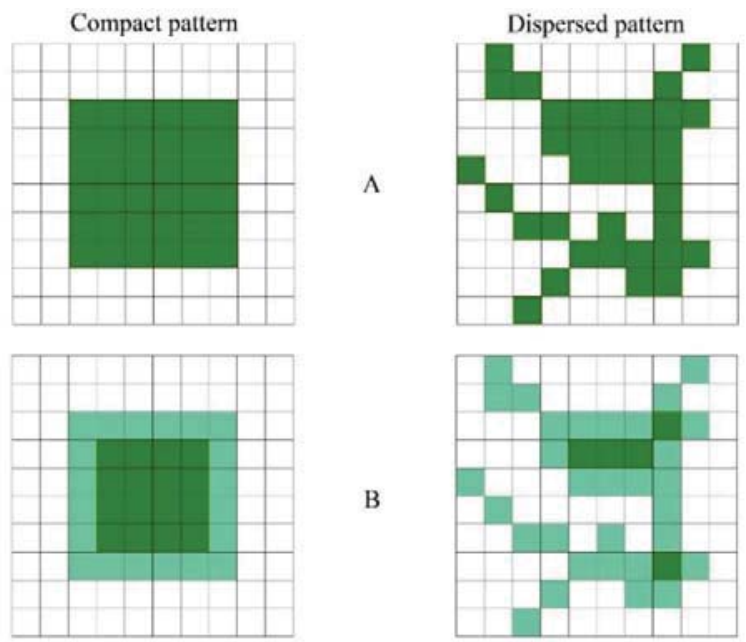

B
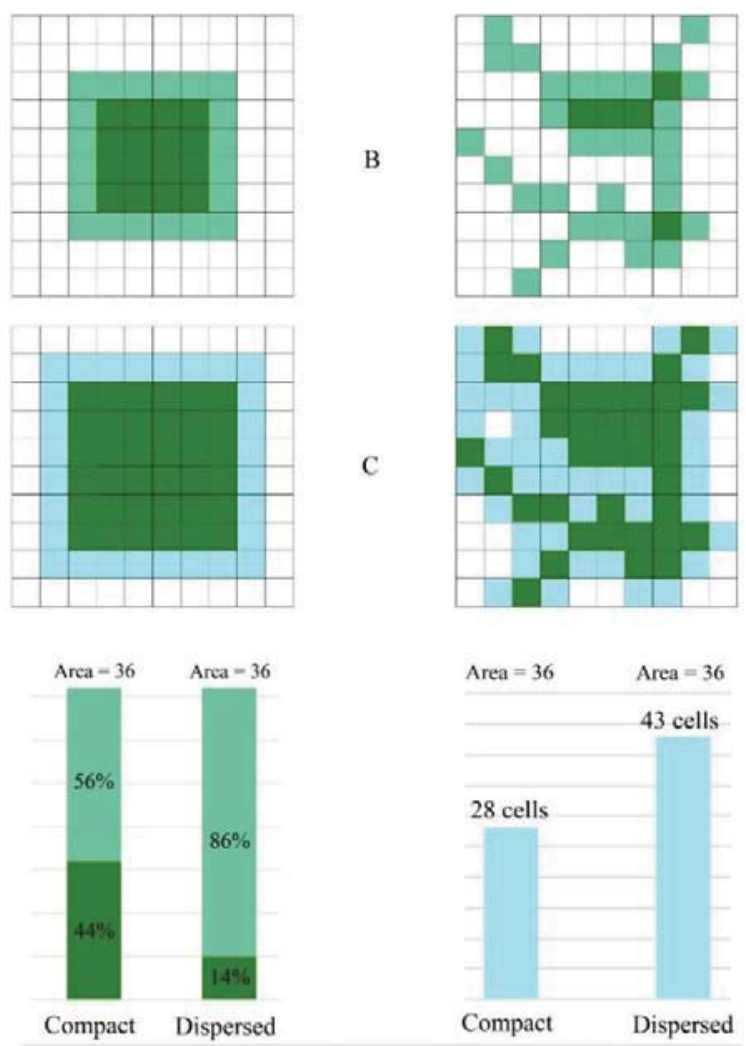

Green space Edge area

Cooling effect/distance

Figure 2.1. An example of areas of conflict between wildlife species and humans Although green space area in both cases is equal (A), shape complexity, and thereby edge density, differs profoundly. The compact pattern is suitable for protecting wildlife species from edge effects (B), whereas the dispersed pattern is more suitable for mitigating the urban heat island effect for people (C).

${ }^{34}$ Such paradoxical functioning of ecosystems in non-urban environments is, however, less likely because in natural landscapes, planning is solely focused on wildlife conservation while in urban landscapes both wildlife species and humans must be taken into consideration in the process of planning patches of vegetation. This in itself makes this process more complex and multi-dimensional. 
Table 2.1

Main components of landscape pattern composition and configuration, their definitions and rationale used in this research based upon seminal references in landscape ecology (cf. Tables 1.1, 2.2, and 2.3).

\begin{tabular}{ll}
\hline Components of landscape pattern & Definition and rationale \\
\hline Indigenousness & Definition: the state of being indigenous/native in terms of land cover type classes present in an urban context. \\
& Rationale: it is argued that overall level of biodiversity and long-term ecological integrity depends on total \\
& number of indigenous plants present at a given landscape (Andren, 1994; Forman, 1995; Fischer et al., 2009; \\
& Hahs et al, 2009; Aronson et al., 2014).
\end{tabular}

Land cover heterogeneity

Land surface perviousness

Patch size

Connectivity and proximity

Edge density and contrast

Landform diversity

Shape complexity
Definition: the diversity of different land cover type classes in patch or landscape levels.

Rationale: the number of habitat types is positively related to biodiversity across landscapes (Forman and Godron, 1986; Forman, 1995; Dramstad et al., 1996; Botequilha Leitao et al., 2006; Fischer et al., 2009).

Definition: the ability of a particular land cover type or landscape to absorb run-off caused by rainfall or sequester and store carbon dioxide in soil or vegetation.

Rationale: there is a positive relationship between land surface perviousness and bird diversity and abundance in urban landscapes (Forman, 1995; Fernández-Juricic and Jokimaki, 2001; Botequilha Leitao et al., 2006).

Definition: total area of a particular land cover type on a patch or landscape level.

Rationale: other variables being equal, an individual large habitat can support more species because it is regarded as a large pool of species genes (Mac Arthur and Wilson, 1967; Forman and Godron, 1981; Forman, 1995; Dramstad et al., 1996; Fischer et al., 2009).

Definition: the spatial distance between patches of a particular land cover type.

Rationale: spatial connectivity is believed to be vital to biodiversity facilitating the process of gene flow between patches of vegetation (Shaffer, 1981; Naveh, 1994; Forman, 1995; Bennett, 1999; Honnay et al., 2003; Fischer et al., 2009).

Definition - edge density: perimeter of a patch of particular land cover type exposed to other land cover types. Rationale: as edge density increases, the patch interaction with its surroundings increases either positively or negatively (Andren, 1994; Murica, 1995; Dramstad et al., 1996).

Definition - edge contrast: dissimilarity between adjacent land cover types.

Rationale: while high edge contrast may be regarded as a barrier in front of disease spread, pest and weed dispersal, and wildfire, low edge contrast may be considered as a strategy for facilitating species' movement between patches of vegetation (Forman, 1995; Dramstad et al., 1996; Botequilha Leitao et al., 2006).

Definition: diversity of elevations, slopes, and aspects.

Rationale: it is argued that patches with diverse topography provide a spectrum of variable climatic conditions for a wide range of species and this in itself supports biodiversity in a changing climate (Rorison et al., 1986; Markham et al, 1993; Pernetta et al, 1994; Halpin, 1997; Noss, 2001; Fischer et al., 2009; Reside et al., 2014; Dobrowski et al., 2011; Olson et al., 2012).

Definition: the degree to which a wildlife habitat is dissimilar to circle-shaped pattern.

Rationale: the proportion between edge-core areas in a given patch depends on patch shape affecting biodiversity (O’Neill et al., 1988; Noss and Cooperrider, 1994; Dramstad et al., 1996; Botequilha Leitao et al., 2006). 


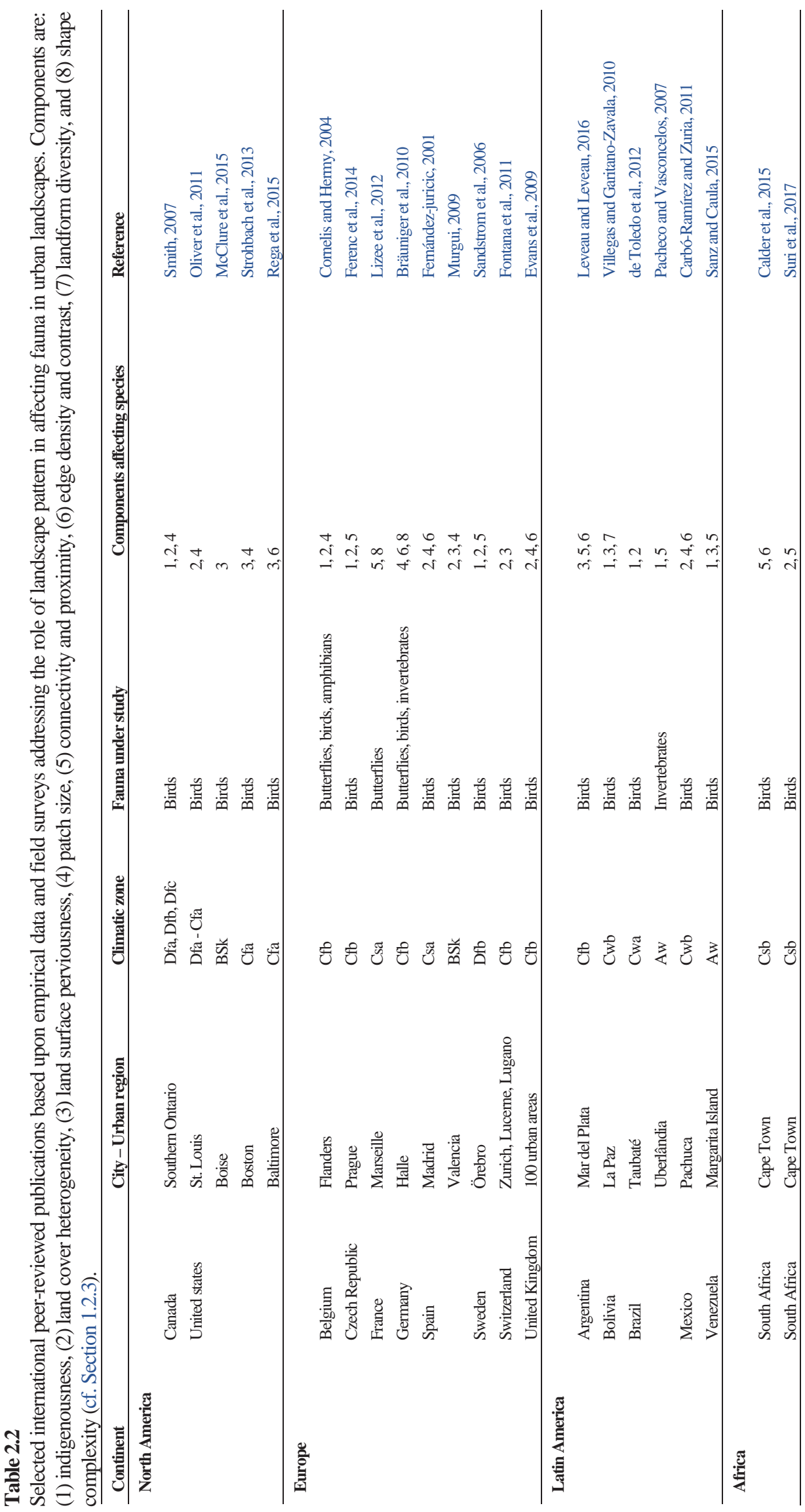




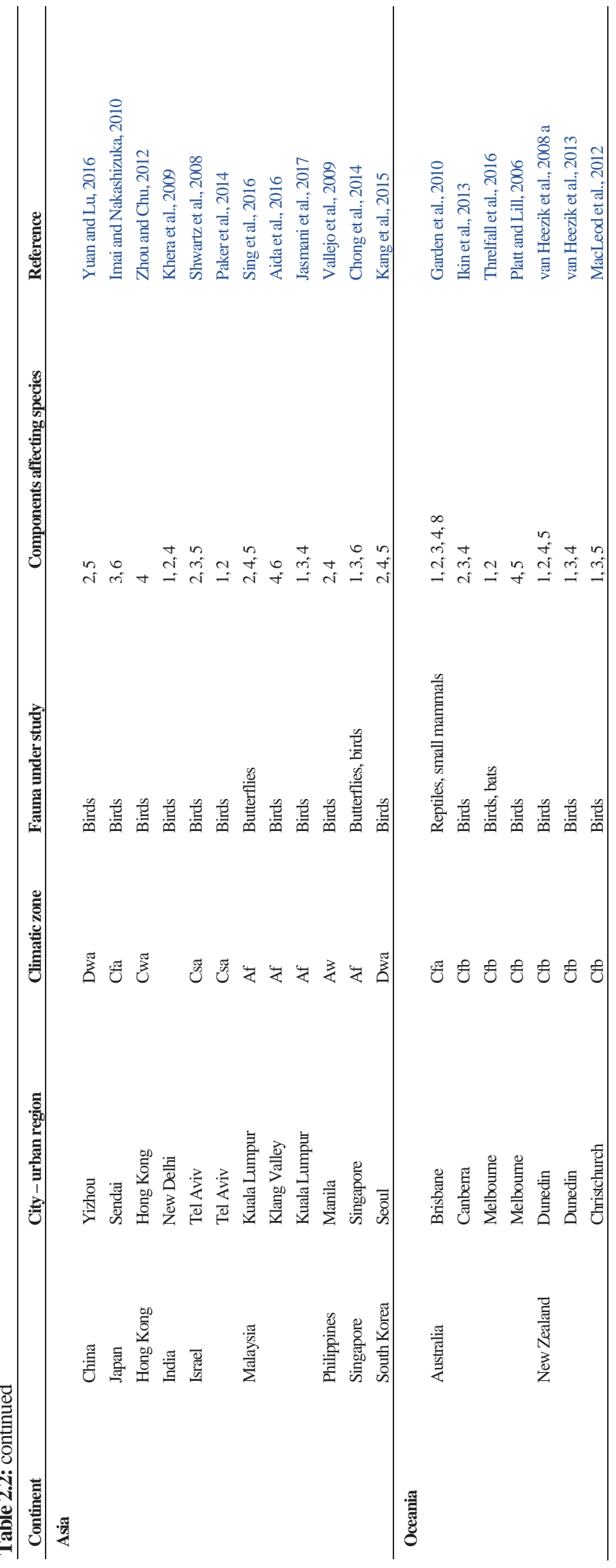




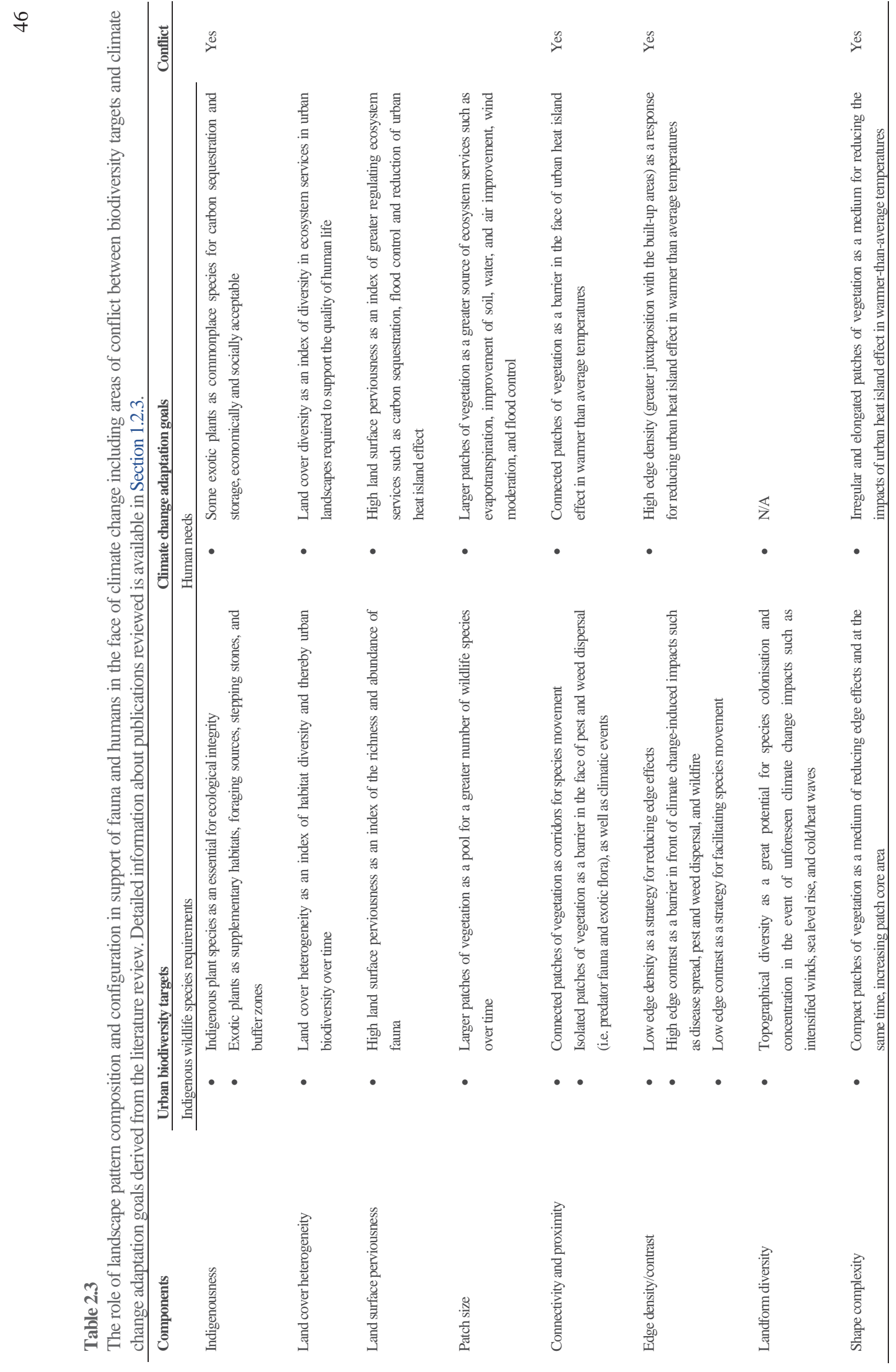




\subsection{MATERIALS AND METHODS}

\subsubsection{Questionnaire design and sampling process}

With reference to the research aims in Study I, a questionnaire was designed (q.v. Appendix I). To design the questionnaire, best practice research standards were carefully observed (Oppenheim, 2000; Groat and Wang, 2002; Deming and Swaffield, 2011; Rea and Parker, 2014; Bryman, 2015).

The questionnaire was designed using an online survey tool called Qualtrics in order to facilitate the effective circulation of the questionnaire. The questionnaire included five general questions designed to address the participants' characteristics, a scoring question designed using a five point Likert Scale ${ }^{35}$ to score the eight components of landscape pattern affecting the presence, richness, and abundance of fauna (q.v. Tables 2.1 and 2.2) ${ }^{36}$, and four open-ended questions to address and discuss areas of conflict between what fauna require and what humans need in terms of spatial patterning of landscapes in the face of climate change impacts in urban landscapes.

A 'filter question' (Oppenheim, 2000) was included at the beginning of the questionnaire to ensure that potential participants had already undertaken empirical research on the connection between landscape pattern composition and configuration and urban fauna in recent years.

Two techniques were applied to find relevant specialists to survey: first, a comprehensive search was conducted on scientific databases using a wide range of keywords to find relevant publications and accordingly the email contact address of authors (i.e. potential participants) based upon their documented empirical research-based papers published in journals of international repute; second, a snowball sampling approach (Goodman, 1961; Biernacki and Waldorf, 1981; Noy, 2008) was followed to increase the odds of participation and reduce the possibility of missing potential participants worldwide. This means that at the end of the questionnaire, participants were asked to forward the link of the questionnaire to researchers who might be able to contribute to this research. In the process of sampling, three major questions were taken into consideration to find the people around the world most relevant to the research:

(1) Does the researcher have enough relevant experience to contribute to the research based on publications in English language journals of international repute? If so;

\footnotetext{
${ }^{35}$ A five-point Likert scale was used, as recommended by Deming and Swaffield (2011) for survey studies in landscape architecture research. This spectrum is also widely utilised in the process of multi-criteria analysis, where ranking and weighting are necessary (q.v. Section 8.3.1).

${ }^{36}$ Participants were invited to add more items to the list (q.v. Section 2.4.4).
} 
(2) Has the researcher already undertaken empirical research on the spatial ecology of wildlife species in urban landscapes? If so;

(3) Did the sampling process take the geographical diversity of samples into consideration?

The questionnaire was designed so that practical information about how to foster the overall level of biodiversity among fauna in urban landscapes could be derived from it. Therefore, results do not differ between taxonomic groups, but useful information was deduced about the spatial design of patches of vegetation as a whole, in a way that contributes to the presence, richness, and abundance of fauna in cities. The results are more applicable to avifauna conservation and, to a lesser extent, small mammals, and reptiles because the coexistence of these groups with humans in urban areas is often commonplace. Considering other groups of fauna including insects and large mammals was not within the scope of this research ${ }^{37}$. Participants were asked to take the role of climate change into account while answering the questions. This was achieved by providing an information sheet containing key information about the relationship between climate change and biodiversity loss presented at the start of the online questionnaire. Thus, the responses inherently included climate change as an influential factor. Potential participants had the right to choose not to answer any question, withdraw from the study before 31 March 2017, ask any questions about the study at any time and be able to read any reports of this research by emailing the researcher to request a copy.

\subsubsection{Sample size}

To determine the sample size, the "saturation point” concept was applied (Flick, 1998; Seale; 1999; Guest et al, 2006; Bowen; 2008). Saturation point in a questionnaire-centred survey can be found when and where no new data is returned from the participants. The sample size may be affected by many factors including the total population of the targeted group (Israel, 1992).

\footnotetext{
${ }^{37}$ There are at least four reasons for this exclusion. First, the presence of large mammals in urban landscapes is not common, specifically in New Zealand where the native fauna is almost exclusively avian. Second, the home range size of large mammals is often studied at the regional scale while the scale of this research is urban. Third, insect diversity and biology is thought to be a unique field of study (Stork, 1988; Smith et al., 2015). Fourth, this research is conducted at the broad scale (i.e. urban) whereas addressing the habitat requirements of insects requires studying biodiversity at the micro scale (i.e. very small patches of vegetation).
} 
In urban landscapes, this is a relatively new branch of research. Therefore, only a small fraction of scholars could participate in this survey because only a limited number of researchers in the fields of landscape architecture, urban planning, wildlife management, zoology, biology, and ecology are profoundly familiar with the topic, and have a track record of research on the spatial ecology of urban fauna with particular emphasis on avifauna, small mammals and reptiles. In comparison with similar studies in recent years, such as Meijering et al. (2015) ( $\mathrm{n}=86)$, Martin et al. (2005) ( $n=20)$, and Sternberg, et al. (2015) $(n=45)$, the number of researchers that participated in this survey was large and was deemed to be sufficient $(n=87)$. The survey was conducted anonymously and the collected data was aggregated in order to increase the level of individual privacy and remove any sign or information that might reveal the identity of participants.

\subsubsection{Distribution of the questionnaire}

The survey content was approved by the Victoria University of Wellington Human Ethics Committee on the $18^{\text {th }}$ of October 2016 (Appendix II). The online questionnaire was circulated via email over three months, from 25 October 2016 to 24 January 2017, to a group of appropriate researchers and scientists worldwide. A reminder email was also sent to each potential participant two to four weeks after sending the first invitation email to encourage him/her to take the survey.

The survey was conducted anonymously, and the collected data was aggregated in order to increase the level of individual privacy and remove any sign or information that might reveal the identity of participants.

Ultimately, 87 useable questionnaires were collected, demonstrating perhaps the highest rate of participation to date in a worldwide survey on this specialised area of landscape architecture research.

\subsubsection{Geographical distribution of participants}

Participants were classified into three main groups based upon the countries in which they had conducted empirical research, namely (1) Group A (all participants worldwide), (2) Group B (participants whose research experience includes undertaking empirical research in at least one country in the Southern Hemisphere), and (3) Group C (participants whose research experience includes undertaking empirical research in at least one country in Oceania). Groups B and C are the subsets of Groups A and B, respectively. Participants were grouped in this way to study how and to 
what degree the opinions of participants in Groups B and C may respectively influence those of Groups A and B.

\subsubsection{Statistical analysis}

In the first section of the survey, the eight most important components of landscape pattern affecting fauna in urban landscapes were sorted and participants were asked to score them, using a score from 1 the least important, to 5 the most important, in terms of each component's unique influence on urban fauna in an era of climate change. The total score was then calculated and weighted based on the Likert Scale. Finally, the weighted scores were normalised. The repeated measures ANOVA were also performed to examine (for each group) if mean responses differ for at least one component of landscape pattern.

Different statistical tests including Mauchley's test of sphericity and the Greenhouse Geisser correction were also carried out to reveal differences within and between groups using SPSS v.24 (Field, 2013). Pairwise comparisons with the Bonferroni correction applied were run to compare the importance of each component of landscape pattern to others within each group. The analysed data was tabulated to provide a basis for quantitative comparisons.

In the second section of the survey, four areas of conflict between fauna and humans in urban landscapes (q.v. Table 2.3) were addressed using four open-ended questions. Participants were able to answer the questions and share their experiences in a descriptive manner. These questions provided a basis for a qualitative analysis of responses (Well et al., 1997; Hsieh and Shannon, 2005).

\subsection{RESULTS}

\subsubsection{Aggregated information on participants' characteristics}

Over three months, 87 useable online questionnaires were collected. Participants from 69 universities and research centres with experience in conducting empirical research on the topic under investigation in 39 different countries submitted their responses. Some of the participants used phrases such as various countries in Africa, Europe, Central Europe, and approximately n countries, instead of specifying the individual countries in which they conducted their research. Thus, it seems that the geographical distribution of the survey is likely to be beyond what has been documented in this study (Table 2.4). 
According to the collected questionnaires, $28.74 \%$ of participants $(n=25)$ have conducted empirical research on spatial dimensions of landscape planning for urban biodiversity in more than one country. Some of the participants mentioned that they have undertaken long-term empirical research in urban sites ranging from 10 to 15 years. In response to the question on the number of projects undertaken on this topic since 2000, 94.25\% of the participants $(n=82)$ answered the question affirmatively. Overall, the survey reflects collective wisdom stemming from at least 325 research projects worldwide providing a robust dataset of informed responses. That is equivalent to an average of 3.74 projects per participant since 2000.

According to the responses recorded from $90.80 \%$ of the questionnaires $(n=79)$, the research projects were conducted in various scales ranging from very small patches of vegetation (e.g. house backyards) to relatively large landscapes (e.g. metropolitan regions), and relate mostly to avifauna, and to a lesser extent, small mammals and reptiles. This, in turn, ensures a comprehensive knowledge of a wide spectrum of different ecosystems at the urban landscape scale and builds up a deep understanding of ways through which biodiversity, as a whole, can be effectively partially safeguarded as the climate changes. 
Table 2.4

Geographical distribution of participants based upon countries in which they have conducted empirical research on the connection between landscape pattern and the presence, richness, and abundance of urban fauna.

\begin{tabular}{|c|c|c|}
\hline Region (\# of countries) & Country (\# of participants mentioned the country as a research site) & Hemisphere \\
\hline \multirow[t]{2}{*}{ North America (2) } & Canada (8) & Northern \\
\hline & United States of America (26) & Northern \\
\hline \multirow[t]{15}{*}{ Europe (15) } & Albania (1) & Northern \\
\hline & Austria (2) & Northern \\
\hline & Belgium (1) & Northern \\
\hline & Denmark (1) & Northern \\
\hline & Finland (1) & Northern \\
\hline & France (3) & Northem \\
\hline & Germany (9) & Northern \\
\hline & Italy (3) & Northern \\
\hline & Poland (1) & Northern \\
\hline & Portugal (1) & Northern \\
\hline & Slovenia (1) & Northern \\
\hline & Spain (2) & Northern \\
\hline & Switzerland (5) & Northern \\
\hline & Turkey (3) & Northern \\
\hline & United Kingdom (6) & Northern \\
\hline \multirow[t]{9}{*}{ Latin America (9) } & Argentina (1) & Southem \\
\hline & Bolivia (1) & Southem \\
\hline & Brazil (1) & Southern \\
\hline & Costa Rica (1) & Northem \\
\hline & Dominican Republic (1) & Northem \\
\hline & Mexico (2) & Northern \\
\hline & Peru (1) & Southem \\
\hline & Puerto Rico (1) & Northern \\
\hline & Uruguay (1) & Southem \\
\hline \multirow[t]{8}{*}{ Asia (8) } & China (2) & Northem \\
\hline & India (1) & Northern \\
\hline & $\operatorname{Iran}(1)$ & Northern \\
\hline & Japan (1) & Northern \\
\hline & Malaysia (1) & Northem \\
\hline & Nepal (1) & Northern \\
\hline & Republic of Korea (1) & Northern \\
\hline & Taiwan (1) & Northern \\
\hline \multirow[t]{2}{*}{ Africa (2) } & South Africa (2) & Southem \\
\hline & Uganda (1) & Northern \\
\hline \multirow[t]{3}{*}{ Oceania (3) } & Australia (9) & Southem \\
\hline & New Caledonia (1) & Southem \\
\hline & New Zealand (9) & Southern \\
\hline
\end{tabular}

\subsubsection{An expert-driven ranking model}

The eight most important components of landscape pattern affecting fauna in urban landscapes (q.v. Tables 2.1 and 2.2) were sorted and participants were asked to score them, using a score from 1 the least important to 5 the most important, in terms of each component's unique 
influence on urban fauna in an era of climate change. The total score was then calculated and weighted based on the Likert Scale. Finally, the weighted scores were normalised. All participants $(100 \%, n=87)$ answered this question; however some (very few) did not dedicate scores to some components of landscape pattern. Different statistical tests were carried out to reveal differences within and between groups. The analysed data was tabulated to provide a basis for comparison (Tables 2.5 to 2.10).

\subsubsection{Ranking in a global context - Group A}

On a global scale, responses of all participants $(n=87)$ were taken into consideration (q.v. Table 2.4). The results show that patch size, connectivity and proximity, and land cover heterogeneity were ranked by Group A as the most important components of landscape pattern, weighted 15.6\%, $15.2 \%$, and $14.02 \%$, respectively. After these, land surface perviousness with $12.23 \%$, landform diversity with $11.49 \%$, and indigenousness with $11.36 \%$ stood in the fourth to sixth places in terms of importance to urban biodiversity. Seventh and eighth places were dedicated to edge density and contrast and shape complexity respectively, with $10.62 \%$ and $9.48 \%$ of importance (q.v. Table 2.5; Figure 2.3).

\subsubsection{Ranking in a Southern Hemisphere context - Group B}

More than one-fourth of the participants $(25.28 \%, \mathrm{n}=22)$ classified in Group B stated that they have conducted empirical research on the connection between urban fauna and the spatial patterning of landscapes in Southern Hemisphere countries. Studies consisted of five countries in Latin America (Argentina, Bolivia, Brazil, Peru, and Uruguay), one country in Africa (South Africa), and three countries in Oceania (Australia, New Caledonia, and New Zealand) (q.v. Table 2.4). All of these countries are identified as regions of biodiversity importance (Myers et al., 2000; Olson et al., 2001). According to the results, patch size (16.52\%), connectivity and proximity (15.43\%) and land cover heterogeneity (14.88\%) were the most important components of landscape pattern affecting fauna in the urban Southern Hemisphere for this group of experts.

Dissimilar to the results from Group A, indigenousness (13.07\%) was the fourth most important component of landscape pattern in this region of the world. Land surface perviousness (11.25\%) and landform diversity (11.25\%) also stand equally in the fifth place. Finally, edge density and contrast (8.89\%) and shape complexity (8.71\%) were ranked in the next places (q.v. Table 2.5; Figure 2.2; cf. Figure 2.3). 


\subsubsection{Ranking in an Oceania context - Group C}

About one-fifth of the participants $(19.54 \%, \mathrm{n}=17)$ classified in Group C stated that they have had at least one research experience on the connection between fauna and spatial patterning of landscapes in Oceania. Studies looked at Australia, New Caledonia, and New Zealand (q.v. Table 2.4). According to Myers et al. (2000) and Olson et al. (2001), New Caledonia and New Zealand are amongst very few countries that have their entire landmass recognised as one of the world's biodiversity hotspots. Similar to Groups A and B, patch size (16.39\%), connectivity and proximity (14.49\%) and land cover heterogeneity (14.49\%) were ranked by participants as the most important components of landscape pattern. Afterwards, indigenousness (13.77\%) stood in the next place and was consistent with the results derived from Group B. Land surface perviousness (11.88\%) and landform diversity (11.88\%) were also equally ranked as the fifth most important component of landscape pattern. Edge density and contrast (8.55\%) and shape complexity (8.55\%) concurrently were identified as the least important factors affecting fauna in urban Australia, New Caledonia, and New Zealand (q.v. Table 2.5; Figure 2.3).

\subsubsection{Differences within and between groups}

Mean responses for eight components of landscape pattern in Groups A, B, and C reveal differences within groups (Figure 2.2; cf. Table 2.6). Mauchley’s test of sphericity determined that for Group A the assumption of sphericity is not met, $\mathrm{X}^{2}(27)=51.888, \mathrm{p}=0.003(\mathrm{p}<0.05)$. The Greenhouse Geisser correction was therefore applied and determined that there is a significant difference in the importance of at least one landscape pattern in Group A, $F(5.55,338.60)=29.170$, $p$ $<0.001$. The assumption of sphericity is met for both Groups B and C (both p > 0.05) (Table 2.7).

Highest similarities were observed between Groups B and C while the lowest similarities were recorded between Groups A and C. This is in agreement with differences between groups in terms of normalised weights (cf. Table 2.5). Differences in mean responses for indigenousness, patch size, and land surface perviousness are very low between Groups B and C. The highest difference between these two groups was observed in connectivity and proximity. The lowest degree of difference among Groups A, B, and C was observed in patch size. Pairwise comparisons with the Bonferroni correction were made using estimated marginal means (which differ from means reported in Tables 2.5), testing at the $5 \%$ significance level.

Looking at standard deviations calculated for each component of landscape pattern in all groups (q.v. Table 2.5), the most homogenised responses belong to Group B, while there can be seen 
more variations in responses collected from Group C and Group A. In terms of components of landscape pattern, the lowest variation in responses appertains to patch size. Next, edge density and contrast, connectivity and proximity, indigenousness, shape complexity, landform diversity, land cover heterogeneity, and land surface perviousness were ranked with regard to the degree to which the responses in all groups are homogenised (q.v. Tables 2.5). Although there are slight differences among mean responses of the first three most important components of landscape pattern in all groups, differences are not statistically significant $(\mathrm{p}>0.05)$ (Table 2.8). 
in

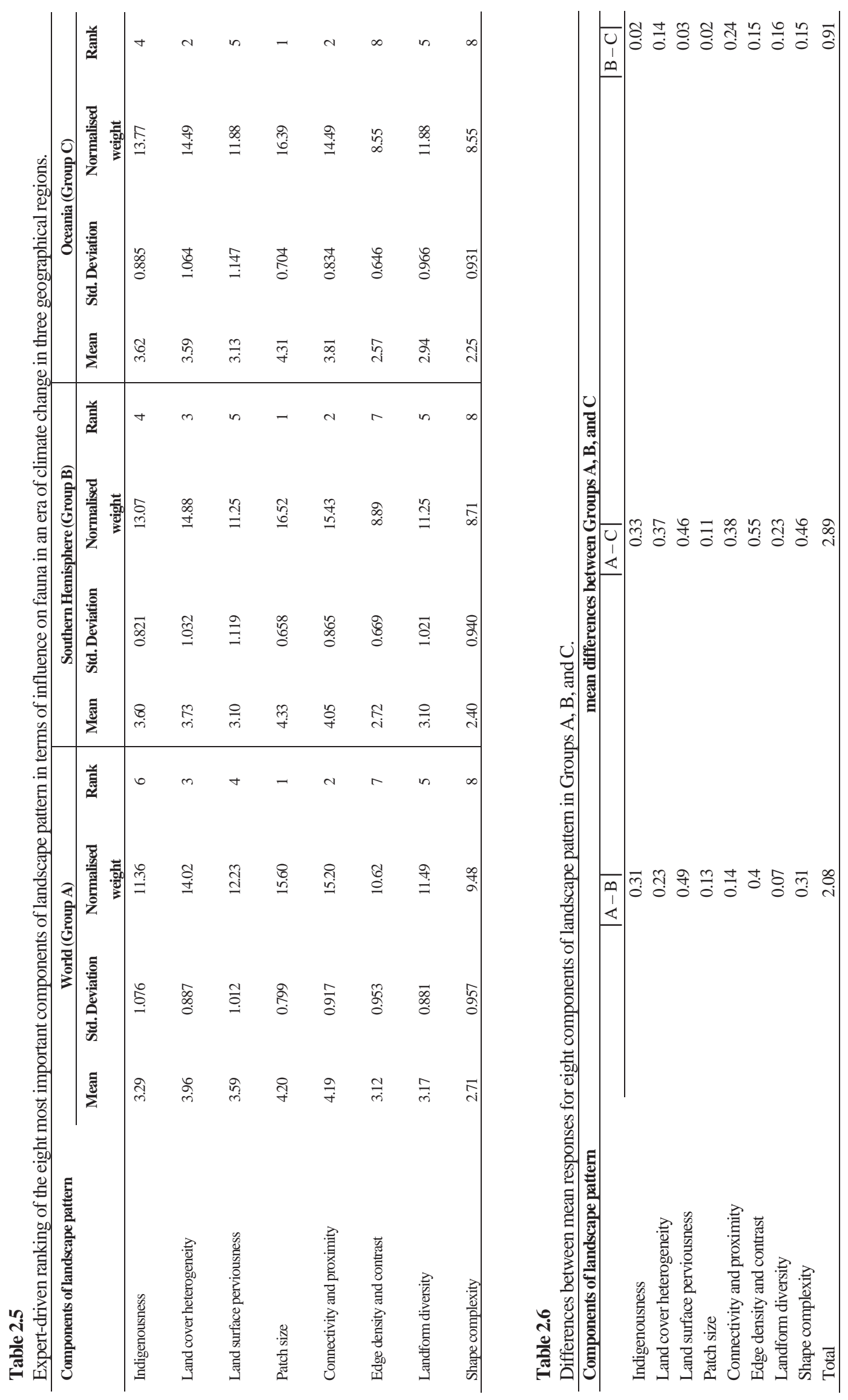


Table 2.7

Mauchly’s test of Sphericity performed for Groups A, B, and C.

\begin{tabular}{lrrrr} 
Groups & Approx. Chi-Square & df & Sig. & Greenhouse Geisser \\
\hline A & 51.888 & 27 & 0.003 & 0.793 \\
B & 36.904 & 27 & 0.122 & 0.531 \\
C & 36.838 & 27 & 0.139 & 0.494 \\
\hline
\end{tabular}

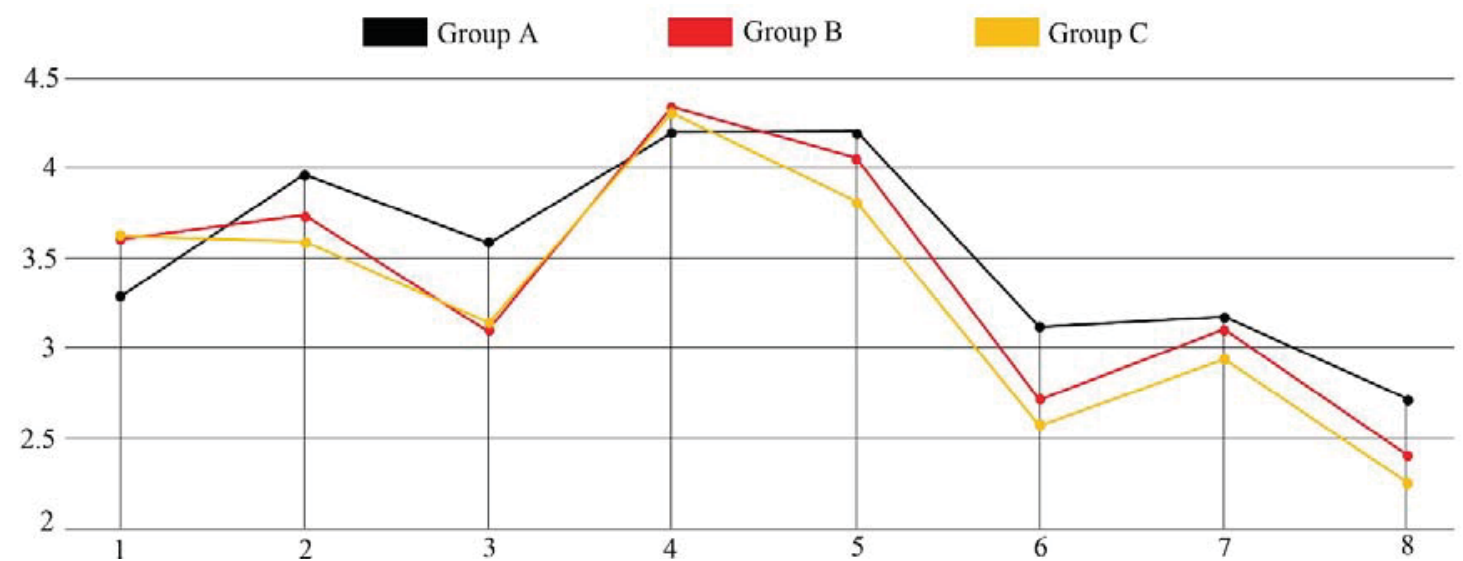

Figure 2.2. Mean responses for the eight components of landscape pattern in Groups A, B, and C. Indigenousness (1), Land cover heterogeneity (2), Land surface perviousness (3), Patch size (4), Connectivity and proximity (5), Edge density and contrast (6), Landform diversity (7), and Shape complexity (8).

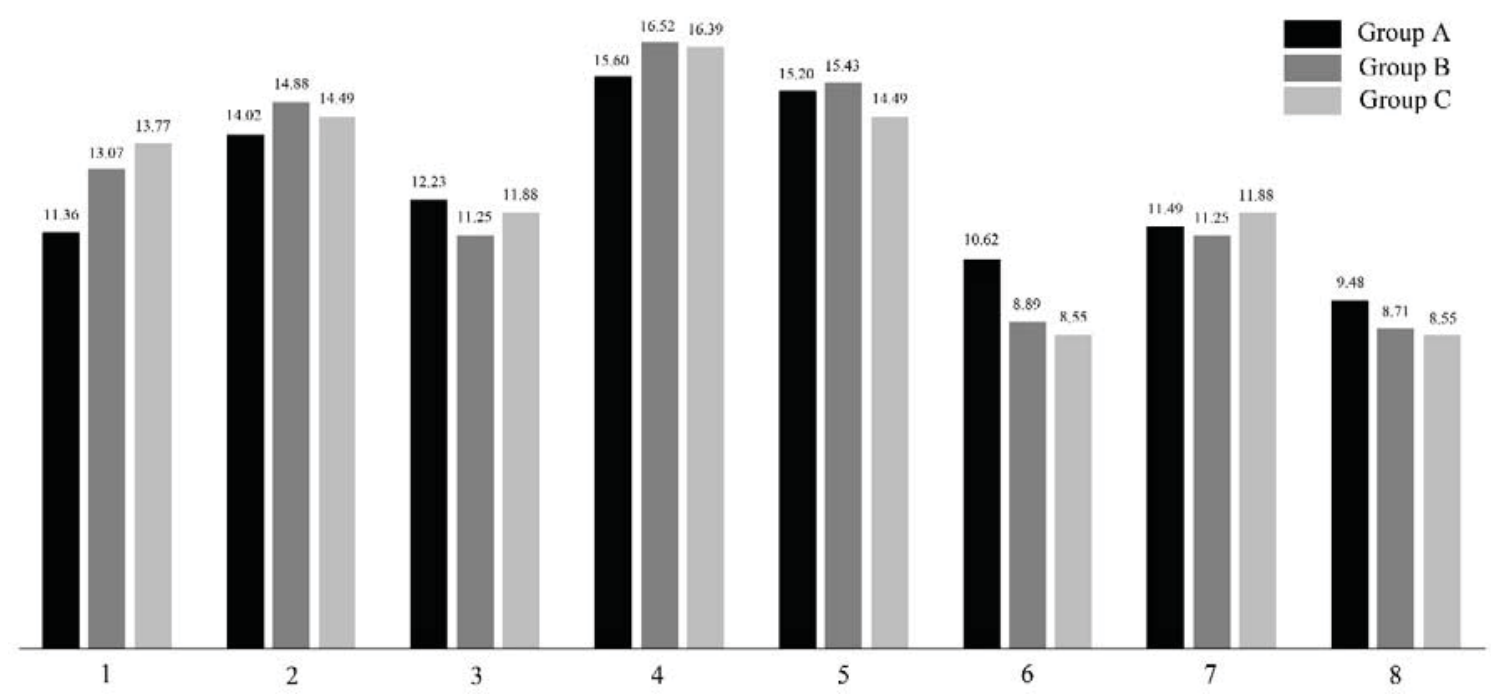

Figure 2.3. Comparison of normalised weights assigned to each component of landscape pattern by scientists and researchers in three different groups of World, Southern Hemisphere, and Oceania: (1) Indigenousness, (2) Land cover heterogeneity, (3) Land surface perviousness; (4) Patch size, (5) Connectivity and proximity, (6) Edge density and contrast, (7) Landform diversity, and (8) Shape complexity. 
Table 2.8

Pairwise comparisons made based upon estimated marginal means for Groups A, B, and C. Indigenousness (1), Land cover heterogeneity (2), Land surface perviousness (3), Patch size (4), Connectivity and proximity (5), Edge density and contrast (6), Landform diversity (7), and Shape complexity (8).

\begin{tabular}{|c|c|c|c|c|c|c|c|c|c|c|}
\hline \multirow{2}{*}{$\begin{array}{l}\text { Pairwise } \\
\text { Comparisons }\end{array}$} & & \multicolumn{3}{|c|}{ Group A } & \multicolumn{3}{|c|}{ Group B } & \multicolumn{3}{|c|}{ Group C } \\
\hline & & Mean dif. & Std. error & Sig. & Mean dif. & Std. error & Sig. & Mean dif. & Std. error & Sig. \\
\hline \multirow[t]{7}{*}{1} & 2 & $-.790^{*}$ & 0.163 & 0.000 & -0.615 & 0.401 & 1.000 & -0.455 & 0.455 & 1.000 \\
\hline & 3 & -0.387 & 0.163 & 0.584 & 0.000 & 0.253 & 1.000 & 0.091 & 0.285 & 1.000 \\
\hline & 4 & $-.952^{*}$ & 0.133 & 0.000 & -0.769 & 0.231 & 0.167 & -0.727 & 0.273 & 0.662 \\
\hline & 5 & $-1.016^{*}$ & 0.170 & 0.000 & -0.308 & 0.263 & 1.000 & -0.091 & 0.251 & 1.000 \\
\hline & 6 & 0.194 & 0.184 & 1.000 & 0.923 & 0.288 & 0.211 & 1.000 & 0.330 & 0.356 \\
\hline & 7 & 0.081 & 0.168 & 1.000 & 0.308 & 0.398 & 1.000 & 0.455 & 0.413 & 1.000 \\
\hline & 8 & 0.516 & 0.184 & 0.186 & 1.077 & 0.348 & 0.261 & 1.273 & 0.384 & 0.218 \\
\hline \multirow[t]{7}{*}{2} & 1 & $.790^{*}$ & 0.163 & 0.000 & 0.615 & 0.401 & 1.000 & 0.455 & 0.455 & 1.000 \\
\hline & 3 & 0.403 & 0.148 & 0.236 & 0.615 & 0.350 & 1.000 & 0.545 & 0.413 & 1.000 \\
\hline & 4 & -0.161 & 0.142 & 1.000 & -0.154 & 0.337 & 1.000 & -0.273 & 0.384 & 1.000 \\
\hline & 5 & -0.226 & 0.139 & 1.000 & 0.308 & 0.308 & 1.000 & 0.364 & 0.364 & 1.000 \\
\hline & 6 & $.984^{*}$ & 0.155 & 0.000 & $1.538^{*}$ & 0.183 & 0.000 & $1.455^{*}$ & 0.207 & 0.001 \\
\hline & 7 & $.871^{*}$ & 0.123 & 0.000 & 0.923 & 0.329 & 0.447 & 0.909 & 0.368 & 0.928 \\
\hline & 8 & $1.306^{*}$ & 0.137 & 0.000 & $1.692^{*}$ & 0.286 & 0.002 & $1.727^{*}$ & 0.333 & 0.011 \\
\hline \multirow[t]{7}{*}{3} & 1 & 0.387 & 0.163 & 0.584 & 0.000 & 0.253 & 1.000 & -0.091 & 0.285 & 1.000 \\
\hline & 2 & -0.403 & 0.148 & 0.236 & -0.615 & 0.350 & 1.000 & -0.545 & 0.413 & 1.000 \\
\hline & 4 & $-.565^{*}$ & 0.150 & 0.011 & -0.769 & 0.257 & 0.314 & -0.818 & 0.296 & 0.559 \\
\hline & 5 & $-.629^{*}$ & 0.161 & 0.007 & -0.308 & 0.175 & 1.000 & -0.182 & 0.182 & 1.000 \\
\hline & 6 & $.581^{*}$ & 0.163 & 0.021 & 0.923 & 0.239 & 0.064 & 0.909 & 0.285 & 0.268 \\
\hline & 7 & 0.468 & 0.159 & 0.128 & 0.308 & 0.444 & 1.000 & 0.364 & 0.509 & 1.000 \\
\hline & 8 & $.903^{*}$ & 0.154 & 0.000 & 1.077 & 0.309 & 0.127 & 1.182 & 0.352 & 0.204 \\
\hline \multirow[t]{7}{*}{4} & 1 & $.952^{*}$ & 0.133 & 0.000 & 0.769 & 0.231 & 0.167 & 0.727 & 0.273 & 0.662 \\
\hline & 2 & 0.161 & 0.142 & 1.000 & 0.154 & 0.337 & 1.000 & 0.273 & 0.384 & 1.000 \\
\hline & 3 & $.565^{*}$ & 0.150 & 0.011 & 0.769 & 0.257 & 0.314 & 0.818 & 0.296 & 0.559 \\
\hline & 5 & -0.065 & 0.126 & 1.000 & 0.462 & 0.215 & 1.000 & 0.636 & 0.203 & 0.299 \\
\hline & 6 & $1.145^{*}$ & 0.147 & 0.000 & $1.692^{*}$ & 0.208 & 0.000 & $1.727^{*}$ & 0.237 & 0.001 \\
\hline & 7 & $1.032^{*}$ & 0.158 & 0.000 & 1.077 & 0.383 & 0.441 & 1.182 & 0.400 & 0.406 \\
\hline & 8 & $1.468^{*}$ & 0.169 & 0.000 & $1.846^{*}$ & 0.296 & 0.001 & $2.000^{*}$ & 0.330 & 0.003 \\
\hline \multirow[t]{7}{*}{5} & 1 & $1.016^{*}$ & 0.170 & 0.000 & 0.308 & 0.263 & 1.000 & 0.091 & 0.251 & 1.000 \\
\hline & 2 & 0.226 & 0.139 & 1.000 & -0.308 & 0.308 & 1.000 & -0.364 & 0.364 & 1.000 \\
\hline & 3 & $.629^{*}$ & 0.161 & 0.007 & 0.308 & 0.175 & 1.000 & 0.182 & 0.182 & 1.000 \\
\hline & 4 & 0.065 & 0.126 & 1.000 & -0.462 & 0.215 & 1.000 & -0.636 & 0.203 & 0.299 \\
\hline & 6 & $1.210^{*}$ & 0.128 & 0.000 & $1.231^{*}$ & 0.201 & 0.001 & $1.091^{*}$ & 0.211 & 0.012 \\
\hline & 7 & $1.097^{*}$ & 0.147 & 0.000 & 0.615 & 0.350 & 1.000 & 0.545 & 0.390 & 1.000 \\
\hline & 8 & $1.532^{*}$ & 0.134 & 0.000 & $1.385^{*}$ & 0.241 & 0.003 & $1.364^{*}$ & 0.279 & 0.018 \\
\hline \multirow[t]{7}{*}{6} & 1 & -0.194 & 0.184 & 1.000 & -0.923 & 0.288 & 0.211 & -1.000 & 0.330 & 0.356 \\
\hline & 2 & $-.984^{*}$ & 0.155 & 0.000 & $-1.538^{*}$ & 0.183 & 0.000 & $-1.455^{*}$ & 0.207 & 0.001 \\
\hline & 3 & $-.581^{*}$ & 0.163 & 0.021 & -0.923 & 0.239 & 0.064 & -0.909 & 0.285 & 0.268 \\
\hline & 4 & $-1.145^{*}$ & 0.147 & 0.000 & $-1.692^{*}$ & 0.208 & 0.000 & $-1.727^{*}$ & 0.237 & 0.001 \\
\hline & 5 & $-1.210^{*}$ & 0.128 & 0.000 & $-1.231^{*}$ & 0.201 & 0.001 & $-1.091^{*}$ & 0.211 & 0.012 \\
\hline & 7 & -0.113 & 0.142 & 1.000 & -0.615 & 0.311 & 1.000 & -0.545 & 0.340 & 1.000 \\
\hline & 8 & 0.323 & 0.128 & 0.393 & 0.154 & 0.191 & 1.000 & 0.273 & 0.195 & 1.000 \\
\hline \multirow[t]{7}{*}{7} & 1 & -0.081 & 0.168 & 1.000 & -0.308 & 0.398 & 1.000 & -0.455 & 0.413 & 1.000 \\
\hline & 2 & $-.871^{*}$ & 0.123 & 0.000 & -0.923 & 0.329 & 0.447 & -0.909 & 0.368 & 0.928 \\
\hline & 3 & -0.468 & 0.159 & 0.128 & -0.308 & 0.444 & 1.000 & -0.364 & 0.509 & 1.000 \\
\hline & 4 & $-1.032^{*}$ & 0.158 & 0.000 & -1.077 & 0.383 & 0.441 & -1.182 & 0.400 & 0.406 \\
\hline & 5 & $-1.097^{*}$ & 0.147 & 0.000 & -0.615 & 0.350 & 1.000 & -0.545 & 0.390 & 1.000 \\
\hline & 6 & 0.113 & 0.142 & 1.000 & 0.615 & 0.311 & 1.000 & 0.545 & 0.340 & 1.000 \\
\hline & 8 & $.435^{*}$ & 0.131 & 0.043 & 0.769 & 0.343 & 1.000 & 0.818 & 0.352 & 1.000 \\
\hline \multirow[t]{7}{*}{8} & 1 & -0.516 & 0.184 & 0.186 & -1.077 & 0.348 & 0.261 & -1.273 & 0.384 & 0.218 \\
\hline & 2 & $-1.306^{*}$ & 0.137 & 0.000 & $-1.692^{*}$ & 0.286 & 0.002 & $-1.727^{*}$ & 0.333 & 0.011 \\
\hline & 3 & $-.903^{*}$ & 0.154 & 0.000 & -1.077 & 0.309 & 0.127 & -1.182 & 0.352 & 0.204 \\
\hline & 4 & $-1.468^{*}$ & 0.169 & 0.000 & $-1.846^{*}$ & 0.296 & 0.001 & $-2.000^{*}$ & 0.330 & 0.003 \\
\hline & 5 & $-1.532^{*}$ & 0.134 & 0.000 & $-1.385^{*}$ & 0.241 & 0.003 & $-1.364^{*}$ & 0.279 & 0.018 \\
\hline & 6 & -0.323 & 0.128 & 0.393 & -0.154 & 0.191 & 1.000 & -0.273 & 0.195 & 1.000 \\
\hline & 7 & $-.435^{*}$ & 0.131 & 0.043 & -0.769 & 0.343 & 1.000 & -0.818 & 0.352 & 1.000 \\
\hline
\end{tabular}

\footnotetext{
* The mean difference is significant at the 0.05 level.
} 


\subsubsection{Additional components of landscape pattern}

After scoring the eight components of landscape pattern using the Likert Scale, participants were asked to add another item to the list presented on the questionnaire, if they believed that one or more items were missing. $12.64 \%$ of the participants $(n=11)$ indicated that they would like to add another item to the list. Amongst responses, however, no new component was identified.

First, two responses were a repeat of one of the components of landscape pattern by equivalent terms or synonymous phrases available in the current literature. Using equivalent terms is commonplace in landscape ecology research (cf. Odum et al., 1971; Forman, 1995).

Second, six participants placed an emphasis on the role of matrices in affecting urban fauna. A matrix is defined as "the background cover type(s) in a landscape, characterised by extensive cover and high connectivity" (Turner et al., 2001). Turner et al. (2001) emphasise that "not all landscapes have a definable matrix” (cf. Forman and Godron, 1986; Forman, 1995). If required, however, the effects of a matrix (e.g. human disturbance) can be taken into particular consideration in a patch level under the umbrella of the two components of landscape pattern identified as connectivity and proximity, and edge density and contrast.

Third, two responses referred to the ecological history of landscape as a strong indication of the current state of biodiversity as well as the ability of land cover to be regenerated over time. Although this factor cannot be categorised under compositional or configurational dimensions of landscape pattern ${ }^{38}$, the pre-human status of landscapes should be taken into particular consideration to provide a conceptual framework for landscape restoration on an urban scale (e.g. Meurk and Swaffield, 2000; Walker et al., 2007; Chazdon and Uriarte, 2016; Schulz and Schröder, 2017).

Fourth, one participant suggested intactness as a component of landscape pattern. He/she mentioned that intactness is related to predator management. Therefore, intactness may not be directly regarded as a component of landscape pattern because it may be more related to management practices. The majority of the participants $(88.5 \%, \mathrm{n}=77)$, however, indicated that the list of the most important components of landscape pattern is acceptable.

${ }^{38}$ This topic is outside the scope of this research. 


\subsubsection{Areas of conflict or diverse functioning of urban ecosystems}

Four open-ended questions were also designed ${ }^{39}$ to address four areas of conflict when biodiversity conservation and human dimensions of climate change adaptation are concurrently addressed in terms of composition and configuration of landscape pattern (Table 2.9; cf. Section 2.2 and Table 2.3).

\section{Table 2.9}

General information on four open-ended questions addressing areas of conflict in terms of spatial patterning of landscapes in urban landscapes.

\begin{tabular}{lccc}
\hline Open-ended questions & \# of responses & \% of participation & \# of key issues frequently mentioned \\
\hline Indigenous vs. exotic & 68 & 78.16 & 57.01 \\
Spatial isolation or connection & 67 & 60.91 & 3 \\
High and low edge density and contrast & 53 & 66.66 & 3 \\
Spatially compacted or dispersed & 58 & 2 \\
\hline
\end{tabular}

\subsubsection{Question 1: Indigenous vs. exotic}

Participants were asked to address the following question and make their arguments in detail (q.v. Appendix I):

'While indigenous plant species are regarded as an essential for ecological integrity over time, some exotic plants are commonplace species for carbon sequestration and storage, and are often economically and socially acceptable. According to what you have found in the course of research and practice, is it possible to find a middle ground to alleviate the current conflict between indigenous and exotic species?’

More than $78 \%$ of the participants $(n=68)$ responded to this question seeking to ascertain whether it is possible to find a middle ground to alleviate the current conflict between indigenous and exotic flora (i.e. land cover classes) on an urban scale. This question received the highest rate of responses ( $\mathrm{n}=68$ ) amongst all of the open-ended questions. In addition, the number of key issues mentioned under this question by respondents is higher than the other three open-ended questions ( $\mathrm{n}=$ 5, cf. Table 2.9). Of the 68 participants that responded to this question, only two participants believed that finding a middle ground in this area is impossible. Importantly, six respondents mentioned that finding such a middle ground in urban landscapes is one of their research concerns and should be necessarily addressed in an era of climate change. Given that exotic species are already often available in urban landscapes, some sort of compromise between indigenous and exotic species is probably

\footnotetext{
${ }^{39}$ Detailed information has been provided in Part E of Appendix I.
} 
therefore inevitable. Amongst a range of issues mentioned in response to this question, five key issues were frequently highlighted by the participants (Table 2.10).

\subsubsection{Question 2: Spatial isolation or connection}

Participants were asked to address the following question and make their arguments in detail (q.v. Appendix I):

'While isolated patches of vegetation may be suggested to reduce the chance of pest and weed dispersal, connected patches of vegetation may facilitate the movement of species from one patch to another for foraging, breeding, roosting, etc. In an era of climate change, connected patches of vegetation can reduce the urban heat island effect through increasing the cooling effects of urban green spaces. Conversely, isolated patches of vegetation may be regarded as safe havens for wildlife species to be safeguarded against the spread of extreme weather events such as flood, heat waves, etc. According to what you have found in the course of research and practice, is it possible to find a middle ground to alleviate the current conflict between spatial isolation and connection?'

Participants were asked to express their views regarding the above-mentioned question based upon their previous experience. 67 participants (77.01\% of the sample size) answered this question. Overall, all responders except for one showed agreement on the possibility of finding a middle ground between spatial connectivity and isolation of patches of vegetation in urban areas. Three key issues were mentioned by respondents (q.v. Table 2.10).

\subsubsection{Question 3: High and low edge density and contrast}

Participants were asked to address the following question and make their arguments in detail (q.v. Appendix I):

'While low edge density can be regarded as a spatial strategy for reducing climate-related edge effects in support of wildlife species in the face of extreme weather events, high edge density can reduce the urban heat island effect through increasing the cooling effects of urban green spaces. At the same time, while high edge contrast may be a physical barrier to climate change-induced impacts such as disease spread, pest and weed dispersal, wildfire, etc., it may provide restrictions for wildlife species to move from one patch to another for foraging, breeding, roosting, etc. According to what you have found in the course of research and practice, is it possible to find a middle ground to alleviate the current conflict between high and low edge density and contrast?' 
The lowest rate of response was recorded for this question. Only 53 participants (60.91\% of the sample size) answered the question. Almost all responses focused more on the question of high or low edge contrast. Three key issues were mentioned by respondents (q.v. Table 2.10). The current literature shows that minimal research has been done on the role of edge density on human adaptation to some impacts of climate change. In addition, a long-term record of research on landscape ecology shows that there is no clear evidence or opinion on the positive and negative effects of low and high edge contrast on fauna (Botequilha Leitao et al, 2006).

\subsubsection{Question 4: Spatially compact or dispersed}

Participants were asked to address the following question and make their arguments in detail (q.v. Appendix I):

'While compact patches of vegetation may be considered as a means for reducing negative impacts on wildlife species through providing a larger core area protected from climatic edge effects, research shows that irregular extensive patches of vegetation can be beneficial for reducing the impacts of urban heat island in urban environments. According to what you have found in the course of research and practice, is it possible to find a middle ground to alleviate the current conflict between spatially compacted and dispersed configurations?’

In response to the question investigating spatially compacted or dispersed patches of vegetation in urban landscapes, $66.66 \%$ of the participants $(n=58)$, answered the question. Two key issues were mentioned by the participants (q.v. Table 2.10). 
Table 2.10

Statement excerpt depicting the most important and frequently mentioned issues by the participants.

\begin{tabular}{|c|c|}
\hline Areas of conflict & Key issues mentioned (number of participants explicitly mentioned the issue) \\
\hline $\begin{array}{l}\text { Question } 1 \\
\text { Indigenous vs. exotic }\end{array}$ & $\begin{array}{l}\text { a. The role of exotic flora in supporting indigenous wildlife species and contributing to ecosystem } \\
\text { functions cannot be set aside in urban environments ( } \mathrm{n}=21) \text {. } \\
\text { b. The question of indigenous or exotic species is species- and site-specific, and this may be } \\
\text { particularly relevant to some countries such as Australia and New Zealand ( } \mathrm{n}=12) \text {. } \\
\text { c. Exotic species, either beneficial, neutral, or detrimental, are already an integral part of urban } \\
\text { ecosystems and therefore it seems unrealistic to ignore them ( } \mathrm{n}=14) \text {. } \\
\text { d. Exotic species should be utilised only if they are non-invasive and have no negative impacts on } \\
\text { indigenous species ( } \mathrm{n}=11) \text {. } \\
\text { e. If used due to some benefits (either ecological or related to carbon sequestration), exotic species } \\
\text { should be adequately managed and carefully controlled so as not to negatively affect indigenous flora } \\
\text { and fauna. Important areas covered by indigenous flora should be protected from the invasion of exotic } \\
\text { species }(n=10) \text {. }\end{array}$ \\
\hline $\begin{array}{l}\text { Question } 2 \\
\text { Spatial isolation or connection }\end{array}$ & $\begin{array}{l}\text { a. Ecological advantages of connectivity by far outweigh its negative effects and in urban areas, as } \\
\text { elsewhere, connectivity plays a key role }(\mathrm{n}=24) \text {. } \\
\text { b. Functional connectivity is more critical than physical connection between patches of vegetation and } \\
\text { therefore, answering the question of connectivity or isolation would be extremely species-specific ( } \mathrm{n}= \\
\text { 16). } \\
\text { c. A combination of connected and isolated patches of vegetation can be a solution to meet both } \\
\text { humans and wildlife species' spatial requirements in the face of climate change impacts }(n=8) \text {. }\end{array}$ \\
\hline $\begin{array}{l}\text { Question } 3 \\
\text { High and low edge density and contrast }\end{array}$ & $\begin{array}{l}\text { a. There is a research uncertainty, lack of enough knowledge in this field of research, or the need for } \\
\text { further research in the future }(n=17) \text {. } \\
\text { b. Decisions on the importance of high or low edge contrast are species-specific }(n=12) \text {. } \\
\text { c. Benefits of low edge contrast generally outweigh advantages of high edge contrast }(n=9) \text {. }\end{array}$ \\
\hline $\begin{array}{l}\text { Question } 4 \\
\text { Spatially compact or dispersed }\end{array}$ & $\begin{array}{l}\text { a. A combination of large and compacted as well as dispersed and well-located small patches of } \\
\text { vegetation can be achievable in urban environments to meet both human needs and wildlife species } \\
\text { requirements ( } \mathrm{n}=17 \text { ). } \\
\text { b. Decision on spatially compact or dispersed patterns is species-specific }(\mathrm{n}=11) \text {. }\end{array}$ \\
\hline
\end{tabular}

\subsection{Discussion}

\subsubsection{Ranking components of landscape pattern in the context of climate change}

Climate change, rapid urbanisation, and biodiversity loss are three converging and intertwined issues of utmost importance in the twenty-first century. The ecosystem services humans need to adapt to the impacts of climate change depend on biodiversity conservation, while sometimes goals for provisioning ecosystem services through the development of green spaces in cities may contradict biodiversity conservation goals (Schwarz et al., 2017; cf. Ziter, 2016). Although spatial planning for biodiversity conservation in urban landscapes should be site- and species-specific, the overall level of biodiversity can be maintained and fostered by applying a range of basic principles, regardless of the geography, climatic zone, and the species of interest (Forman, 2014). According to the survey results, the three most important components of landscape pattern ranked by all groups are respectively patch size, connectivity and proximity, and land cover heterogeneity. This shows that there is a general 
consensus on the influence of these components on the presence, richness, and abundance of urban fauna. This finding is consistent with the results of a worldwide review conducted by Nielsen et al. (2014) that demonstrated the importance of habitat diversity (equal to land cover heterogeneity), connectivity (equal to connectivity and proximity), and patch size in urban parks as crucial factors affecting a wide range of vertebrates, with particular emphasis on avifauna, small mammals, and reptiles that typically coexist with humans in cities. In addition, a meta-analysis of 75 cities worldwide suggests that patch area (i.e. patch size) and corridors (i.e. connectivity and proximity) are the most important factors to support urban biodiversity (Beninde et al., 2015). The present research, however, quantitatively measures and weights the importance of each component of landscape pattern and provides a comparable ranking based upon what researchers have experienced and observed through their studies as well as their knowledge of the literature in the context of climate change. This research also reveals how and to what degree the opinions of researchers with experience in the Southern Hemisphere and Oceania may influence a global ranking of the most important components of landscape pattern. Furthermore, the research is not confined to the patch level (e.g. urban parks), per se, but also addresses biodiversity at the urban landscape level.

In the five other components of landscape pattern, there is a difference between the weights of importance assigned to each component by each group of participants. The most obvious difference is the dedication of a higher weight to indigenousness by Groups B and C. This can be best explained by acknowledging the regional status of biodiversity loss and ecosystem degradation in the Southern Hemisphere and Oceania (Craig et al., 2000; Villavicencio et al., 2016; van Wilgen et al., 2016). Given that many Southern Hemisphere countries are currently experiencing severe indigenous biodiversity loss as a result of human colonisation and its ongoing side effects (Elton, 1958; Morton, 1990; Cowling et al., 1996; Craig et al., 2000; Pauchard et al., 2006; Villavicencio et al., 2016; van Wilgen et al., 2016), the involvement of participants who have had rich empirical experience on urban biodiversity issues in Southern Hemisphere countries was extremely important in this research to show how their viewpoints and opinions may influence the current global perspective. For example, research shows that the loss of indigenous biodiversity as a result of climate change in the Southern Hemisphere in general, and in Oceania in particular, is acute (Kingsford et al., 2009; Jupiter et al., 2014; Urban, 2015; Taylor and Kumar, 2016). Another challenge, according to Chambers et al. (2017), is the lack of, or insufficient, long-term ecological data in Southern Hemisphere countries compared to North America, Europe, and Asia. Research shows that Southern Hemisphere nations are amongst those countries that host the world's most abundant, rich, and endemic flora and fauna (Myers et al., 2000; Olson et al., 2001). 
In the case of other components of landscape pattern (i.e. land surface perviousness, edge density and contrast, landform diversity, and shape complexity), there is a slight difference in ranking by each group. In this case, all groups have ranked shape complexity as the least important component of landscape pattern in urban landscapes.

In terms of differences in assigned weights between groups, the highest difference can be observed between Groups A and C, whereas the lowest difference is between Groups B and C (q.v. Table 2.5). This may be explained in relation to similar challenges and issues that biodiversity faces in the Southern Hemisphere compared to the world as a whole. According to Myers et al. (2000), of the world's 25 biodiversity hotspots, four (16\%) and ten (40\%) of the hotspots are in Oceania and the rest of the Southern Hemisphere, respectively. Olson et al. (2001) classify Oceania as one of the world's eight most important ecoregions. More specifically in the case of New Zealand, Meurk et al. (2016) argue that the country developed a unique ecology as a result of long-term geological isolation. Craig et al. (2000) provide ample evidence that indigenous biodiversity in New Zealand has widely suffered as a result of anthropogenic development. Almost all countries in this region, including Australia and New Zealand are experiencing rapid urban population growth, as well (Dyball et al., 2013; United Nations, 2014). This, accordingly, accelerates the rate of biodiversity loss in urban landscapes.

With reference to Tables 2.5 and 2.6, there is a worldwide consensus among researchers on the integrated role of patch size, land cover heterogeneity, and connectivity and proximity in affecting the overall level of biodiversity in urban landscapes. Therefore, these components are critically important to biodiversity conservation, irrespective of the geographical location or species of interest. Interestingly, the importance of indigenousness is significantly lower than land cover heterogeneity, patch size, and connectivity and proximity for Group A. Although the importance of indigenousness is similarly lower than the aforementioned components of landscape pattern for Groups B and C, mean differences are not statistically significant $(p>0.05)$. This means that in line with the first three components of landscape pattern, indigenousness should also be concurrently taken into account to conserve biodiversity in urban landscapes of the Southern Hemisphere and particularly in Oceania. For example, the importance of areas covered by indigenous land cover classes (i.e. indigenousness) has been taken into particular attention as an important factor in the process of site selection of wildlife sanctuaries in urban New Zealand (q.v. Chapter 8). Shape complexity was found to be the least important component of landscape pattern in all groups. Rankings provided by Groups B and C do not affect the position of this component in the global ranking provided by Group A. Although the importance of this component is thought to be lower than other components of landscape pattern in all 
groups, not all differences are statistically significant. In Group A, mean differences between shape complexity and indigenousness and edge density and contrast do not significantly differ. In the case of Group B, the importance of shape complexity is significantly lower than land cover heterogeneity, patch size, and connectivity and proximity. In Group C, it is only patch size that is significantly different from shape complexity. From this, it can be deduced that the spatial configuration of patches of vegetation is particularly important in Australia, New Caledonia, and New Zealand.

\subsubsection{Areas of conflict between humans and fauna}

Based upon Table 2.3, there are four areas of conflict between humans and fauna in urban landscapes when the spatial patterning of patches of vegetation is the core topic of interest (cf. Table 2.10). The most discussed topic in the four open-ended questions belongs to the question of indigenous vs. exotic. This is a topic that is extremely significant in the urban landscapes of the Southern Hemisphere and Oceania (Meurk and Swaffield, 2000). Specifically in urban New Zealand, recent research shows that some exotic flora does support indigenous fauna (Gray and van Heezik, 2016). The general belief, however, is that while exotic species may be beneficial, they should be utilised only if they are non-invasive and have no negative impacts on indigenous species.

Although respondents have provided a range of valuable recommendations in response to the four open-ended questions (q.v. Table 2.10), they frequently argued that addressing such areas of conflict are species- and site-specific and that to attain the most desirable outcomes, general recommendations should be considered in regard to the ecological status of sites and the nature of the local impacts of climate change on species of interest to decrease the odds of further indigenous biodiversity loss. Collectively, respondents to the four open-ended questions mentioned 48 times that answering such questions depends largely on the site and species of interest. This relatively high rate of frequency shows that despite general recommendations provided by participants based upon their empirical research experience, there is always a need for a local oriented view on biodiversity issues (Turner, 1989; Ahern, 1999; Botequilha Leitao and Ahern, 2002; Botequilha Leitao et al, 2006). Overall, according to the responses collected by the four open-ended questions, it seems that having a combination of strategies in different places (i.e. a number of networks of patches of vegetation for various purposes to support both humans and fauna) can possibly help to achieve a middle ground across the whole landscape. Anthropogenic factors such as economic and social drivers cannot be neglected in the process of landscape planning and management in areas suffering from urbanisation. Socio-economic factors play a major role in the spatial patterning of landscapes in human-occupied landscapes worldwide (Meyer and Turner, 1992; Lambin et al., 2001; Xiao et al., 2006; Groffman et 
al., 2014). One the other hand, intact ecosystems are becoming increasingly rare in cities (q.v. Chapter 7). Therefore, it is essential to begin to address biodiversity requirements in the face of climate change alongside land-based policies that are being applied to adapt settlements and humans to climate change. Although research has begun to use urban green spaces as multi-purpose vehicles to concurrently safeguard both biodiversity and humans against some of the impacts attributed to climate change (Wilby and Perry, 2006), there still seems to be research uncertainties and lack of enough knowledge to meet both human needs and wildlife requirements through spatial patterning of patches of vegetation (q.v. Table 2.10).

In light of this research, five recommendations can be offered as general guidelines for landscape planning and management in support of urban biodiversity in the face of climate change impacts, while simultaneously considering areas of conflict (q.v. Tables 2.3 and 2.10).

First, patch size, connectivity and proximity, and land cover heterogeneity are the three most important components of landscape pattern affecting fauna in an era of climate change. In the Southern Hemisphere and Oceania, indigenousness is the next most important factor that should be concurrently taken into consideration.

Second, while the overall level of biodiversity can be maintained and/or improved by applying a range of strategies discussed in this research, urban biodiversity research remains largely site- and species-specific. Thus, information about the local impacts of climate change and species of interest is required to make informed decisions on how to intervene in the spatial composition and configuration of patches of vegetation.

Third, while larger patches of vegetation are likely to play a key role in supporting urban fauna in the face of climate change, the supplementary role of small patches should not be set aside because they may be considered as stepping stones by some mobile species (i.e. avifauna) and therefore can provide functional connectivity between isolated larger patches of vegetation. For this reason, a spectrum of patches of various sizes is needed to meet the ecological requirements of urban fauna.

Fourth, although functional connectivity is critically important to facilitate the movement of species across landscapes, a small number of isolated patches of vegetation combined with suitable landforms consisting of a range of aspects of slopes may also be considered as important climatic refugia in the event of unforeseen extreme climatic conditions. 
Fifth, to decrease areas of conflict between humans and fauna, site- and species-specific informed interpretation of information provided in Study I can be utilised depending on the issue of concern in different parts of the world (q.v. Table 2.10).

As depicted in Table 2.10, this may appear spatially as a multi-layer network of patches of vegetation in different shapes and sizes, with different levels of connectivity including a number of isolated patches integrated with both compact and elongated patches of vegetation that can concurrently meet fauna and human needs in a more holistic manner. This provides spatially sustainable habitats and food sources for fauna and concurrently helps humans to adapt to the impacts of climate change through a wide range of ecosystem services increased by informed interventions in the spatial composition and configuration of patches of vegetation. For example, as shape complexity is considered the least important component of landscape pattern while patch size is believed to be the most important factor when planning for biodiversity conservation in urban landscapes, a large patch of vegetation with a number of dispersed corridors connected to the main body of the patch could be regarded as an optimised pattern to serve as a suitable interior habitat protected from edge effects for fauna. Concurrently this provides a higher level of evapotranspiration and cooling effects across the landscape through connected vegetation corridors to support people who face urban heat island effects triggered by rising temperatures. This is because such spatial patterns are associated with higher edge density. Further research is required to test issues discussed in specific urban landscapes and in regard to specific species under the local impacts of climate change.

\subsection{Conclusion}

Study I provides an expert-driven ranking of the most important components of landscape pattern affecting fauna in urban landscapes and reflects expert knowledge gained in the course of at least 325 research projects in 39 countries between 2000 and 2017. Results provide original data for global biodiversity research including habitat suitability analysis and site-selection for identifying the most spatially suitable patches of vegetation to serve as habitats for fauna. The research also reveals that the opinions of researchers with experience in the Southern Hemisphere influence the global ranking of the most important components of landscape pattern. Furthermore, a small difference between the Southern Hemisphere and Oceania was observed.

This model of ranking was provided in relation to climate change impact awareness because participants were asked to assign appropriate scores to each component of landscape pattern with particular attention to the effects of climate change on urban fauna. Data provided by this survey can 
potentially be a viable compromise for the lack of enough empirical data because results derived from the survey generate useful information for safeguarding fauna against some of the ill-effects of climate change in urban landscapes. The scale of the study, the rate of participation in the survey, and more importantly the knowledge and experience quality of participants, may ensure that the results and recommendations extrapolated from them are a reliable source of information pending the availability of relevant empirical data. The next chapter will discuss how this information can be applied to a New Zealand context (q.v. Chapter 3). 


\section{CHAPTER 3}

Study II: Semi-structured interviews with New Zealand subject-matter experts

\subsection{RESEARCH AIMS}

Chapter 3 reports on Study II. This study was conducted to fill the current information gap in the literature (q.v. Section 1.2.4; Figure 1.10), to contribute to a more robust and reliable dataset to be utilised in Study III (q.v. Chapters 4-8). In particular, this study was conducted to:

(1) Confirm and adjust the results derived from Study I to be applied to a New Zealand context (q.v. Chapter 2);

(2) Weight and rank the local impacts of climate change on the Wellington urban landscape based upon their potential effects on urban wildlife habitats as well as fauna with particular emphasis on the spatial ecology of the keystone species (q.v. Table 1.4);

(3) Consider the spatial thresholds previously defined by New Zealand researchers as a basis for judgment about the capability of the current landscape patterns in safeguarding indigenous fauna against the ill-effects of climate change;

(4) Obtain a range of biodiversity data that is unlikely to be found in the current literature including information about the possibility of increasing land cover heterogeneity through natural regeneration mechanisms.

\subsection{MATERIALS AND METHODS}

To accomplish the research aims of Study II, six questions were designed based upon the available data published in the current literature as well as the results derived from Study I (q.v. Chapter 2; Appendix III). Standards of qualitative research were carefully observed with particular 
emphasis on interview techniques (Briggs, 1986; Gubrium and Holstein, 2002; Silverman, 2004; DiCicco-Bloom and Crabtree, 2006; Potter and Hepburn, 2012; Robinson, 2014; Castillo-Montoya, 2016). More specifically, a number of interview-centred urban biodiversity studies were scrutinised to design a robust framework for this study (Yli-Pelkonen and Niemelä, 2006; Hagerman et al. 2010; Saarela and Rinne, 2016). Study II's materials and methods were approved by the Victoria University of Wellington Human Ethics Committee on 6 March 2017 (Appendix IV).

Question 1 was set up to discuss and finalise the weighted factors (i.e. the eight components of landscape pattern) to be used in the process of urban wildlife sanctuary site selection in Study III (q.v. Chapter 8). Question 2 was designed to check the accuracy of information extracted from the literature, summarised in Table 1.4. The aim of Question 3 was to address the potential for increasing land cover heterogeneity in the study area based upon the standard land cover classification system for New Zealand provided by Landcare Research (2015). Questions 4 and 6 were designed to provide input data for landscape computation using FRAGSTATS (q.v. Chapter 7). Question 5 was set up to rank the local impacts of climate change to provide a context for landscape pattern analysis and interpretation in Study III with particular attention to information provided in Table 1.5 and Sections 1.4.2 to 1.4.4.

Online databases of international repute were searched in order to find the relevant subjectmatter experts based upon their publications published since 2000. Researchers with a track record of publications in peer-reviewed journals were targeted. Keywords were used to find relevant publications and thereby researchers. A systematic search was conducted in internationally recognised databases (e.g. Scopus, ISI Web of Knowledge, etc.). The websites of universities and research centres were also explored to ensure that no one was omitted from the search. Eleven potential participants were retrieved using these methods. Ultimately, six subject-matter experts from Wellington ( $\mathrm{n}=3)$, Christchurch $(\mathrm{n}=2)$, and Dunedin $(\mathrm{n}=1)$, with long-term track records of research on spatial planning for biodiversity conservation in urban New Zealand, accepted invitations to contribute to the research. Interview materials were separately sent to the six participants at least one week in advance of the interview session. Interview materials consisted of (1) interview questions, (2) interview information sheet, and (3) interview consent form. 
Interviews were conducted from 21 March to 24 May $2017^{40}$. Interviews took between 35 and 105 minutes depending on the nature of discussions and the number of additional questions raised based upon the interviewee's responses to the six main questions. Although answering all questions was optional, all participants answered all questions. Responses and discussions were recorded using a voice recorder after permission from the interviewees was given. They were free to stop the interview at any time, without giving a reason. In addition, they could withdraw from the study by contacting the researcher at any point before 30 September 2017. Participants were invited to answer two out of six questions in writing in advance of conducting the interview (viz. Questions 4 and 5). They were asked to return their written responses before or after the interview session in person or via email.

After converting each interview into text, a summarised written version of it was returned to each participant on 18 June 2017 for final revision. Each participant had the opportunity to revise his/her statements no later than 25 June 2017.

Finally, the aggregated results were sent to all interviewees for final revision. Each participant had an opportunity to compare his/her responses to the report, anonymously. Given that Study II was confidential, the collected data was aggregated, and participants' identities were not disclosed in any reports, presentations, or public documentation.

\subsection{RESULTS AND DISCUSSION}

\subsubsection{Interview questions and responses}

\subsubsection{Question 1: Components of landscape pattern in a New Zealand context}

In Study I, the most important components of landscape pattern affecting the presence, richness, and abundance of fauna in urban landscapes were ranked by international scholars (q.v. Chapter 2). In Study II, the New Zealand interviewees were asked to consider if the rankings derived from Study I are relevant to a New Zealand context and could be applied to landscape architecture practices in urban New Zealand. They were asked to consider two factors in advance of answering the question:

(1) The spatial ecology and requirements of the selected keystone species currently present, or likely to be present, in the study area (q.v. Table 1.4);

\footnotetext{
${ }^{40}$ Four participants agreed to take part in a face-to-face interview (two in their offices and two at the Victoria University of Wellington's Faculty of Architecture and Design), while two participants agreed to be interviewed remotely using the Skype programme.
} 
(2) The estimated impacts on the selected keystone species attributed to climate change in the current literature (Table 3.1; cf. Table 1.5).

All six interviewees argued that the rankings provided by international researchers can be applied to a New Zealand urban context because biodiversity issues are, to a large extent, globally widespread and the current problems are similar in many parts of the world (cf. Pereira et al., 2010; Mora and Sale, 2011; Hooper et al., 2012; Joppa, 2016). More specifically, they argued that patch size, connectivity and proximity, and land cover heterogeneity could be considered the most important components of landscape patterns affecting indigenous fauna in urban New Zealand (cf. Section 2.4.2). The interviewees were also asked to explain why a higher weight has been dedicated to indigenousness by researchers with experience in research on biodiversity in the Southern Hemisphere and Oceania (q.v. Section 2.4.2.2). From the international literature, for example, Forman (1995) suggests maintaining patches of indigenous vegetation, as small bits of nature, in humanoccupied landscapes because the services they provide as primary habitats and/or stepping stones for a wide range of fauna are important. Indigenous flora have an irreplaceable role in biodiversity - and thereby long-term ecosystem integrity in cities (Aronson et al., 2014). Fischer et al. (2009) stated that the overall level of biodiversity depends on the total number of indigenous flora present in a landscape.

Table 3.1

Examples of probable consequences of local impacts of climate change on wildlife habitats in the Wellington urban landscape (cf. Table 1.5).

Climate change impacts

Direct impacts

- Wildlife habitats specifically along edges are subject to extreme weather events such as intensified windstorms;

- Some urban wildlife habitats are susceptible to extreme weather events triggered by rising temperatures and intensified windstorms, especially along edges;

- Low-land areas are susceptible to flooding and inundation affecting remaining habitats within the city;

- Wildlife habitats may get warmer, specifically in north-facing slopes, providing unsuitable conditions for indigenous fauna historically adapted to normal temperatures;

- $\quad$ Large areas of urban costal habitats are subject to sea level rise affecting seabirds.

\section{Indirect impacts}

- Extreme weather events such as intensified windstorms and flooding events may facilitate the increased dispersal of weeds and pests affecting indigenous flora and consequently fauna, specifically along edges;

- $\quad$ Rising temperatures may benefit exotic fauna, particularly predators of indigenous fauna. This may increase the rate of predation upon New Zealand avifauna and reptiles;

- More frequent heavy rainfalls can give rise to more landslides that will destroy or damage wildlife habitats specifically in areas where landform diversity can harbour indigenous fauna;

- $\quad$ The outbreak and spread of diseases in avifauna is likely to be higher in warmer than average temperatures;

- Sea level rise may reinforce the need to relocate different types of urban infrastructure (e.g. industrial, commercial, recreational, etc.) to new open spaces along the water bodies and this could negatively affect indigenous species that depend on coastal habitats. 
The direct relationship between the percentages of indigenous vegetation and the extinction rates of indigenous plant species has been confirmed in urban landscapes (Hahs et al., 2009). The interviewees' arguments infer that indigenousness must concurrently be regarded as an important component of landscape pattern, not necessarily after the three aforementioned components (i.e. patch size, connectivity and proximity, and land cover heterogeneity), but in tandem with them. This attitude can be strongly linked to the scarcity of patches of indigenous vegetation and accordingly loss of indigenous flora in urban New Zealand in general (Given and Meurk, 2000; Meurk and Swaffield, 2000; Freeman and Buck, 2003) and in the Wellington urban landscape in particular (Bagnall, 1979; Clarkson et al., 2007; Pedersen Zari, 2012).

The interviewees believed that indigenous land cover is important for the survival of fauna in the Southern Hemisphere in general and New Zealand in particular because the landmass of New Zealand was isolated for more than eighty-five million years (cf. Section 1.1.5). This is because of the importance of vital ecological interactions between indigenous ecosystems and indigenous fauna (q.v. Chapter 1). One of them, for example, argued that it is idiosyncratic with regards to a number of important factors including long isolation from the rest of mainstream revolutions, unique biogeography, and long history without the presence of mammals. New Zealand indigenous fauna include flightless birds, reptiles, and large insects. They have minimal, if any natural defence against introduced predators. New Zealand is the last continent / island group to be occupied by humans. In light of this, and the unique characteristics of indigenous biodiversity, hunting, habitat modification, and introducing predators have widely affected New Zealand indigenous biodiversity (cf. Allen and Lee, 2006). The most important issues discussed in addressing Question 1 have been summarised in Table 3.2. 
Table 3.2

Interview excerpts depicting the significance of indigenous biodiversity in urban New Zealand.

\begin{tabular}{ll}
\hline Item & The most important issues mentioned by the interviewees \\
\hline A & $\begin{array}{l}\text { In general, the ranking provided by international researchers can also be applied to a New Zealand context because } \\
\text { biodiversity loss is a global issue and principles of landscape ecology science are, to a large extent, similar in different } \\
\text { environments. }\end{array}$ \\
B & $\begin{array}{l}\text { New Zealand's long-term geographical isolation from other continents has made the country not only unique, but } \\
\text { ecologically fragile. }\end{array}$ \\
C & $\begin{array}{l}\text { Continued human-caused ecosystem degradation and habitat loss have had comprehensive impacts on indigenous } \\
\text { biodiversity in urban New Zealand, perhaps more than other parts of the world. }\end{array}$ \\
D & $\begin{array}{l}\text { The presence of introduced fauna and invasive flora is a major problem in the Southern Hemisphere while many } \\
\text { countries is threatened more severely. In New Zealand in particular, many urban landscapes have been dominated by } \\
\text { exotic and invasive flora, which may be due to important factors including cultural backgrounds and colonisation } \\
\text { trends over history. } \\
\text { E }\end{array} \quad \begin{array}{l}\text { In line with the significance of composition and configuration of patches of vegetation in urban landscapes, weed and } \\
\text { pest control are also necessary to protect indigenous fauna from increased predator pressures. }\end{array}$ \\
F & $\begin{array}{l}\text { Given that New Zealand cities are home to a high percentage of exotic plant species, already present non-native species } \\
\text { could be managed to provide supplementary food sources for indigenous fauna, if they are not invasive. }\end{array}$ \\
\hline
\end{tabular}

\subsubsection{Question 2: Keystone species under study}

Reviewing the literature, an initial table of keystone species was presented to the interviewees (q.v. Table 1.4). They were asked to consider if any other keystone species not listed in the table should have been included. The concept of keystone species was applied to be more site- and speciesspecific in the course of Study III (q.v. Chapters 4-8).

Excluding the little penguin that is widely dependant on coastal areas in Australia and New Zealand for nesting, moulting, and breeding (Boersma, 2008), the other species are endemic to New Zealand $^{41}$. All interviewees mentioned that from both cultural and ecological perspectives, the list of the selected keystone species is generally acceptable. Beyond this, one of them believed that if suitable conditions are provided for them, the study area would have flourishing ecosystems.

Four interviewees argued that some important invertebrates such as the Wellington tree weta (Hemideina crassidens) could also be included on the list. In addition, two interviewees underscored the role of kākā (Nestor meridionalis) in the composition and ecology of forests. They stated that kākā are very important as a keystone species due to their role in pollination and seed dispersal. Despite

\footnotetext{
${ }^{41}$ Detailed information about habitat requirements of the little penguin and the relationship between its behaviour and spatial composition and configuration of its habitats can be found in Paper III (q.v. Publications Information).
} 
this, they highlighted the fact that while kākā do not need very large habitats, predator free patches of indigenous vegetation are required to support this species (cf. Wilson et al., 1998). Furthermore, recent research shows that the reintroduction of kākā to the Wellington urban landscape has been associated with an increased human-wildlife conflict (Charles and Linklater, 2015). One of the interviewees argued that takahē (Porphyrio hochstetteri) and rifleman (Acanthisitta chloris) may be also added to the list of selected keystone species. Takahē and rifleman are extremely vulnerable to exotic predators (Bunin and Jamieson, 1996; Starling-Windhof et al., 2011). In addition, one of the interviewees suggested the fantail (Rhipidura) as another keystone species in urban New Zealand.

Four out of six interviewees argued that tuatara and long-tailed bats are indicators of ecosystem health, but are not necessarily keystone species, at least from an ecological point of view. In addition, one of the interviewees believed that the short-tailed bat (Mystacina tuberculata), as an indigenous pollinator, is more likely to be reintroduced to the Wellington urban landscape. This argument was supported by another interviewee who suggested that the presence of long-tailed bats in the Wellington urban landscape seems unlikely because the plants they need for habitat selection and foraging are rare in this region. Despite this, they confirmed that the aforementioned species are culturally important because they are genetically unique and endemic to New Zealand (cf. Garibaldi and Turner, 2004). The same argument was made for little-spotted kiwi by one of the interviewees. One of the interviewees also suggested that the little penguin is not an ecological keystone species, but its presence is critically important in coastal areas because little penguins are indicative of the health of the other marine species (Moller et al., 2000; Flemming, 2013). Finally, it was suggested that the New Zealand falcon (Falco novaeseelandiae) be added to the list of keystone species by one of the interviewees for their key role in food chains.

Overall, there seems to be a consensus on the ecological importance of four species of avifauna (viz. bellbird, hihi, kererū, tūî) and two species of reptiles (viz. forest gecko and Wellington green gecko), as the most important keystone species in the Wellington urban landscape, whereas there is a divergence of opinions on the inclusion of long-tailed bat, tuatara, little-spotted kiwi, and little penguin in the list of indigenous keystone species. Based upon the literature (q.v. Chapter 1), and as confirmed by results stemming from the interviews, the final list of the selected keystone species in the study area is provided in Table 3.3.

There is an integrated mutual relationship between indigenous ecosystems and these keystone species in New Zealand. Ecosystems provide primary habitats and food sources for keystone species and they help ecosystems to regenerate over time through seed dispersal and pollination processes 
(q.v. Section 1.4.3). Keystone species listed in Table 3.3 effectively function as pollinators and seed dispersers of at least 70\% of forest species of woody plants in urban and non-urban New Zealand (q.v. Chapter 6). They play a vital and irreplaceable role in supporting the healthy functioning of ecosystems in New Zealand. Some of these keystone species, including kererū and tūî in particular, can concurrently serve as indictors of successful mammalian pest control (Baber et al., 2009; MacLeod et al., 2015). There is a consensus on this list amongst the interviewees, and therefore, the list was regarded as a basis for the research in Study III (q.v. Chapters 4-8).

Table 3.3

Final list of the selected keystone species in urban New Zealand confirmed in Study II (cf. Table 1.4).

\begin{tabular}{ll}
\hline Keystone species present in the study area & Scientific name \\
\hline Indigenous avifauna & \\
Bellbird & Anthornis melanura \\
Hihi & Notiomystis cincta \\
Kererū & Hemiphaga novaeseelandiae \\
Tūī & Prosthemadera novaeseelandiae \\
& \\
Indigenous reptiles & \\
Forest gecko & Hoplodactylus granulatus \\
Wellington green gecko & Naultinus elegans punctatus \\
\hline
\end{tabular}

\subsubsection{Question 3: Potentials for increasing land cover heterogeneity}

According to Study I (q.v. Chapter 2), land cover heterogeneity is believed to be the second most important component of landscape pattern affecting urban fauna in an era of climate change (q.v. Section 2.4.2). Of thirty-three land cover classes in the New Zealand environment (Landcare research, 2015; q.v. Appendix V), sixteen are currently present in the study area (q.v. Chapter 4). The interviewees were asked if the study area is capable of greater land cover heterogeneity (q.v. Table 2.1). They were also encouraged to expand their responses to explain how this can be attainable in an urban New Zealand context. A robust consensus was observed affirming the possibility of increasing the number of land cover classes in the Wellington urban landscape by applying landscape restoration practices, including both natural regeneration mechanisms and human interventions in current landscape patterns. No contradictions were observed in the interviewees' viewpoints. More specifically, the key role of the exotic class Gorse and/or Broom was highlighted by five out of six interviewees as important nursery plants for the beginning of ecological succession towards the class Indigenous Forest (Davis and Meurk, 2001; Williams, 2011; Wotton and McAlpine, 2013; Landcare Research, 2015; q.v. Chapter 6; Appendix V). This, accordingly, underscores the importance of developing knowledge of landscape restoration in urban New Zealand through natural regeneration processes (cf. Clarkson and Kirby, 2016). In particular, two indigenous classes, namely (1) Fernland and (2) Manuka and Kanuka that are currently absent in the study area, were suggested by four 
interviewees as potential land cover classes that could be regenerated at a large scale through ecological succession processes in the Wellington urban landscape (q.v. Appendix V).

\subsubsection{Question 4: Edge contrast between land cover classes}

Each interviewee was asked to complete the dissimilarity matrix (Table 3.5) based upon his/her New Zealand-based empirical experience of different groups of land cover classes (Table 3.4). The available literature shows that high edge contrast may have either positive or negative impacts on urban fauna depending on the site and species under investigation (Sattler et al., 2010; Noreika and Kotze, 2012; Ikin et al., 2013). The interviewees were invited to indicate a dissimilarity (edge contrast) weight, using a score from 1.0, the highest edge contrast, to 0.0 , the lowest edge contrast, in terms of its unique influence on the selected keystone species (q.v. Table 3.1) with a particular attention to the local impacts of climate change (q.v. Section 1.4.2). For example, if an interviewee believed that the dissimilarity between Type A and Type C is high, he/she could give a score of 1.0 and conversely, if he/she believed that the dissimilarity has minimal or no negative effect on the relevant keystone species, he/she could give a score of 0.0 .

The interviewees were provided with a spectrum to give edge contrast weights (Figure 3.1). They were also asked to answer this question in writing and return their responses via email within two weeks from the date of the interview session. According to the aggregated responses, the highest and lowest edge contrast weights were between Types A and C (0.9), and Types A and B (0.425), respectively (q.v. Table 3.5). Despite this, all interviewees stated that the dedication of edge contrast is highly species-specific. In addition, three out of six interviewees argued that edge contrast has the least impact on the selected keystone species. This can be explained through two lines of argument. First, bellbird, hihi, kererū, and tūī are avifauna and therefore, urban streets and buildings are not serious physical barriers to restrict the movement of these species. Second, the home range size of the selected reptiles (viz. forest gecko and Wellington green gecko) is very small and for this reason, even very small patches of vegetation can provide the species with enough primary habitat and food sources without there being a need for movement from one patch to another (cf. Whitaker, 1987; Wotton, 2002) (Figure 3.2).

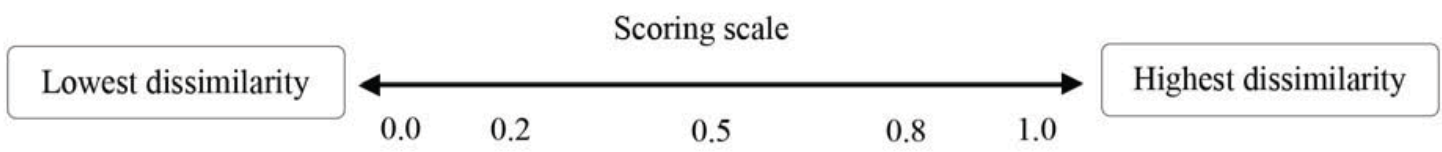

Figure 3.1. Scoring scale used to dedicate dissimilarity weights to each pair group of land cover classes (cf. Table 3.5). 


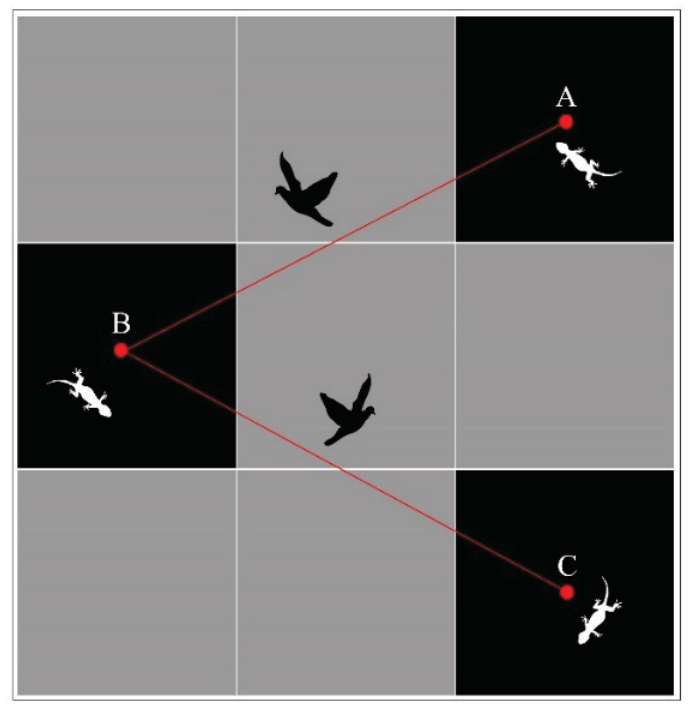

Figure 3.2. A conceptual illustration of home range size and the movement of avifauna in and between habitat patches (black) in an urban matrix (grey), and reptiles within habitat patches (not to scale).

Some species were removed from the initial list of the selected keystone species (viz. longtailed bat, little-spotted kiwi, tuatara, little penguin), due to either their absence in the study area (viz. long-tailed bat), the lack of widespread presence (viz. little penguin), or the inevitable confinement in pest-free urban wildlife sanctuaries (viz. little-spotted kiwi and tuatara). Thus, this study suggests that quantifying and measuring edge contrast in relation to the spatial requirements of the selected keystone species would be unnecessary in the study area $^{42}$. Despite this, information on edge contrast weights provided in the course of interviews has been presented (q.v. Table 3.5). Although subjective, weights provided by the interviewees can be considered as a starting point for detailed empirical research on the role of edge contrast on urban fauna in New Zealand in the future. ${ }^{42}$ For this reason, hereafter, the term edge density and contrast is revised and instead termed edge density (q.v. Tables 1.1
and 2.1). 
Table 3.4

Sixteen land cover classes in the study area classified in terms of general ecological characteristics

\begin{tabular}{ll}
\hline Groups & Land Cover Classes \\
\hline Type A: Indigenous & Broadleaved Indigenous Hardwoods \\
& Indigenous Forest \\
& Matagouri or Grey Scrub \\
& Herbaceous Freshwater Vegetation \\
Type B: Non-indigenous & \\
& Gorse and/or Broom \\
& High Producing Exotic Grassland \\
& Exotic Forest \\
Type C: Extremely human-modified & Urban Parkland/Open Space \\
& Low Producing Grassland \\
& Forest Harvested \\
Type D: Other & Built-Up Area \\
& Transport Infrastructure \\
& Surface Mine or Dump \\
\hline
\end{tabular}

Table 3.5

The dissimilarity matrix constructed based upon the interviewees' long-term experience on biodiversity issues in urban New Zealand (cf. Table 3.4).

\begin{tabular}{lcccc}
\hline Edge contrast (0-1) & Type A & Type B & Type C & Type D \\
\hline Type A & 0.000 & 0.425 & 0.900 & 0.633 \\
Type B & 0.425 & 0.000 & 0.704 & 0.658 \\
Type C & 0.900 & 0.704 & 0.000 & 0.500 \\
Type D & 0.633 & 0.658 & 0.500 & 0.000 \\
\hline
\end{tabular}

\subsubsection{Question 5: Ranking the local impacts of climate change}

According to middle of the range estimations provided by the New Zealand Ministry for the Environment (2014), at least five impacts can be attributed to climate change in the Wellington Region, and accordingly the Wellington urban landscape (q.v. Section 1.4.2). According to the abridged information about the relationship between climate change impacts and the selected keystone species (q.v. Tables 1.5 and 3.1), the interviewees were asked to rank the estimated impacts of climate change based upon the depth and extensiveness of the impacts on the keystone species and their habitats from the most detrimental to the least detrimental using the Likert Scale (Deming and Swaffield, 2011). Similar to Question 4, the interviewees could answer this question in writing and return their responses via email within two weeks from the date of interview session. The overall ranking of the local impacts of climate change provided by the interviewees shows that storms (score $=18$ ), rising temperatures (score $=17$ ), and change in rainfall patterns (score $=16$ ) are estimated to have the most detrimental impacts on the selected keystone species. Changes in wind patterns (score $=13$ ) and sea level rise (score $=12$ ) are ranked next. Given that storm events are associated with 
severe winds, a coupled impact imposed by wind and storms as well as rising temperatures seems likely to have the most detrimental impacts on the selected keystone species according to the six subject-matter experts. Although empirical data about the relationship between the local impacts of climate change and the selected keystone species is still insufficient (q.v. Chapter 1), there is evidence in New Zealand to confirm that the assertions made by the interviewees are, to a large extent, reliable (cf. McGlone and Walker, 2011; Christie, 2014).

\subsubsection{Question 6: Spatial thresholds}

With particular attention to the discussed keystone species' ecological requirements as well as the local impacts of climate change on them, the interviewees were invited to express their opinions about the proposed spatial thresholds suggested by New Zealand researchers (Table 3.6). The spatial thresholds are indicative of the minimum spatially acceptable level of landscape pattern safeguarding, or at least supporting urban fauna against the ill-effects of climate change in urban New Zealand. In this research, the spatial thresholds have been linked to the eight main components of landscape pattern. Meurk and Hall (2006) argue that this set of spatial thresholds can support a wide range of urban fauna in New Zealand urban landscapes and at the same time, facilitate key mechanisms such as pollination and seed dispersal (cf. Meurk and Swaffield, 2007; Ignatieva et al., 2011).

Table 3.6

Spatial thresholds proposed for supporting indigenous biodiversity in urban New Zealand (Meurk and Hall, 2006) linked to components of landscape pattern defined in the current research.

\begin{tabular}{|c|c|}
\hline Components of landscape pattern & Spatial thresholds \\
\hline Indigenousness & At least $20 \%$ of the total landscape \\
\hline Land cover heterogeneity & Not defined \\
\hline Land surface perviousness & Not defined \\
\hline Patch size & A network of patches $\geq 6.25$ ha, $\geq 1.56$ ha, and $\geq 0.01$ ha \\
\hline Connectivity and proximity & Between $<200 \mathrm{~m}$ and $<5000 \mathrm{~m}$ depending on the size of patches of vegetation \\
\hline Edge density and contrast & At least a $50 \mathrm{~m}$ buffer zone around the patch of indigenous vegetation \\
\hline Landform diversity & Not defined \\
\hline Shape complexity & As compact as possible, no quantitative data \\
\hline
\end{tabular}


The spatial thresholds have the potential to provide a platform for addressing two important questions:

(1) Do the proposed spatial limits or size thresholds stand the test of time in an era of climate change regarding the habitat requirements of keystone species?

(2) Can new size thresholds be proposed for those components of landscape pattern that have received no attention? ${ }^{43}$

While the first question addresses the validity of the thresholds in an era of climate change, the second question seeks to propose thresholds for three components of landscape pattern, namely land cover heterogeneity, land surface perviousness, and landform diversity.

The interviewees were informed that the responses will be normalised in order to construct a basis for examining the current landscape pattern in the study area in order to make informed judgements about the capability of landscape pattern composition and configuration in safeguarding indigenous biodiversity in the face of climate change impacts. Additional information regarding the spatial thresholds was provided for the interviewees in the interview session to provide a basis for more productive discussion.

Although all interviewees believed that the determination of spatial thresholds is species-specific, there was also a general consensus on some of the spatial thresholds. For example, five out of six interviewees strongly agreed that an edge effect of $50 \mathrm{~m}$ around a patch of indigenous vegetation is essential in the process of spatial planning for biodiversity conservation in order to avoid edge effects. Edge effects increase as a result of increase in edge density. Edge density depends strongly on patch size and shape complexity per se (q.v. Table 2.1). In addition, the available empirical information shows that landscape fragmentation increases edge density (Andren, 1994). Edge density can accordingly affect urban biodiversity as a whole. As edge density increases, the patch interaction with its surroundings increases either positively or negatively (Dramstad et al., 1996; q.v. Table 2.1; Section 2.4.5.3). Observations made in the course of this research suggest that edge areas of patches of vegetation in the Wellington urban landscape are exposed to (1) extreme weather events, (2) high predator pressures, and (3) negative effects of human activities (q.v. Chapter 7).

\footnotetext{
${ }^{43}$ No quantitative thresholds have been defined for land cover heterogeneity, land surface perviousness, and landform diversity by Meurk and Hall (2006) (q.v. Table 3.6).
} 
In a New Zealand context, Young and Mitchell (1984) and Davies-Colley et al. (2000) revealed that edge effects are considerably reduced within ca. $50 \mathrm{~m}$ and ca. $40 \mathrm{~m}$ from the patch boundary, respectively. This is in agreement with similar research in urban New Zealand (Meurk and Swaffiled, 2000; Meurk and Hall, 2006; Meurk et al., 2016). This threshold is also consistent with international research (Murica, 1995). In Australia, a 50 m edge width was recommended to design buffer zones for highly vulnerable koala (Phascolarctos cinereus) (Port Stephens Council, 2001). As wind and sun exposure widen edge effects (Dramstad et al., 1996), it is not correct to assume that the width of the edge effect remains the same around a patch. In addition, edge areas are extremely susceptible to predator pressure in New Zealand because the presence of predators are thought to be higher along edges (Dijak and Thompson, 2000; Storch et al., 2005; Barbaro et al., 2012). Despite this, an edge depth of $50 \mathrm{~m}$ is thought to be acceptable in urban New Zealand, and was confirmed in this study, to safeguard the relevant keystone species from the ill-effects of climate change (Figure 3.3; q.v. Section 7.3.1).

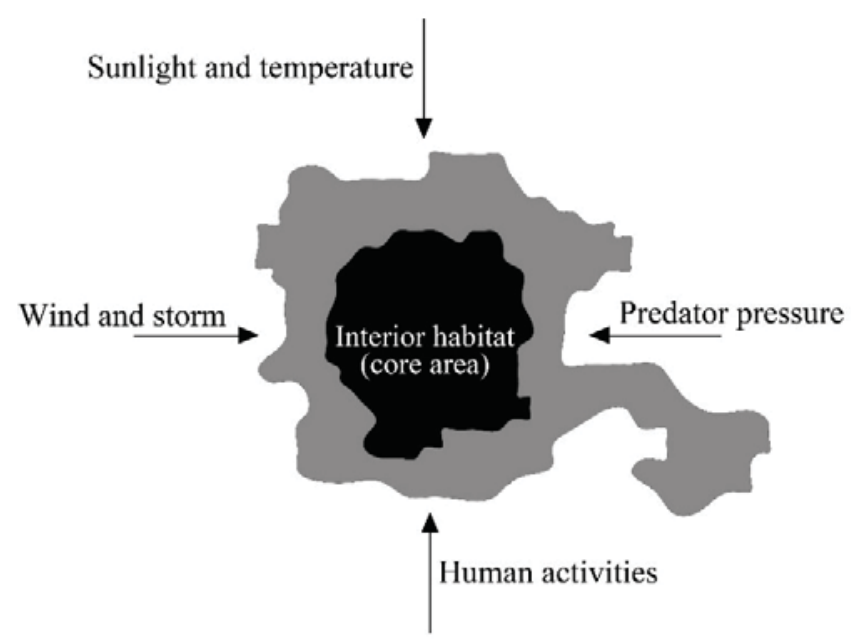

Figure 3.3. Examples of edge effects along edges of habitat patches.

No specific quantitative information was provided by the interviewees about other components of landscape pattern. Qualitative statements were given to support larger patch size, higher land cover heterogeneity and land surface perviousness, greater connectivity and proximity, and more complex landform diversity.

\subsubsection{Food availability and pest/weed control}

Irrespective of the importance of applying a spatial perspective for the design and management of patches of vegetation in a way that provides keystone species with primary habitats 
and stepping stones, the interviewees also underscored the key role of pest/weed control. They argued that without this, effective conservation of indigenous biodiversity in urban New Zealand would be unlikely. This concern can be strongly linked to the findings in current literature (Brown, 1997; Gillies and Clout, 2003; van Heezik et al., 2008 b; Innes et al., 2012; van Heezik et al., 2010; Aguilar et al., 2015; Goldson et al., 2015; Russell et al., 2015). Given this, the importance of pest-free urban wildlife sanctuaries was confirmed by all interviewees ${ }^{44}$. In addition, interviewees also emphasised that food availability is another important factor affecting urban biodiversity in urban New Zealand. For example, one of the interviewees stated that:

Plants are at the base of food webs and provide physical definition of habitat. For this reason, I consider them keystone [species]... Podocarps [e.g. rimu, matai, totara] are a biologically distinctive and iconic feature of New Zealand forests. As large trees they provide physical structure that helps define the forest and provides habitat to a wide range of animals, including fruit for birds and physical conditions for understorey plant species as well as sites of attachment for epiphytic plants.

Although food webs and pest/weed control are not spatial factors and seem to be less relevant to the most important components of landscape patterns, discussions with interviewees provided an in-depth picture of the crucial relationship between these non-spatial factors and the most important components of landscape pattern and immediate implications they may have for spatial design of land cover patterns, particularly in urban New Zealand (Table 3.7).

\subsubsection{The role of residential areas in biodiversity conservation}

The key role of residential areas in biodiversity conservation in urban New Zealand was also highlighted by the interviewees. People can contribute to biodiversity through creating biodiverse green roofs, backyards, and constructed wetlands in residential areas (Table 3.8). If designed and managed appropriately, these can serve as supplementary elements for biodiversity conservation in urban New Zealand. In an era of climate change, however, it should not be assumed that utilising such designed elements would alone be an effective response to extreme weather events.

${ }^{44}$ This issue is discussed in Chapter 8 in detail. 
Table 3.7

The role of non-spatial factors affecting fauna in urban New Zealand and implications for spatial design of land cover pattern interpreted from interviews.

\begin{tabular}{ll}
\hline Non-spatial factors & Implications for spatial design of land cover patterns \\
\hline Food availability & $\begin{array}{l}\text { Land cover heterogeneity should be as high as possible to provide species with different } \\
\text { sources of food year-round. }\end{array}$
\end{tabular}

In the Southern Hemisphere, south-facing slopes are critically important (as shelter or buffer against increased drought perhaps) and at the same time north-facing slopes may provide earlier flowering and fruiting. Therefore, landform diversity is particularly important to biodiversity conservation.

The percentage of indigenousness should be increased in a way that provides sustainable food sources for indigenous fauna. This can be achieved through natural regeneration mechanisms.

Pest/weed control

Lower edge density may help to protect against weed invasion because typically weeds benefit from the light along edges. Moreover, intensified winds may penetrate into wildlife habitats and this, accordingly, affects habitat climatic-environmental stability through influencing variables such as temperature and humidity. Lower edge density decreases the risk of predator-prey interface along edges.

Fencing is necessary for patches of ecological significance to help safeguard some vulnerable fauna species (e.g. little-spotted kiwi and tuatara) from the risk of predator pressures.

Table 3.8

The role of residential areas in biodiversity conservation in urban New Zealand.

\begin{tabular}{ll}
\hline Elements in the built-up areas & Rationale \\
\hline Green roofs & $\begin{array}{l}\text { Green roofs on high-rise buildings can be used by some vulnerable avifauna } \\
\text { as predator free refugia. If constructed in appropriate locations, green roofs } \\
\text { can also serve as stepping stones. }\end{array}$ \\
Backyards & $\begin{array}{l}\text { Backyards can be used by reptiles as primary habitats. If constructed in } \\
\text { appropriate locations, they can serve as stepping stones for avifauna and } \\
\text { provide them with food sources. }\end{array}$ \\
Constructed wetlands & $\begin{array}{l}\text { Constructed wetlands can be used by reptiles as primary habitats. If } \\
\text { constructed in appropriate locations, they can serve as stepping stones for } \\
\text { avifauna and provide them with food sources. }\end{array}$ \\
\hline
\end{tabular}

\subsection{CONCLUSION}

Study II achieved the four aims defined in advance of the research (q.v. Section 3.1). This Study highlighted some important issues that are not currently discussed or defined in the literature. Lessons learned and new findings from the results of Study II, can be summarised as follows:

First, taking the importance of indigenousness into particular consideration as a base for providing indigenous fauna with primary habitats, stepping stones, and sustainable food sources, this 
study reveals that the results derived from Study I are, to a large extent, relevant to urban New Zealand. Therefore, rankings provided by the international researchers, more specifically those provided by researchers and scientists in the Southern Hemisphere and Oceania, can also be used as input data for biodiversity research in urban New Zealand. For example, weights dedicated to the components of landscape pattern can be applied as a basis for multi-criteria suitability analysis of wildlife habitats in New Zealand urban landscapes to inform policymakers of suitable places (considering both space and time) for the establishment of new urban wildlife sanctuaries (q.v. Chapter 8).

Second, of the provided list of keystone species, some species are culturally important, while others are more ecologically significant. There is, however, a consensus on the most ecologically important avifauna (viz. bellbird, hihi, kererū, and tūī) and reptiles (viz. forest gecko and Wellington green gecko), which can be considered keystone species in the Wellington urban landscape.

Third, this study confirms that New Zealand urban biodiversity researchers are mostly concerned about the negative impacts of storm/wind events and rising temperatures as the most detrimental impacts of climate change on the keystone species and their habitats in the coming decades. This is in agreement with the available, but insufficient, empirical evidence in the literature. This concern led to the decision to undertake further in-depth spatial analysis of land cover patterns in the study area in relation to spatial requirements of the keystone species in the face of the aforementioned climate change impacts (q.v. Chapter 7).

Fourth, Study II also reveals that New Zealand urban biodiversity researchers strongly believe that pest-free urban wildlife sanctuaries could be the most effective response to the vulnerability of indigenous fauna that will be affected by predation by exotic mammals, many of which will benefit from rising temperatures. Although not considered as keystone species due to factors discussed earlier, culturally important species such as tuatara and little-spotted kiwi are believed to be potential ecologically proactive and influential keystone species, if urban New Zealand can provide suitable ecological conditions for them to become more widespread, and if their populations become stable (q.v. Chapter 8).

Fifth, the role of people in the built-up area was strongly highlighted in the course of the interviews. The interviewees believed that people can play a positive role in supporting biodiversity in New Zealand cities. For example, strategically planted and managed very small patches of vegetation in built-up areas are likely to provide additional food sources when enough food is not available. 
These micro-habitats can also potentially function as stepping stones to facilitate movement between fragmented patches of vegetation. Furthermore, small patches of vegetation in built-up areas can serve as primary habitats for reptiles.

Sixth, with reference to the spatial ecologies of the selected species, spatial planning for bellbird, hihi, kererū, and tūī can be conducted at landscape and class levels (q.v. Chapters 6 and 7), while urban wildlife habitats for forest gecko and Wellington green gecko should be managed at the patch level (q.v. Chapter 8). The little penguin is a unique species and is completely confined to small areas in coastal zones. As the long-tailed bat is unlikely to be easily reintroduced to the Wellington urban landscape, this species can be removed from the list of keystone species. This species, however, could be reintroduced in the coming decades provided that large patches of indigenous forest are effectively managed for this specific purpose. If the study area provides suitable conditions for vulnerable species such as tuatara and short-spotted kiwi, it can also serve as a potentially suitable habitat for a wide range of other vulnerable indigenous species such as kākā, rifleman, and takahē. These were suggested as potential additional keystone species by some of the interviewees. Due to the lack of sufficient empirical evidence in the literature, however, they are not regarded as keystone species for the purposes of this research.

Seventh, landscape restoration through natural regeneration mechanisms is vital to increase land cover heterogeneity in the study area. In particular, the two classes of Fernland and Manuka and Kanuka can potentially be regenerated in the study area. This has been confirmed based upon direct observations made by the interviewees in several places close to the study area (q.v. Chapter 6).

Eighth, it is not necessary to quantify and measure edge contrast in this research, because this factor probably has the least impact on the selected keystone species. Despite this, initial information was presented to provide a basis for future research (q.v. Tables 3.4 and 3.5).

Ninth, according to information generated in this study, the spatial thresholds derived from the literature are highly site- and species-specific (q.v. Table 3.6). Despite this, there is a general consensus on an edge depth of $50 \mathrm{~m}$ in order to apply for the identification and measurement of edge density in relation to patch size and shape complexity in urban New Zealand. This can be considered as a strong indicator for landscape pattern analysis and interpretation (q.v. Chapters 7 and 8).

Overall, information gained from Study II can be used, with a high degree of certainty, as input data for Study III (q.v. Chapters 4-8; cf. Figure 1.10). 


\section{CHAPTER 4}

Study III: Measuring the spatial extent of land cover classes: indigenous vs. exotic

\subsection{RESEARCH AIMS}

In Study I, indigenousness was identified and ranked by international researchers as one of the most important components of landscape pattern affecting the presence, richness, and abundance of fauna in urban landscapes (q.v. Chapter 2). In Study II, the importance of indigenousness was underscored by New Zealand subject-matter experts as the single most irreplaceable component of landscape pattern affecting the overall level of biodiversity in New Zealand (q.v. Chapter 3). Aside from this, there is an obligation to support the preservation of indigenous wildlife in New Zealand (q.v. Chapter 1), in particular according to Te Tiriti ${ }^{45}$. The main aims of this chapter are to:

(1) Provide a spatial picture of the current status of indigenous vs. exotic flora in terms of indigenousness, and accordingly, land cover heterogeneity, and land surface perviousness in the study area - Wellington urban landscape (q.v. Figure 1.6) and its implications for landscape architecture and land use planning practices;

(2) Provide a deeper understanding of the key role of indigenous flora in the continuity of biodiversity in urban New Zealand in general and in the study area in particular;

(3) Address the ecological role of some exotic flora in support of indigenous biodiversity in urban New Zealand.

${ }^{45}$ Te Tiriti or the Treaty of Waitangi is an agreement between British Crown and Māori chiefs signed on 6 February 1840 in New Zealand. 


\subsection{THE IMPORTANCE OF INDIGENOUS FLORA IN NEW ZEALAND}

According to Brockie (2007), about $80 \%$ of the 2,500 species of indigenous conifers, flowering plants and ferns are endemic to New Zealand. In addition, 71\% of birds breeding in New Zealand before human arrival have been recognised as endemic species (cf. Buller, 1888; Wilson, 2004). The introduction of a large number of exotic flora and fauna to the country has deeply affected New Zealand indigenous biodiversity (Allen and Lee, 2006; q.v. Chapter 1). Since the early 1800s, Europeans have imported a wide range of exotic flora and fauna to New Zealand (Dawson, 2010). In addition, the modification of land by humans has widely affected indigenous landscapes (Walker et al., 2006; 2008). Approximately 70\% of the New Zealand land environment has been modified by humans causing the extinction of 32\% of indigenous land and freshwater birds, $18 \%$ of endemic seabirds, 3 of 7 species of frogs, and at least 12 invertebrates (Statistics New Zealand, 2002; cf. Holdaway, 1999; Duncan and Young, 2000; Duncan and Blackburn, 2004; Collins et al., 2014; Perry et al., 2014). The first group of people to settle in New Zealand ca. the 1300s, were Polynesians, now known as Māori and Moriori. Although somewhat controversial, their hunting practices, the introduction of certain commensal animals (such as kurī, the now extinct in New Zealand, Polynesian dog Canis familiaris; and kiore, the Pacific or Polynesian rat Rattus exulans) and anthropogenic caused ecosystem degradation (particularly clearing by fire of almost half of all lowland and montane forest) is thought to have given rise to the extinction of 34 endemic land birds and several endemic herpetofauna or at least significant declines in their populations (McGlone 1989; Towns and Daugherty, 1994; Ministry for the Environment, 1997 cited in Craig et al., 2000). The removal of indigenous land cover and extinctions or population pressures on indigenous species continued, with different causes and results, from the late 1700s when the first Europeans arrived in New Zealand ${ }^{46}$.

Before human arrival, 85-90\% of New Zealand was covered by indigenous forests (McWethy et. al. 2010). By 1800, forest cover had been reduced to approximately 70\% of the land (Ewers et. al. 2006). By the 1850s, when European settlement was becoming widespread, more than $40 \%$ of pre-human forest cover (from ca. 3000 years before now) had been removed due to the combined impacts of both Polynesian (mostly in the period 650 to 850 years ago) and European people (from the mid-1800s until now) and, to a lesser and more controversial extent, the impact of some non-human causes such as periodic climatic changes, major natural wildfire events, and volcanic eruptions (McGlone 1983; 1989; McWethy et. al., 2010). Between 1847 and 1909, Europeans destroyed over 60\% of the remaining forest (ca. 4.5 million ha) (McGlone 1983) and

${ }^{46}$ Māori populations and their activities on land had some impacts on ecosystems and biodiversity but it was less profound than what happened rapidly when Europeans arrived. 
negative impacts on ecosystems due to erosion, nutrient transport, and eutrophication were probably more severe than the earlier impacts of Māori forest burning (Pawson and Brooking, 2002; McWethy et. al., 2010).

Europeans were also responsible for the introduction of significant numbers of exotic species of both flora and fauna, some of which have major ongoing negative impacts on indigenous flora and fauna (Duncan and Blackburn, 2004; q.v. Section 1.1.5). Today, although three quarters of all remaining forest is protected from future clearance and regeneration efforts are being made, approximately $75 \%$ of original forest cover has been destroyed and substantial numbers of New Zealand flora and fauna are classified as threatened species (Wilson and Given, 1989; Ewers et. al. 2006); research shows that since human arrival in New Zealand, at least 6 plants have become extinct, and 184 are now nationally threatened including 94 nationally critical taxa, 46 nationally threatened taxa, and 44 nationally vulnerable taxa (De Lange et al., 2010). Mark (2010) notes that in addition to the aforementioned figures, 651 taxa are also classified as at risk encompassing: declining, 83; recovering, 6; relict, 20; and naturally uncommon, 542.

While New Zealand indigenous biodiversity is currently under pressure from exotic species already introduced to the country, the situation may become more complicated in the coming decades because of increased predator pressure caused, in part, by the mast seeding phenomenon resulting from rising temperatures (q.v. Section 1.4.4).

According to Clarkson et al. (2007), only 1.96\% of current urban New Zealand is covered by the class "Indigenous Forest" that is indicative of mature indigenous land cover and thought to be the primary habitat for a wide range of indigenous fauna (q.v. Sections 1.1.5 and 3.3.1.1). The class Indigenous Forest provides indigenous fauna with primary habitat and food sources (q.v. Table 3.2). Indigenous fauna, in turn, play a key role in pollination and seed dispersal in support of indigenous flora (q.v. Sections 1.4.3 and 6.2.1). Thus, the loss of indigenous flora in urban landscapes may be regarded as a threat to the survival of indigenous fauna and vice versa. Despite this, some exotic flora also can support indigenous fauna, and this should not be set aside, particularly because New Zealand urban landscapes currently contain large expanses of exotic flora (q.v. Chapter 1). This means that it is necessary to define a balance between indigenous and exotic land cover classes in urban landscapes in order to maximise the support and provision of ecosystem services required both to support indigenous fauna and to maintain and/or improve the healthy functioning of ecosystems over time. 


\subsection{MATERIALS AND METHODS}

Research methods included a review of the current literature on the influence of indigenous and exotic flora on biodiversity followed by a landscape-level analysis of land cover patterns in the Wellington urban landscape. This was done to reveal the current status of indigenous and exotic land cover classes using the Land Cover Data Base v. 4.1 - LCDB (Landcare Research, 2015). LCDB is a multi-temporal and thematic classification of New Zealand's land cover. Regularly corrected, evolved, and updated since 1996 by different stakeholders including regional councils and the New Zealand Department of Conservation, version v.4.1 of the dataset has been created through the automatic detection of spectral changes based upon SPOT 5 satellite imagery (October 2011 - February 2013), and improved using 2012 New Zealand's Kyoto Land Use Mapping and spectral methods verified with radar analysis (ALOS PALSAR). This version was built for polygon typology and released in July 2015 (Landcare Research, 2015; Dymond et al., 2017). For data augmentation, ground-truthing ${ }^{47}$ was also conducted to make sure that the accuracy of spatial data is acceptable, more specifically in areas where the accuracy of spatial data was not reliable or an update was required (cf. Wellington City Council Open Data, 2015).

First, irrespective of information obtained in Study II (q.v. Chapter 3), scholarly publications containing empirical data were reviewed to identify how indigenous flora may support urban fauna (q.v. Section 4.4). The review also included consideration of both the advantages and the disadvantages of utilising exotic flora in urban landscapes in a way that supports biodiversity. The published data in the current literature was collected from peer-reviewed papers indexed in online scientific databases (e.g. Scopus and ISI Web of Knowledge). While international examples were studied, particular heed was given to research from New Zealand to provide a more focused and detailed picture of the available information.

Second, a landscape-level spatial analysis of the Wellington urban landscape was undertaken to identify and depict the extent of the current land cover classes and quantify and measure the degree of indigenousness, land cover heterogeneity, and land surface perviousness in terms of land cover classes currently present in the study area (q.v. Section 4.5). LCDB was used as a platform for generating an informative and measurable land cover map of the study area in the GIS environment. Appropriate analysis tools including Extract and Overlay were utilised to generate the map (Figure 4.1). A layer was dedicated to each land cover class. To do this, classes were differentiated from each

${ }^{47}$ Informal and casual observations were made by the researcher, specifically in some coastal areas in Island Bay to check the accuracy of land cover data. No significant mismatches were observed between the spatial dataset and the real environment. 
other using the attribute table of LCDB. Next, each class was illustrated in the GIS environment using the standard colour codes suggested by Landcare Research (2015). The tool Dissolve from Data Management Tools was employed to condense patches of the same class. Spatial Statistics Tools were then used to calculate the total area of each land cover class in the study area. Conversion tools were also used to convert the format of the generated map from polygon to raster and vice versa depending on the requirement of the spatial analysis. The geographic coordinate system used to analyse the map was GCS WGS 1984. Standard definitions of the land cover classes are available in Appendix V.

\subsection{AN OVERVIEW OF THE EFFECTS OF FLORA ON FAUNA}

Forman (1995) argues that indigenous flora is so important for maintaining biodiversity that even small patches of indigenous vegetation in otherwise modified landscapes (e.g. urban landscapes) should be actively protected (cf. Forman and Godron, 1986; Turner, 1989; q.v. Table 2.1). Some researchers have established a connection between indigenous vegetation and overall levels of biodiversity worldwide (Fischer et al., 2009). This means that a greater proportion of land covered by indigenous flora may be positively correlated with greater biodiversity. Empirical research suggests that when a landscape consists of coverage of more than 30\% indigenous flora, the adverse effects of landscape fragmentation on wildlife species will be reduced to an acceptable level ${ }^{48}$ (Andren, 1994). The presence, richness, and abundance of fauna can be linked to the extent of flora in urban landscapes, worldwide (Cornelis and Hermy, 2004; Sandstrom et al., 2006; Pacheco and Vasconcelos, 2007; Smith, 2007; Khera et al., 2009; Garden et al., 2010; Villegas and GaritanoZavala, 2010; de Toledo et al., 2012; Chong et al., 2014; Ferenc et al., 2014; Paker et al., 2014; Sanz and Caula, 2015; Threlfall et al., 2016; Jasmani et al., 2017). Similar results have been observed in urban New Zealand (van Heezik et al., 2008 a; MacLeod et al., 2012; van Heezik et al., 2013). In particular, a study of twenty-two cities across the world, including five cities in the Southern Hemisphere (viz. Adelaide, Auckland, Durban, Melbourne, and Singapore), indicated that the percentage of indigenous vegetation in urban landscapes is negatively correlated with the extinction rate of indigenous plants (Hahs et al., 2009) that are used by indigenous fauna as an alternative source of food.

Recently, Aronson et al. (2014) studied more than one-hundred cities worldwide including three cities in New Zealand (viz. Auckland, Dunedin, and Hamilton) and found that indigenous vegetation supports higher concentrations of both indigenous and exotic bird and plant species.

${ }^{48}$ This is where minimum requirements for the survival of wildlife species are met. 
Increasing the percentage of indigenous land cover in New Zealand modified landscapes has been proposed to decrease the bio-security risks imposed by some exotic flora and fauna including biological invasions (Meurk and Swaffield, 2000; 2007; q.v. Table 3.2). According to Meurk (1999), at least $20 \%$ of the total land area is required to be covered by indigenous land cover classes to ensure the survival of indigenous biodiversity in urban New Zealand (q.v. Table 3.6).

The overall results of empirical research conducted by New Zealand researchers are testimony to the fact that indigenous fauna is extremely dependent on indigenous flora. For example, the mutual ecological relationship between lizards, skinks and some indigenous plants has been observed and proved $^{49}$ (Whitaker, 1987; Wotton, 2002). Clout and Gaze (1984) show a key ecological connection between indigenous forests and indigenous avifauna.

A four-year (1993-97) study of endemic long-tailed bats in the Lower Eglington Valley in Fiordland National Park in the South Island found that very mature (ca. 100 years to $>600$ years of age) indigenous trees are actively chosen by bats for roosting. In addition, New Zealand indigenous invertebrates require indigenous plants to survive (Patrick, 1994). A field survey of 195 locations in the North Island of New Zealand revealed that the presence of indigenous avifauna, including kererū and tūî, depends widely on the percentage of indigenous forest cover and intensive pest control. In particular, the study found that in areas where the forest cover is < ca. 5-10\% of the total landscape, indigenous forest cover is more important to avifauna than pest control (Ruffell and Didham, 2017). What this means for landscape architecture research and practice is that it is vital that the percentage of indigenous land cover in urban landscapes is increased in order to support indigenous fauna.

The keystone fauna species initially listed based upon the literature review (q.v. Chapter 1), and revised in the course of Study II (q.v. Chapter 3), depend profoundly on the land cover class Indigenous Forest for the provision of habitat and food (Craig et al., 1981; Stewart and Craig, 1985; Clout, 1990; Castro et al., 2003; Dilks, 2004; Thorsen et al., 2004; Heather and Robertson, 2005; Sagar and Scofield, 2006; Emeny et al., 2009; Powlesland et al., 2011; Spurr et al., 2011; Makan et al., 2014; Robertson et al., 2017; q.v. Chapters 1 and 3).

As touched upon, there is also worldwide evidence that some exotic flora can be advantageous to the conservation of indigenous biodiversity. For example, some researchers including Fischer et al. (2009) and Szlavecz et al. (2011) discuss how exotic flora can play a

\footnotetext{
${ }^{49}$ While indigenous plants provide primary habitats and food sources for these reptiles, they contribute to the process of seed dispersal and pollination in order for plants to regenerate or colonise in other places (q.v. Chapter 1).
} 
compensatory role in supporting indigenous fauna in the absence of intact indigenousness. Moreover, the findings of some studies reveal that indigenous avifauna may depend on exotic plants to survive in urban landscapes (Corlett, 2005). In New Zealand, studies have also been undertaken investigating the significance of exotic flora in the support of indigenous fauna. A review conducted by Pawson et al. (2010) provided details of the relationship between exotic flora and indigenous fauna, specifically threatened species, and confirmed that a considerable amount of New Zealand indigenous fauna may obtain benefits from exotic flora in various ways. Research in Hamilton reveals that some exotic flora species may be utilised by the long-tailed bat in winter (Borkin and Parsons, 2011; Dekrout et al., 2014). Similarly, Gray and van Heezik’s (2016) research in Dunedin shows some exotic trees such as sycamore (Acer pseudoplatanus) and oak (Quercus robur) supply foraging sources for indigenous avifauna, more specifically during winter. Evidence on the role of exotic flora in providing kererū, tūī, and bellbird with food sources, when and where indigenous flora are absent or insufficient, has also been documented (q.v. Chapters 1 and 3).

\subsection{RESULTS}

Figure 4.1 provides a detailed picture of the extent of land cover classes distributed in the Wellington urban landscape. Of the thirty-three land cover classes defined as depicting the New Zealand environment (Landcare Research, 2015), the Wellington urban landscape consists of sixteen classes (Table 4.1).

Currently, about 39.93\% of the total study area has been built on, and the remainder of the land is covered by a wide range of green and open spaces (q.v. Figure 4.1 and Table 4.1). Green space per capita in the Wellington urban landscape is very high (ca. $400 \mathrm{~m}^{2}$ per capita). Out of sixteen land cover classes present in the study area, only four are indigenous, namely Broadleaved Indigenous Hardwoods (22.98\%), Indigenous Forest (0.9\%), Matagouri or Grey Scrub (0.22\%), and Herbaceous Freshwater Vegetation (<0.02\%). Amongst them, only Indigenous Forest $(0.9 \%)$ is a mature indigenous land cover class capable of providing indigenous fauna with primary habitats, food sources, and thereby ensuring indigenous biodiversity and high-quality ecosystem services (cf. Section 3.3.1.1). Moreover, five exotic land cover classes were identified in the study area, namely Gorse and/or Broom (12.53\%), High Producing Exotic Grassland (11.06\%), Exotic Forest (6.23\%), Urban Parkland and/or Open Space (3.02\%), and Low Producing Grassland (2.55\%) (Figure 4.2). Table 4.1 provides key information about the rate of indigenousness $(n=4,24.12 \%)$, land cover heterogeneity $(\mathrm{n}=16)$, and land surface perviousness $(61.14 \%)$. 
Table 4.1

Land cover classes present in the study area.

\begin{tabular}{lrrr}
\hline Class Name & z Area (ha) & Total land area (\%) & Indigenous \\
\hline Built-Up Area & 5087.5 & 37.65 & Yes \\
Broadleaved Indigenous Hardwoods & 3105.5 & 22.98 & 12.53 \\
Gorse and/or Broom & 1693.3 & 11.06 & \\
High Producing Exotic Grassland & 1495.1 & 6.23 & 3.02 \\
Exotic Forest & 842.7 & 2.55 & Yes \\
Urban Parkland/Open Space & 408.2 & 1.21 & Yes \\
Low Producing Grassland & 345.1 & 1.07 & \\
Transport Infrastructure & 163.9 & 0.90 & 0.48 \\
Surface Mine or Dump & 145.5 & 0.22 & \\
Indigenous Forest & 121.7 & $<0.04$ & $<0.02$ \\
Sand or Gravel & 65.3 & $<0.02$ & Yes \\
Matagouri or Grey Scrub & 30.4 & 100 & \\
Lake or Pond & 3.7 & 1.3 & \\
Forest Harvested & 1.7 & & \\
Gravel or Rock & 0.4 & & \\
Herbaceous Freshwater Vegetation & 13511.3 & & \\
Total area & & & \\
\hline
\end{tabular}

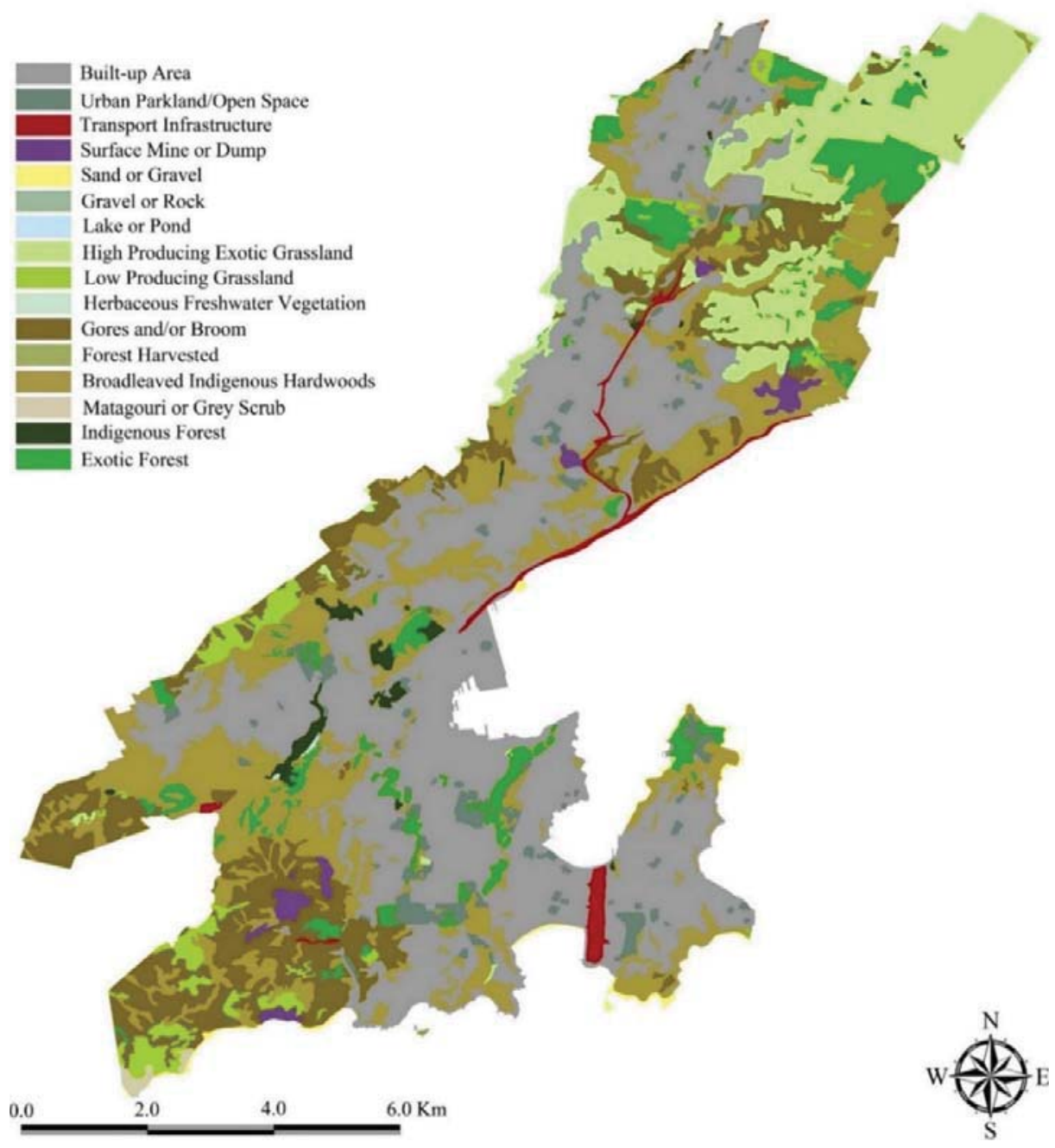

Figure 4.1. Land cover classes present in the study area - created based on LCDB (Landcare Research, 2015). 

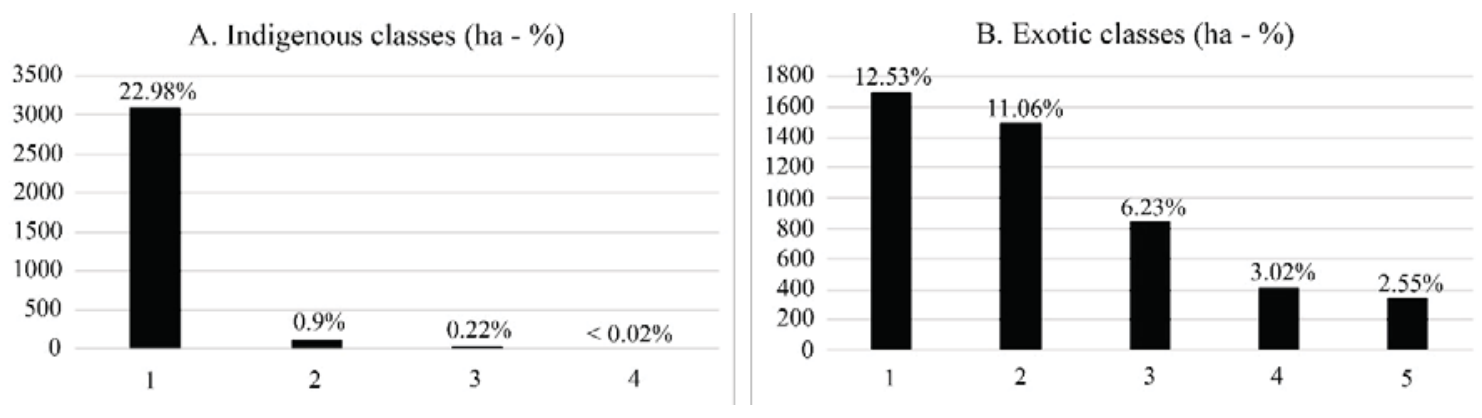

Figure 4.2. Indigenous and exotic land cover classes present in the study area (cf. Table 4.1).

A: Indigenous classes: Broadleaved Indigenous Hardwoods (1), Indigenous Forest (2), Matagouri or Grey Scrub (3), Herbaceous Freshwater Vegetation (4).

B: Exotic classes: Gorse and/or Broom (1), High Producing Exotic Grassland (2), Exotic Forest (3), Urban Parkland/Open Space (4), Low Producing Grassland (5).

\subsection{DISCUSSION}

\subsubsection{The importance of scale and resolution}

Spatial resolution and the availability of a detailed land cover classification system play a key role in identifying and plotting the land cover classes present in the area under study. The spatial resolution of data needs to be sufficiently fine-grained to reveal detailed ecological information about a wide range of land cover classes. Thus, a detailed land cover classification system is required to address urban biodiversity, provide opportunities to analyse urban landscapes, and ultimately, reveal issues that are unlikely to be easily diagnosed and understood through using typical maps (cf. Freeman and Buck, 2003). Using LCDB, it was calculated, for example, that only 121.7 ha (0.9\%) of the total study area of 13511.3 ha consists of the class Indigenous Forest that is indicative of a mature indigenous land cover class in the study area representing intact natural patches of vegetation before the arrival of humans (q.v. Sections 1.1 .5 and 6.4.1). Therefore, the individual share of each Wellington resident of the existing indigenous forests is only about $6 \mathrm{~m}^{2}$ (cf. ca. $400 \mathrm{~m}^{2}$, as greenspace per capita). This difference is alarming, reinforcing the need for landscape architecture researchers and practitioners to employ detailed land cover classification systems when addressing urban biodiversity issues so that misconceptions concerning urban green spaces are avoided. This is important because not all green spaces are capable of supporting biodiversity (q.v. Chapter 3). This difference, reemphasises the importance of scale (Cushman et al., 2008) and the key role of detailed maps in biodiversity research (Botequilha Leitao et al., 2006). Decisions regarding choosing systems by which land cover is classified (i.e. the spatial scale and resolution) also affect the degree of land cover heterogeneity. As shown, land cover heterogeneity in the study area is relatively high ( $\mathrm{n}=16)$. 


\subsubsection{Opportunities for ecological succession}

Although land surface perviousness in the study area is high (i.e. 61.14\% of the study area is covered by various patches of vegetation and/or non-built-up areas), a high proportion of land is covered by exotic land cover classes (q.v. Table 4.1). Thus, to compensate for the lack of intact indigenous land cover, landscape restoration practices through natural (or if plausible managed) regeneration may be a practical option to increase the percentage of areas covered by indigenous flora (q.v. Chapter 6). These opportunities can be discussed when the concept of ecological succession is understood. Ecological succession is a process whereby one ecosystem (i.e. land cover class) is gradually transformed into another.

According to Landcare Research (2015), left undisturbed, patches of vegetation covered by gorse (Ulex europaeus) and/or broom (Cytisus scoparius) can be converted into the class Broadleaved Indigenous Hardwoods and these, accordingly, can be transitioned toward the class Indigenous Forest over time. There is ample evidence to show that areas covered by the class Gorse and/or Broom are very likely to be replaced by indigenous flora under suitable circumstances (Williams, 2011; q.v. Chapter 6). Further observations made by Meurk and Swaffield (2000) suggest that some indigenous shade-tolerant trees such as tōtara (Podocarpus totara), mataī (Prumnopitys taxifolia), and kahikatea (Dacrycarpus dacrydioides) can be established under canopies of exotic species Douglas fir (Pseudotsuga) and pine (Pinus spp) (Ogden et al., 1997). These plant species are all present in the study area (Dymond and Shepherd, 2004).

Currently, $12.53 \%$ and $22.98 \%$ of the Wellington urban landscape are respectively covered by the classes Gorse and/or Broom and Broadleaved Indigenous Hardwoods, providing opportunities for natural regeneration through ecological succession. Given that at least 21,400 new dwellings need to be constructed in urban Wellington by the year 2043 in order to respond to the projected urban population growth (Wellington City Council, 2015), identifying the spatial dispersal of the potential land cover classes capable of transition towards the class Indigenous Forest holds the key to avoiding misallocation of land (from a biodiversity perspective) to future housing and infrastructure development. For example, allocation of lands covered by the class Gorse and/or Broom to urban development (e.g. new houses and land associated with essential infrastructure such as transportation facilities and power lines) may deprive the study area of potential natural regeneration opportunities through ecological succession over time. 


\subsubsection{Compensatory roles of exotic flora}

In urban landscapes that already contain non-native flora, some exotic flora may play a compensatory role in supporting indigenous fauna. Therefore, it is necessary to recognise the possible benefits of exotic flora. For example, patches of exotic vegetation can be regarded as stepping stones in order to facilitate species' movement between patches of indigenous vegetation (q.v. Chapter 3). Meurk and Hall (2006) suggest that the distances between patches of indigenous vegetation should be defined based upon the size of patches. Conducting modelling research in Auckland, Christchurch and Queenstown, they argue that the distances between patches $>6.25$ ha, $>1.56$ ha, and $>0.1$ are required to be $<5000 \mathrm{~m}$, between 1000 and 2000m, and $<200 \mathrm{~m}$, respectively. Given that these spatial thresholds may serve to meet the minimum ecological requirements for New Zealand urban biodiversity negatively affected by humans and their introduced flora and fauna over the past centuries, in the absence of enough connectivity and proximity between patches of indigenous vegetation in the study area, land covered by exotic flora may serve as stepping stones to reduce the existing distances and facilitate the movement of urban fauna. This can be regarded as a new opportunity for the flow of genes and dispersal of seeds. This type of spatial connectivity, however, may concurrently increase the risk of pest and weed dispersal from one patch to another (Sullivan et al., 2009). Therefore, debate on spatial connectivity remains an area of conflict in urban biodiversity research (Barnes, 2000; Botequilha Leitao et al., 2006, passim; q.v. Section 2.4.5.2), more specifically in urban New Zealand where the presence of pests and weeds is an obvious challenge (Williams and Timmins, 2002; q.v. Sections 1.1.5 and 3.3.2).

Exotic flora may also be utilised as a barrier to edge effects (Murcia, 1995) such as air and noise pollution, intensified windstorms, and sunlight penetration into the patch. In the absence of indigenous flora, patches of exotic vegetation may be employed as a buffer to alleviate edge effects (Fischer et al., 2009). In New Zealand, empirical research shows that exotic Pinus radiata (widely grown for timber in plantations in New Zealand) may be suitable for this purpose (Brockerhoff et al., 2008).

Research in New Zealand demonstrates that penetration of light, temperature and wind into urban wildlife habitats may be controlled by some exotic plants. Denyer et al. (2006), for instance, conducted experimental research in the Waikato and revealed that Pinus radiata (known in New Zealand as 'pine') plantations can effectively buffer edge effects at edge areas of indigenous forests. The main serious concern, however, is that juxtaposition of indigenous and exotic flora may increase the risk of weed and pest dispersal into patches of indigenous vegetation. For example, gorse and 
broom are aggressive weed plants that benefit a number of introduced mammals (Otago Regional Council, undated), which are major threats to indigenous biodiversity in New Zealand (MacCarter and Gaynor, 1980). Gorse and broom seeds may remain viable in the soil for up to 80 years (q.v. Chapter 6). Pine can also become a weed species of great concern in certain ecosystems. On the other hand, the presence of exotics means that New Zealand indigenous avifauna may be better able to overcome food source scarcity during winters depending on the exact species available (Gray and van Heezik, 2016).

Regardless of the size and extensiveness of patches of exotic vegetation, they can at times supply supplementary foraging sources when indigenous flora cannot do this effectively, due to scarcity in areas covered by the class Indigenous Forest. Indigenous avifauna feeding on nectar of exotic Eucalyptus species and other exotic flora were observed in Wellington urban landscape parklands and reserves for example from 2015 to 2018 by the researcher.

\subsection{CONCLUSION}

In this chapter, the study area was quantified and measured in terms of indigenousness, land cover heterogeneity, and land surface perviousness (q.v. Table 2.1). A detailed spatial picture of the status of indigenous and exotic flora was also provided though measuring the spatial distribution and extent of land cover classes currently present in the Wellington urban landscape. The research reveals three important implications for landscape architecture and land use planning practices as follows:

First, indigenous purity is unlikely to be achievable in the real world (cf. Chapter 3). In New Zealand and other Southern Hemisphere countries, the question of indigenous vs. exotic species (and thereby, landscape restoration practices) is one of the most topical, compared to North American and European countries. Information provided in this chapter does not advocate the planting of exotic species in urban landscapes, particularly those known to be invasive and have negative impacts on indigenous species in a New Zealand context. The aim instead has been to draw attention to potential ecological benefits of some exotic species that are already present in urban landscapes. Therefore, in line with efforts related to landscape restoration for increasing indigenousness in urban landscapes, the positive role of some non-invasive exotic plants in terms of supporting biodiversity should not be ignored. Allowing a considered proportion of non- or less-invasive exotic flora to exist in cities could be more practical than other alternatives such as eliminating all exotic plant species, particularly those that are believed to play a key role in ecological succession towards indigenous plant communities, or 
that provide food sources for indigenous faun ${ }^{50}$. In this chapter, the scarcity of land in New Zealand covered by the class Indigenous Forest was revealed in the study area. With regard to the key role of this class in supporting indigenous biodiversity, further research is required to suggest spatial strategies for maintaining and/or increasing the percentage of this class. In response to this need, possible opportunities are scrutinised in the following chapters (q.v. Chapters 5 and 6).

Second, due to the risk of weed and pest dispersal and the probable negative effects of some exotic plants on neighbouring indigenous habitats, exotic flora should be very cautiously maintained and/or established in urban landscapes. Specifically in the case of the study area, an in-depth insight into the role of indigenous and exotic plant species in urban landscapes could enable decision-makers to make appropriate decisions when allocating land to different purposes. For example, knowledge about ecological succession and the ability of some exotic species such as areas covered by the class Gorse and/or Broom to enable natural regeneration to the class Broadleaved Indigenous Hardwoods and next, to the class Indigenous Forest, may shed light on the process of dedicating land for future housing development in the study area. While a common belief is that gorse and broom are exotic and thereby useless, or even threatening, if managed appropriately, they may be considered as an opportunity for faster transition towards a range of indigenous land cover classes than 'bare' land. Chapter 6 will look at spatial potentials for landscape restoration through natural regeneration in detail using areas covered by the class Gorse and/or Broom (q.v. Chapter 6).

Third, recognising the compensatory role of some exotic flora in supporting indigenous biodiversity through providing indigenous fauna with stepping stones between habitat patches and additional food sources, can help landscape architects make distinctions between threats and opportunities for indigenous biodiversity associated with exotic flora in urban landscapes. This, accordingly, necessitates the importance of collaborating with scientists in other fields of research to maximise the advantages of establishing a balanced and spatially-appropriate proportion of indigenous to exotic flora in cities, and at the same time, to minimise any likely disadvantages of the presence of some exotic flora (cf. Chapters 2 and 3), such as those that are invasive or have negative impacts on indigenous species.

${ }^{50}$ Aside from this, the removal of all exotic plant species would be highly expensive, if not impractical. 


\section{CHAPTER 5}

Study III: Landform diversity and indigenousness: a key relationship

\subsection{RESEARCH AIMS}

In Study I, the importance of landform diversity was revealed and discussed, as one of the most important components of landscape pattern affecting urban biodiversity as a whole (q.v. Chapter 2). In Study II, landform diversity was identified as one of the most effective factors in spatial planning for biodiversity conservation in New Zealand (q.v. Chapter 3). The key role of landform diversity in the study area should be taken into particular consideration because Wellington is one of the world's most topographically diverse landscapes (q.v. Section 1.4). This shapes unique landscape characteristics for the study area. While the rarity of indigenous flora may negatively affect indigenous fauna in the Wellington urban landscape (q.v. Chapters 3 and 4), the diversity of slope aspects may be regarded as an opportunity to help safeguard indigenous fauna against extreme weather events in general, and rising temperatures in particular. Thus, Chapter 5 aims to:

(1) Examine the role of landform diversity as an important factor for quantifying and measuring the capability of urban landscapes in safeguarding vulnerable fauna against the negative effects of rising temperatures;

(2) Identify, quantify, and measure landform diversity in relation to indigenousness, in order to inform urban policy makers about where to keep such areas undisturbed in order for indigenous fauna to be able to temporarily migrate to south-facing slopes that are covered by the two classes Indigenous Forest and Broadleaved Indigenous Hardwoods in event of rising temperatures and/or heat waves.

\subsection{THE IMPORTANCE OF LANDFORM DIVERSITY}

In the New Zealand context, Landform diversity, among other components of landscape pattern (q.v. Chapter 2), is considered as one means to support biodiversity in the face of rising temperatures, as similarly confirmed by others in the Northern Hemisphere (Markham et al., 1993; 
Pernetta et al., 1994; Halpin, 1997; q.v. Chapter 2). In this context, landform diversity is defined as the diversity of elevations, slopes, and aspects that provide a wide range of micro- and macro-ecosystems and habitats for fauna in a given landscape (q.v. Table 2.1). It provides climate heterogeneity, and therefore may safeguard, or at least support, a greater number of species over time; this is due to the increased diversity in environmental variables such as temperature, moisture, and soil type in a heterogeneous topography (Fischer et al., 2009). Research in England (Rorison et al., 1986) and in the United States (Ackerly et al., 2010), for example, indicates that even at a fine scale, temperature differences between south- and north-facing slopes can be substantial, ranging from $8^{\circ} \mathrm{C}$ to $12^{\circ} \mathrm{C}$. Furthermore, amid the uncertainties and dynamics associated with climate change, landform diversity could play an important role in protecting biodiversity through creating diverse habitat conditions for different species in the face of different impacts that may be imposed by climate change. Patches of vegetation with diverse topography provide a spectrum of variable microclimatic conditions for a wide range of flora and fauna through the shading and cooling effects of valleys and land relief (Noss, 2001). Dobrowski et al. (2011) argue that in the Northern Hemisphere, positions such as north-facing slopes, valley bottoms, and steep canyons where long-term cool and moist conditions are maintained, are best for ensuring the continuation of biodiversity over time. Research in the United States indicates that some topographical features such as valleys, slopes, and aspects have a key role in maintaining biodiversity in a climate that is getting warmer (Olson et al., 2012).

In Southern Hemisphere countries, where indigenous biodiversity has been widely affected as a result of land cover change and ecosystem degradation, habitat loss and the introduction of invasive species (q.v. Section 1.1.4), south-facing slopes (i.e., those facing away from the sun) can be regarded as an opportunity for the possible establishment of strategic havens for urban fauna. If the spatial and environmental requirements related to other components of landscape pattern (e.g. patch size, connectivity and proximity, and shape complexity) are properly met, wildlife habitats protected against direct sunlight and solar radiation may be able to provide safe havens for those fauna that are more vulnerable to warmer conditions in the Southern Hemisphere (q.v. Table 1.5). Although landform diversity should not be seen as a panacea for biodiversity conservation questions in cities, this characteristic can be taken into consideration as a determinant, not as the single most important factor, in the process of planning for urban green infrastructure development (Gill et al., 2007; Tzoulas et al., 2007; Pauleit et al., 2011; Cameron et al., 2012; Andersson et al., 2014) when biodiversity conservation and ecosystem services are the core area of concern. However, while landform diversity may be a key factor for biodiversity conservation in urban landscapes (q.v. Chapters 2 and 3), it has received little attention in urban biodiversity research and practice. Little is known about landform 
diversity as an indicator to aid landscape architecture researchers and practitioners to make informed decisions regarding the allocation of land to human activities while also attempting to meet biodiversity conservation goals under a climate that continues to change. Modification of natural topography for anthropogenic development, has been, and still is, a challenge in terms of sustainable urban landscape planning and management; this is because less heed has been given to the relationship between landform diversity and land cover and its implications for fauna in urban landscapes, while evidence indicates that topographically diverse patches of vegetation play an important role in species' richness and abundance in Southern Hemisphere urban landscapes (Villegas and Garitano-Zavala, 2010). In Hamilton, New Zealand, research shows that the presence of long-tailed bats (q.v. Table 1.5) is positively correlated with landform diversity (Dekrout et al., 2014). Although evidence from the current literature is widely related to regional scale studies, the interactions in urban contexts between landform diversity and climatic variables remain constant, because ecological processes at regional scales are the same as in urban landscapes (McDonnell and Pickett, 1990; Meurk and Hall, 2006; q.v. Section 1.5).

The vast majority of New Zealand flora and fauna have been historically adapted to cool temperatures (McGlone and Walker, 2011; Meurk et al., 2016). Thus, the identification and proper management of south-facing slopes covered by indigenous land cover classes could be key to protecting the overall level of biodiversity from the local impacts of rising temperatures. Alongside other components of landscape patterns affecting the presence, richness, and abundance of wildlife in urban landscapes (q.v. Table 2.1), and acknowledging the importance of planning for urban green infrastructures to conserve biodiversity and support urban ecosystems (Pauleit et al., 2011; Andersson et al., 2014), it can be argued that topographically diverse patches of vegetation can provide diverse habitat conditions for urban fauna and this, accordingly, will provide opportunities for them to remain in urban landscapes and migrate to more suitable aspects of slopes in the face of warmer than average temperatures without the need to leave urban landscapes completely.

\subsection{MATERIALS AND METHODS}

\subsubsection{Study area}

Wellington has been established and developed in a very diverse landscape of valleys and hills. This provides an opportunity to study landform diversity in relation to land cover. Currently, less than $1 \%$ of the study area comprises expanses of the class Indigenous Forest (q.v. Section 4.5) that is thought to be the primary habitat and food source for fauna in New Zealand (q.v. Chapters 1 
and 3; Appendix V). An increase in temperatures is very likely to affect a wide range of indigenous fauna in New Zealand, including in the study area (Wilson et al., 1998; Chambers, 2004; Pryde et al., 2005; Pierce et al., 2006; McGlone and Walker, 2011; Romijn et al., 2012; Christie, 2014; q.v. Table 1.5).
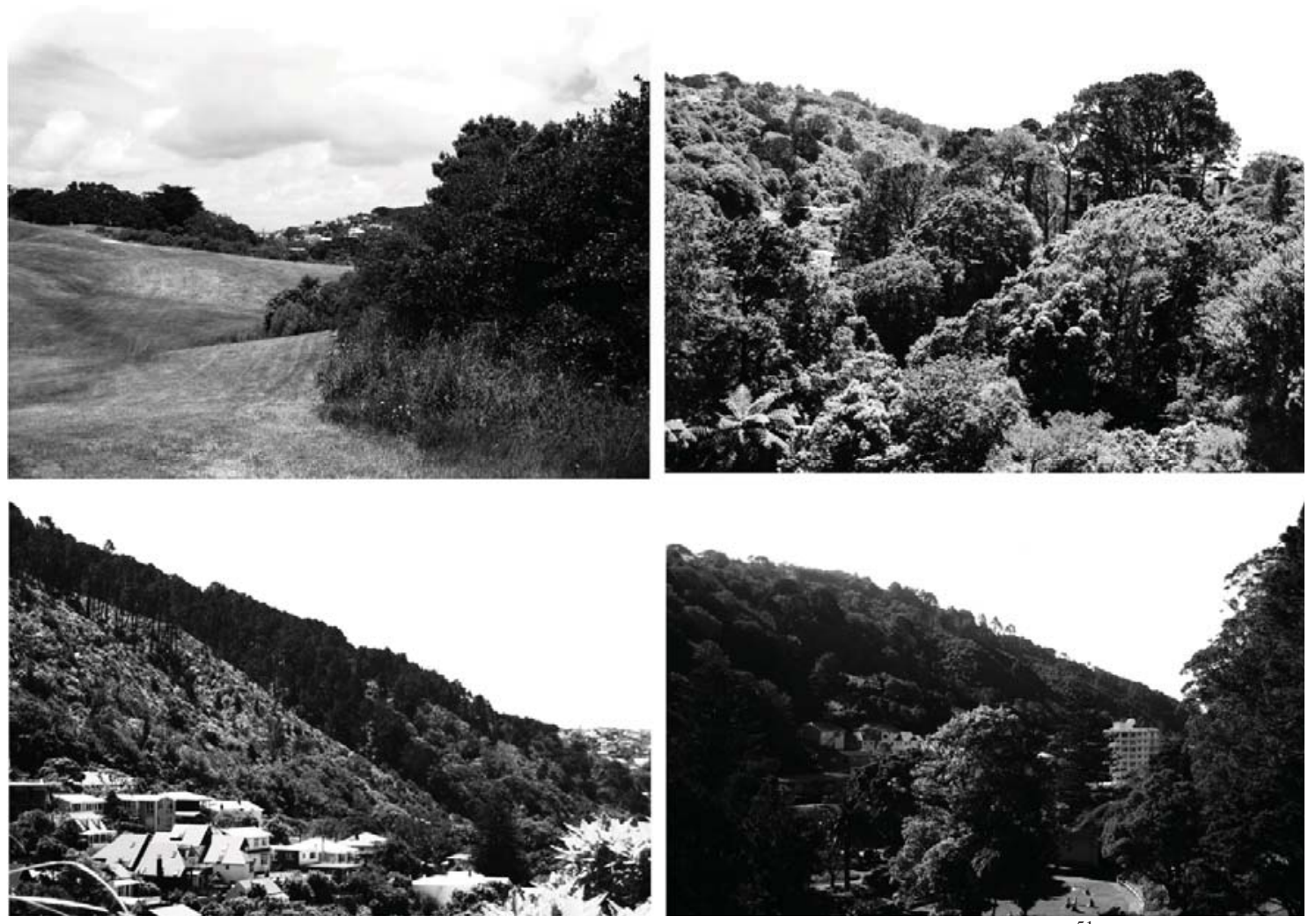

Figure 5.1. Examples of landform diversity in relation to land cover in the Wellington urban landscape ${ }^{51}$.

\subsubsection{Landform and land cover analysis}

Historical trends in urban population change and the rate of urban growth over the last century were studied to provide an accurate picture of the trajectory of change in four key indices (1) total urban land area; (2) urban population; (3) population density; (4) urban land per capita. Once this was complete, Land Cover Data Base v.4.1. - LCDB (Landcare Research, 2015) and the New Zealand North Island Digital Elevation Model 25 m - NZDEM (Landcare Research, 2010) were used as the two main datasets in the process of spatial analysis of the study area (q.v. Section 4.3). The spatial reference used for NZDEM is NZGD 2000 Transverse Mercator (Landcare Research, 2010). The Wellington urban landscape’s aspect map and the Wellington urban landscape’s land cover map

\footnotetext{
${ }^{51}$ Images provided in Figure 5.1 do not refer to certain locations in the study area, but provide a general picture of landform diversity of the Wellington urban landscape.
} 
were created utilising NZDEM and LCDB, respectively. Next, the eight aspects of slope (north, south, west, east, northeast, northwest, southeast, and southwest) were identified using NZDEM. Spatial Analysis Tools including Surface and Reclass were used to identify aspects of slopes. Each aspect was measured in degrees from north. A specific layer was dedicated to each aspect of slope. Each aspect was colour coded using the standard of colours in GIS. In addition, the sixteen existing land cover classes were grouped into four main categories (i.e. Indigenous, Non-indigenous, Extremely-human-modified, and Other) using LCDB (Table 5.1; cf. Table 4.1). Amongst the existing land cover classes, the classes Indigenous Forest and Broadleaved Indigenous Hardwoods are thought to be the most significant habitats that are capable of supporting indigenous flora and fauna (Gabites, 1993; Dawson and Lucus, 2000; Meurk and Hall, 2006; Clarkson et al., 2007; q.v. Chapter 4). Under suitable conditions, the latter class can be transitional towards the former one over time through natural regeneration mechanisms (Landcare Research, 2015; q.v. Chapter 6). These classes are representative of indigenousness in the study area. These two land cover classes were overlaid separately by the Wellington urban landscape’s aspect map in the Arc Map v.10.4.1 environment. Conversion tools were used in the GIS environment in order to enable created maps to be accurately overlaid and adjusted. Overlay analysis was performed using the tool Intersect.

Next, the extent of landform diversity in the study area was mapped and quantified in terms of the eight main directional aspects of slopes to measure the degree to which the entire landscape is topographically diverse. Finally, areas covered by south-facing slopes were overlaid with areas that are currently covered by the classes Indigenous Forest and Broadleaved Indigenous Hardwoods. The main aim was to identify the extent and percentage of the two land cover classes currently overlapping with slopes of various aspects. As discussed above, this analysis was undertaken based upon the key assumption that south-facing slopes in New Zealand may be regarded as a determinant factor to find potential safe havens for indigenous fauna, because they are protected against direct sunlight and solar radiation and are therefore protected, to some extent, from warmer than average temperatures triggered by climate change.

\subsection{RESULTS}

\subsubsection{Change in urban population, density, and land cover}

The human population density in the study area is relatively low (ca. 15 persons per ha) compared to other cities with similar populations around the world (United Nations, 2015). Apart from Wellington's Central Business District (CBD), urban growth and housing development in the 
Wellington urban landscape have historically followed low- or medium density patterns (q.v. Figure 1.5). The human population density in the Wellington urban landscape has decreased dramatically from 23.6 persons per ha in 1886 to 15.02 persons per ha in 2016 (Government Printer, 1887; Statistics New Zealand, 2002; Wellington City Council, 2015). Over this period, the total urban population has experienced a 7.8 times increase whereas the total urban area has become approximately twelve times larger. This shows that the rate of urban population growth as well as trends in urban sprawl in the study area have not increased in an equal and balanced manner. This also reveals that urban development in the Wellington urban landscape has followed a dispersal pattern over the last century (Figure 5.2). Trends in urban expansion since the 1900s have engulfed original forests in Wellington (Pedersen Zari, 2012; 2015; Landcare Research, 2015). Amongst the existing sixteen land cover classes (q.v. Table 5.1), Indigenous Forest and Broadleaved Indigenous Hardwoods cover only approximately 121.7 ha (0.9\%) and 3105.5 ha (22.98\%) of the study area, respectively (Figure 5.3; cf. Figure 4.1 and Table 4.1).

Table 5.1

Percentage of the study area covered by four aggregated land cover classes.

\begin{tabular}{|c|c|c|c|}
\hline Categories & Classes & Area (ha) & Total land area (\%) \\
\hline \multirow[t]{4}{*}{ Indigenous } & Broadleaved Indigenous Hardwoods & 3258 & 24.11 \\
\hline & Indigenous Forest & & \\
\hline & Matagouri or Grey Scrub & & \\
\hline & Herbaceous Freshwater Vegetation & & \\
\hline \multirow[t]{6}{*}{ Non-indigenous } & Gorse and/or Broom & 4785.7 & 35.42 \\
\hline & High Producing Exotic Grassland & & \\
\hline & Exotic Forest & & \\
\hline & Urban Parkland/Open Space & & \\
\hline & Low Producing Grassland & & \\
\hline & Forest Harvested & & \\
\hline \multirow[t]{3}{*}{ Extremely human-modified } & Built-Up Area & 5396.9 & 39.95 \\
\hline & Transport Infrastructure & & \\
\hline & Surface Mine or Dump & & \\
\hline \multirow[t]{3}{*}{ Other } & Sand or Gravel & 70.7 & 0.52 \\
\hline & Lake or Pond & & \\
\hline & Gravel or Rock & & \\
\hline Total & & 13511.3 & 100 \\
\hline
\end{tabular}



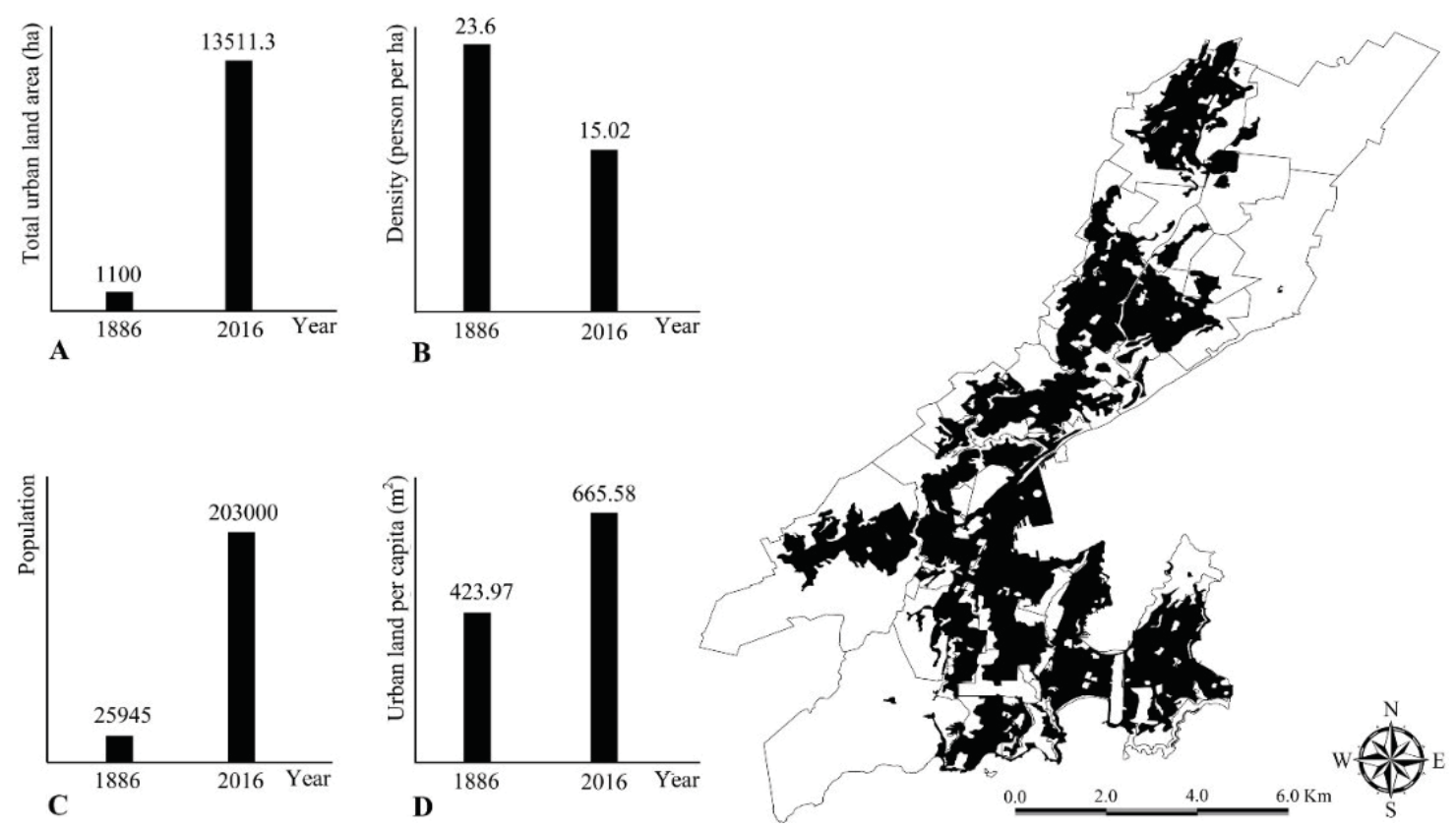

C
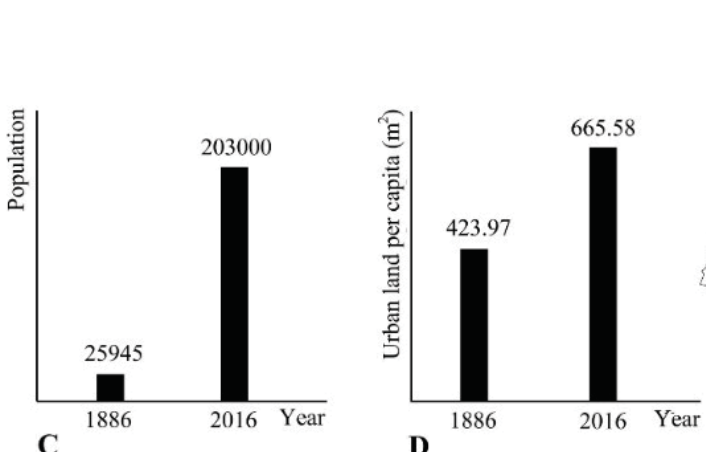

D

Figure 5.2. Population growth in the Wellington urban landscape over time - initial data from Statistics New Zealand (2002) and Government Printer (1887). Map: Dispersal pattern of the class Built-Up Area in Wellington urban landscape in 2015 created based upon Landcare Research (2015).

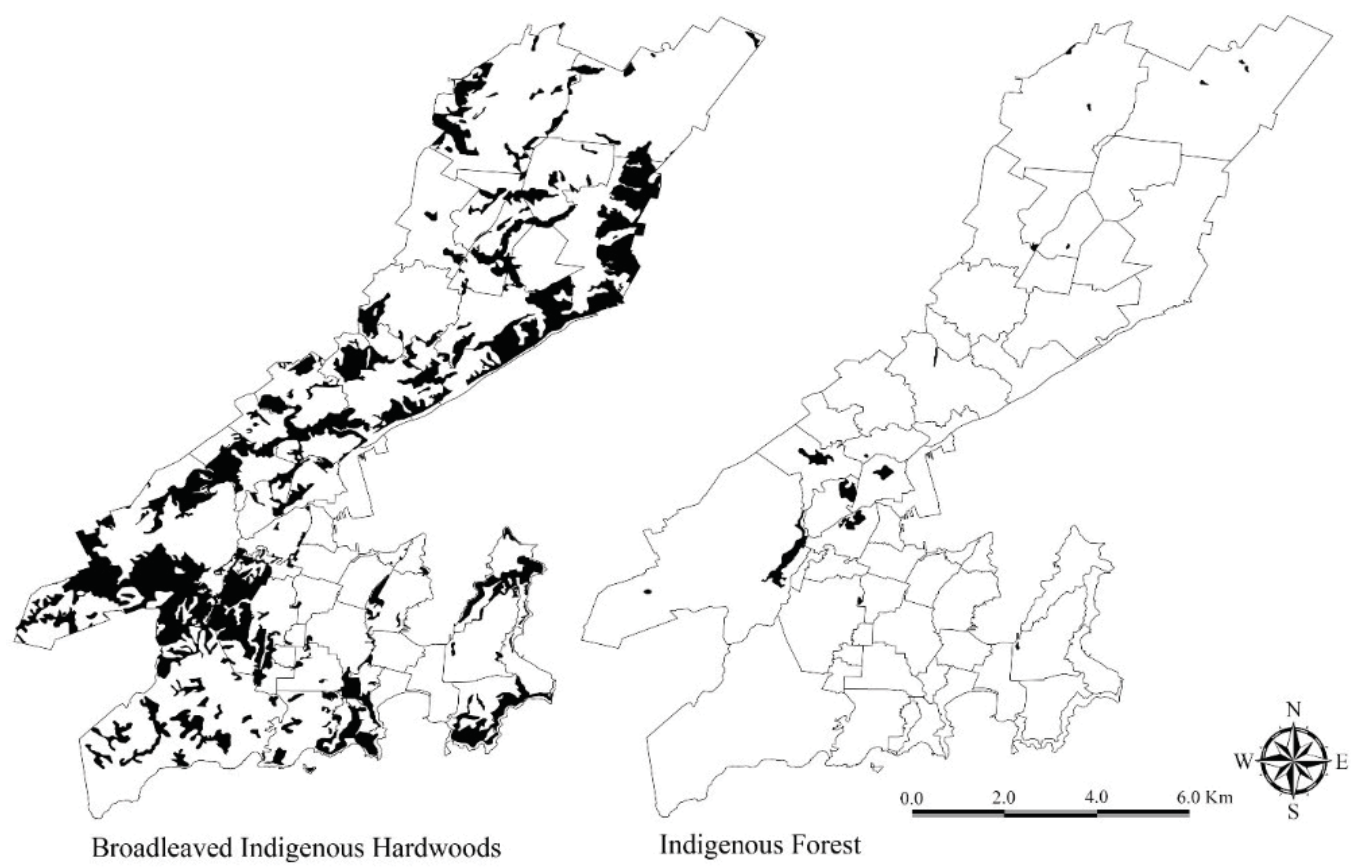

Figure 5.3. The spatial distribution of patches covered by the classes Indigenous Forest and Broadleaved Indigenous Hardwoods (cf. Figure 4.1 and Table 4.1). 


\subsubsection{South facing slopes as urban climate refugia}

The Wellington urban landscape is heterogeneous in terms of landform diversity (Figure 5.4). The most and least predominant aspects of slopes within the boundaries of the study area are eastfacing slopes with 2407.71 ha (17.82\%) and southwest-facing slopes with 1222.77 ha (9.05\%), respectively (Table 5.2). 1226.83 ha (9.08\%), 1424.1 ha (10.54\%), and 1763.22 ha (13.05\%) of the study area are covered by north-, northeast-, and northwest-facing slopes respectively, and cumulatively these cover about one-third of the Wellington urban landscape (32.71\%). South-facing slopes, the theoretically coolest aspect of landscapes in the Southern Hemisphere, cover only 1276.82 ha (9.45\%) of the entire landscape under study. Of the areas of south-facing slopes in the study area, just 416.14 ha (3.04\% of total study area) and 9.13 ha (0.07\% of total study area) are currently covered by Broadleaved Indigenous Hardwoods and Indigenous Forests, respectively (Figure 5.5 and Table 5.3).

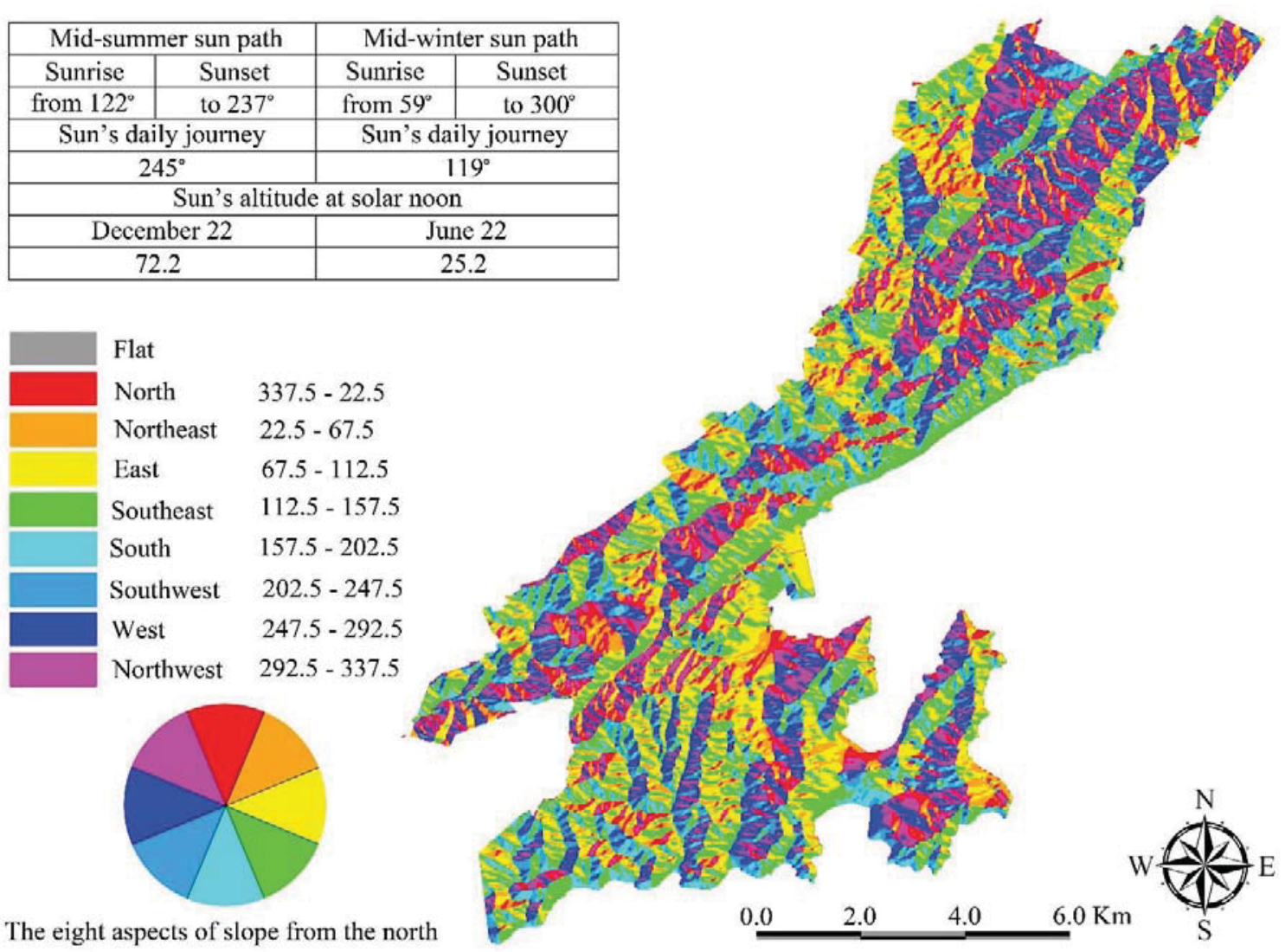

Figure 5.4. Wellington urban landscape aspects of slope - created based upon NZDEM (Landcare Research, 2010). 
Table 5.2

Landform diversity calculated based upon spatial analysis undertaken in the present research illustrating the percentage of each aspect of slope overlapped by the study area, Broadleaved Indigenous Hardwoods (BIH) and Indigenous Forest (IF).

\begin{tabular}{lrrrr}
\hline \multirow{2}{*}{ Aspect of slope } & \multicolumn{4}{c}{ Percentage of the overlapped area by the study area, BIH, and IF } \\
\cline { 2 - 5 } & Study area (ha) & Study area (\%) & BIH (\%) & IF (\%) \\
\hline North & 1226.83 & 9.08 & 6.5 & 8.2 \\
Northeast & 1424.1 & 10.54 & 9.4 & 8.4 \\
East & 2407.71 & 17.82 & 19.5 & 20.5 \\
Southeast & 2207.74 & 16.34 & 22.5 & 25.8 \\
South & 1276.82 & 9.45 & 13.4 & 7.5 \\
Southwest & 1222.77 & 9.05 & 9.8 & 5.8 \\
West & 1982.11 & 14.67 & 10.6 & 7.7 \\
Northwest & 1763.22 & 13.05 & 8.3 & 16.1 \\
Total area & 13511.3 & 100 & 100 & 100 \\
\hline
\end{tabular}

Unlike the class Broadleaved Indigenous Hardwoods, the spatial distribution of the class Indigenous Forest is mostly confined to areas that are already protected by law. Using these areas for future development is therefore unlikely; however, the risk of land cover change as a result of rising temperatures and associated consequences such as wildfire and drought is still high. Weed and pest dispersal remain a critical challenge (q.v. Chapter 3). Table 5.3 shows that 851.55 ha (76.12\%) of the total south-facing slopes are overlapped by other land cover classes that are less important and/or even detrimental to long-term indigenous biodiversity and ecological integrity (q.v. Table 5.3).

In the Wellington urban landscape, the situation is the same for sites of ecological significance including Karori, Khandallah, Red Rock, and Tawa (Our Natural Capital, 2015), where biodiversity can be rich due to environmental variables as well as land cover heterogeneity and higher rates of indigenousness. In these sites, landform is extremely diverse providing micro-ecosystems for urban fauna. In some cases, however, a great proportion of suitable slopes (e.g. south-facing slopes) has been covered by non-indigenous or human-modified land cover classes. Analysis shows that even on a fine scale, the direction of slopes changes continuously in these sites, creating a highly diverse and complex landform, which is essential for ensuring biodiversity. Despite this, a great percentage of land has already been covered by built-up areas and/or patches of vegetation that are of least importance to indigenous fauna. In terms of the rate of indigenousness in relation to landform diversity, the western part of the Wellington urban landscape was identified as the most strategic area for the conservation of biodiversity through spatial planning (Figure 5.6; cf. Figures 6.5, 6.6, 7.7, 7.9 and 8.1; q.v. Chapters 6, 7, and 8). 


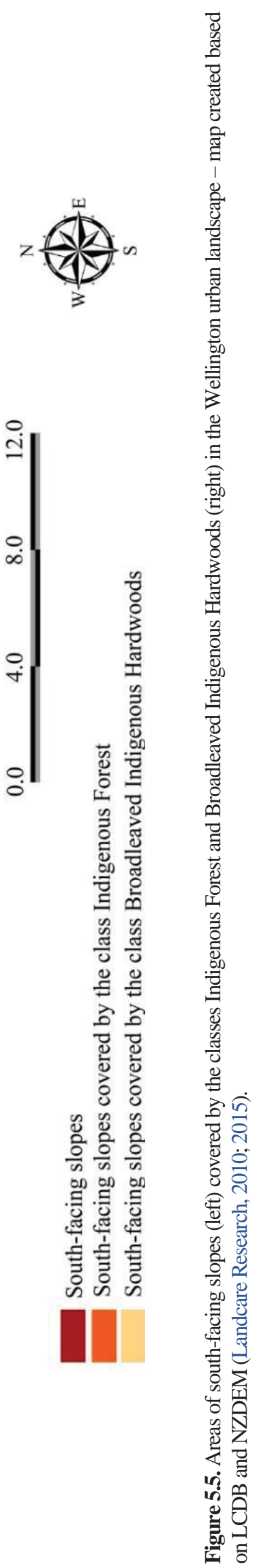




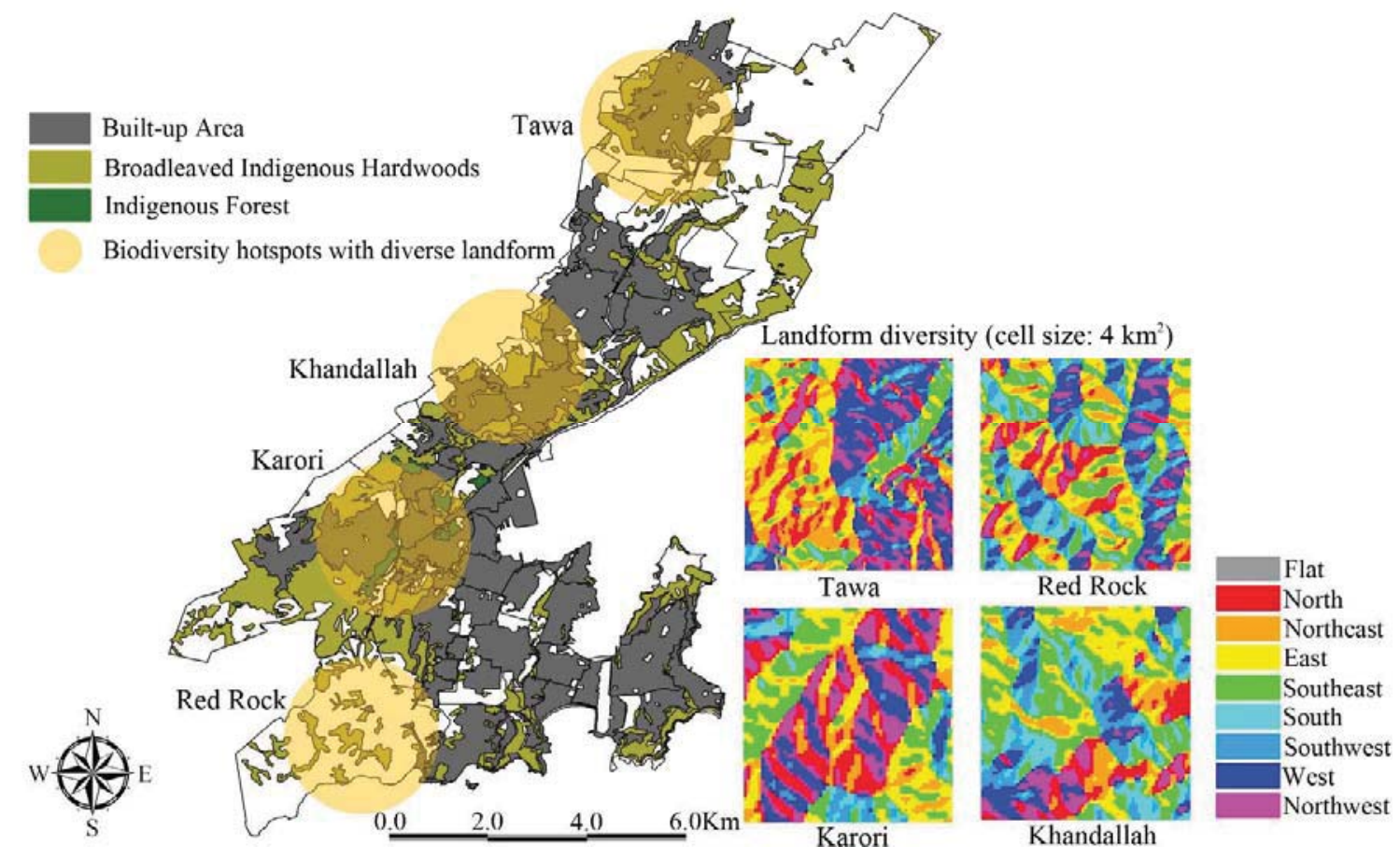

Figure 5.6. Landform diversity in relation to land cover in the Wellington urban landscape's four most important biodiversity hotspots (the cells represent parts of landform diversity in the biodiversity hotspots, not the total areas highlighted by yellow circles).

Table 5.3

Percentage of the south-facing slopes overlaid by the two targeted classes compared to the study area.

\begin{tabular}{|c|c|c|c|}
\hline Class & Area (ha) & Total land area (\%) & Overlapping with south-facing slopes \\
\hline Broadleaved Indigenous Hardwoods & 3105.5 & 22.98 & $\begin{array}{r}\approx 416.14 \text { ha } \\
\approx 13.4 \% \text { of the class } \\
\approx 3.07 \% \text { of the study area }\end{array}$ \\
\hline Indigenous Forest & 121.7 & 0.9 & $\begin{array}{r}\approx 9.13 \text { ha } \\
\approx 7.5 \% \text { of the class } \\
\approx 0.06 \% \text { of the study area }\end{array}$ \\
\hline Other land cover classes & 10284.1 & 76.12 & $\begin{array}{l}\approx 851.55 \text { ha } \\
\approx 8.3 \% \text { of the other classes } \\
\approx 6.32 \% \text { of the study area }\end{array}$ \\
\hline Total & 13511.3 & 100 & $\begin{array}{r}1276.82 \text { ha } \\
\approx 9.45 \% \text { of the study area }\end{array}$ \\
\hline
\end{tabular}

\subsection{DISCUSSION}

Spatial analysis of landform in relation to land cover can reveal the degree to which an urban landscape may support indigenous fauna in the face of rising temperatures where aspects of slopes have determinant implications for urban fauna. Over the last century, considerable percentage of 
slopes of conservation significance (i.e. south-facing slopes) in the Wellington urban landscape has been affected by urban development and the dispersal of exotic flora.

The significance of environmental factors such as natural and anthropogenic edge effects in influencing biodiversity in urban landscapes is discussed in detail (q.v. Chapters 7 and 8). Aside from this, vulnerable fauna in the Wellington urban landscape are potentially threatened by two important challenges in the face of rising temperatures when landform diversity and land cover are concurrently scrutinised. First, of sixteen land cover classes currently present in the study area, just four are classified as indigenous, covering 3258 ha (24.11\%) of the total area (cf. Table 4.1). Loss of biodiversity at an ecosystem level (i.e. a decrease in the extent and number of indigenous land cover classes), and consequently loss of suitable habitats for a wide range of indigenous flora and fauna, is therefore very likely. Second, only 1276.82 ha (9.45\%) of the total urban landscape is covered by south-facing slopes that can serve as relatively protected habitats in the face of rising temperatures. Of this, 851.55 ha (76.12\%) of the south-facing slopes are covered by fourteen land cover classes that cannot contribute significantly to biodiversity conservation (q.v. Table 5.3). In practice, the process of decision-making on the allocation of land to human activities and/or biodiversity conservation involves complex trade-offs between different objectives and values.

For Wellington, approximately 21,400 additional dwellings are needed in response to the projected population growth in the study area by the year 2043 (Wellington City Council, 2015). If managed improperly, the allocation of land for this relatively large amount of housing development could give rise to greater urban sprawl and this, in itself, will affect potentially suitable places that urban fauna might use as safe havens as temperatures rise (i.e. south-facing slopes overlapping the classes Indigenous Forest and Broadleaved Indigenous Hardwoods). If managed appropriately, the existing landform diversity in the study area may contribute in two important ways to biodiversity conservation, even under rising temperatures triggered by climate change. First, due to the diversity of aspects of slopes in the study area, different environmental conditions, or micro-climates, are likely to be created and should be recognised and protected for the strategic benefit of urban fauna in light of the uncertainties and dynamics associated with climate change.

As shown, the spatial distribution of slope aspects is heterogeneous, widely scattered across the Wellington urban landscape, even at the fine scale. This geo-spatial characteristic can potentially facilitate the migration of fauna from warmer patches of vegetation to cooler ones as temperatures rise, or as more frequent extreme climate change driven events occur. This could ensure that a greater range of urban habitats exists with correspondingly different microclimates that will be useful habitats 
in different climatic circumstances. Second, given that much indigenous fauna in New Zealand has been historically adapted to cool temperatures (McGlone and Walker, 2011; Meurk et al., 2016), the proactive planning and management of south-facing slopes, especially those that are covered by indigenous land cover classes, may be regarded as an opportunity to help in safeguarding, or at least supporting, the overall level of urban biodiversity against the ill-effects of rising temperatures. This goal can be achievable if the commitment to weed and pest control is strategically fulfilled and landscape restoration practices are concurrently implemented in targeted locations (Norton et al., 2016; q.v. Chapter 6). This spatial strategy may help to ensure the presence of fauna in urban landscapes even when rising temperatures may create unsuitable conditions for them elsewhere.

In a New Zealand context, which has relatively cool temperatures year-round, availability of direct sunlight is considered essential for people. Direct sunlight occurs predominantly on northfacing slopes, which makes those slopes ideal for housing development while south-facing slopes have been shown as being ideal for protecting flora and fauna in an era of climate change. Thus, prioritising south-facing slopes for biodiversity conservation and north-facing ones (that are not currently covered in indigenous land cover classes) for humans could be a 'win-win' strategy in landscape architecture and land use planning practices.

Wellington is New Zealand's pioneer city in terms of urban ecosystem restoration (Clarkson and Kirby, 2016). Utilising maps created in the process of this research may also be beneficial in the process of landscape restoration practices (Williams et al., 2011; Meurk et al., 2016). In-fill urban development may need to be carefully considered in the coming decades to enable strategic decisions to be made about where new urban development should occur, while concurrently preserving southfacing slopes of ecological significance alongside built-up areas. In the Wellington urban landscape, previously affected by land cover change and dispersed urban development, the allocation of land to a wide range of human land use activities should be undertaken with particular attention to the scarcity of expanses of indigenous land cover classes overlapping with south-facing slopes.

\subsection{CONCLUSION}

The coupled stress of urbanisation and global warming has profound impacts on biodiversity. As shown, the spatial analysis of landform in relation to land cover is a key vehicle in measuring the capability of urban landscapes for providing suitable habitats for those vulnerable fauna suffering from rising temperatures, particularly in cities where topography plays a key role in the spatial distribution of land cover classes and human land use activities. Although protecting south-facing 
slopes does not guarantee that biodiversity will be able to adapt, this study reveals that there are still potential opportunities for establishing urban strategies that can help ensure the conservation of wildlife against the impacts of rising temperatures. Landform diversity and indigenous land cover cumulatively may be able to fulfil an important role in biodiversity conservation particularly in the western part of the Wellington urban landscape.

Information gained in the course of this research will be able to be utilised in the process of landscape architecture, land use planning, and sustainable urban design practices. The methodology undertaken in this research can be applied by landscape architecture researchers to provide a robust scientific basis for making informed decisions regarding the allocation of land to human land use activities in urban landscapes in a way that supports, or at least does minimal harm to, indigenous biodiversity. This will also work to avoid further habitat loss and accordingly provide urban fauna with a greater chance of being able to remain in cities and respond to rising temperatures more effectively, thus helping to minimise the need for wildlife emigration from urban landscapes. As discussed in Chapter 4, landscape restoration practices may contribute to natural regeneration. For example, the class Gorse and/or Broom can be replaced by the classes Broadleaved Indigenous Hardwood and then Indigenous Forest over time (q.v. Chapter 4). Therefore, there is a need for quantifying and measuring aspects of slope covered by the class Gorse and/or Broom in order to identify the potential for natural regeneration in relation to landform diversity. Research is required to find potential areas for natural regeneration in the study area that are best placed to contribute to indigenous biodiversity in the future. As connectivity and proximity is vital to support landscape restoration through natural regeneration mechanisms, this component of landscape pattern should also be taken into particular consideration. These issues will be addressed in detail in Chapter 6 . 


\section{CHAPTER 6 \\ Study III: Landscape restoration through natural regeneration mechanisms}

\subsection{RESEARCH AIMS}

In Chapter 4, results revealed that just $0.9 \%$ (121.7 ha) of the Wellington urban landscape is currently covered by the land cover class Indigenous Forest. This class has been shown to provide indigenous fauna with primary habitats and food sources (q.v. Chapter 3). In Chapter 5, it was shown that just 9.13 ha of this class, equivalent to $0.06 \%$ of the study area, overlap with south-facing slopes that are thought to be vitally important for safeguarding, or at least supporting, a wide range of indigenous fauna against the negative effects of rising temperatures (q.v. Chapter 5). Overall, discussions held with New Zealand subject-matter experts (q.v. Chapter 3), and results derived from spatial analysis of land cover in Chapters 4 and 5, suggest landscape restoration through natural regeneration mechanisms is an alternative response to indigenous biodiversity loss in the study area. Thus, the main aims of this chapter are to:

(1) Provide spatial information about the status and extent of land cover classes in the study area - the Wellington urban landscape (q.v. Figure 1.6), before the arrival of humans in order to provide a baseline for ecosystem and land cover change analysis;

(2) Address spatial opportunities for landscape restoration through natural regeneration mechanisms with particular attention to connectivity and proximity between patches of vegetation as well as the key role of the class Gorse and/or Broom;

(3) Suggest a four-step process for spatial analysis of land cover classes in order to identify the potential for natural regeneration in support of indigenous biodiversity in an era of climate change. 


\subsection{INTRODUCTION}

Landscape restoration has been recommended for the Southern Hemisphere to avoid further land cover change, habitat loss, and accordingly, biodiversity loss (Walker et al., 2007; Chazdon and Uriarte, 2016; Wardell-Johnson et al., 2016; Schulz and Schröder, 2017). Concurrently, landscape restoration is likely to be an effective response to climate change impacts in the coming decades (Harris et al., 2006). From a well-being perspective, Standish et al. (2013) argue that irrespective of the current gap between ecological knowledge and land use planning practices in urban landscapes, landscape restoration in cities would necessarily be an integral part of urban planning and management in the future to increase society's interaction with nature. Furthermore, landscape restoration in cities can contribute to a higher level of urban ecosystem services and consequently human well-being (Beatley, 2016; Wu, 2014).

In New Zealand, landscape restoration through natural regeneration in human-modified landscapes is necessary to support the country's indigenous biodiversity (Meurk and Swaffield, 2000). For at least two decades, landscape restoration on an urban scale has been an area of research and practice in New Zealand (Clarkson and Kirby, 2016). Strategies include pest control, revegetation, and land use planning for natural regeneration. A survey of 228 landscape restoration projects in New Zealand shows that 18.1\% have been conducted in urban landscapes (Peters et al., 2015). Landscape restoration through natural regeneration is likely to be a cost-effective strategy to tackle biodiversity decline where there is not sufficient funding to invest in other strategies. According to Norton et al. (2016), public support and political will are also two important factors underpinning landscape restoration practices. These socio-political factors seem to be potentially available in New Zealand (Lyver et al., 2015; Department of Conservation, 2016; Sullivan and Molles, 2016).

\subsubsection{Seed dispersal mechanisms}

Seed dispersal by avifauna is an integral part of natural regeneration in Southern Hemisphere ecosystems (Pizo, 1997; Catterall et al., 2004; Garcia et al., 2010; Mariano and Christianini, 2016). In New Zealand, seeds of up to $70 \%$ of the ca. 240 forest species of woody plants can be dispersed by avifauna (Clout and Hay, 1989). Thus, the absence of birds is very likely to affect this mechanism.

Since ca. 1000 CE, New Zealand has lost a substantial percentage of its indigenous fauna including $40-50 \%$ of the avifauna as a result of human interventions in landscapes (Holdaway, 1989). Some extinct species, such as moa (Dinornithidae 12 spp.), huia (Heteralochaacutirostris), and piopio (Turnagracapensis), were among the principal seed dispersers for a wide range of indigenous flora, 
specifically large-fruited plant species, due to their relatively large gapes of $>5 \mathrm{~cm}, 1.5 \mathrm{~cm}, 1.1 \mathrm{~cm}$, respectively (Clout and Hay, 1989). Due to the relationship between fruit diameter and species gape size, seed dispersal of New Zealand's ecologically important indigenous trees with fruits $>14 \mathrm{~mm}$ diameter currently depends, to a large extent, on the still relatively abundant kererū (Hemiphaganovaeseelandiae) (Clout and Hay, 1989) and to some extent on tūī (Prosthemadera novaeseelandiae) (Kelly et al., 2010). Although research shows the presence of lizards, as pollinators and/or seed dispersers, is also important to the ecosystems in which they live (Webb and Kelly, 1993; Whitaker, 1987; Wotton, 2002; Wotton et al., 2016; Chapple, 2016), little is yet known about their role in pollination and seed dispersal in urban landscapes.

\subsubsection{Functional connectivity}

There is sufficient evidence to show that connectivity and proximity is vital to support avifauna movement, plant colonisation, gene flow, and seed dispersal mechanisms (Shaffer, 1981; Forman, 1995; Bennett, 1999; Honnay et al. 2003; q.v. Tables 2.1 and 2.2). The distance from seed source (i.e. areas covered by indigenous plants) to deposit site (i.e. a potential site of natural regeneration), as well as the ability of seed dispersers to carry seeds between these locations, seems to be a key factor determining the potential of landscape patterns to facilitate or hinder the process of natural regeneration. At the same time, connectivity and proximity could facilitate the dispersal of weeds and pests (Barnes, 2000; Botequilha Leitao et al., 2006). In line with seed dispersal and pollination for the benefit of indigenous biodiversity resulting from functional connectivity between patches of vegetation and proximity between seed source and deposit site, there is also concern that dispersal of woody weeds occurs in the same way in urban New Zealand (Sullivan et al., 2009).

Although kererū and tūi actively serve as seed dispersers of indigenous flora, they are concurrently amongst those species that disperse weed seeds (Wotton and McAlpine, 2015). This paradoxical function, could be alleviated by increasing the percentage of indigenous land cover and thereby reducing sources of exotic seeds. Although pest and weed control should continue in support of indigenous biodiversity, informed landscape architecture practices are required for biodiversity conservation in urban New Zealand, even though the current knowledge concerning spatial requirements of wildlife species as well as essential spatial connectivity and proximity for natural regeneration is not yet sufficient (Meurk et al., 2016; q.v. Section 3.3.1.6). Kererū can fly distances of between 11.4 and 101.9 kilometres and therefore reach relatively distant locations (Powlesland et al., 2011). Thus, this species can act as an important seed disperser in areas where patches of indigenous vegetation have been fragmented. Kererū can disperse between 66 and 87\% of seeds away from the 
seed source depending on the fruits of the plant species being eaten. In addition, the seed dispersal distances for several indigenous trees were recorded as being between 61 and $95 \mathrm{~m}$. Less than 1\% of seeds, however, were estimated to be dispersed $>1 \mathrm{~km}$ from the seed source (Wotton and Kelly, 2012). This result agrees with that of a study of nine indigenous wind- and bird-dispersed plant species suggesting that when distance from seed source is $<100 \mathrm{~m}$, the chance of natural colonisation of indigenous plant species is likely to be higher (Sullivan et al., 2009). Therefore, while kererū and tūì can fly long distances, normally they cannot disperse seeds more than ca. $100 \mathrm{~m}$ from the seed source.

\subsubsection{The ecological importance of the class Gorse and/or Broom}

The original range of gorse (Ulex europaeus) and broom (Cytisus scoparius) is from the United Kingdom to the western part of Spain, France, and Portugal (Syrett et al., 1999). These species were imported to many Southern Hemisphere countries between 1775 and 1940 (Atlan et al., 2015). In New Zealand, gorse and broom were first recorded in 1867 and 1872, respectively (Webb et al., 1988). Gorse was sometimes used to define property boundaries in urban New Zealand (Myers and Bazely, 2003) but became a highly problematic weed. Not surprisingly, today a high percentage of New Zealand's urban landscapes is covered by this class (Figure 6.1; q.v. Table 4.1). Gorse and broom can, however, be utilised as a nurse crop (Davis and Meurk, 2001) to facilitate the process of ecological succession towards indigenous forests because they provide the shade and windbreak conditions needed for native seedlings to flourish. Thus, areas covered by this class can potentially be regarded as deposit sites.

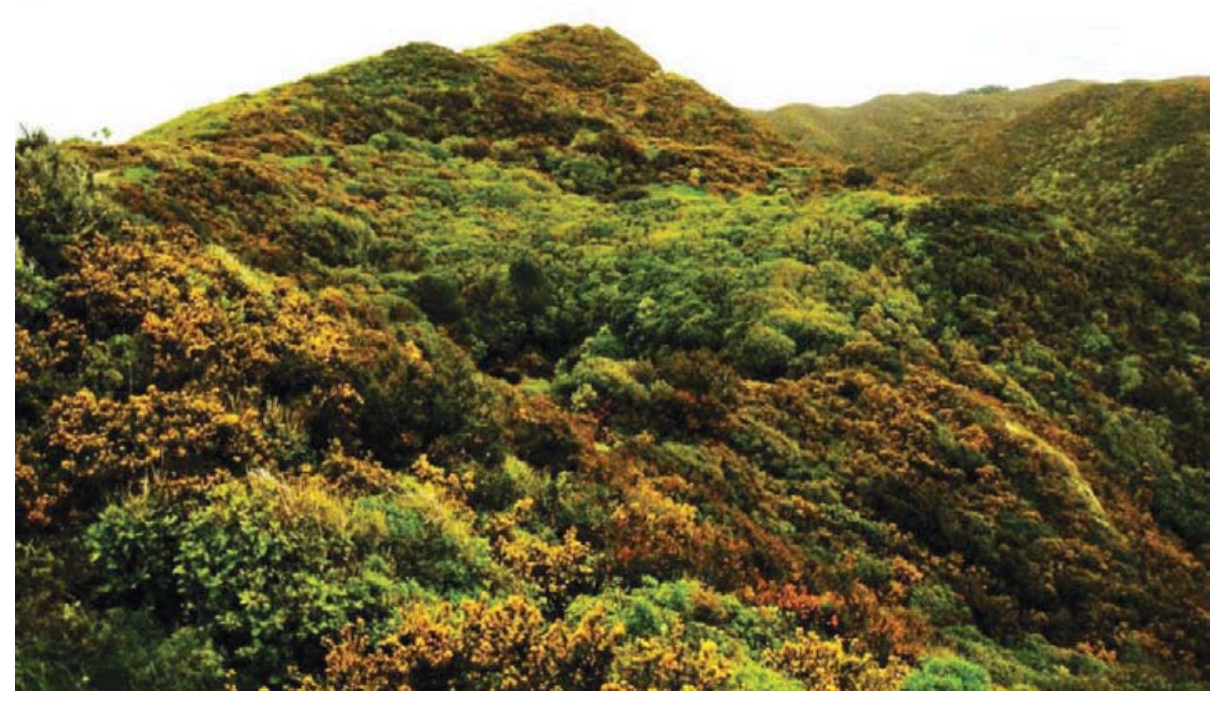

Figure 6.1. Areas covered by the class Gorse and/or Broom at the edge of the Wellington urban landscape 
In New Zealand, the three bio-environmental factors: (1) rainfall, (2) topography in relationship to temperature and sunlight radiation and direction, and (3) distance from seed source, play a major role in speeding up natural regeneration processes (Davis and Meurk, 2001; Williams, 2011). In the Southern Hemisphere, south- and then, west- and east-facing aspects of slopes provide moist and cool conditions that accelerate the process of ecological succession and consequently natural regeneration in areas covered by the class Gorse and/or Broom (Davis and Meurk, 2001). For example, Williams (2011) suggests that under suitable conditions as described by McCracken (1993), gorse can be replaced by some indigenous broadleaved species in 20 to 30 years, while moist and cool aspects, such as south-facing slopes, are potentially capable of natural regeneration in 10 to 20 years (Davis and Meurk, 2001; cf. Wotton and McAlpine, 2013). These bio-environmental factors play a key role in the natural regeneration of Southern Hemisphere urban landscapes (Figure 6.2).

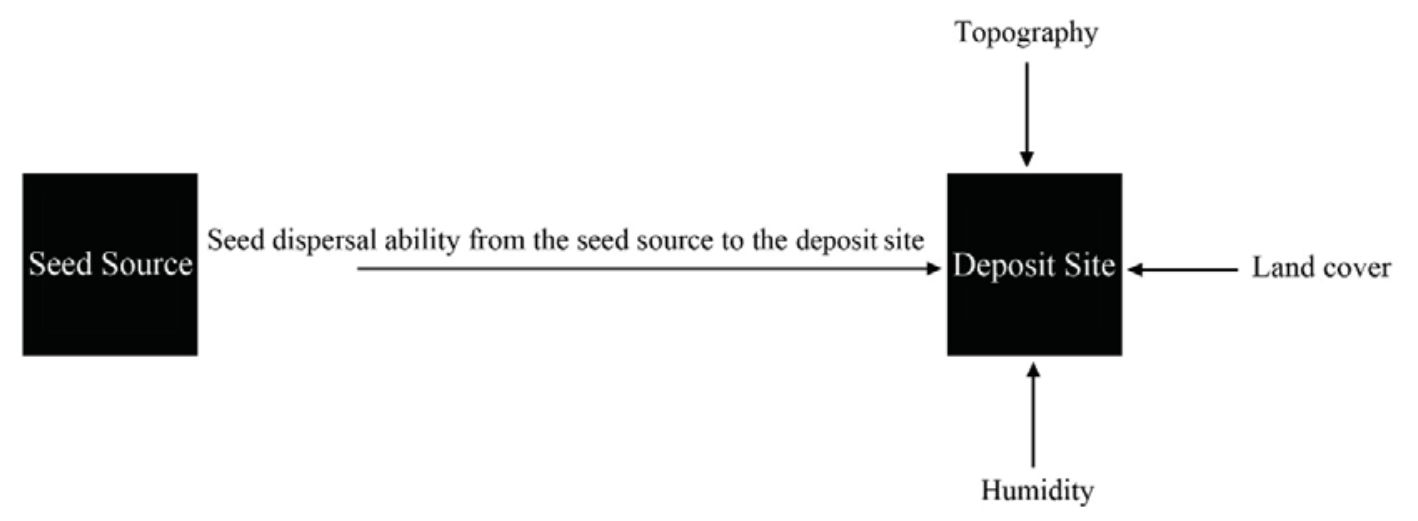

Figure 6.2. Key bio-environmental factors in the process of landscape restoration through natural regeneration

\subsection{MATERIALS AND METHODS}

Arc Map v.10.4.1 was used to provide a spatially-explicit depiction of the extent of different types of ecosystems in the Wellington urban landscape in the pre-human period (i.e. pre-human land cover classes) to compare the historical status with the current situation utilising two sets of GIS-based data, LCDB (q.v. Section 4.3) and Potential Vegetation of New Zealand - PVNZ (Landcare Research, 2012; 2015). PVNZ is a spatial database of the distribution of major canopy tree species before human interventions in New Zealand landscapes (Landcare Research, 2012; Appendix VI). PVNZ was used to create a new map of the study area's land cover in the pre-human period. LCDB was used to depict the current spatial distribution of land cover classes.

Using LCDB, the vegetation class Gorse and/or Broom was quantified as a potentially suitable class for natural regeneration (Williams 2011; Landcare Research, 2015). Areas covered by this class potentially act as deposit sites. NZDEM (q.v. Section 5.3.2) was used to identify aspects of 
slopes covered by the class Gorse and/or Broom (Landcare Research, 2010). NZDEM was overlaid with areas covered by the class Gorse and/or Broom to identify the percentage of each aspect of slope covered by the class.

To measure the potential for natural regeneration through the dispersal of seeds, the ability of kererū and tūî to disperse seed from the seed source (i.e. areas covered by the class Indigenous Forest) to the deposit site (i.e. areas covered by the class Gorse and/or Broom) was taken into consideration as an indicator for spatial analysis ( $\geq 100 \mathrm{~m}$; q.v. Section 6.2.2). Maps created based upon LCDB were used to quantify and measure the spatial distances between seed sources and deposit sites.

\subsection{RESULTS}

\subsubsection{Analysis of the pre-human landscape}

Almost the entire study area was covered by five types of indigenous forests before the arrival of humans (Figure 6.3). Of 24 land cover classes identified as pre-human environments of New Zealand (Leathwick et al., 2004; Landcare Research, 2012), five classes namely (1) Rimu/tawakāmahi forest, (2) Kahikatea-mātai/tawa-māhoe forest, (3) Rimu-miro/kāmahi-red beech-hard beech forest, (4) Kahikatea-pukatea-tawa forest, and (5) Rimu-mātai-miro-tōtara/kāmahi forest were present in the study area. More than $85 \%$ of the study area was dominated by the class Rimu/tawa-kāmahi Forest. The other four classes consisted of less than $12 \%$ of the study area. In addition, $2.9 \%$ of the study area was unclassified (Table 6.1). Standard definitions of the land cover classes in the prehuman period have been provided in Leathwick et al. (2004) and Landcare Research (2012). Today, these five forest types (i.e. pre-human land cover classes) are collectively classified under the class Indigenous Forest (Landcare Research, 2015).

\subsubsection{Analysis of the current landscape}

Less than one-fourth of the study area is currently dominated by four indigenous land cover classes. Broadleaved Indigenous Hardwoods, Indigenous Forest, Matagouri or Grey Scrub, and Herbaceous Freshwater Vegetation cover 22.98, 0.9, 0.22, and $<0.02 \%$ of the study area, respectively (q.v. Table 4.1). The rest of the study area (ca. 75\%) has been converted to other land cover classes including built-up areas and five classes of exotic vegetation (Table 6.2; cf. Table 4.1). 
Table 6.1

The extent of pre-human land cover classes in the Wellington urban landscape - calculated based upon PVNZ (Landcare Research, 2012).

\begin{tabular}{lrr}
\hline Land cover classes & z Area (ha) & \% of total landscape \\
\hline Rimu/tawa-kāmahi Forest & 11531 & 85.3 \\
Kahikatea-mātai/tawa-māhoe Forest & 1092 & 8.1 \\
Rimu-miro/kāmahi-red beech-hard beech Forest & 346 & 2.5 \\
Kahikatea-pukatea-tawa Forest & 144 & 1.1 \\
Rimu-mātai-miro-tōtara/kāmahi Forest & 19 & 0.1 \\
Unclassified & 397 & 2.9 \\
Total landscape & 13511 & 100 \\
\hline
\end{tabular}

Table 6.2

Current land cover classes in the Wellington urban landscape - calculated based upon LCDB (Landcare Research, 2015).

\begin{tabular}{lrr}
\hline Land cover classes & z Area (ha) & \% of total landscape \\
\hline Broadleaved Indigenous Hardwoods & 3105.5 & 22.98 \\
Indigenous Forest & 121.7 & 0.9 \\
Matagouri or Grey Scrub & 30.4 & 0.22 \\
Herbaceous Freshwater Vegetation & 0.4 & $<0.02$ \\
Gorse and/or Broom & 1693.3 & 12.53 \\
Other classes including other exotic classes & 8559.7 & $>63.36$ \\
Total landscape & 13511 & 100 \\
\hline
\end{tabular}

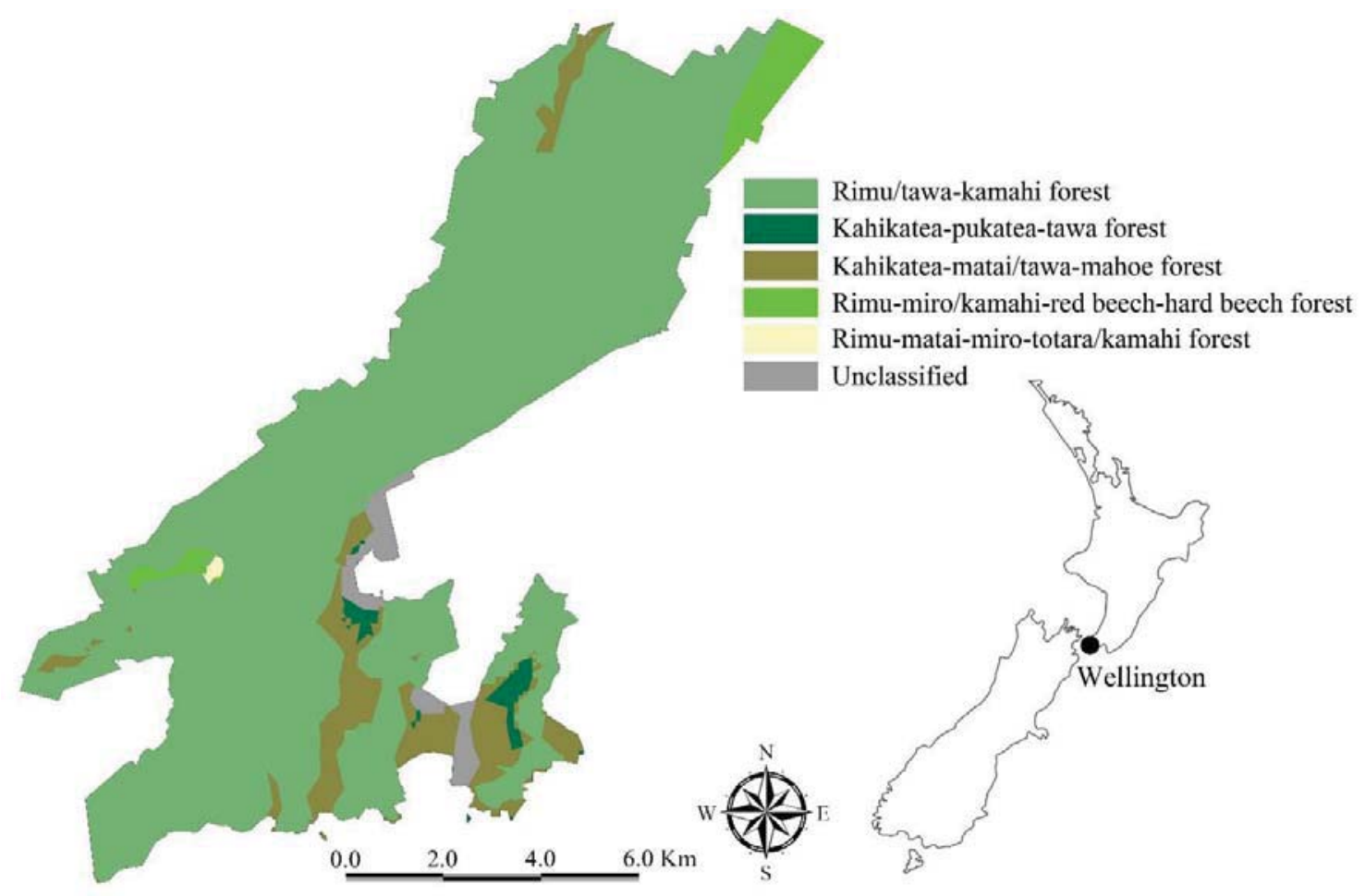

Figure 6.3. Pre-human land cover classes in the Wellington urban landscape - map created based upon PVNZ (Landcare Research, 2012). 


\subsubsection{Topography and the class Gorse and/or Broom}

Of the 1693.3 ha (12.53\%) of the study area covered by the class Gorse and/or Broom, 189.65 ha (11.2\%) overlay south-facing slopes. In addition, 240.11 ha (14.18\%) and 211.66 ha (12.5\%) of the class dominate the southeast- and southwest-facing slopes, respectively. East- and west-facing slopes are the second most important aspects of slopes in terms of capability for landscape restoration through natural regeneration from gorse and broom to some indigenous species. There are collectively 525.6 ha (31.04\%) of the class on east- and west-facing slopes. In addition, north-, northeast-, and northwest-facing slopes, the most unsuitable aspects of slope in the study area for ecological succession from gorse and broom covered land, are collectively covered by a similar area (526.28 ha, 31.08\%) of the class (Table 6.3; Figure 6.4).

Table 6.3

Aspects of slope covered by the class Gorse and/or Broom - calculated based upon LCDB (Landcare Research, 2015) and NZDEM (Landcare Research, 2010).

\begin{tabular}{lrr}
\hline Aspects of slope & Area (ha) & Percentage \\
\hline North & 142.24 & 8.4 \\
Northeast & 166.11 & 9.81 \\
East & 249.93 & 14.76 \\
Southeast & 240.11 & 14.18 \\
South & 189.65 & 11.2 \\
Southwest & 211.66 & 12.5 \\
West & 275.67 & 16.28 \\
Northwest & 217.93 & 12.87 \\
Total area & 1693.3 & 100 \\
\hline
\end{tabular}

\subsubsection{Connectivity and distance between seed sources and deposit sites}

Spatial analysis of connectivity and proximity reveals an important ecological area in the western part of the Wellington urban landscape (i.e. the ecological hub, q.v. Chapter 5) consisting of eight patches covered by the class Indigenous Forest (Figures 6.5 and 6.6). The total area of the Wellington urban landscsape is covered by 121.7 ha of the class Indigenous Forest (q.v. Table 4.1). This class can be considered as the main source of seed for the natural regeneration of indigenous plants and is also primary habitat for important seed dispersers such as kererū and tūî. Of this coverage, the ecological hub includes eight patches of indigenous forest cumulatively covering 106.9 ha of the area by the class Indigenous Forest (Table 6.4). This is equivalent to more than $87.8 \%$ of the class in the entire study area (cf. Table 6.2). 

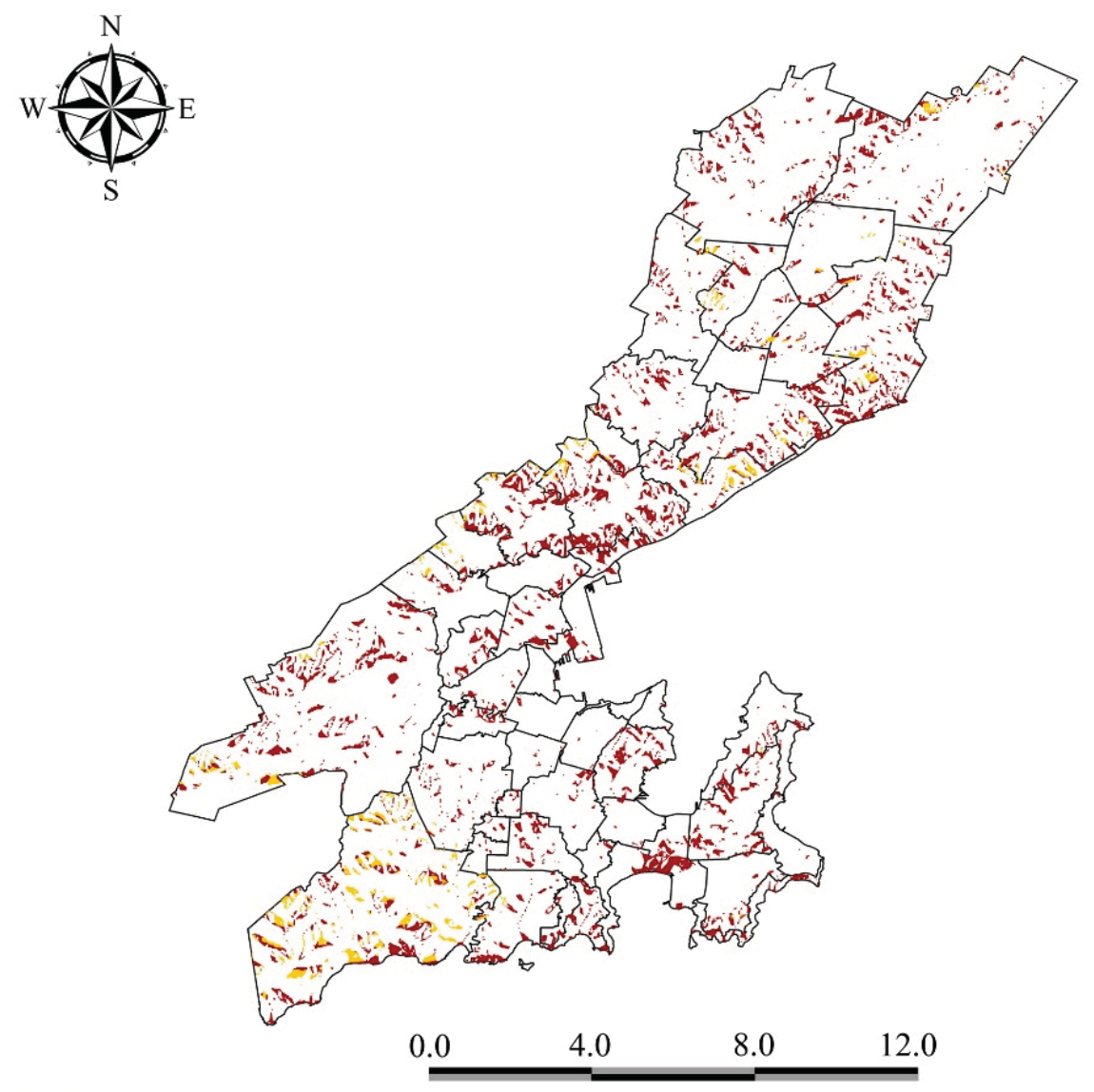

South-facing slopes

South-facing slopes covered by the class Gorse and/or Broom

Figure 6.4. Areas of south-facing slopes covered by the class Gorse and/or Broom in the Wellington urban landscape - map created based upon LCDB and NZDEM (Landcare Research, 2010; 2015).

Table 6.4

The eight patches of indigenous forest identified in the ecological hub (cf. Figure 6.6).

\begin{tabular}{lr}
\hline Patches of indigenous forest & Area $\left(\mathbf{m}^{2}\right)$ \\
\hline A & 17.76 \\
B & 0.81 \\
C & 10.32 \\
D & 17.56 \\
E & 15.71 \\
F & 40.78 \\
G & 2.07 \\
H & 1.89 \\
Total & 106.9 \\
\hline
\end{tabular}




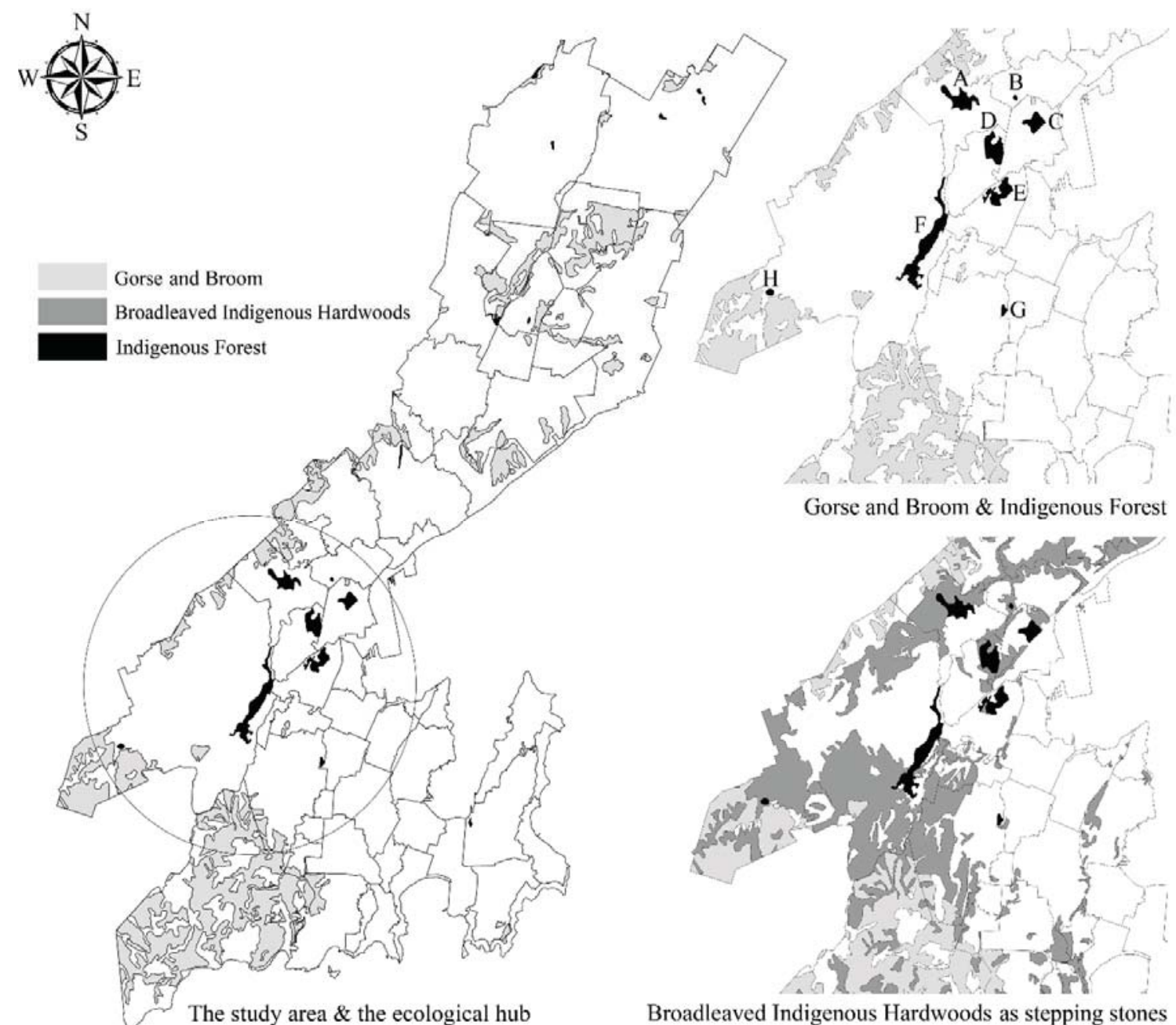

Figure 6.5. Spatial composition and configuration of the ecological hub in the western part of the Wellington urban landscape in relation to the two classes Gorse and/or Broom and Broadleaved Indigenous Hardwood - maps created based upon LCDB (Landcare research, 2015).

A relatively clustered pattern of these patches of indigenous forest in the ecological hub provides opportunities for landscape restoration through natural regeneration. The nearest edge-toedge distances between the eight patches of indigenous forest vary from $278 \mathrm{~m}$ to $2489 \mathrm{~m}$ (q.v. Figure 6.6). Patches covered by the class Broadleaved Indigenous Hardwoods can also theoretically be used by seed dispersers as stepping stones owing to their typical spatial compositions and configurations in relation to the classes Indigenous Forest (i.e. seed source) and Gorse and/or Broom (i.e. deposit site) (q.v. Figure 6.5 - right bottom).

In urban New Zealand, ecologically suitable distances between patches $\geq 6.25$ ha and $\geq 1.56$ ha are 5000 m and between 1000 and 2000 m, respectively (Meurk and Hall, 2006; q.v. Chapter 3). This spatial requirement is met in the ecological hub, theoretically providing suitable connectivity and proximity between seed sources (q.v. Figure 6.6). Despite this, the nearest distance from these patches 
(i.e. seed sources) in the ecological hub to the main patches covered by the class Gorse and/or Broom (i.e. deposit sites) is not suitable in all directions as distances range from $30 \mathrm{~m}$ (most suitable - from patch H) to 1607 m (most unsuitable - from patch G) (q.v. Figure 6.5 - top right).

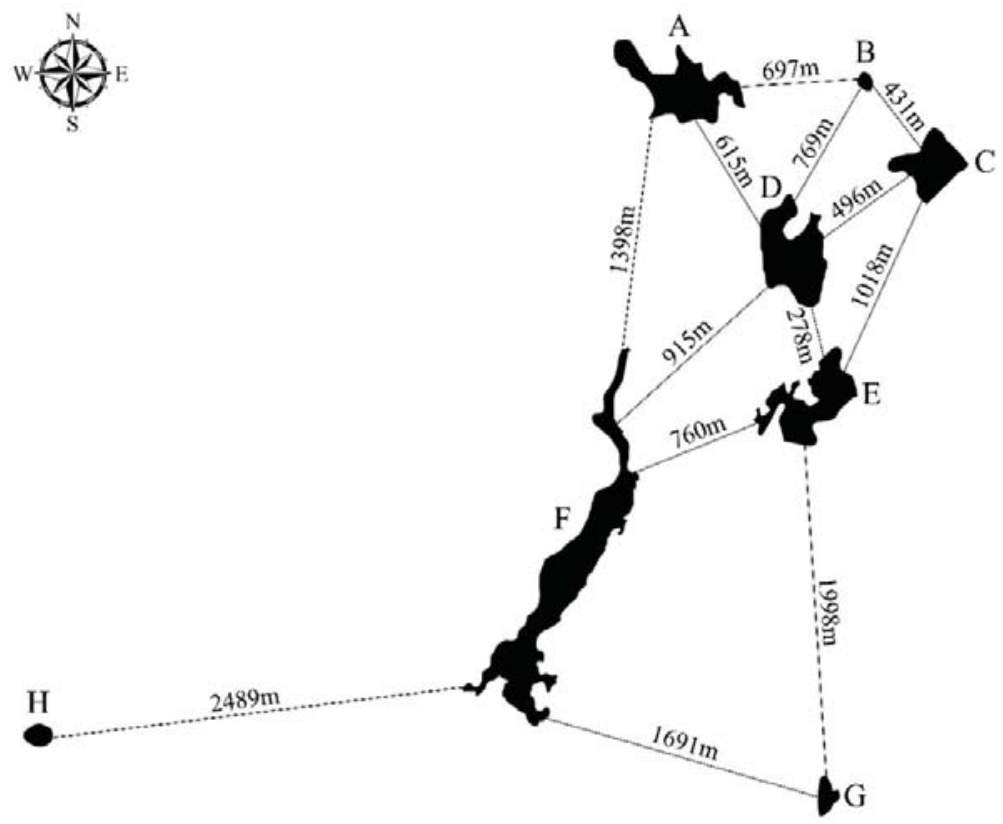

Figure 6.6. Nearest edge-to-edge distance between patches of indigenous forest in the ecological hub identified in the western part of the Wellington urban landscape (q.v. Figure 6.5) - map created based upon LCDB (Landcare Research, 2015).

\subsection{DISCUSION}

\subsubsection{Natural regeneration in urban landscapes: barriers and opportunities}

Results show that the Wellington urban landscape is potentially capable of increased rates of natural regeneration because long-term ecological data pertaining to land cover is relatively rich and enables an understanding of the potential opportunities available. There are also areas covered by the class Gorse and/or Broom that can function as nurse plants to facilitate the process of natural regeneration. Gorse and broom, along with some other exotic plants, as discussed by Ewel and Putz (2004), can be an integral part of landscape restoration in New Zealand as well as in other cases in the Southern Hemisphere.

In addition, rainfall and topography, as two key factors, provide suitable conditions for natural regeneration in the study area. The distance from seed source to the deposit site, however, is greater than typically required for natural regeneration (q.v. Figures 6.5 and 6.6). While landscape fragmentation may increase the distance between seed sources and areas capable of natural 
regeneration (i.e. deposit sites), a catalyst factor - that is the presence of a range of influential seed dispersers - may assist dispersal of indigenous tree species seeds to suitable places. In this research it was indicated that the ecological hub in the western part of the study area consists of a fragmented but relatively clustered pattern of patches of vegetation covered by the class Indigenous Forest. The minimum and maximum distances between these patches are $278(\mathrm{D}-\mathrm{E})$ and $2489 \mathrm{~m}(\mathrm{~F}-\mathrm{H})$, respectively. From patch $\mathrm{A}, \mathrm{F}, \mathrm{G}$, and $\mathrm{H}$, the nearest edge-to-edge distances to the main patches of gorse and broom are 99, 559, 1607, and 30 m, respectively (Figure 6.6, top right; cf. Figure 6.5). Therefore, kererū could theoretically transport seeds from these seed sources to patches covered by the class Gorse and Broom (cf. Kelly et al., 2010; Powlesland et al., 2011; Wotton and Kelly, 2012). However, from some seed sources (e.g. F and G), additional stepping stones may be required to facilitate species movement and thereby, the process of gene flows. Kererū stop between each flight for an average of $32 \mathrm{~min}$ and a maximum of $315 \mathrm{~min}$ (Wotton and Kelly, 2012). For landscape architecture, this means that kererū stay for relatively long periods in their habitats. For this reason, the number of patches of vegetation, either as habitat or stepping stone, should be managed in a way that ensures the species of kererū functions as a seed disperser. In the ecological hub, there is a gap between the eight patches of indigenous forest and patches of gorse and broom. This gap, however, is bridged by relatively large areas covered by the class Broadleaved Indigenous Hardwoods (Figure 6.5 - right bottom). Patches covered by this class fulfil therefore the role of stepping stones to provide functional connectivity between seed sources and deposit sites; however these patches cannot be regarded as seed sources because the large-fruited flora are almost completely absent in this class (q.v. Appendix V).

This research also shows where these stepping stones should be spatially located to overcome spatial disconnectivity and facilitate the ecological process of gene flow through providing enough connectivity and proximity between patches of ecological importance (q.v. Figures 6.5 and 6.6). An important implication of this for landscape architecture and land use planning practitioners is that the allocation of land for anthropogenic development must be carefully planned to avoid land cover change in areas capable of serving as seed source, deposit site, or stepping stone particularly within the ecological hub. In addition, wherever possible, private land owners within the ecological hub should be encouraged to vegetate and/or re-vegetate an appropriate proportion of their properties with indigenous flora, specifically large-fruited plant species such as tawa (Beilschmiediatawa) and puriri (Vitexlucens), to provide additional seed source in stepping stones for seed dispersers. 
In line with spatial planning for natural regeneration, informed human interventions may also be necessary to support this process. For example, patches of kanuka (Kunzea ericoides) and manuka (Leptospermum scoparium) in gorse-dominated landscapes should be manually established where possible to support effective regeneration, because they provide desirable shade for seedlings and protect from wind disturbance (Sullivan et al., 2007). There are also barriers that may marginalise natural regeneration mechanisms. For example, the seeds of gorse remain in the soil and can be activated even after long periods of time when the right conditions occur (Williams, 2011). This is important to note because the higher the weed seed bank longevity, the lower the likelihood of replacement by indigenous species. Among the plant species that previously dominated the study area in the pre-human period (Leathwick et al., 2004; Landcare Research, 2012; q.v. Appendix VI), and are still present (Dymond and Shepherd, 2004) in the eight patches of indigenous forest in the study area, tawa and puriri are large-fruited plant species that have seeds that can only be dispersed by kererū and in some cases tūî (cf. Kelly et al., 2010). Therefore, from a spatial perspective, it is crucial to provide suitable habitats as well as stepping stones for relatively large gaped seed dispersers in urban New Zealand (Figure 6.7). There is a need to provide suitable habitats for them that is characterised by favourable food sources, and there is also a need to ensure their habitats protect them from negative impacts imposed by urban development and climate change where possible (q.v. Chapter 3). Thus, results suggest that developing knowledge of the spatial ecology of kererū and tūi plays a vital role in ensuring the persistence of species-ecosystem mutualism and consequently the long-term ecological integrity of urban New Zealand, as a whole.

Compared with other common landscape restoration strategies such as re-vegetation of indigenous plant species or eradication of exotic plant species by human intervention, natural regeneration may be regarded as the most sustainable one owing to its inherent benefits (Davis and Meurk, 2001). For example, a field experiment on the effect of different management treatments of broom cover on germination, survival and growth of indigenous tree and shrub species revealed that chemical or mechanical control of invasive woody weeds (e.g. gorse and broom) tends not to be effective in landscape restoration practices, because such treatments are expensive and difficult and may adversely lead to disturbances in soil composition and micro-site conditions, whereas germination and survival rates of indigenous woody species under a living broom canopy are likely to be higher (Burrows et al., 2015). 


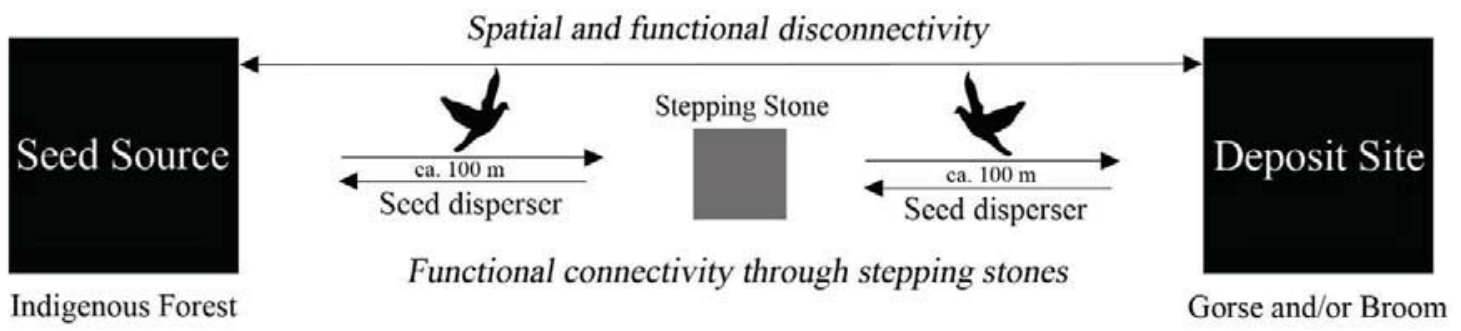

Figure 6.7. A conceptual spatially-explicit landscape pattern for providing functional connectivity for facilitating species movement and seed dispersal mechanisms between seed sources and deposit sites through stepping stones

\subsubsection{A four-step process for evidence-based natural regeneration}

The knowledge gained and arguments made in this research offer a new four-step process for research and practice in landscape architecture and land use planning for natural regeneration in the Southern Hemisphere to support a more sustainable spatial management approach that is based upon a range of necessary site-specific ecological data in urban landscapes. These data are in relation to: (1) the potential land-based opportunities for landscape restoration practices through natural regeneration including the ecological succession of some patches of vegetation under suitable conditions; (2) the spatial ecology of species playing pivotal roles in seed dispersal and pollination in relation to their habitat requirements; (3) climatic and environmental factors such as rainfall and topography affecting species and also targeted seed sources and nurse plants in deposit sites; and (4) connectivity and proximity between habitat patches through stepping stones that can facilitate species movement and gene flow across the landscape.

This four-step process (Figure 6.8) consists of four questions derived from landscape ecology science followed by four main steps designed to move towards an understanding of natural regeneration mechanisms in urban New Zealand. It provides a straightforward approach to natural regeneration in Southern Hemisphere urban landscapes ${ }^{52}$. Although the aim of this research was not to incorporate rainfall and temperature patterns into the process of spatial analysis in the GIS environment, rainfall and temperature have been included in this four-step process because they are thought to be key factors in a changing climate. The accumulation of carbon in the atmosphere leads to global warming (IPCC, 1995). This, in turn has given rise to changes in precipitation and

\footnotetext{
${ }^{52}$ It should be noted that not all urban landscapes in the Southern Hemisphere are at the same stage as the Wellington urban landscape, and not all countries equally invest in landscape restoration research and practice. Public environmental awareness and volunteer-centred activities, as two major factors in this process, differ among countries. Thus, without financial support and public environmental awareness the chance of success is very low.
} 
temperature patterns in different parts of the world (IPCC, 1995, 2007, 2014; q.v. Footnote 2). Any change in rainfall and temperature patterns causes multifarious impacts in the environment including the occurrence of rare or unprecedented heat waves, heavy storms, stronger lighting strikes, sudden flooding events, widespread inundation, and sea level rise (q.v. Section 1.1.3.3). As discussed in Chapter 6, rainfall and temperature play an important role in the process of landscape restoration in south-facing slopes covered by the class Gorse and/or Broom (q.v. Figure 6.4). Therefore, they should be considered as two determinant factors in this process. Fine-scale spatial data for these two determinant factors is needed in order to enable researchers to undertake overlay analysis at the urban scale in future research.

As indicated in New Zealand, research is in progress in all four steps of this framework to allow landscape architects and land use planners to make more informed spatial decisions to help facilitate natural regeneration in specific locations based upon sound knowledge. Data for the fourth step are the most undeveloped area compared to data required to complete the first three steps. The required data for all four steps are still lacking in many Southern Hemisphere countries and should be a research priority in the future. 
요

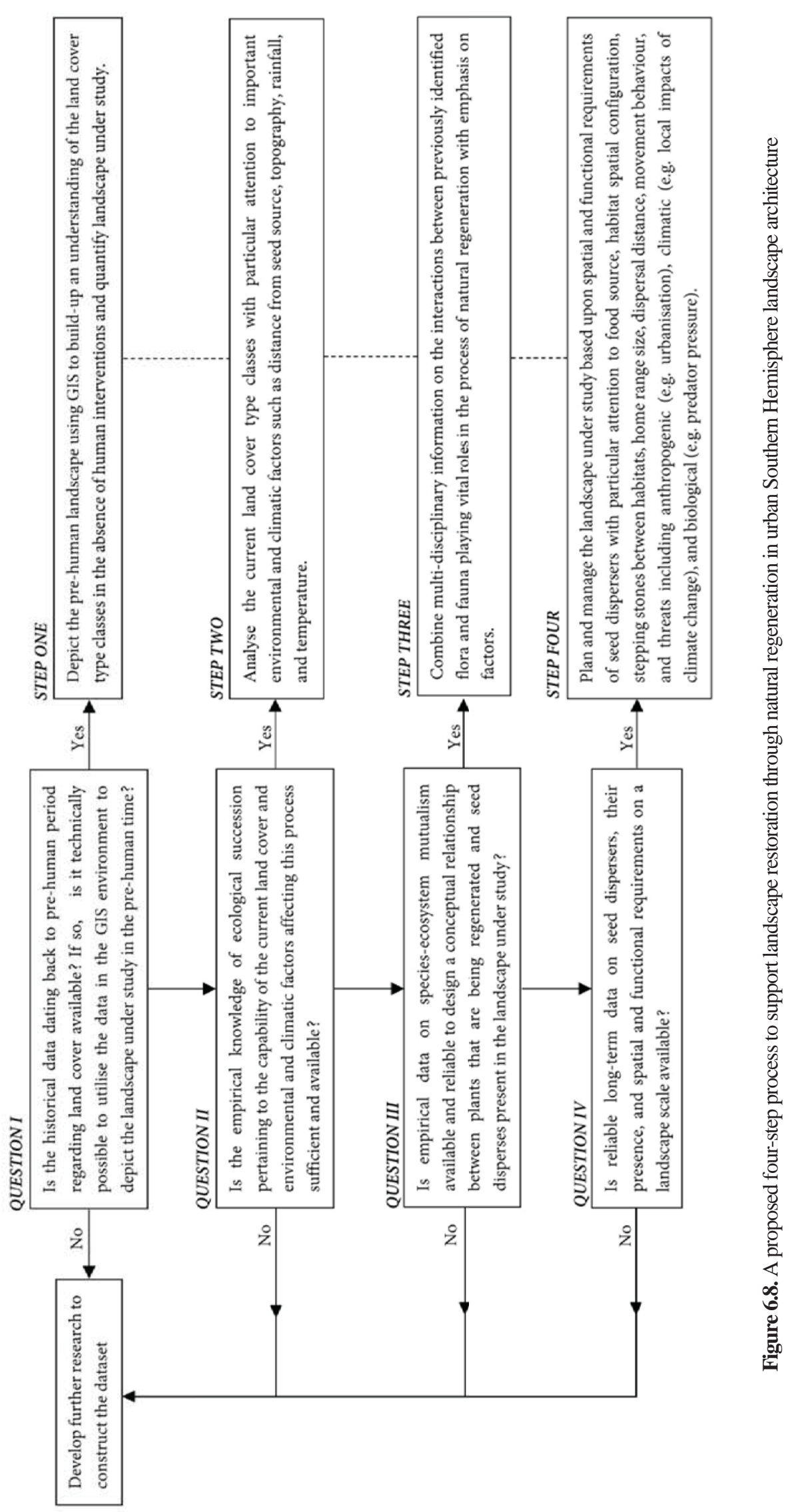




\subsection{CONCLUSION}

Natural regeneration in protected areas has been regarded as a key strategy for landscape restoration in some Southern Hemisphere countries (Veblen and Ashton, 1982; Wadt et al., 2008). Such practices should be extended to urban landscapes given that the multi-faceted pressures of urbanisation, climate change, and biodiversity loss in urban landscapes of the Southern Hemisphere are likely to give rise to further decline in the ecological integrity of ecosystems in this region. The research shows that knowledge of seed dispersal mechanisms and functional connectivity is essential to be incorporated into landscape architecture to provide a basis for informed decisions on the allocation of land use in urban landscapes.

With particular reference to this research, a key implication for the conservation of indigenous biodiversity in urban New Zealand is that kererū and tūī are species of particular ecological significance owing to their ability to disperse seeds of large-fruited indigenous trees and their relative abundance at the present time. Thus, suitable habitats, food sources, and stepping stones are required for the survival and long-term presence of these species. This goal could likely be achieved by considering the species' behaviours in space and time in relation to the current landscape pattern composition and configuration. This issue will be addressed and discussed in Chapter 7. To develop greater knowledge of natural regeneration in landscape architecture and land use planning in urban landscapes, four main questions should be addressed using a new four-step process as proposed in this research to provide a proper platform for informed decision-making on human land use activities and spatial management of landscapes in support of natural regeneration mechanisms. The use of GIS will help to ensure a higher level of accuracy.

Overall, the method and process followed in this part of the research can be transferred to other areas in New Zealand or the Southern Hemisphere to respond to the current indigenous biodiversity loss and simultaneously provide a basis for informed practices to support natural regeneration in urban landscapes. 


\section{CHAPTER 7}

\section{Study III: A class-level analysis of the spatial patterning of land cover classes}

\subsection{RESEARCH AIMS}

In Chapter 4, the spatial extent of land cover classes was quantified and measured in terms to indigenousness, land surface perviousness, and land cover heterogeneity. In Chapter 5, landform diversity was addressed in relation to the spatial extent of the two classes Indigenous Forest and Broadleaved Indigenous Hardwoods. In Chapter 6, potentials for landscape restoration through natural regeneration mechanisms were examined through the connectivity and proximity between seed sources and deposit sites as well as the ability of keystone species for seed dispersal. In this chapter, the spatial composition and configuration of land cover classes are scrutinised at the class level. The principal aims of this research are to:

(1) Quantify and measure the spatial composition and configuration of land cover classes in terms of patch size, edge density, connectivity and proximity, and shape complexity;

(2) Examine landscape pattern composition and configuration that has the potential to help safeguard indigenous avifauna against the local impacts of climate change, by undertaking a class-level spatial analysis of the Wellington urban landscape using GIS and FRAGSTATS.

Particular emphasis is placed on what is currently known about possible interactions between landscape patterns (i.e. the spatial composition and configuration of land cover classes in the study area) and ecological processes (i.e. the behaviours of avifauna in space and time).

\subsection{INTRODUCTION}

The spatial configuration of land cover classes, including shape and distance between habitat patches, influences the degree to which a landscape can potentially safeguard fauna against the adverse effects of climate change (McDonald, et al., 2008; Heller and Zavaleta, 2009). As the 
literature suggests (q.v. Chapters 2 and 3), the four components of landscape pattern patch size, edge density, connectivity and proximity, and shape complexity can be used as reliable indicators to quantify and measure every land cover class with regard to the spatial ecology of urban fauna, in order to examine if the spatial patterning of different patches of vegetation is capable of providing suitable conditions for them. The central question examined in this chapter is:

What are spatial opportunities and barriers for safeguarding indigenous avifauna against some impacts of climate change in terms of the spatial patterning of patches of vegetation distributed in urban landscapes and consequently, and what are the main implications of these opportunities and barriers for the persistence of urban avifauna under climate change?

Addressing this question will lead to a higher level of practical knowledge concerning the spatial design of land cover patterns based upon species behaviour in space and time.

\subsection{MATERIALS AND METHODS}

A class-level spatial analysis was performed to address possible interactions between landscape patterns and ecological processes at the class level using the Wellington urban landscape as the study area. The research was conducted in four parts: (1) a review of the relevant literature on spatial ecology of species of interest as well as possible impacts of climate change on them, (2) conducting semi-structured interviews with six subject-matter experts to discuss issues that cannot be found in the literature (q.v. Chapter 3), (3) mapping the study area using GIS, and (4) quantifying and measuring land cover patterns through a core set of landscape metrics calculated by FRAGSTATS v.4.2. The first two parts were conducted to provide a scientific basis for enabling more informed interpretations of the FRAGSTATS outputs (Figure 7.1). 


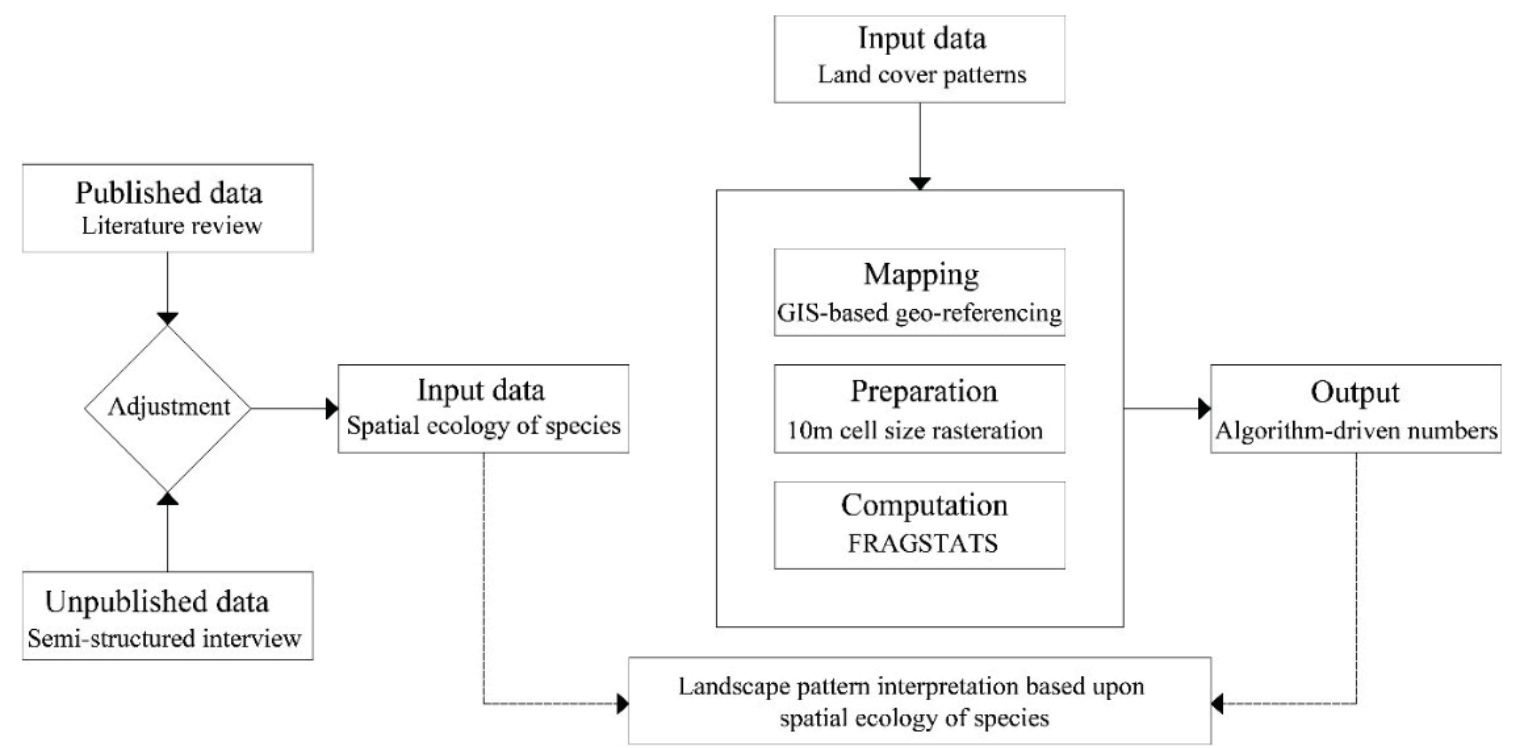

Figure 7.1. The relationship between spatial and non-spatial data and stages undertaken in the research

\subsubsection{Consultation with subject-matter scholars}

In Study II (q.v. Chapter 3), specific data not currently available in the literature was gathered for this part of the research. In particular, the list of keystone species, initially derived from a literature review, was addressed and finalised. The possible impacts imposed by climate change on the selected keystone species and their habitats were discussed, and required inputs for running FRAGSTATS (i.e. edge depth, search radius, and threshold distance) were identified and confirmed with particular reference to the spatial ecology of the selected keystone species. Ultimately, a consensus on three categories was observed:

(1) Kererū, tūī, bellbird, and hihi are among the most important keystone species in the study area that support vital ecosystem services such as pollination and seed dispersal mechanisms;

(2) Changes in wind and temperature patterns are likely to have the most detrimental impacts on the chosen species, especially along edges of habitat patches;

(3) An edge depth of 50m and a search radius/threshold distance of $100 \mathrm{~m}$ were confirmed to be suitable for the spatial analysis of the current landscape patterns. 
The value of edge depth represents the degree to which environmental variables affect a habitat patch along its edges towards the core area. The concept of search radius is applied to test the possibility of species movement and seed dispersal success (q.v. Section 3.3.1.6).

\subsubsection{Avifauna and climate change impacts}

The spatial ecology, foraging behaviour and habitat requirements of four New Zealand endemic avifauna, namely kererū, tūī, bellbird, and hihi, were taken into consideration to provide a scientific platform for landscape pattern interpretation in the case study area. As discussed in Study II (q.v. Chapter 3), these are the most important keystone species in urban New Zealand (cf. Payton et al., $2002)^{53}$. There is sufficient evidence to show that the presence of these species plays a pivotal role in seed dispersal mechanisms as well as pollination processes in urban New Zealand (e.g. Clout and Hay, 1989; Anderson et al., 2006; Kelly et al., 2010; Anderson et al., 2011; Wyman and Kelly, 2017). This, in turn, can contribute to natural regeneration (q.v. Chapter 6). More specifically, kererū and tūī are the only remaining seed dispersers of large-fruited indigenous trees in New Zealand (Wyman and Kelly, 2017). Recent surveys show that tūi, bellbird, and kererū are respectively the $5^{\text {th }}, 11^{\text {th }}$, and $14^{\text {th }}$ most widely occurring species in New Zealand gardens, but only the $25^{\text {th }}, 20^{\text {th }}$, and $27^{\text {th }}$ most widely occurring in $10 \mathrm{~km}$ grid squares in the country (Spurr, 2012). Therefore, these species are still well represented in urban landscapes, meaning that the absence or poor presence of these species is likely to adversely affect the ecological integrity of urban ecosystems.

A middle of the range estimation suggests that Wellington is very likely to experience a $2 \%$ to $5 \%$ increase in the frequency of extreme winds in winter and a $0.9^{\circ} \mathrm{C}$ to $2.1^{\circ} \mathrm{C}$ increase in annual average temperature by 2040 and 2090 respectively compared to 1990 (Ministry for the Environment, 2014; q.v. Section 1.4.2). Regardless of a wide range of impacts attributed to the changing climate (McKinney, 2002; Parmesan, 2006) including species’ phenological and physiological changes (Rosenzweig et al., 2007; Chauvenet et al., 2013), as well as the outbreak and spread of diseases amongst the chosen avifauna (Baillie and Brunton, 2011; Howe et al. 2012; Niebuhr, 2016), scientific evidence shows that the primary habitats of the selected species will be affected through two main mechanisms in a changing climate:

\footnotetext{
53 As advised in Study II (q.v. Chapter 3), forest gecko and Wellington green gecko are also regarded as keystone species in the Wellington urban landscape. Nevertheless, the home range of these reptiles cannot be studied at the class-level analysis. The potential contribution of New Zealand reptiles to pollination and seed dispersal should be studied at the patch-level. Therefore, the spatial ecology of these species cannot be currently regarded as a lens to examine the capability of landscape pattern for safeguarding the overall level of biodiversity at the class level.
} 
(1) Environmental disturbance and habitat loss along edges of habitat patches due to wind penetration into habitat core area increasing edge effects (Yang and Mitchell, 1984; DaviesColley et al, 2000);

(2) Increased predator pressures due to the mast seeding phenomenon caused by rising temperatures mainly along edges of habitat patches (McGlone and Walker, 2011; Christie, 2014; q.v. Table 1.5).

In terms of climate change, changes in wind and temperature patterns were identified as the most detrimental climatic factors affecting the chosen species and their habitats in the study area (q.v. Chapter 3). In addition, climate change impacts can lead to the loss of primary habitats, through even more complex and ruinous processes. For example, the exotic pest mammal brushtail possum benefits from rising temperatures and is responsible for increasing the mortality rate of canopy trees of the indigenous tree northern rātā (Metrosiderosrobusta) in Wellington (Cowan et al. 1997; q.v. Section 1.1.5). This is one of New Zealand's endemic and rare plant species of ecological importance, and it provides primary habitats and a key source of food for indigenous avifauna (King et al., 2015).

\subsubsection{Datasets}

LCDB (q.v. Section 4.3) was utilised to generate a land cover map of the Wellington urban landscape using Arc Map v.10.4.1 (Landcare Research, 2015). As shown in Chapter 4, the study area consists of sixteen land cover classes. Areas of indigenous and exotic patches of vegetation collectively cover 8043.7 ha (59.53\%) of the study area. The rest is covered by the three classes of Built-up Area, Transport Infrastructure, and Surface Mine or Dump, as well as three non-vegetative ones (Sand or Gravel; Lake or Pond; and Gravel or Rock). Particular emphasis was placed on ten land cover classes of indigenous and exotic flora that support different forms of plant life (q.v. Appendix V). Indigenous and exotic classes cumulatively cover 3258 ha (24.11\%) and 4785.7 ha (35.42\%) of the study area, respectively (q.v. Table 4.1). Using this map based upon LCDB, a rasterised map with a cell size of $5 \mathrm{~m}$ was defined in the GIS environment to prepare the required input for landscape computation in FRAGSTATS v.4.2 (McGarigal et al., 2012).

\subsubsection{Selection of landscape metrics}

Landscape metrics provide eco-spatial analysts with strong tools for interpreting landscape pattern-process relationships (Botequilha Leitao et al, 2006; McGarigal et al., 2012). Some landscape metrics may be more suitable for scrutinising potential interactions between patterns and processes 
depending on the scale of study, landscape heterogeneity and the ecological traits and niches of species of interest (Gustafson, 1998; Kupfer, 2012). With particular emphasis on the ecological traits and niches of the selected species (q.v. Section 7.3.5), ultimately fourteen landscape metrics were selected through a comprehensive review of a considerable number of key sources including Turner, 1989; Gustafson, 1998; Turner et al., 2001; Botequilha Leitao and Ahern, 2002; Cook, 2002; Neel et al., 2004; Seto and Fragkias, 2005; Cushman et al., 2008; Schindler et al 2013; Uuemaa et al., 2013; Wang et al., 2014; Walz et al., 2016; Frank and Walz, 2017 (Table 7.1). This core set of landscape metrics was finalised after checking a larger number of landscape metrics for collinearity (Corry and Nassauer, 2005; Botequilha Leitao et al., 2006; McGarigal, 2015). The aim was to find the most relevant landscape metrics and study their inherent applications and limitations. Although numerous landscape metrics are available in the literature (McGarigal, 2015), it has been widely recommended by researchers to select a limited number of these depending on the aim, scale, and nature of study as well as species of interest (Botequilha Leitao and Ahern, 2002; McGarigal et al., 2012; McGarigal, 2015). Each landscape metric can only measure some aspects of landscape patterns. At the same time, many measure a similar or identical aspect of landscape patterns (McGarigal et al., 2012). Thus, irrelevant landscape metrics were omitted with respect to the aims of this study (q.v. Section 7.1) as well as the spatial ecology of the selected species (q.v. Section 7.3.5). In comparison with patch- and landscape-level landscape metrics, class-level ones are the most relevant and appropriate indices for a more in-depth analysis of landscape patterns (Botequilha Leitao et al., 2006). Given this, landscape metrics selected for this research are those that measure the composition and configuration of land cover patterns at the class level. According to the literature cited above, these are among the most powerful class-level tools for measuring patch size, edge density, connectivity and proximity, and shape complexity. Nevertheless, landscape metrics should not be regarded as a panacea to patternprocess issues and questions (Botequilha Leitao and Ahern, 2002), because FRAGSTATS and landscape metrics are only as good as the proficiency and expertise of the landscape pattern analyst (McGarigal, 2015).

To measure the aggregate properties of the patches belonging to an individual class, the areaweighted mean (_AM) of six metrics was computed to include the ecological importance of patch size into the analysis. According to McGarigal (2015), the area-weighted mean ranks each class by its proportional area representation in order to provide first- and second-order statistical summaries of the patch metrics for each class. It can compound essential information about the relative importance of each patch in relation to patch size (Botequilha Leitao et al., 2006). Total Class Area (CA), Percentage of Landscape (PLAND), Number of Patches (NP), Mean Patch Area (AREA_MN), Edge Density 
(ED), and Area-weighted Mean Radius of Gyration (GYRATE_AM) were used to compute structural characteristics of the landscape under study. Total Core Area (TCA), Core Area Percentage of Landscape (CPLAND), Area-weighted Core Area Index (CORE_AM), and Area-weighted Mean Core Area Index (CAI_AM) were used to reveal detailed information pertaining to core areas potentially serving as interior habitats. Area-weighted Mean Proximity Index (PROX_AM) and Areaweighted Mean Euclidean Nearest Neighbor Distance (ENN_AM) were used to quantify and measure isolation and proximity attributes of the classes. PROX_AM has been suggested as a useful measure to address the suitability of wildlife habitats in relation to species' home range size (Botequilha Leitao et al., 2006). Finally, Patch Cohesion Index (COHESION) and Connectance Index (CONNECT) were used to quantify landscape connectivity at the class level. COHESION is strongly correlated with dispersal success of fauna and therefore can be a strong indicator of connectivity. A detailed description of the selected metrics, including information on their applications in landscape pattern analysis, can be found in McGarigal and Marks (1995), McGarigal et al. (2012), Table 7.1 and Appendix VII. An edge depth of $50 \mathrm{~m}$ was used to compute Core Area metrics. This threshold has been suggested as one that will avoid edge effects in a New Zealand context (Yang and Mitchell; 1984; Davies-Colley et al., 2000; Meurk and Hall, 2006; Meurk et al., 2016). This is in agreement with many international studies including Murcia (1995) and Port Stephens Council (2002). In addition, a search radius of $100 \mathrm{~m}$ was applied to compute Isolation/Proximity and Connectivity metrics in FRAGSTATS. Sullivan et al. (2009) state the minimum distance between patches of vegetation should be less than $100 \mathrm{~m}$ to provide suitable conditions for seed dispersal by New Zealand avifauna. The same has been proposed in other studies (Meurk and Hall, 2006; Meurk et al., 2016). This is similar to a wide range of empirical research on the ability of New Zealand avifauna to disperse the seeds of indigenous trees (q.v. Sections 6.2.1 and 6.2.2). Feedback received from the six subject-matter experts in the course of Study II (q.v. Section 3.3.1.6) also supported these thresholds (i.e. edge depth and search radius) (Figure 7.2). 


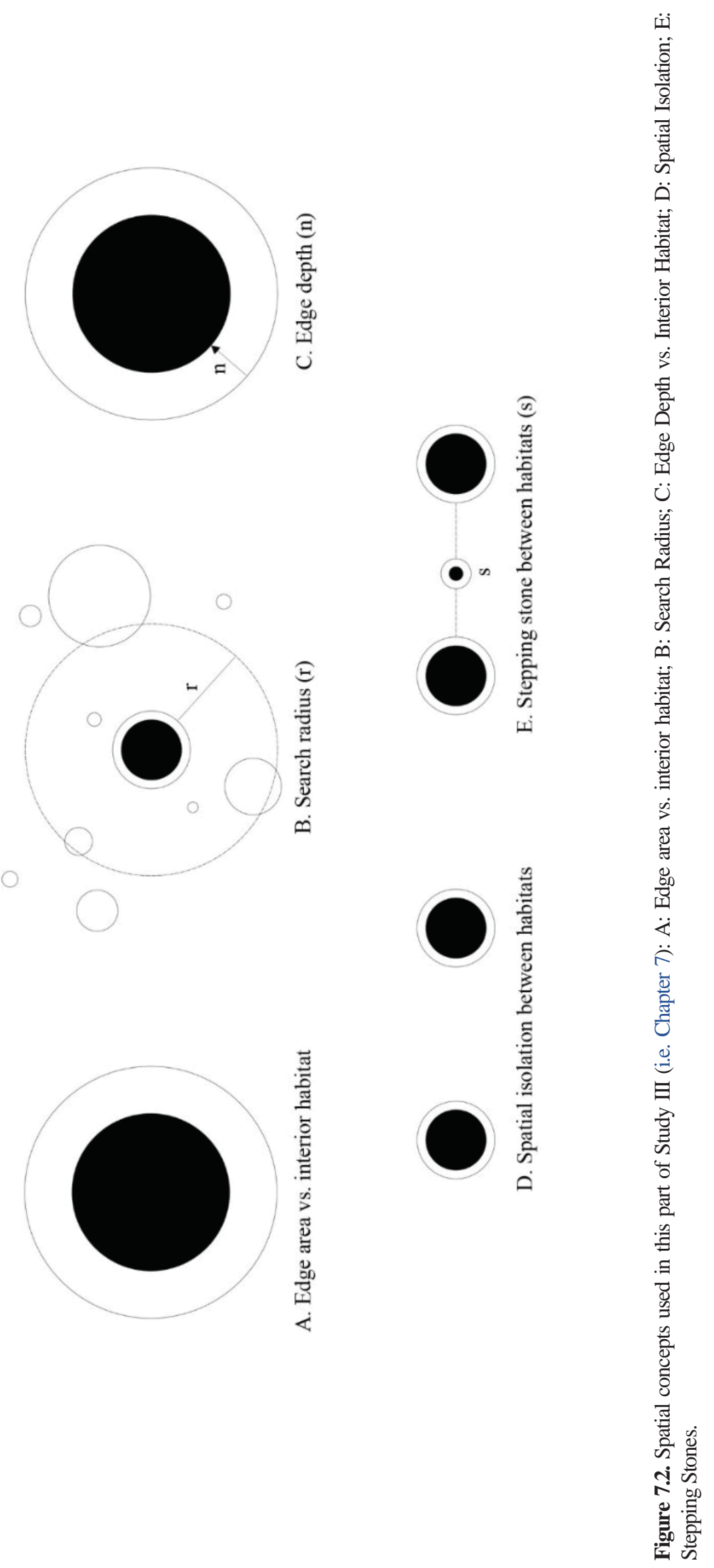


I

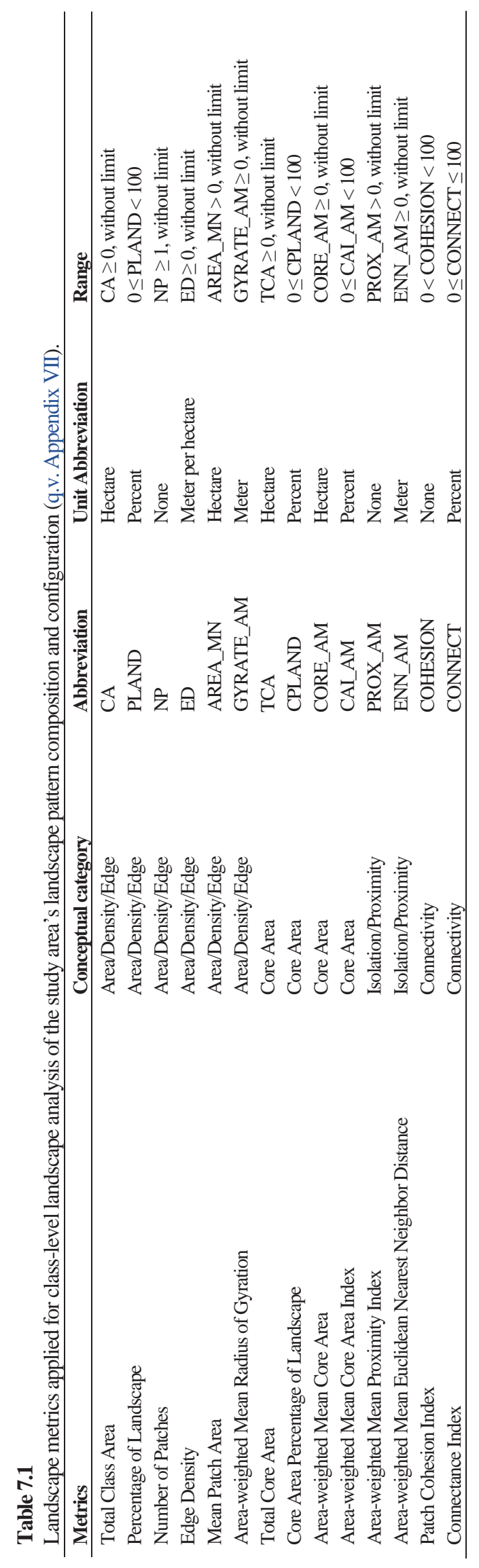




\subsubsection{Spatial ecology of the selected species}

Behaviours of the selected species in space and time were studied by reviewing a wide range of empirical research including Bell, 1996; Mander et al., 1998; Baker, 1999; Taylor et al., 2005; Campbell, 2006; Stevens, 2006; Wotton, 2007; Sullivan et al., 2009; Innes et al., 2010; Powlesland et al., 2011; Barbaro et al., 2012; Wotton and Kelly, 2012; Innes et al., 2015a and 2015b; Wyman and Kelly, 2017. The publications reviewed are of the most reliable published data in New Zealand providing specific empirical information on the ecological traits and niches of species of interest for this research. In addition, these issues were discussed in detail with six subject-matter experts through semi-structured interviews in Study II to increase the information accuracy level (q.v. Chapter 3). Taking a precautionary approach, those ecological traits and niches for which there was not a consensus were not taken into account. Taken together, the final output suggests that the following information reveals the most important aspects of ecological processes in relation to landscape patterns, and therefore can be regarded as a reliable platform for interpreting landscape patterns in this research:

(1) Areas covered by the class Indigenous Forest are the primary habitats of the selected species;

(2) Interior habitats are preferable for the selected species than edge areas;

(3) Predation by introduced mammals is the primary cause of the population loss of the selected species;

(4) The presence of exotic and invasive flora and fauna along edges of habitat patches is greater, and predator pressure on the selected species is likely to be consequently higher;

(5) In the absence of sufficient food sources year-round, kererū, tūī, and bellbird are forced to fly several kilometres in search of food sources, specifically during winter;

(6) Kererū is a sedentary species and hihi is confined to pest-free wildlife sanctuaries, whereas bellbird and tūī are relatively more dynamic in urban landscapes;

(7) Tūî is more resilient than other species due to its mobility, and aggressive and opportunistic behaviour in foraging; 
(8) Exotic forests may be seasonally used by kererū, tūī, and bellbird to compensate for food source scarcity;

(9) The selected species can disperse the seeds of indigenous trees up to $100 \mathrm{~m}$ from the seed source;

(10) Due to its relatively large gape size, kererū is the only species that can disperse the seeds of large-fruited trees endemic to New Zealand;

(11) Bellbird, hihi, kererū, and tūī will suffer from rising temperatures as well as the occurrence of intensified winds in the coming decades; however, the magnitude of impacts is still largely unknown.

This set of species information was used as a lens for interpreting spatial information in the study area with particular attention to the local impacts of climate change on the primary habitats of the selected species.

\subsection{RESULTS}

Detailed information on the values of the fourteen landscape metrics computed for the ten land cover classes was summarised and tabulated (Table 7.2 and Figure 7.3). Results are classified and presented in terms of the four main categories of landscape metrics: (1) Area/Density/Edge metrics, (2) Core Area metrics, (3) Isolation/Proximity metrics, and (4) Connectivity metrics. 
q

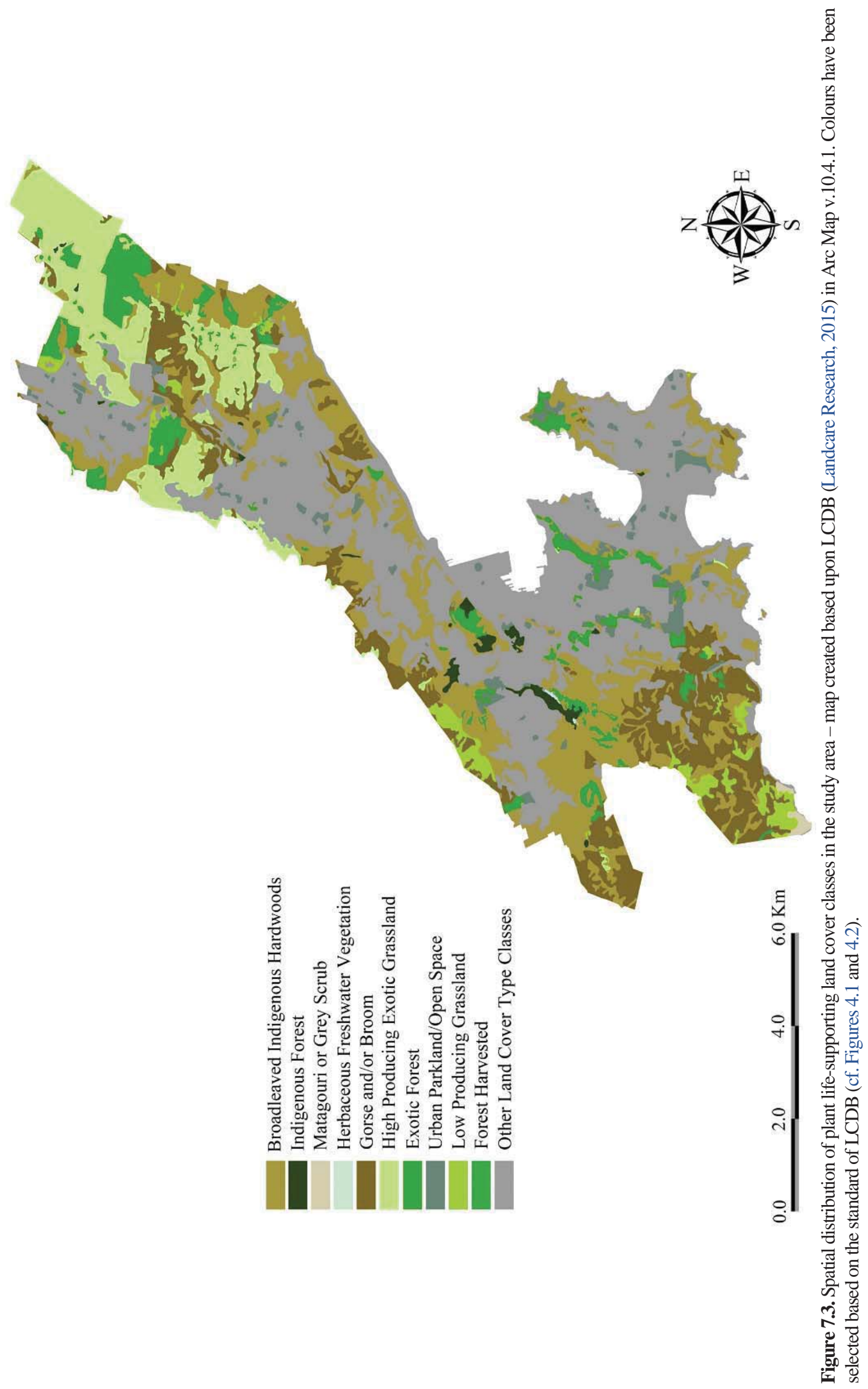


专

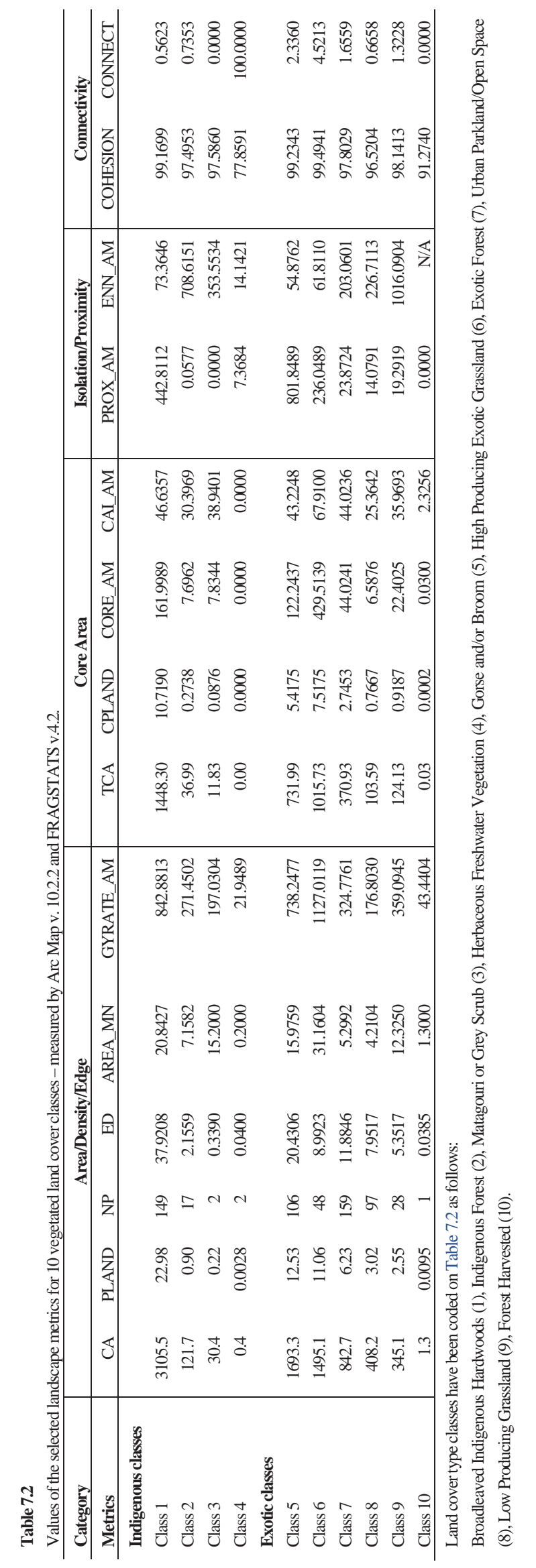




\subsubsection{Area/Density/Edge metrics}

Values of Area/Density/Edge metrics are depicted in Figure 7.4. Broadleaved Indigenous Hardwoods (3105.5 ha), Gorse and/or Broom (1693.3 ha), High Producing Exotic Grassland (1495.1 ha), Exotic Forest (842.7 ha), and Urban Parkland/Open Space (408.2 ha) have greater values in CA. In contrast, the lowest values of CA are observed for Low Producing Grassland (345.1 ha), Indigenous Forest (121.7 ha), Matagouri or Grey Scrub (30.4 ha), Forest Harvested (1.3 ha), and Herbaceous Freshwater Vegetation (0.4 ha).
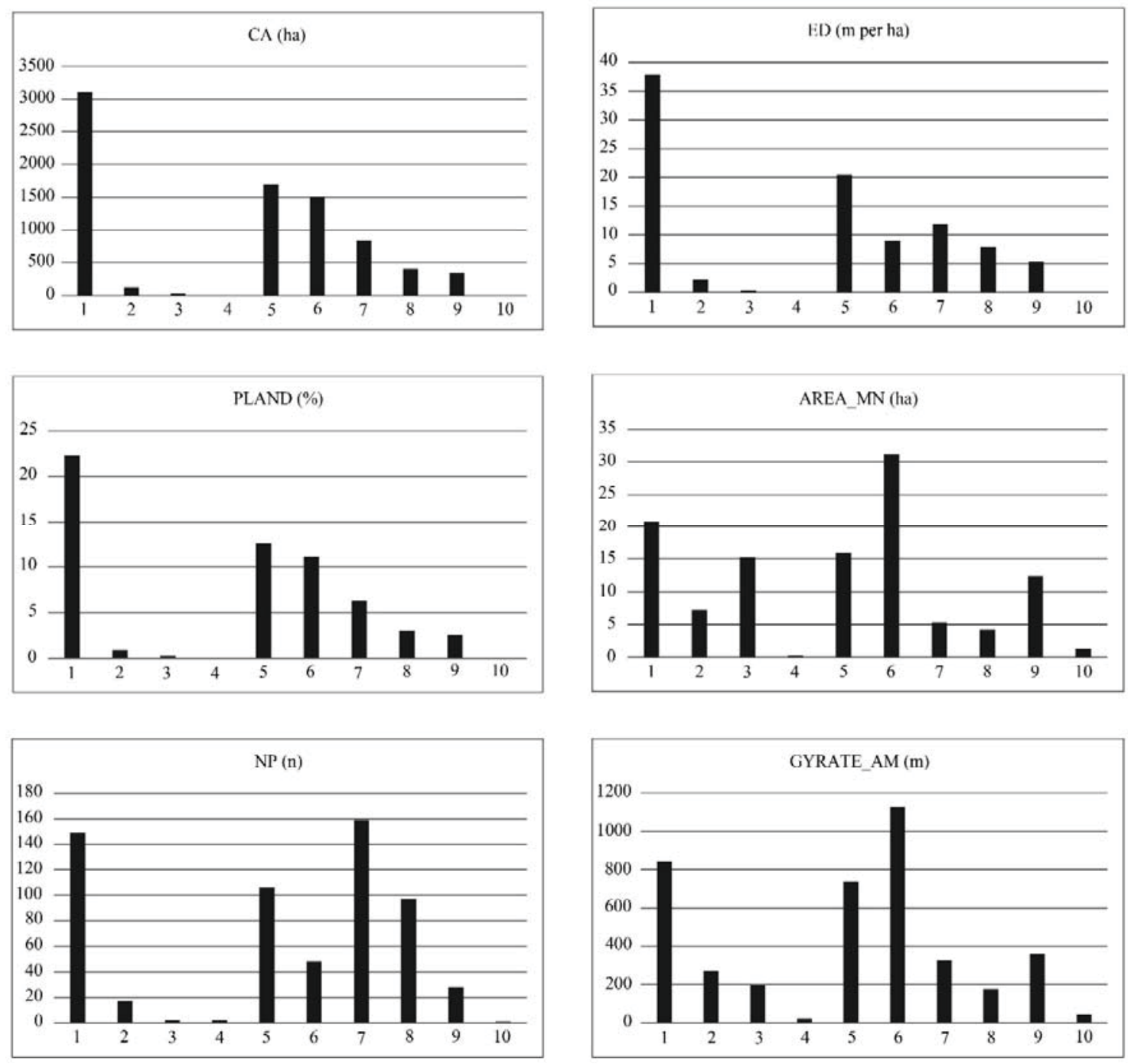

Figure 7.4. Area/Density/Edge metrics calculated for the 10 land cover classes (cf. Table 7.2).

Broadleaved Indigenous Hardwoods (1), Indigenous Forest (2), Matagouri or Grey Scrub (3), Herbaceous Freshwater Vegetation (4), Gorse and/or Broom (5), High Producing Exotic Grassland (6), Exotic Forest (7), Urban Parkland/Open Space (8), Low Producing Grassland (9), Forest Harvested (10). 
According to PLAND values, more than one-fifth of the study area is dominated by the class Indigenous Broadleaved Hardwoods (22.98\%). These are followed in decreasing extent by five exotic classes: Gorse and/or Broom (12.53\%), High Producing Exotic Grassland (11.06\%), Exotic Forest (6.23\%), Urban Parkland/Open Space (3.02\%), and Low Producing Grassland (2.55\%). The class Indigenous Forest dominates only $0.9 \%$ of the study area. Cumulative coverage of the other three classes is not considerable (0.3\%).

In terms of NP, the first six classes are ranked as Exotic Forest (159), Broadleaved Indigenous Hardwoods (149), Gorse and/or Broom (106), Urban Parkland/Open Space (97), High Producing Exotic Grassland (48), Low Producing Grassland (28). The class Indigenous Forest consists of 17 patches.

Edge density was measured to quantify each class in terms of the availability of edge habitats. In general, edge areas are more exposed to extreme weather events, higher predator pressure, and negative effects of human activities (Murcia, 1995; Fernández-juricic, 2001). This, consequently, can affect the richness and presence of the selected avifauna along edges of habitat patches.

The classes Broadleaved Indigenous Hardwoods and Gorse and/or Broom have the highest value of ED, to a high degree of certainty due to having greater values of CA and PLAND. Although Exotic Forest's CA and PLAND values are less than that of High Producing Exotic Grassland, a higher ED was calculated for the class Exotic Forest (11.88 m per ha) indicating that patches of exotic forest are spatially more dispersed across the study area.

AREA_MN’s highest quantity was recorded for the class High Producing Exotic Grassland (31.16 ha). This figure is important because the values of CA and PLAND for this class are about half of that of the class Broadleaved Indigenous Hardwoods. In addition, the number of patches covered by this class is relatively low (48). Thus, this value indicates that the class consists of larger patches in comparison with other classes.

Patch extensiveness was also measured at the class level applying GYRATE_AM. By definition, the value of GYRATE_AM shows the average distance an individual species can move in the landscape 'while remaining within a patch from a random starting point and moving in a random direction' (Botequilha Leitao et al., 2006). This metric is positively correlated with the degree to which an individual patch is extensive in a given landscape. According to the FRAGSTATS outputs, the highest value of GYRATE_AM belongs to High Producing Exotic Grassland (1127.01 m). The 
second and third highest values were recorded for the classes Indigenous Broadleaved Hardwoods (842.88 $\mathrm{m})$ and Gorse and/or Broom (738.24 m). While the total area of the class Exotic Forest is about seven times larger than that of the class Indigenous Forest, the latter class has a relatively high value of GYRATE_AM (324.77 m vs. $271.45 \mathrm{~m}$ ) revealing that the class Indigenous Forest has the greater potential to provide spatial continuity within a single patch of the same class.

\subsubsection{Core Area metrics}

Values of Core Area metrics have been depicted in Figure 7.5. Of 121.7 ha of the class Indigenous Forest, 36.99 ha can be used by the selected avifauna as interior habitats. This quantity is equivalent to $30.39 \%$ of the total class, and only $0.27 \%$ of the study area revealing more than two-third (84.71 ha) of the total class area is theoretically prone to edge effects, and therefore may not be suitable for safeguarding species from the direct and indirect impacts of climate change. In addition, while 22.98\% of the study area is covered by the class Broadleaved Indigenous Hardwoods, less than half (1448.30 ha) of the total class area was identified as core area. The rest of the class is therefore susceptible to edge effects. A similar situation is observed for the class Gorse and/or Broom (TCA = 731.99 ha). This class is believed to be the most important land cover class for initiating ecological succession towards indigenous forests (Davis and Meurk, 2001; Williams, 2011; Wotton and McAlpine, 2013).

The computed value of CORE_AM for the class High Producing Exotic Grassland is considerably greater than those of the classes Broadleaved Indigenous Hardwoods and Gorse and/or Broom (429.51, 161.99, and 122.24 ha, respectively). A possible explanation of this for the class High Producing Exotic Grassland can be because of lower and higher values of respectively NP and CA, compared to other classes. Indigenous Forest, however, has a relatively lower value in this metric (7.69 ha). This is very close to those of the classes Matagouri or Grey Scrub (7.83 ha) and Urban Parkland/Open Space (6.58 ha) despite the fact that these classes are completely different in terms of CA. This shows the importance of patch shape in landscape pattern analysis, as previously noted by others (Forman, 1995; Dramstad et al, 1996; Forman and Collinge, 1997).

To increase the accuracy of landscape pattern analysis, CAI_AM was also computed for each class. This metric shows the percentage of patches of vegetation in an individual class that is comprised of core area (McGarigal et al., 2012). This metric is thought to be one of the most powerful class-level metrics for measuring landscape fragmentation (Kupfer, 2012; Wang et al., 2014). Values of CAI_AM calculated for all classes, except for High Producing Exotic Grassland (67.91\%), are below 
50\%. More specifically, only 30.39\% of areas covered by the class Indigenous Forest are core areas unlikely to be prone to edge effects.
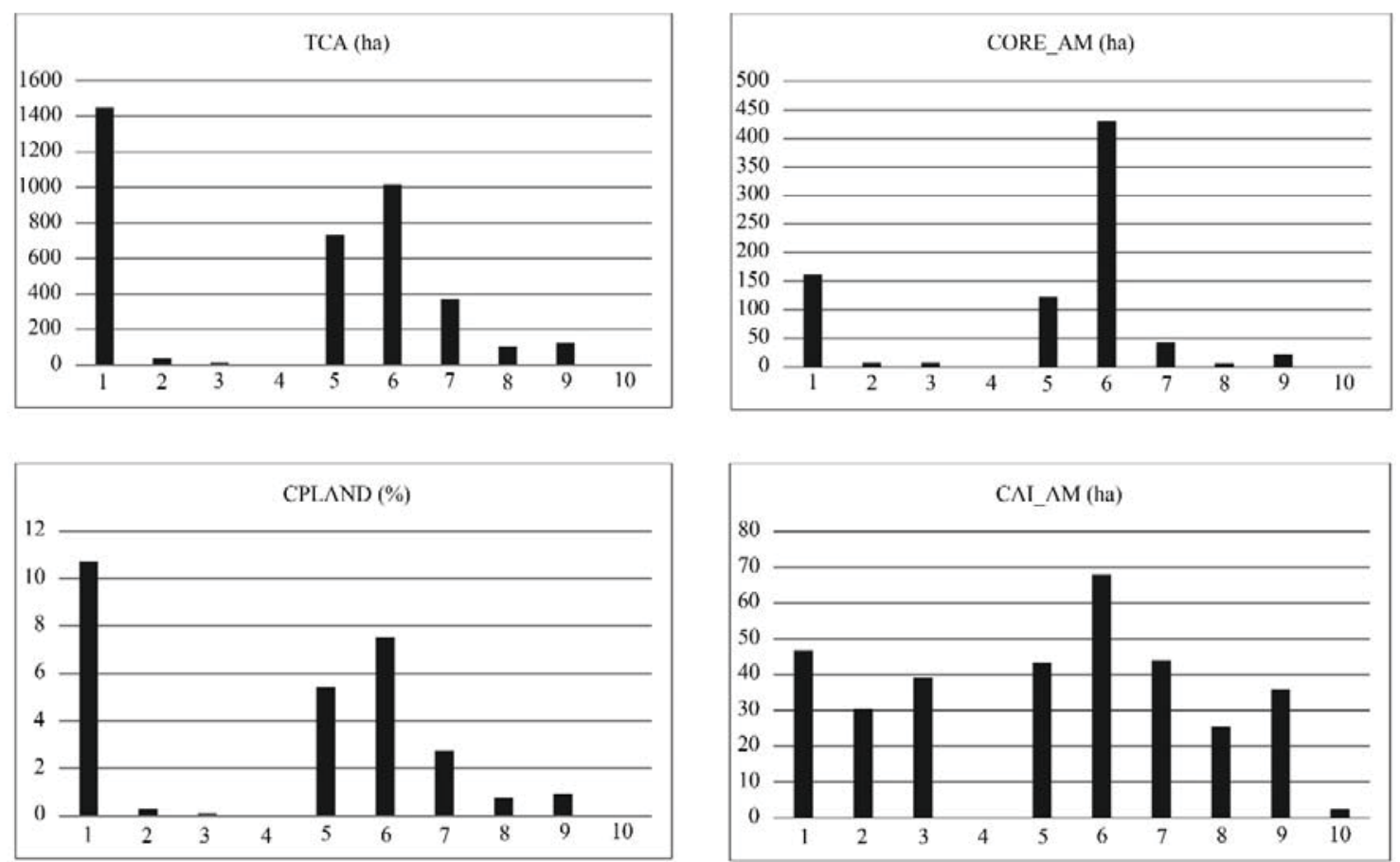

Figure 7.5. Core metrics calculated for the 10 land cover classes (cf. Table 7.2).

Broadleaved Indigenous Hardwoods (1), Indigenous Forest (2), Matagouri or Grey Scrub (3), Herbaceous Freshwater Vegetation (4), Gorse and/or Broom (5), High Producing Exotic Grassland (6), Exotic Forest (7), Urban Parkland/Open Space (8), Low Producing Grassland (9), Forest Harvested (10).

\subsubsection{Isolation/Proximity metrics}

Values of Isolation/Proximity metrics have been depicted in Figure 7.6. The highest and lowest values of PROX_AM were respectively recorded for the classes Gorse and/or Broom (801.84) and Indigenous Forest (0.05). Values of this metric for the classes Broadleaved Indigenous Hardwoods (442.81) and High Producing Exotic Grassland (236.04) are also relatively high. Low values were calculated for other classes. More specifically, two classes Matagouri or Grey Scrub and Forest Harvested have PROX_AM values of zero. Aside from the class Low Producing Grassland, with an ENN_AM value higher than $1000 \mathrm{~m}$, other classes have relatively lower values in this metric. The ENN_AM value computed for the class Indigenous Forest underscores a relatively high degree of isolation among patches of this class $(708.61 \mathrm{~m})$, whereas the value of ENN_AM for the class Broadleaved Indigenous Hardwoods is low (73.36 m) showing a higher degree of proximity between patches covered by this class. Values of the two classes Exotic Forest and Urban Parkland/Open Space in this metric are relatively similar (203.06 m and $226.71 \mathrm{~m}$ ). Proximity between patches of the 
class Gorse and/or Broom is also higher than other classes $(54.87 \mathrm{~m})$. Although this metric was calculated for the class Herbaceous Freshwater Vegetation as $14.14 \mathrm{~m}$, this figure does not necessarily represent close proximity between patches of herbaceous freshwater vegetation given that only 2 were identified in the study area ( $\mathrm{NP}=2$ ).

\subsubsection{Connectivity metrics}

Values of Connectivity metrics have been depicted in Figure 7.6. The lowest COHESION pertains to the class Herbaceous Freshwater Vegetation (77.85), whereas the values of this metric for other classes are more than 90. An increase in COHESION is an indicator of more clumped or aggregated patches of vegetation distributed across the landscape (McGarigal et al., 2012). In addition, in terms of CONNECT values computed, exotic classes seem to be spatially more connected than indigenous ones. In this case, the classes Forest Harvested and Herbaceous Freshwater Vegetation were excluded from the analysis because they consist of only two patches of vegetation. Irrespective of the class Herbaceous Freshwater Vegetation, the classes High Producing Exotic Grassland and Gorse and/or Broom have the highest values in this metric with 4.52 and 2.33, respectively.
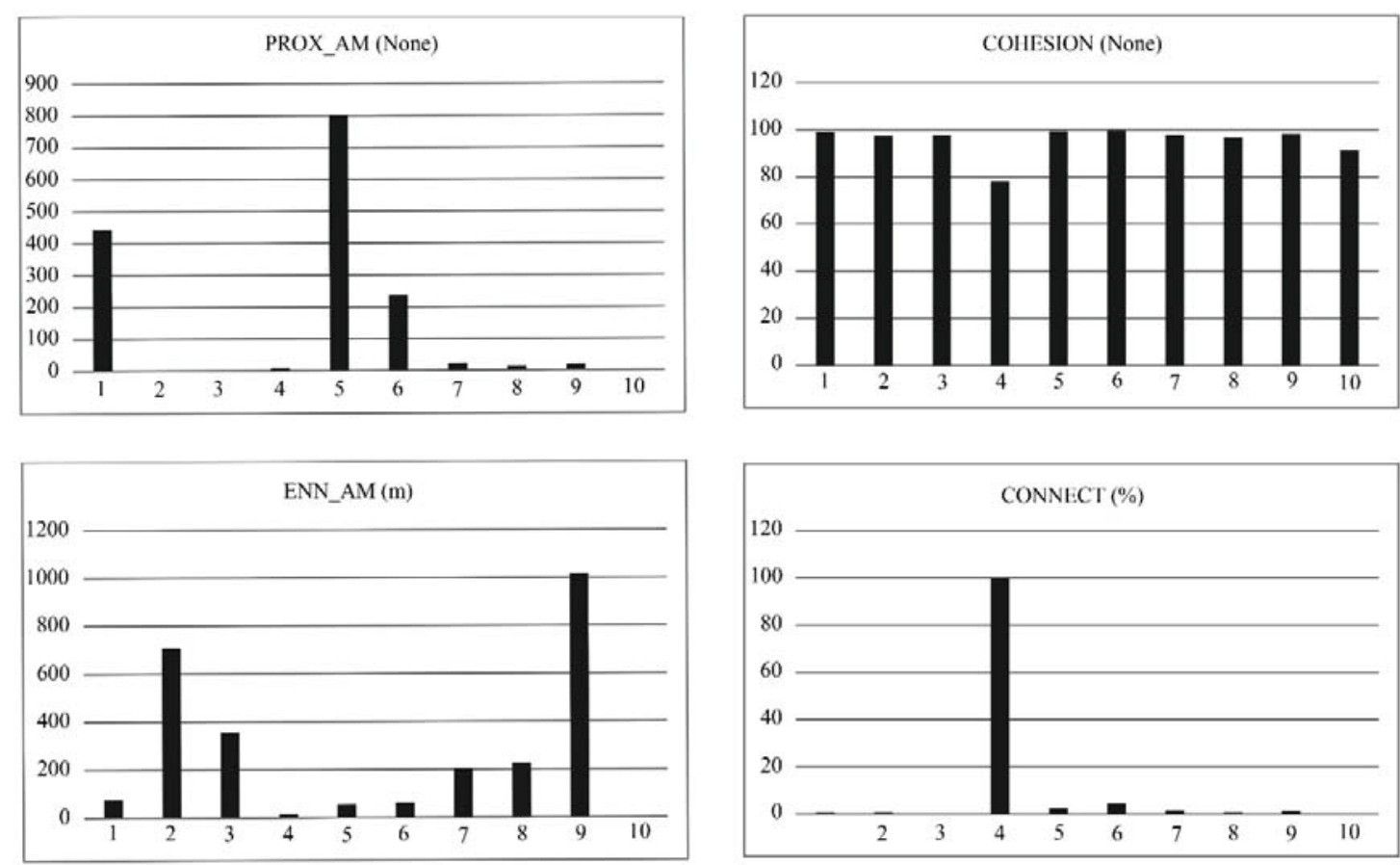

Figure 7.6. Isolation/Proximity and Connectivity Metrics calculated for the 10 land cover classes (cf. Table 7.2). Broadleaved Indigenous Hardwoods (1), Indigenous Forest (2), Matagouri or Grey Scrub (3), Herbaceous Freshwater Vegetation (4), Gorse and/or Broom (5), High Producing Exotic Grassland (6), Exotic Forest (7), Urban Parkland/Open Space (8), Low Producing Grassland (9), Forest Harvested (10). 


\subsection{DISCUSSION}

Results stemming from this research have immediate implications for landscape architecture and land use planning in a Southern Hemisphere urban landscape context. Landscape patterns of a New Zealand urban landscape were quantified and measured to examine if the current composition and configuration of land cover classes are capable of safeguarding indigenous biodiversity against the ill-effects of rising temperatures and intensified winds. Although there seems to be no clear-cut answer to the central question addressed in this chapter, at least eight immediate implications for species persistence under climate change can be enumerated and discussed.

First, allocation of land for future urban development is inevitable ${ }^{54}$. Given that future urban development will occur within the boundaries of the study area (q.v. Chapter 1), developing knowledge of the influence of each land cover class on ecological processes is of critical importance to avoid misallocation of areas of ecological significance to human land use activities. More importantly, human interventions in the spatial patterning of patches of vegetation will have important implications for the selected avifauna, and thereby, urban biodiversity as a whole. Some classes serve as primary or supplementary habitats of urban wildlife, while others play an important role in the process of ecological succession towards indigenous forests. Some may have the least importance for indigenous biodiversity or even threaten it in the long run (q.v. Chapters 4 and 6; Appendix V). The research suggests that future urban development should occur in patches of the least ecological significance or those that are detrimental to the presence of indigenous avifauna.

Second, the spatial pattern of the patches covered by the class Indigenous Forest provides the selected species with unsuitable connectivity and proximity to support seed dispersal mechanisms. However, species movement between patches of vegetation seems to be still possible using stepping stones, particularly in the western part of the Wellington urban landscape, which is home to more than $70 \%$ of the patches of indigenous forests and is a relatively suitable land cover for natural regeneration towards the class Indigenous Forest (Figure 7.7). In Chapters 5 and 6, potentials for biodiversity in this ecological hub were also revealed in terms of landform diversity, indigenousness, and connectivity and proximity (cf. Chapters 5 and 6). Analysis undertaken in this chapter demonstrates that the ecological hub is a suitable zone for safeguarding the selected species in terms of the other components of landscape pattern. Long-term tracking of kererū and tūī shows that in exceptional cases, they are able to fly respectively 11.4 to 101.9 km (Powlesland et al., 2011) and $30 \mathrm{~km}$ (Stevens,

\footnotetext{
${ }^{54}$ According to the Wellington City Council (2015), ca. 21,400 additional dwellings should be provided by the year 2043 in response to increasing urban population growth in the study area (q.v. Chapters 1, 4 and 5).
} 
2006) in search of food sources; however, the seed dispersal ability of these species is very low (ca. $100 \mathrm{~m}$ ) (cf. Wotton and Kelly, 2012). Therefore, small patches of vegetation between primary habitats should be identified to create suitable stepping stones for urban avifauna in public land in order to facilitate species movement and support seed dispersal and natural regeneration mechanisms. As noted in Doody et al. (2010), the participation of landowners is also vital to facilitate species movement and natural regeneration in urban landscapes. Landowners need to be encouraged to plant key indigenous flora in their small properties. At a higher level of participation, they could report the presence of indigenous and exotic mammals in their properties based upon daily observations. This kind of participation and contribution can be discussed under the topic citizen science ${ }^{55}$.
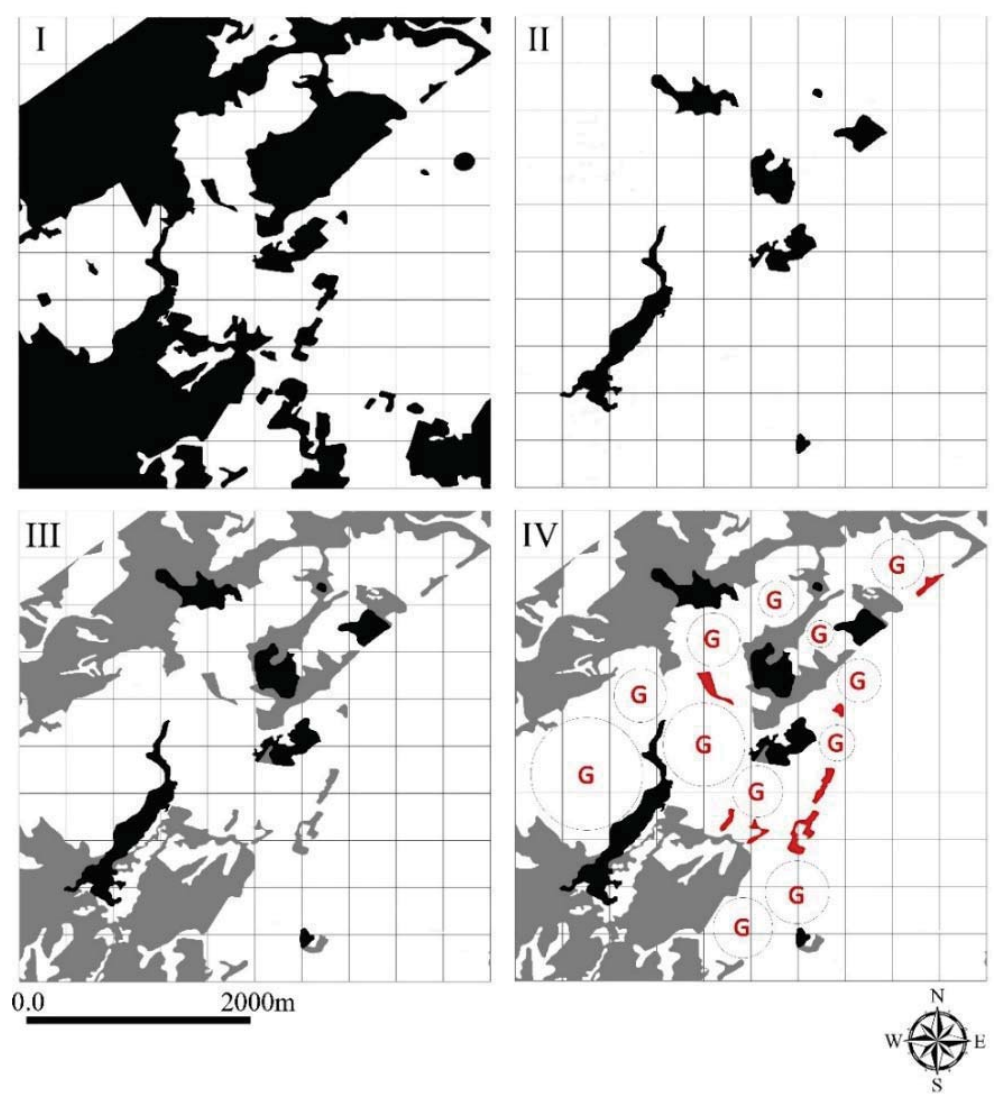

Figure 7.7. The western part of the Wellington urban landscape as a potential hotspot for urban biodiversity (cf. Figures 5.5, 5.6, 6.5, 6.6 and 8.1). I: Green space per capita as a misleading indicator: relatively high proportion of green space per capita comprising different land cover classes (Black) in the urban matrix (White); II: The class Indigenous Forest as the primary habitat of the selected species: composition and configuration of patches of the class Indigenous Forest (Black) in the urban matrix (White); III: Potentials for ecological succession towards the class Indigenous Forest: spatial relation between patches of the class Indigenous Forest (Black) and patches of the classes Broadleaved Indigenous Hardwoods and Gorse and/or Broom (Gray) as nursing patches of vegetation for natural regeneration; IV: Stepping stones and the current spatial gaps: patches of the classes Broadleaved Indigenous Hardwoods and Gorse and/or Broom as potential stepping stones (Red) in relation to spatial gaps (G) between patches of the class Indigenous Forest (Black) and patches of vegetation capable of ecological succession and natural regeneration (Gray).

${ }^{55}$ Data collection based upon citizen science is an important area of research on urban biodiversity. This topic is not in the scope of this research but will be discussed in Chapter 9 as a potential area for future research and inquiry in urban New Zealand. 
Third, although previous studies indicate that very low percentages of the study area consist of the class Indigenous Forest, which serves as primary habitat for indigenous avifauna (Clarkson et al., 2007; cf. Table 4.1; Figure 4.2), with reference to the values of the metrics measuring core area, the present research reveals for the first time that only 36.99 ha of this class serves as interior habitats that can potentially protect species from direct and indirect impacts of climate change. Kererū are sedentary birds with a mean flight distance of 77 m (Wotton, 2007; Wotton and Kelly, 2012), and there is sufficient evidence to indicate that interior habitats are preferable for this species (Barbaro et al., 2012). Hihi are also mostly confined to a pest-free urban wildlife sanctuary in the study area named Zealandia Wildlife Sanctuary comprising at least seven land cover classes including the study area's largest patch of indigenous forest (40.78 ha in total, including 28.94 ha within the administrative boundaries). The hihi population is highly vulnerable to predator pressure (Taylor et al., 2005) and rising temperatures (Chauvenet et al. 2013). Therefore, hihi cannot effectively use edge habitats. Hence, kererū and hihi are examples of New Zealand avifauna that will probably be affected as a result of decreasing the total area of the interior habitats. Similarly, tūi and bellbird use interior habitats. Nevertheless, observations made by the researcher from 2015 to 2017 as well as empirical research shows that they seasonally use edge habitats for foraging (cf. Barbaro et al., 2012). Furthermore, the aforementioned species utilise some exotic plant species for foraging during winters (Gray and van Heezik, 2016). Despite this, the very low quantity of interior habitats covered by the class Indigenous Forest (36.99 ha) is still alarming because predator pressure along edges of habitat patches is higher than core areas due to key factors including food availability and suitable microclimate for the proliferation of some exotic pest species (Paton, 1994; Andrén, 1994; Dijak and Thompson, 2000; Storch et al., 2005).

A field study of indigenous and exotic avifauna in forest edge habitats in New Zealand, for example, evidences a greater presence of exotic species along edges (Barbaro et al., 2012). Innes et al. (2010) rank predation by exotic mammals as the primary cause of decline in populations of kererū, tūī, and bellbirds, among others. Taylor et al. (2005) also show a similar situation for hihi vulnerability (cf. van Heezik et al., 2010; Innes et al., 2015a and b). Thus, although covered by patches of indigenous forest, an increase in edge area may be equivalent to an increase in the odds of predation by exotic fauna given that, as articulated in Chapter 1 , exotic mammals are thought to benefit from rising temperatures (q.v. Table 1.5; Section 1.1.5). As a result, in comparison with habitat area, core area is a more appropriate predictor of high quality forest habitat in urban New Zealand (cf. Temple 1986) (Figure 7.8; cf. Figure 3.3). Aside from predator-prey interactions amongst mammals and avifauna along edges of habitat patches, patches of vegetation with higher edge density are subject to 
higher biological invasion by plants, and therefore, the dominance of invasive exotic flora along the edges of habitat patches is higher (Wiser and Allen, 2006; Hutchison, 2009; also cf. McAlpine et al., 2017). Sheppard et al. (2016) use the term 'invasion hotspot' for New Zealand to emphasise that rising temperatures can lead to the invasion of a wide range of exotic plant species to the country. Light and environmental disturbances caused by extreme weather events provide suitable conditions for alien invasive plant species to grow and expand. Such conditions are common along edges of habitat patches (Daehler, 2003).

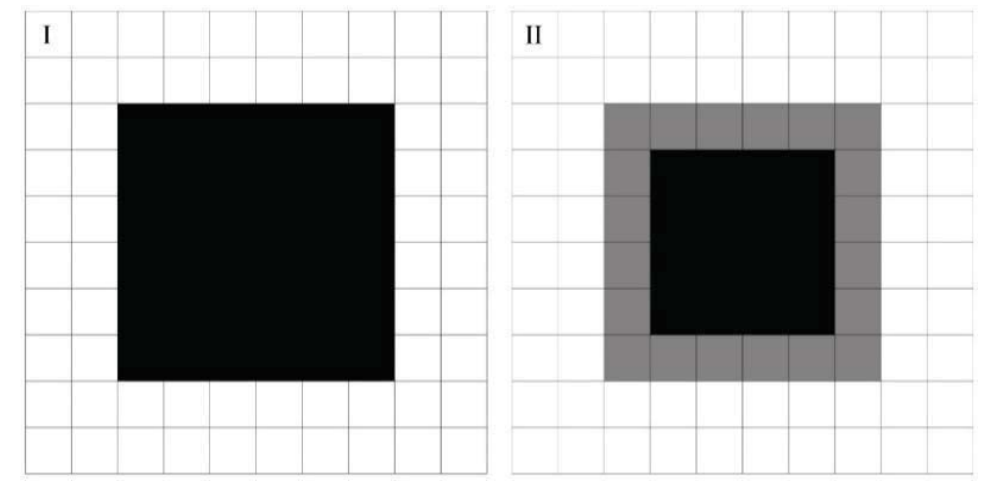

Figure 7.8. Core area as an important indicator for measuring suitability of urban habitats for wildlife species in an era of climate change (cf. Figure 3.3).

Where indigenous species require interior habitats to survive, edge area and interior habitats should be measured separately to provide a real picture of habitat provisioning by urban green spaces. While a given patch of vegetation may seem large enough to safeguard wildlife species from climate change impacts (I), an accurate measurement of edge area can reveal the real area of the habitat (II).

Fourth, the research suggests that the values of CA and PLAND must be interpreted in association with other important metrics measuring interior habitats such as TCA, CPLAND, CORE_AM, and CIA_AM as well as connectivity measurements such as COHESION and CONNECT to build a deeper insight into the real availability of habitat provisioning in urban landscapes. For example, this research reveals that Indigenous Forest, the only primary habitat of indigenous avifauna in urban New Zealand, encompasses a total core area of only $30.34 \%$ of the entire class. This figure shows more degradation of original ecosystems than is typically assumed (cf. Clarkson et al., 2007; cf. Table 4.1). Consequently, this could lead to greater indigenous biodiversity loss as climate change impacts increase. Moreover, this class has the least suitable conditions in terms of proximity compared to other classes. This in turn, may influence species dynamics and gene exchange between patches of the class Indigenous Forest and wildlife within them. Collectively, patches covered by this class are typically isolated, and proximity between them is very low. In contrast, lower values of ENN_AM and higher values of PROX_AM for exotic classes, as computed here, can facilitate the spread of weeds and pests across the landscape. 
Fifth, relatively low values of Indigenous Forest in core metrics (i.e. TCA, CPLAND, CORE_AM, and CAI_AM) and also connectivity metrics (i.e. COHESION and CONNECT) represent dysfunctionality of the class in terms of providing suitable interior habitats as well as spatial connectivity for seed dispersal and therefore ongoing ecosystem health and natural regeneration over time. With reference to the value of GYRATE_AM, however, the extensiveness of the patches covered by this class is proportionally high (Figure 7.9). This heralds opportunities for biodiversity conservation at the patch level (vs. landscape level). In the absence of enough connectivity and proximity between patches of indigenous forest at the present time, the establishment of pest-free urban wildlife sanctuaries with the high proportion of the class Indigenous Forest seems to be essential (q.v. Chapter 8). To enable this, the radius of gyration is suggested as a strong indicator of habitat suitability for the site selection of urban wildlife sanctuaries in the coming decades.

Sixth, the class Exotic Forest is very likely to affect adjacent patches of vegetation because the higher values of ED and NP computed for this class can be regarded as a threat to the selected avifauna. Despite relatively low values of CA (842.7 ha) and PLAND (6.23\%), high values of ED and NP in this class can lead to higher interactions with the surroundings and this in turn will facilitate the spread of weeds and pests already colonised in patches of exotic forest (i.e. exotic seeds and introduced mammals that predate upon indigenous avifauna). This is because elongated patches of vegetation are thought to have more interactions with their surroundings (Dramstad, et al., 1996). This situation may become more problematic given that spatial distance between patches covered by this class is not high.

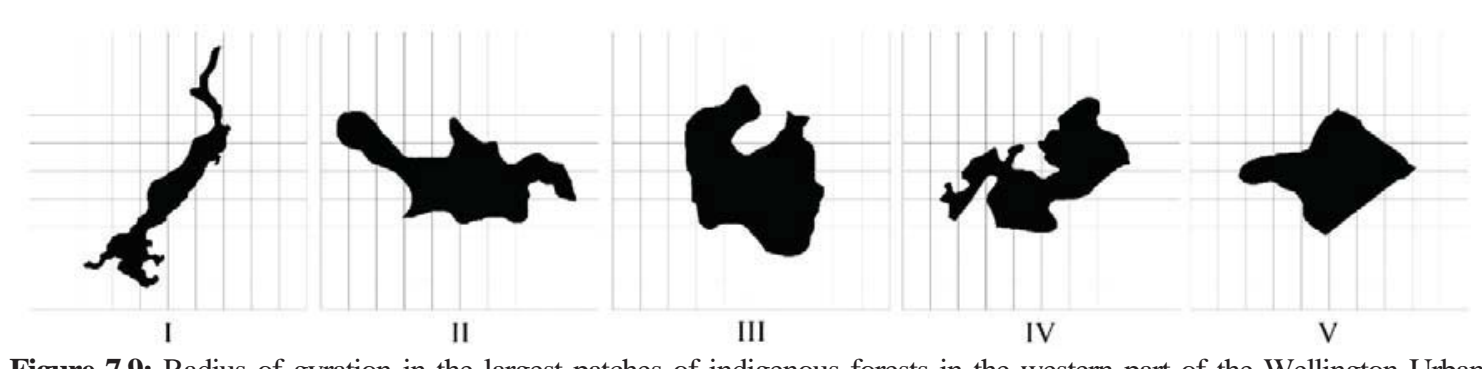

Figure 7.9: Radius of gyration in the largest patches of indigenous forests in the western part of the Wellington Urban landscape, namely Zealandia Wildlife Sanctuary (I); Otari-Wilton’s Bush (II); Town Belt (III and V); Wellington Botanic Garden (IV) (not to scale). The depicted patches of indigenous forest collectively comprise 83.3 ha (68.44\%) of the class Indigenous Forest in the study area (cf. Figures 6.5 and 8.3).

Seventh, 731.99 ha of the class Gorse and/or Broom consist of interior habitats. Although the availability of interior habitats is not an important factor for this class per se, patches of gorse and/or broom with sufficient core areas can be regarded as targeted patches for landscape restoration more 
specifically because the patches of vegetation covered by this class have a high degree of proximity (PROX_AM = 801.84) and a low value of spatial distance (ENN_AM = 54.87). Thus, in line with taking geo-environmental factors such as topography, distance from seed source, and humidity into account, as suggested in the literature (Davis and Meurk, 2001; q.v. Chapter 6), the potential availability of interior habitats can also be important as a criterion to identify candidate patches of gorse and/or broom to initiate landscape restoration practices. Patches of gorse and/or broom with enough space for providing interior habitats should take precedence for landscape restoration practices if other environmental requirements are simultaneously met.

Eighth, although green space development is believed to be a proper response to challenges such as urban heat island effects (Myint et al., 2015; Rhee et al., 2014), increasing the percentage of green spaces is not necessarily an appropriate response to biodiversity conservation in the face of climate change in the Southern Hemisphere in general and in New Zealand in particular. In terms of green space per capita, Wellington is one of the world's greenest cities (400 $\mathrm{m}^{2}$ per capita) (q.v. Chapter 4). Despite this, Wellington's relatively large quantity of urban green space cannot efficiently support the avifauna under study because the study area's indigenous classes do not provide the selected species with enough interior habitats (Figure 7.10). At the same time, exotic classes are spatially widespread and dispersed across the landscape, affecting healthy functioning of ecosystems supported by indigenous flora and fauna. Furthermore, the current landscape pattern is not capable of facilitating species movement between patches of indigenous vegetation. This situation for other cities would be even more challenging because the Wellington green space per capita is considerably higher than international standards (cf. Singh et al., 2010). The research highlights that considering the quantity of urban green space as an indicator for biodiversity conservation can be, to a large extent, misleading. Green space per capita is not necessarily a proper indicator of potential urban biodiversity resilience in the face of climate change. Rather, it is the composition and configuration of patches of vegetation that should be taken into particular account.

Landscape pattern analysis would be most accurately interpretable when including data relating to the spatial ecologies of a specific group of keystone species in the computation. It is impossible in practice to study all species in an urban landscape (q.v. Section 1.4.3). This research suggests a methodology for using data related to a limited number of keystone species for interpreting the GIS and FRAGSTATS outputs. The results can be generalised, with a high degree of certainty, because keystone species often represent the same ecological traits and niches of a wide range of other avifauna endemic to the region (cf. Meurk and Hall, 2006; Meurk et al., 2016). 


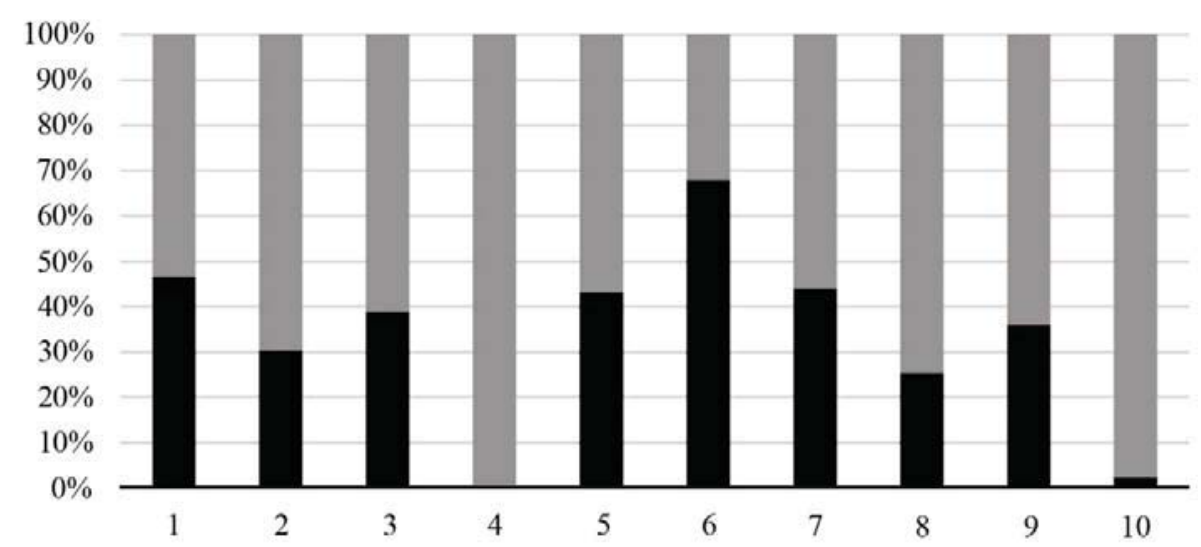

Figure 7.10. Core habitats (black) vs. edge areas (grey) measured for 10 land cover classes in the study area (cf. Table 7.2). Broadleaved Indigenous Hardwoods (1), Indigenous Forest (2), Matagouri or Grey Scrub (3), Herbaceous Freshwater Vegetation (4), Gorse and/or Broom (5), High Producing Exotic Grassland (6), Exotic Forest (7), Urban Parkland/Open Space (8), Low Producing Grassland (9), Forest Harvested (10).

Although the aforementioned implications can contribute to a higher level of informed interventions in landscape patterns for biodiversity conservation in urban landscapes, this study should be regarded as a starting point. More investigation is needed based upon field surveys in the future. At this stage, while empirical data on the behaviours of species in relation to landscape patterns is still scattered or, in part, lacking, the research employed an alternative methodology to contribute to the knowledge of informed landscape architecture and land use planning practices in urban landscapes, and at the same time, the research created a platform for further research on this topic in urban New Zealand or internationally. Landscape pattern analysis would be more accurately interpretable when including data relating to the spatial ecologies of a specific group of species into the computation. It is impossible in practice to study all species in an urban landscape.

In order to provide more empirical evidence on species' behaviour in space and time in relation to landscape patterns and climate change impacts, an informed use of GPS telemetry, GISbased mapping and FRAGSTATS-aided computation is highly recommended. This may be one of the most effective research approaches for filling the gaps relating to a lack of data on the relationships between landscape patterns and ecological processes based upon site-specific ecological traits and niches. Empirical research should be undertaken to test the validity level of information and consequent results presented in this chapter in New Zealand or internationally.

\subsection{CONCLUSION}

This research provides, for the first time, an in-depth understanding of the study area's current landscape pattern composition and configuration in relation to the spatio-temporal behaviours of four 
keystone species using GIS, FRAGSTATS, and a core set of landscape metrics. Results contribute to the evidence that increasing the percentage of green spaces is not necessarily an appropriate response to biodiversity loss in the face of some impacts of climate change. Instead, it is the landscape composition and configuration of habitat patches that will play a more important role in safeguarding wildlife against the ill-effects of the changing climate. In addition, the research reveals that changes in patch size, connectivity and proximity, edge density, and shape complexity influence avifauna in different ways depending on the biological nature of land cover classes. This, accordingly, led to eight immediate implications for landscape architecture and land-use planning practices in a changing climate. These implications can give rise to a higher level of informed decision-making on a wide range of practices for biodiversity conservation under uncertainties associated with climate change, including site selection of potential wildlife habitats, strategic stepping stones, areas capable of natural regeneration, and suitable places for future housing and infrastructure development as well as the identification of areas susceptible to biological invasion by exotic flora and fauna. This, in turn, will provide useful information about how to configure/reconfigure and compose/recompose current landscape patterns in a way that proactively helps safeguard urban biodiversity in an era of climate change. This demonstrates one crucial way of preserving or increasing ecosystem services as a means of increasing human resilience to climate change in urban landscapes. In line with these class-level landscape strategies, a number of pest-free urban wildlife sanctuaries are concurrently required to support other fauna that may be more vulnerable to direct and indirect impacts of climate change and need pest-free habitat patches to survive (q.v. Chapters 1 and 3). This issue will be addressed and discussed in Chapter 8. 


\section{CHAPTER 8}

\section{Study III: The potential for establishing urban wildlife sanctuaries}

\subsection{RESEARCH AIMS}

In Chapter 7, the research indicated that in line with spatial management of land cover patterns at the class and landscape levels, a number of pest-free urban wildlife sanctuaries is also needed in the Wellington urban landscape in order to ensure the survival of indigenous fauna against increased predator pressure resulting from rising temperatures. This argument was strongly supported by New Zealand subject-matter experts in Study II (q.v. Chapter 3). In this chapter, the main aims are to:

(1) Examine the potential for the establishment of pest-free urban wildlife sanctuaries in the study area - the Wellington urban landscape (q.v. Figure 1.6);

(2) Examine how the eight most important components of landscape pattern can be utilised as spatial criteria to identify the most suitable sites for such a purpose.

\subsection{INTRODUCTION}

As articulated in Chapter 1, exotic mammals imported to New Zealand by early European immigrants are estimated to benefit from rising temperatures and this, consequently, increases the risk of predator pressure on indigenous fauna in New Zealand urban landscapes (q.v. Chapter 1). Due to the fragile nature of New Zealand indigenous fauna, pest control is essential to support long-term indigenous biodiversity in urban landscapes. This concern was also raised in Study II (q.v. Chapter 3). There is a wide range of evidence to suggest that the successful conservation of New Zealand indigenous fauna depends profoundly on pest control (Brown, 1997; Gillies and Clout, 2003; van Heezik et al., 2008 b; Innes et al., 2012; van Heezik et al., 2010; Aguilar et al., 2015; Goldson et al., 2015; Russell et al., 2015). Research on little-spotted kiwi (McLennan et al., 1996; Robertson and Colbourne, 2004), tuatara (Jarvie et al., 2016), avifauna in general (Duncan and Blackburn, 2004; Blackburn et al., 2005; Innes et al., 2015 a), and reptiles (Reardon et al., 2012) indicates that the 
presence of introduced exotic mammals is a serious threat to the survival of the aforementioned species.

Zealandia, the first pest-free urban wildlife sanctuary in New Zealand (Beatley, 2016), is a successful example of the employment of specific strategies to respond to urban biodiversity loss. This urban wildlife sanctuary, similar to other New Zealand examples (e.g. Travis Wetland Nature Heritage Park in Christchurch), has a very special structure, a specific fence construction, a strategic visiting regime, and a management system that provide a pest-free, and accordingly disease-free, habitat for not only a wide range of vulnerable fauna, but also indigenous flora in an urban context. Zealandia was established in 1999 (Beatley, 2016) to support indigenous biodiversity in the Wellington urban landscape, but not necessarily as a refugium for indigenous fauna in the face of climate change impacts.

Although connectivity between patches of vegetation is essential to facilitate species movement and contribute to natural regeneration through seed dispersal and pollination mechanisms in urban New Zealand (q.v. Chapters 6 and 7), pest-proof fencing is concurrently recommended by New Zealand researchers for maintaining biodiversity in urban landscapes when biodiversity is addressed at the patch level (Burns et al., 2012; Innes et al., 2012; Empson and Fastier, 2013; Innes et al., 2015 b; Norton et al., 2016). This fact was also discussed in Study II (q.v. Chapter 3). Although some flying New Zealand avifauna can still avail themselves of connectivity, highly vulnerable species such as little-spotted kiwi, tuatara, takahē, hihi, and kākā are necessarily required to be protected from predation pressure by pest-proof fencing because they are not as mobile as New Zealand avifauna and/or their populations are not as large as other species such as New Zealand lizards and skinks. Therefore, site selection for the identification of the most suitable urban wildlife sanctuaries is vital to support these vulnerable species in an era of climate change. Pest-free urban wildlife sanctuaries can simultaneously contribute to the presence and abundance of a wide range of other indigenous species endemic to New Zealand.

\subsection{MATERIALS AND METHODS}

\subsubsection{Multi-criteria analysis}

A six-step process was designed, based upon Hill et al., 2005; Svoray et al., 2005; Wang and Hofe, 2008; Zucca et al., 2008; Huang et al., 2011; Fontana et al., 2013; Fernandez and Morales, 2015; Langemeyer et al., 2016, to perform a multi-criteria analysis of the most suitable patches of 
vegetation spatially capable of serving as urban wildlife sanctuaries in an era of climate change. This is a cost-effective and relatively efficient method to identify and rank the candidate sites depending on expert knowledge, spatial data, and GIS analysis.

\subsubsection{Step 1: Identifying candidate sites}

Approximately $60 \%$ of the Wellington urban landscape is covered by different types of green spaces ranging from indigenous, mixed, and exotic land cover classes. The rest is covered by built-up areas or non-vegetative patches (q.v. Table 4.1). This extent of green space comprises hundreds of patches of vegetation (q.v. Table 7.2). As green spaces differ in terms of habitat quality and ecological characteristics, it is necessary to focus on a limited number of candidate sites that are socially and/or ecologically apt for this purpose. First, three primary criteria were taken into consideration to select the most relevant candidate sites among a larger pool of patches of vegetation (Table 8.1). Doing this also reduced the number of candidate sites to an acceptable level. This is a common method in multicriteria suitability analysis (Wang and Hofe, 2008; Deming and Swaffield, 2011). These primary criteria were devised to make sure that the output of this research is transferrable to the real world. For example, private land was excluded from the process of site selection using Wellington City Council Open Data (2015). This is because it seems impractical to establish urban wildlife sanctuaries on private properties even though spatial requirements are met in some of these locations. The rationale for each criterion has been described in Table 8.1. Applying the primary criteria, four candidate sites were identified (Table 8.2). The selected candidate sites meet the primary criteria, are located in the western part of the Wellington urban landscape (i.e. ecological hub), and encompass more than 68\% of the class Indigenous Forest in the Wellington urban landscape (Figure 8.1). From a biological point of view, the class Indigenous Forest is the most important land cover class in New Zealand, representative of New Zealand forests before the arrival of humans, and it is of the highest habitat quality compared to other classes (Chapters 3 and 4; Appendix V). Excluding Zealandia, the other three candidate sites are not currently fenced to support vulnerable indigenous fauna. LCDB (q.v. Section 4.3) and NZDEM (q.v. Sections 5.3.2 and 6.3) were used to quantify the selected candidate sites in the Arc Map v.10.4.1 environment (Landcare Research, 2010; 2015). Specifically, FRAGSTATS v.4.2. (McGarigal et al., 2012) was also used to quantify and measure shape complexity based upon the metric Shape Index - SHAPE (Botequilha Leitão et al., 2006). As a size-

independent algorithm, Shape Index is the most relevant landscape metric to quantify shape complexity (McGarigal et al., 2012; q.v. Appendix VII). 
Table 8.1

Primary criteria for the identification of candidate sites.

\begin{tabular}{ll}
\hline Criteria & Rationale \\
\hline Indigenousness & $\begin{array}{l}\text { Areas covered by the class Indigenous Forest play an irreplaceable role in the provision of } \\
\text { habitat and food for indigenous fauna in New Zealand (q.v. Chapter 3; Appendix V). }\end{array}$ \\
Patch size & $\begin{array}{l}\text { Larger patches of vegetation can support greater biodiversity over time (q.v. Chapter 2). } \\
\text { Based upon the current literature and discussions made in the course of Study II (q.v. } \\
\text { Chapter 3), a threshold size of } 25 \text { ha was applied to identify the largest patches of } \\
\text { vegetation. }\end{array}$ \\
Land ownership & $\begin{array}{l}\text { The establishment of urban wildlife sanctuaries would be more feasible in public lands or } \\
\text { areas that are currently managed for biodiversity conservation. }\end{array}$ \\
\hline
\end{tabular}

Table 8.2

Total area and percentage of the candidate sites covered by the class Indigenous Forest.

\begin{tabular}{lrrr}
\hline Candidate site & Area (ha) & Indigenous Forest (ha) & \% in the study area \\
\hline Town Belt & 98.8 & 22.27 & $18.32 \%$ \\
Botanic Garden & 25.6 & 15.39 & $12.56 \%$ \\
Zealandia & 247.6 & 28.94 & $23.74 \%$ \\
Otari-Wilton's Bush & 81.2 & 16.71 & $13.72 \%$ \\
Total & 453.2 & 83.31 & $68.34 \%$ \\
\hline
\end{tabular}

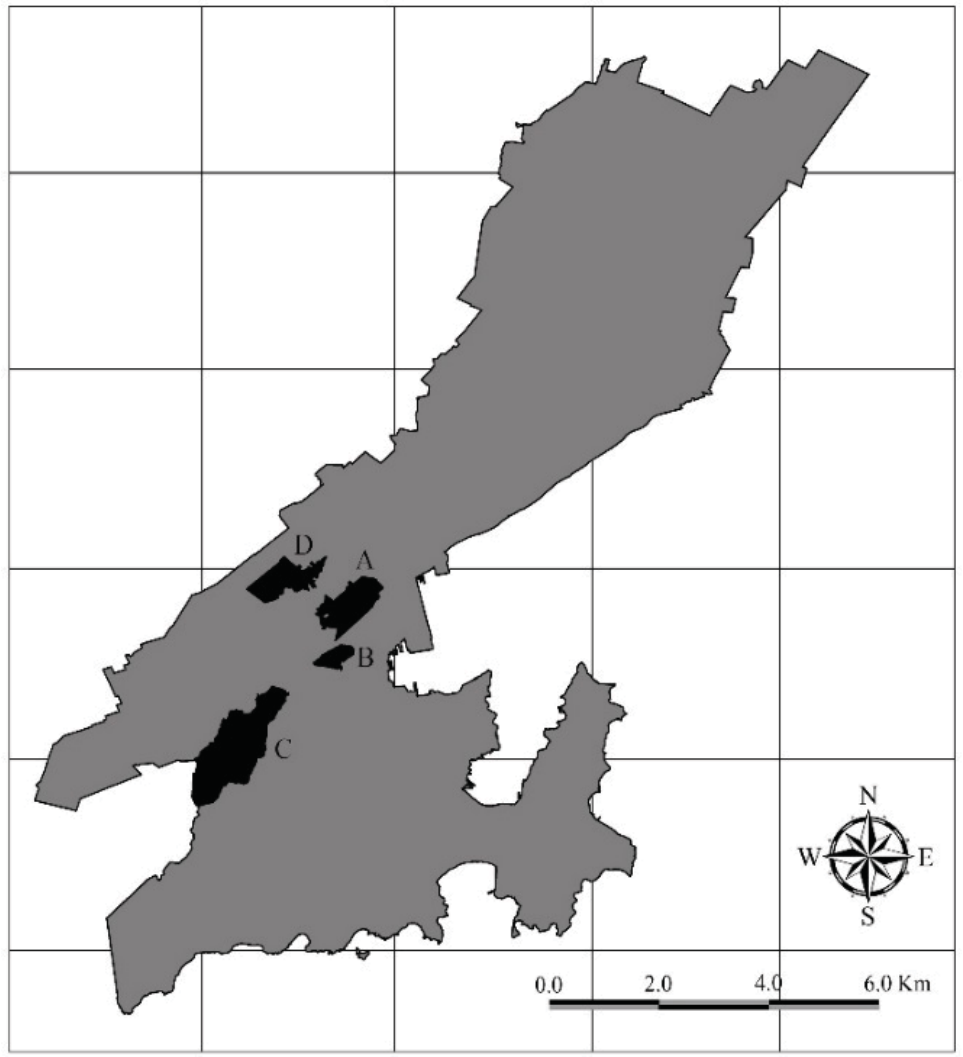

Figure 8.1. Candidate sites: Town Belt (A), Botanic Garden (B), Zealandia (C), and Otari-Wilton’s Bush (D) (cf. Figures 5.5, 5.6, 6.5, 6.6 and 7.7). 


\subsubsection{Step 2: Determining factors}

Based upon Studies I and II (q.v. Chapters 2 and 3), eight components of landscape pattern affecting the presence, abundance, and richness of fauna in urban landscapes were regarded as main factors for analysis (Table 8.3; cf. Table 2.5; Section 3.3.1.1).

While south-facing slopes covered by the class Indigenous Forest are considered as suitable places for wildlife in the face of rising temperatures (q.v. Chapter 5), it was argued that landform diversity, per se, is a determinant geophysical factor in providing diverse micro-climatic conditions for wildlife in the event of various climate change impacts (q.v. Section 5.2). For example, while southfacing slopes may be safer locations in warmer than average temperatures, these areas are not necessarily safe for wildlife in the face of gales, storms, flooding, wildfire, and the spread of pests (i.e. predators) and weeds. In this analysis, landform diversity was used as a stronger criterion to test whether the candidate sites provide diverse habitats for wildlife in the face of diverse climatic conditions.

\subsubsection{Step 3: Dedicating weights to factors}

To dedicate an appropriate weight to each factor, expert knowledge was utilised. Thus, weights proposed in Study I (q.v. Chapter 2) and confirmed in Study II (q.v. Chapter 3) were considered for this research (q.v. Table 8.3). 
Table 8.3

Weights dedicated to the eight most important components of landscape pattern (q.v. Tables 2.1 and 2.5; Section 3.3.1.1).

\begin{tabular}{llr}
\hline Factors & Definition & Weight \\
\hline Indigenousness & $\begin{array}{l}\text { The state of being indigenous/native in terms of land } \\
\text { cover classes present in an urban context. }\end{array}$ & 0.1377 \\
Land cover heterogeneity & $\begin{array}{l}\text { The diversity of different land cover classes in patch or } \\
\text { landscape levels. }\end{array}$ & 0.1449 \\
Land surface perviousness & $\begin{array}{l}\text { The ability of a particular land cover or landscape to } \\
\text { absorb run-off caused by rainfall or sequester and store } \\
\text { carbon dioxide in soil or vegetation. }\end{array}$ & 0.1188 \\
Patch size & $\begin{array}{l}\text { Total area of a particular land cover on a patch or } \\
\text { landscape level. }\end{array}$ & 0.1639 \\
Connectivity and proximity & $\begin{array}{l}\text { The spatial distance between patches of a particular land } \\
\text { cover class. }\end{array}$ & 0.1449 \\
Edge density & $\begin{array}{l}\text { Perimeter of a patch of particular land cover class } \\
\text { exposed to other land cover class. }\end{array}$ \\
Landform diversity & The diversity of elevations, slopes, and aspects. \\
\hline Shape complexity & $\begin{array}{l}\text { The degree to which a wildlife habitat is dissimilar to } \\
\text { circle-shaped pattern. }\end{array}$ \\
\hline
\end{tabular}

\subsubsection{Step 4: Defining attribute values and associated scores for each factor}

Looking at the current literature as well as discussions made in the course of Studies I and II (q.v. Chapters 2 and 3), a suitability spectrum was designed in terms of factors identified in Step 2 above in order to provide a framework for defining attribute values (Figure 8.2). Possible attribute values for each factor were defined based upon the current conditions using field surveys followed by a GIS analysis of the current composition and configuration of the four candidate sites (i.e. Town Belt, Botanic Garden, Zealandia, and Otari-Wilton's Bush). Field surveys were conducted to observe various aspects of the candidate sites. Although informal, the aim was to visit the candidate sites in order to become more familiar with their 'on the ground' nature and the ecological characteristics of the spaces. Each candidate site was visited at least twice and an informal survey was made on foot. As much as possible of the boundary of each candidate site was covered on foot, as were easily assessable existing paths in the interiors. Photos of each site were taken from different angles. This enabled the researcher to build and record a deeper understanding of the status and nature of each candidate site as well as experiencing and noting its general socio-ecological atmosphere ${ }^{56}$ (cf.

\footnotetext{
${ }^{56}$ Contrary to the casual observations mentioned in Chapter 4 (q.v. Footnote 47), the aim of observations in this stage was not to compare the accuracy of spatial data and the extent of land cover classes in the real environment. It was simply to experience the candidate sites in person.
} 
Footnote 47). A set of scoring criteria was also defined to dedicate an individual score to each attribute value (Table 8.4).

Table 8.4

Scoring criteria moderated based upon local conditions applied to dedicate scores to each factor's attribute.

\begin{tabular}{|c|c|}
\hline Factors & Scoring criteria applied in the scoring system \\
\hline Indigenousness & $\begin{array}{l}\text { If } 100 \% \text { of the site is covered by the class Indigenous Forest, a score of } 5 \text { will be } \\
\text { dedicated as the highest possible score. Other attributes will be relatively scored } \\
\text { based upon this rule. }\end{array}$ \\
\hline Land cover heterogeneity & $\begin{array}{l}\text { If the number of land cover classes is } 9 \text {, a score of } 5 \text { will be dedicated as the } \\
\text { highest possible score. Other attributes will be relatively scored based upon this } \\
\text { rule. }\end{array}$ \\
\hline Land surface perviousness & $\begin{array}{l}\text { If } 100 \% \text { of the site is covered by patches of vegetation, a score of } 5 \text { will be } \\
\text { dedicated as the highest possible score. Other attributes will be relatively scored } \\
\text { based upon this rule. }\end{array}$ \\
\hline Patch size & $\begin{array}{l}\text { A score of } 5 \text { will be dedicated as the highest possible score to the largest candidate } \\
\text { site. Other attributes will be relatively scored based upon this criterion. }\end{array}$ \\
\hline Connectivity and proximity & $\begin{array}{l}\text { If the spatial distance between the candidate site and the nearest patch of } \\
\text { indigenous forest is less than } 100 \mathrm{~m} \text {, a score of } 5 \text { will be dedicated as the highest } \\
\text { possible score. Other attributes will be relatively scored based upon this rule. }\end{array}$ \\
\hline Edge density & $\begin{array}{l}\text { A score of } 5 \text { will be dedicated as the highest possible score to a candidate site with } \\
\text { the lowest value of edge density. Other attributes will be relatively scored based } \\
\text { upon this rule. }\end{array}$ \\
\hline Landform diversity & $\begin{array}{l}\text { A score of } 5 \text { will be dedicated as the highest possible score to a candidate site with } \\
\text { the highest rate of topographical diversity. Other attributes will be relatively scored } \\
\text { based upon this rule. }\end{array}$ \\
\hline Shape complexity & $\begin{array}{l}\text { Due to the lack of theoretically compact patches of vegetation (i.e. circle-shaped), } \\
\text { a score of } 3 \text { will be dedicated as the relatively medium possible score to the most } \\
\text { compact candidate site. Other attributes will be relatively scored based upon this } \\
\text { criterion. }\end{array}$ \\
\hline
\end{tabular}
criterion.

\subsubsection{Step 5: Calculating weighted scores for each factor}

The weighted score of each factor for each candidate site was calculated based upon the existing attribute of the candidate site using the following formula:

$$
W S_{i}=W_{i} \times S_{i}
$$

Where $W S_{i}$ is the weighted score of factor $i, W_{i}\left(0<W_{i}<1\right.$ and $\left.\sum W_{i}=1\right)$ is the weight assigned to the factor $i$, and $S_{i}$ is the score dedicated to the attribute value of factor $i$. 


\subsubsection{Step 6: Calculating composite scores for each candidate site}

Ultimately, the composite score of each candidate site was calculated using the following formula:

$$
C S_{i}=\sum\left(W S_{i}\right)
$$

Where $C S_{i}$ is the composite score of factor $i$, and $W S_{i}$ is the weighted score of factor $i$.

This six-step process was fulfilled to rank and prioritise the identified candidate sites in terms of spatial suitability for safeguarding indigenous fauna in the face of climate change impacts including increased predator pressures and the spread of diseases among vulnerable indigenous fauna.

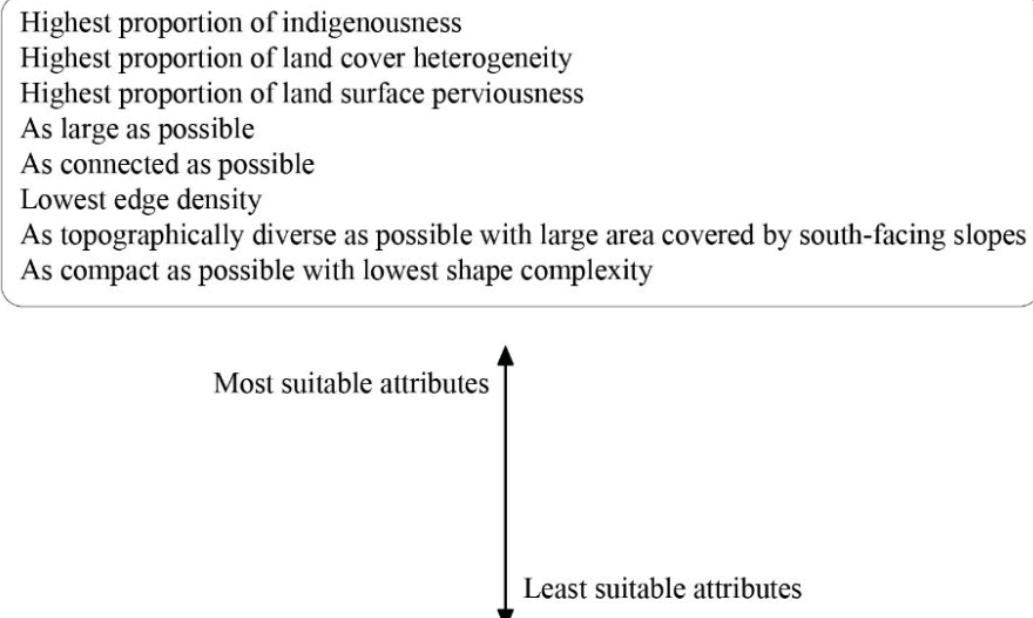

Figure 8.2. Suitability spectrum used to define attribute values (cf. Tables 8.4 and 2.1).

\subsection{RESULTS}

Detailed information about the extent of land cover classes in the candidate sites has been provided in Tables 8.5 and 8.6. Zealandia, Town Belt, Otari-Wilton’s Bush, and Botanic Garden respectively cover 247.6ha, 98.8ha, 81.2ha, and 25.6 ha of the study area (total area $=13511$ ha) 
including a range of land cover classes of ecological significance. The largest patches of indigenous forest were identified in Zealandia (28.94 ha), Town Belt (22.27 ha), Otari-Wilton’s Bush (16.71 ha), and Botanic Garden (15.39 ha); however, in terms of the ratio of the candidate site's area to indigenous forest's area, Botanic Garden occupies the first place because more than $60 \%$ of its total area has been covered by the class Indigenous Forest.

Table 8.5

Land cover classes consisting of plant life in the candidate sites (cf. Figure 8.3; Table 8.6).

\begin{tabular}{lcll}
\hline Candidate sites & Area (ha) & Indigenous classes & Exotic classes \\
\hline Town Belt & 98.8 & $\begin{array}{l}\text { Broadleaved Indigenous Hardwoods } \\
\text { Indigenous Forest }\end{array}$ & $\begin{array}{l}\text { Urban Parkland/Open Space } \\
\text { Exotic Forest } \\
\text { Gorse and/or Broom }\end{array}$ \\
Botanic Garden & 25.6 & $\begin{array}{l}\text { Broadleaved Indigenous Hardwoods } \\
\text { Indigenous Forest }\end{array}$ & Urban Parkland/Open Space \\
Zealandia & 247.6 & $\begin{array}{l}\text { Broadleaved Indigenous Hardwoods } \\
\text { Indigenous Forest } \\
\text { Herbaceous Freshwater Vegetation }\end{array}$ & $\begin{array}{l}\text { Exotic Forest } \\
\text { Gorse and/or Broom }\end{array}$ \\
& 81.2 & $\begin{array}{l}\text { Broadleaved Indigenous Hardwoods } \\
\text { Indigenous Forest }\end{array}$ & $\begin{array}{l}\text { Exotic Forest } \\
\text { Gorse and/or Broom } \\
\text { Otari-Wilton's Bush }\end{array}$ \\
& & & Low Producing Grassland \\
\hline
\end{tabular}

Spatial attributes measured for the candidate sites in terms of the eight factors affecting the behaviours of fauna in space and time were quantified and tabulated (Tables 8.7 and 8.8) using LCDB (Landcare research, 2015), New Zealand Digital Elevation Model North Island 25 m (Landcare Research, 2010), and FRAGSTATS v.4.2 (McGarigal et al., 2012). 


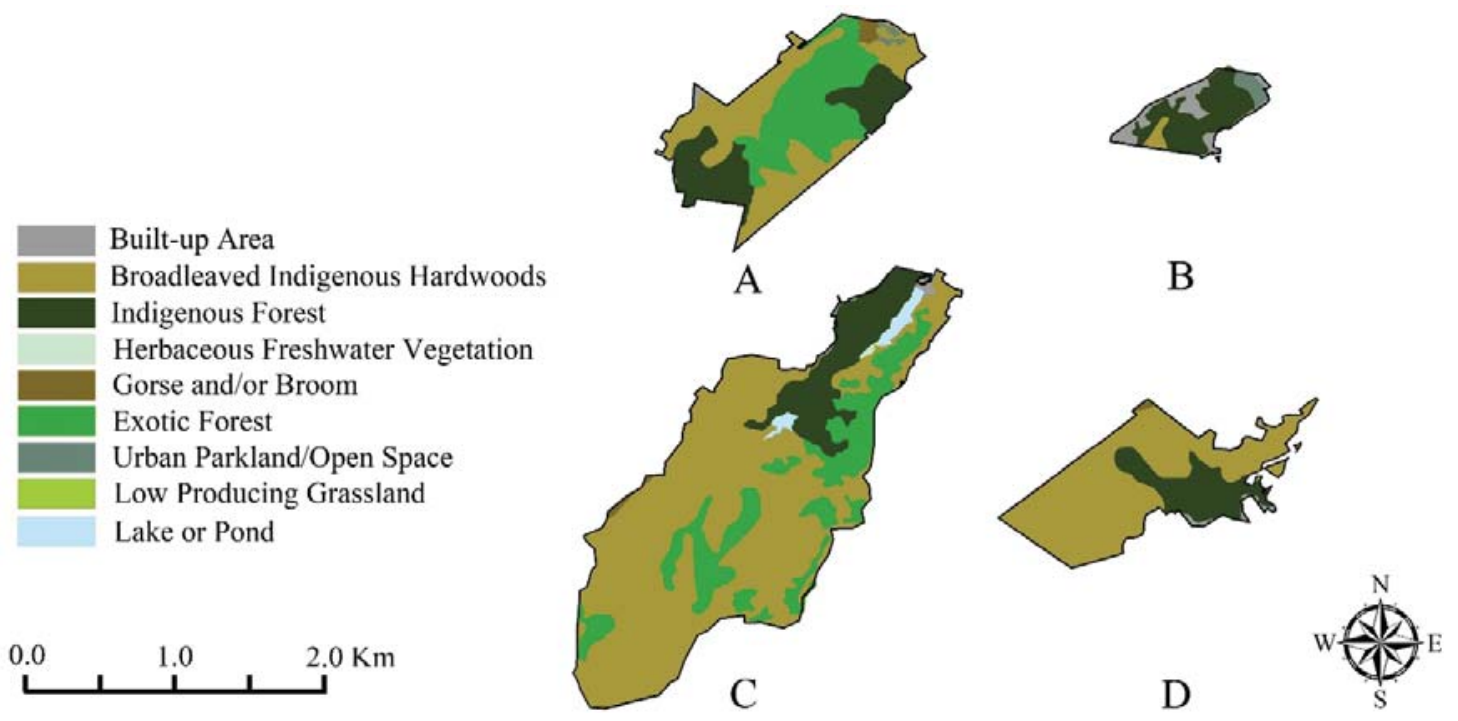

Figure 8.3. Land cover classes in the candidate sites: Town Belt (A), Botanic Garden (B), Zealandia (C), and Otari-Wilton’s Bush (D) (cf. Tables 8.5 and 8.6).

Zealandia is the most heterogeneous candidate site in terms of the number of land cover classes, including the classes Built-up Areas and Lake or Pond ( $\mathrm{n}=7$ ) (q.v. Figure 8.3). In addition, the least pervious candidate site is Botanic Garden (land surface perviousness $=91.47 \%$ ) compared to Zealandia (99.44\%), Town Belt (98.62\%), and Otari-Wilton’s Bush (98.09\%). Botanic Garden and Town Belt are the most connected candidate sites $(255 \mathrm{~m})$, while the spatial distance between Zealandia and the nearest patches of indigenous forest is $698 \mathrm{~m}$. The lowest edge density belongs to Botanic Garden (2671m) whereas the values of edge density for other candidate sites are relatively high. Due to the lack of detailed spatial data, landform diversity cannot be easily measured on a fine scale in urban New Zealand. Despite this, a qualitative measurement of this factor followed by a GISbased analysis of the candidate sites using NZDEM (q.v. Chapters 5 and 6) shows that the highest landform diversity is available in Zealandia. Finally, in terms of shape complexity, a FRAGSTATSaided analysis of the candidate sites based upon the landscape metric Shape Index (McGarigal et al., 2012; q.v. Appendix VII) reveals that the most compact candidate sites are Botanic Garden (26.56), Otari-Wilton’s Bush (33.23), Town Belt (42.17), and Zealandia (44.59), respectively. Ultimately, normalised and weighted composite scores of the candidate sites reveal that Zealandia (2.918) and Botanic Garden (2.769) are potentially the most important candidate sites in terms of spatial capability to safeguard the selected keystone species against the local impacts of climate change. Otari-Wilton's Bush (2.607) and Town Belt (2.325) stand in third and fourth places, respectively (Table 8.9). 
$\stackrel{\infty}{\varrho}$

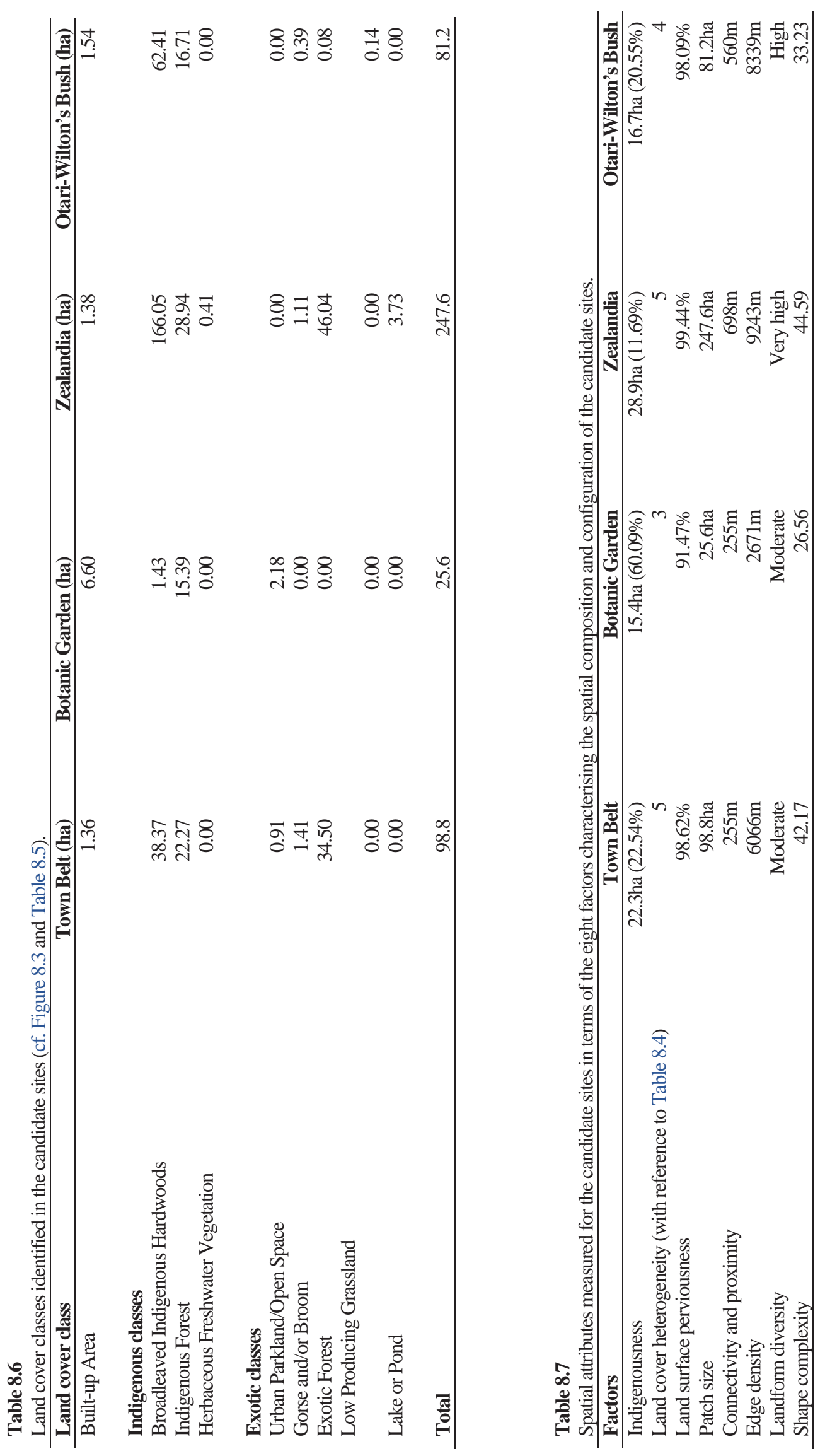



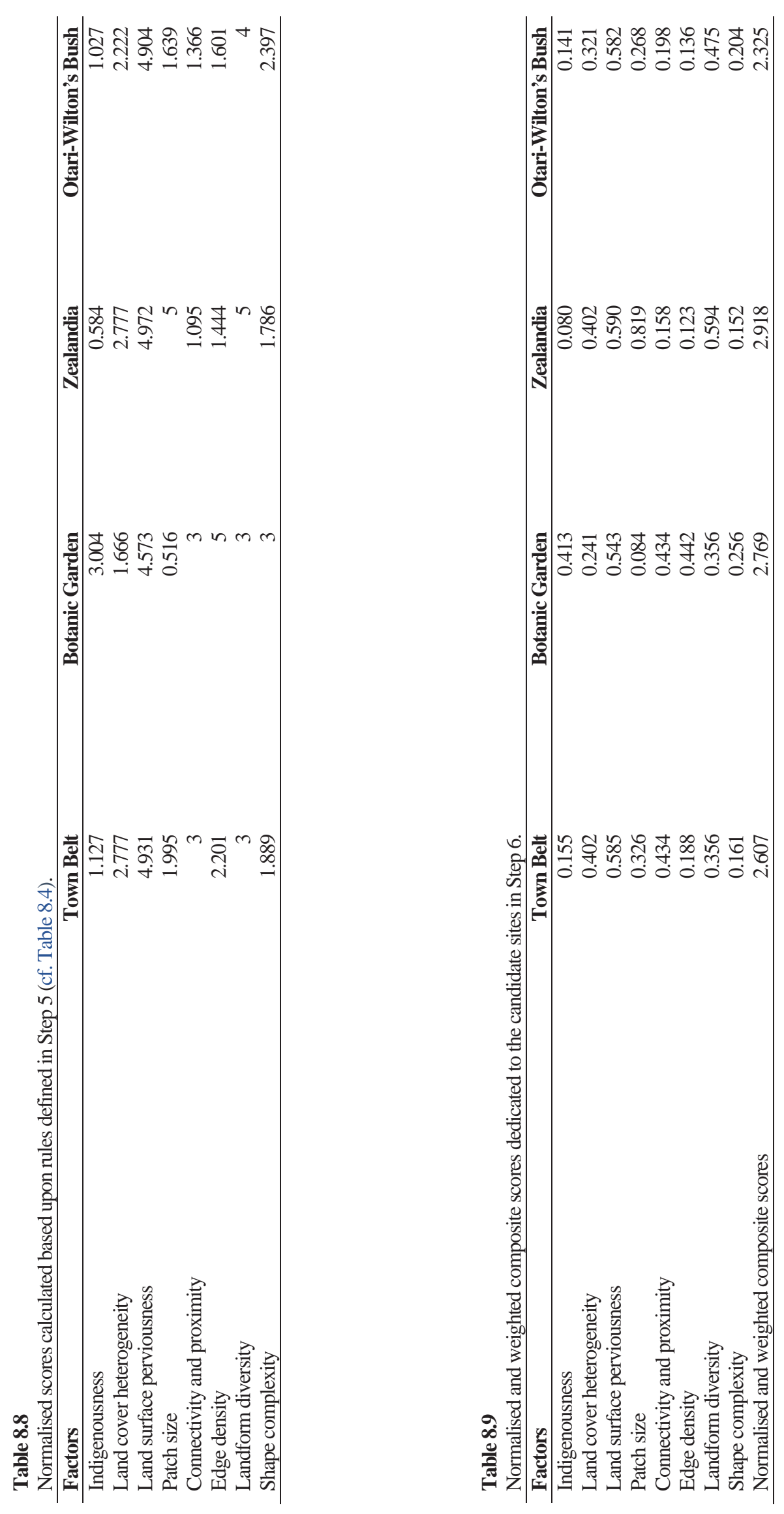


\subsection{DISCUSSION}

From a spatial perspective, this investigation confirms that Zealandia, Wellington's first urban wildlife sanctuary, is the most important site in urban Wellington to help safeguard indigenous biodiversity against dispersal of pest and disease under rising temperatures (Figure 8.4). This site plays a key role in supporting indigenous biodiversity not only in the Wellington urban landscape, but at the national level because this urban wildlife sanctuary harbours a wide range of vulnerable fauna including endangered and locally extinct species that can hardly survive in the wild (Cote et al., 2013; Watts et al., 2014; Beatley, 2016; Shaw and MacKinlay, 2016; Nelson et al., 2016). Unlike Botanic Garden, Otari-Wilton's Bush, and Town Belt, this site is currently fenced and is managed under effective predator control strategies.
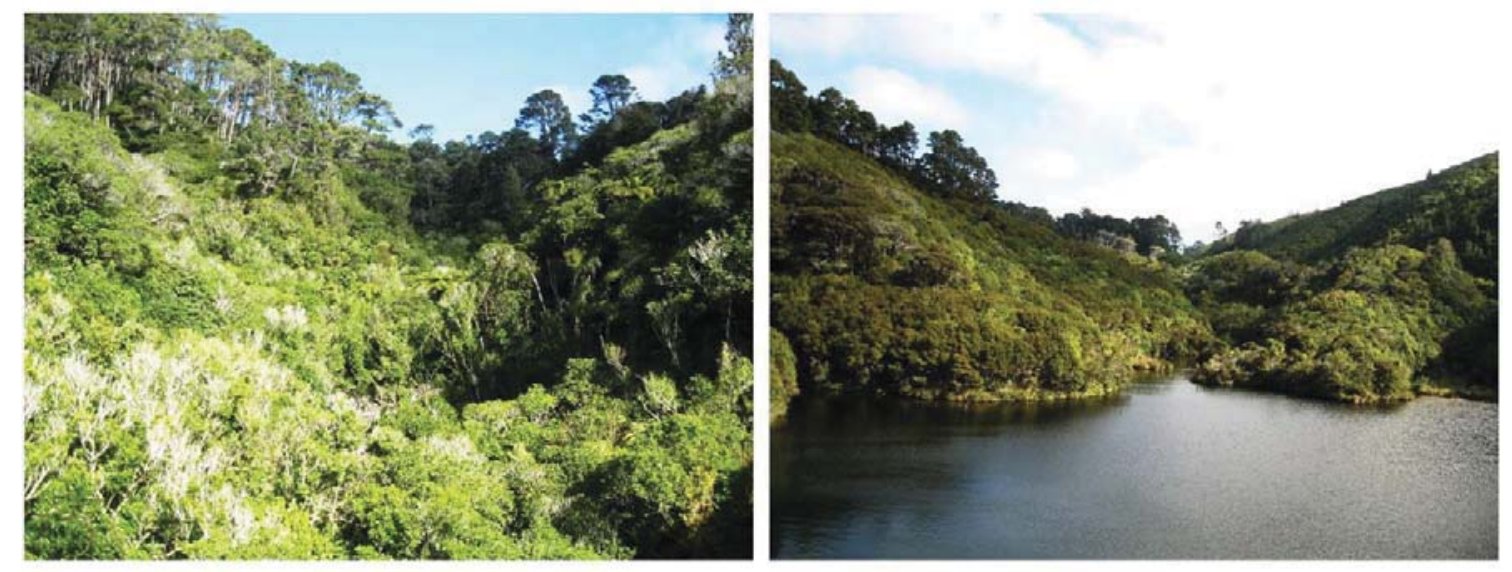

Figure 8.4. Expanses of indigenous flora associated with land cover heterogeneity and landform diversity in Zealandia (q.v. Appendix V).

Surprisingly, Botanic Garden, established in the 1840s (Shepherd and Cook, 1988), was identified as the second most important candidate site spatially capable of safeguarding vulnerable indigenous fauna in a changing climate (Figure 8.5). This can be regarded as a significant finding because Botanic Garden has the lowest value in terms of patch size compared to other candidate sites. Although patch size was ranked as the most important component of landscape pattern by international researchers and scientists (q.v. Section 2.4.2) and by New Zealand subject-matter experts (q.v. Section 3.3.1.1), and this is in agreement with the current literature (Forman and Godron, 1981; Forman, 1995; Dramstad et al., 1996; Cornelis and Hermy, 2004; Kang et al., 2015; Sing et al., 2016), this finding suggests that patch size should not be considered as the single most important factor for 
the site selection of urban wildlife sanctuaries because the collective importance of other factors may outweigh the significance of patch size.

This research does not suggest that Otari-Wilton's Bush and Town Belt are unimportant for safeguarding vulnerable indigenous fauna. The ranking presented in this research is completely relative. This means that the importance of each candidate site has been defined in relation to the other three sites. This analysis helps establish a scientific platform for the identification, ranking and prioritisation of candidate sites in terms of current compositions and configurations of patches of vegetation in highly fragmented landscapes. Such a platform helps decision makers to make appropriate decisions on the establishment of new pest-free urban wildlife sanctuaries though an informed site selection of the current patches of vegetation distributed in the study area. More importantly, the ecological importance of Otari-Wilton's Bush and Town Belt is confirmed because analysis shows that 62.41ha (76.75\%) and 38.37ha (38.83\%) of Otari-Wilton’s Bush and Town Belt respectively are covered by the class Broadleaved Indigenous Hardwoods, while only 1.43ha (5.58\%) of Botanic Garden is covered by this class at the moment (q.v. Table 8.6). If managed appropriately, the relatively large areas of patches covered by the class Broadleaved Indigenous Hardwoods in Otari-Wilton's Bush and Town Belt could potentially be replaced by the class Indigenous Forest through ecological succession processes (Davis and Meurk, 2001; Meurk and Hall, 2006; Williams, 2011; Wotton and McAlpine, 2013; q.v. Chapters 4 and 6; Appendix V). Therefore, from a long-term planning perspective, Otari-Wilton's Bush and Town Belt are two candidate sites of critical importance to serve as urban wildlife sanctuaries in the face of future climate change impacts, provided that they are managed appropriately over time. Similarly, 166.05 ha (67.06\%) of Zealandia is currently covered by the class Broadleaved Indigenous Hardwoods. Appropriate management of natural regeneration mechanisms could considerably increase the expanse of the class Indigenous Forest in this site. Despite this, Zealandia and Botanic Garden are currently the most important sites that should be managed for the conservation of indigenous biodiversity in the Wellington urban landscape, when urban biodiversity is addressed at the patch level and spatial isolation is required to avoid the spread of pests, diseases, and weeds.

Although Botanic Garden is shown to be a potentially suitable site for harbouring indigenous fauna at the present time, the site is heavily visited by international and local visitors. The site is not fenced at this time. The presence of people on the site can affect fauna by producing light and noise pollution, and common activities such as feeding them are associated with the risk of spread of diseases (Jones and Reynolds, 2008). Human food leftovers used by fauna as an alternative source of 
food are another challenge, reported by other researchers in other countries as a threat to urban biodiversity (e.g. Fernández-Juricic, 2001; Fernández-Juricic and Jokimaki, 2001). The presence of pets is also a problem to indigenous fauna when people visit this site. Thus, Botanic Garden can be considered as a socio-natural system in an urban context that deserves specific spatially-explicit land cover pattern design and management in order to ensure the coexistence of people and wildlife species in the long run. This means that not all areas covered by Botanic Garden are recommended to be fenced, but rather, areas of social and ecological importance should be separately managed in a way that minimises the negative impacts on indigenous biodiversity. The latter case requires further site specific research.
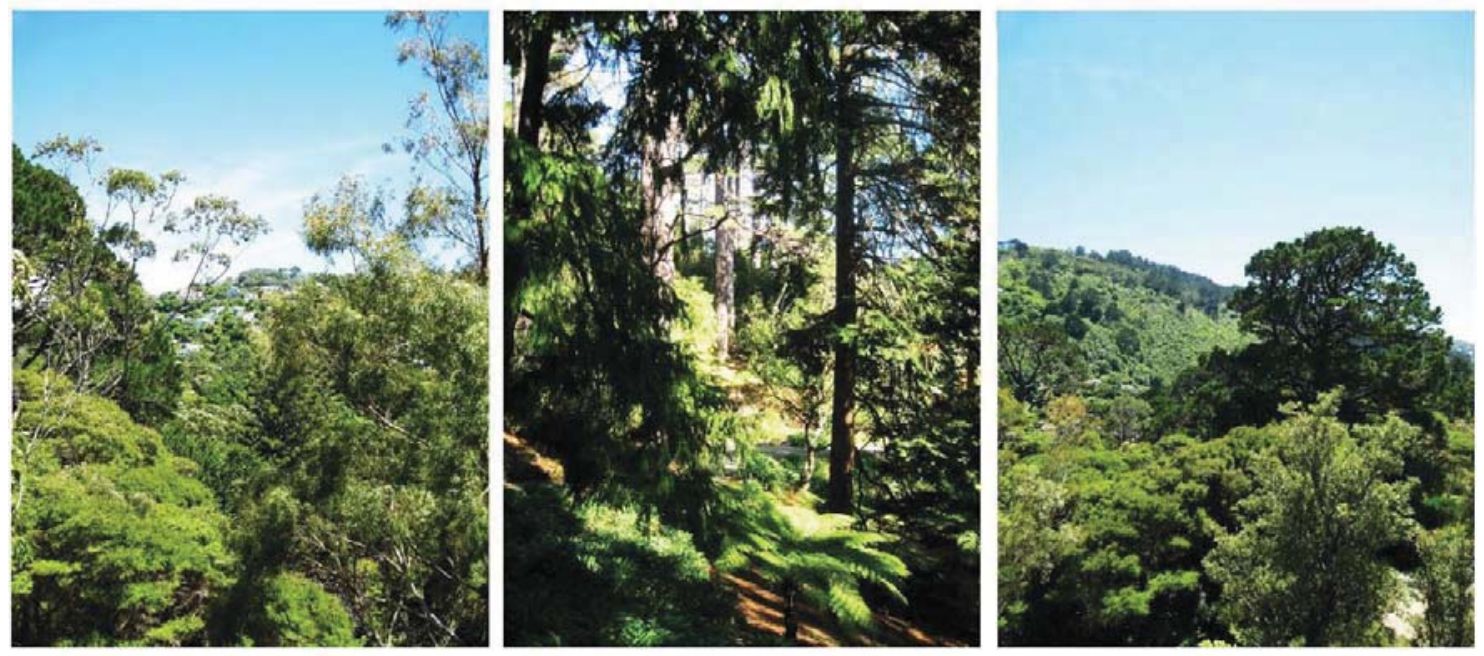

Figure 8.5. Expanses of indigenous flora associated with land cover heterogeneity and landform diversity in Botanic Garden (q.v. Appendix V).

In particular, the methodology designed and applied in this research paves the way to highlighting four key implications for landscape architecture and spatial planning in urban landscapes.

First, multi-criteria analysis of landscape pattern composition and configuration of patches of vegetation is a scientific-based, cost-effective, and relatively quick method to provide urban decision makers with a detailed spatial picture of opportunities for identifying and ranking apt locations as urban wildlife sanctuaries to help safeguard vulnerable indigenous fauna in urban landscapes, when negative impacts imposed by rising temperatures are predicted, such as increased predator pressures and the outbreak of diseases among fauna. 
Second, although very large patch size is thought by many to be a panacea for safeguarding biodiversity from climate change impacts in urban landscapes, as has already been suggested in the current literature and confirmed by surveys conducted in the course of this research (q.v. Chapters 2 and 3), patch size should not be regarded as the single most important factor in the process of site selection, because even small patches of vegetation may provide indigenous fauna with spatially suitable habitats in the face of climate change when habitat quality is met through other factors.

Third, it is particularly important to recognise the significance of some patches of vegetation that are capable of ecological succession through landscape restoration practices and/or natural regeneration mechanisms over time. Therefore, while the importance of patches of indigenous forest should not be overestimated for the site selection of urban wildlife sanctuaries, the importance of understanding the potential long-term role of some plant species in increasing the percentage of indigenousness in urban landscapes through the aforementioned processes should not be neglected.

Fourth, the presence of people in designed and semi-natural urban green spaces is inevitable. Where the rate of human-wildlife conflict of interests is high, however, a middle ground between societal demands and biodiversity requirements should be sought to minimise anthropogenic impacts on indigenous fauna while concurrently respecting cultural and social values ${ }^{57}$.

\subsection{CONCLUSION}

Biodiversity loss as a result of rapid urbanisation and climate change is increasingly reported by researchers, worldwide. Biological invasions by exotic species triggered by rising temperatures is a critical issue in urban New Zealand. New Zealand indigenous fauna are among taxonomic groups of high vulnerability to predator pressure (q.v. Section 1.1.5). In line with informed spatial planning for biodiversity conservation across urban landscapes, including what was recommended in Chapters 4 to 7, decision makers in urban New Zealand particularly need to recognise opportunities for safeguarding vulnerable indigenous fauna against the ill-effects of climate change, including informed decision-making regarding when and where isolated urban wildlife sanctuaries can be established most effectively to help avoid the spread of pests and weeds (cf. Sections 1.1.5 and 3.3.2). As shown spatially (q.v. Figures 8.1 and 8.3), there are opportunities for establishing new pest-free urban wildlife sanctuaries in the Wellington urban landscape. In line with other recommendations made in Study III (q.v. Chapters 4-8), despite widespread land cover change and dense human activities in the

\footnotetext{
${ }^{57}$ It is out of the scope of this research to explore and discuss possible ways throughout which the human-wildlife conflict is alleviated.
} 
Wellington urban landscape, this research reveals where, how, and why urban wildlife sanctuaries should be established in response to the local impacts of climate change. 


\section{CHAPTER 9 \\ Lessons learned and questions generated}

\subsection{INTRODUCTION}

This research is the first in New Zealand to address the role of the spatial patterning of patches of vegetation in safeguarding urban fauna against the local impacts of climate change by using a combined research methods and diverse data collection/analysis tools. The thesis ends with this chapter but concurrently this chapter can act as a foundation to drill down into the issues raised during the course of the research. The aims of this chapter therefore are to:

(1) Provide an overview of the key findings of the research;

(2) Articulate the most significant implications of the research for landscape architecture and land use planning through ten spatially-explicit recommendations

(3) State research limitations in a way that provides a deeper insight into barriers and deficits faced in the course of the research;

(4) Suggest six areas of inquiry for future research to address potentials for spatial planning for biodiversity conservation in a changing world.

\subsection{AN OVERVIEW OF THE KEY FINDINGS}

Today, urban landscapes grapple with on-going biodiversity loss. Climate change severely undermines the persistence of wildlife in human-occupied areas. Neither urbanisation nor climate change is slowing. As the urban population expands, open land, if not green spaces, will be inevitably dedicated to future housing development. While cities expand, landscapes fragment and ecosystems collapse. To respond to socio-economic needs and demands related to land and property markets, land cover change is a commonplace consequence of human interventions in urban landscapes. In this context, increasing expanses of green space for biodiversity conservation seem very unlikely. Thus, 
in-depth knowledge of the role of landscape patterns in safeguarding, or at least supporting, the remaining wildlife species against this unpredictable situation is critically necessary. This will help to safeguard or support wildlife without need for dedicating large areas to green spaces. In this research, particular emphasis has been placed on the existing patches of vegetation in the study area, rather than on increasing the percentage of green spaces. Through this strategy, it was revealed that informed interventions in the spatial composition and configuration of the current patches of vegetation can contribute to biodiversity conservation in an era of climate change provided that key ecological processes such as habitat use, species-ecosystem mutualisms, predator-prey interactions, seed dispersal patterns, natural regeneration mechanisms, and successional processes are deeply understood and, accordingly, taken into account.

This research was designed to address the question: 'what is an optimised landscape pattern composition and configuration capable of safeguarding indigenous fauna against the local impacts of climate change in a highly human-occupied urban landscape?' The term optimised landscape pattern was intentionally used in this research because having fully intact natural ecosystems in urban landscapes is likely to be impossible in practice. Three studies were conducted to scrutinise the research question. Detailed responses have been articulated in the discussion and conclusion sections of each chapter reporting Studies I, II, and III (Chapters 4-8).

As an overview of the most important findings of this research, in Study I, the most important components of landscape pattern affecting the presence, richness, and abundance of urban fauna in an era of climate change were identified, ranked, and discussed. According to Study I, patch size, connectivity and proximity and land cover heterogeneity are believed to be respectively the most important factors affecting the overall level of urban biodiversity with particular emphasis on avifauna, small mammals, and reptiles. This study also revealed that indigenousness is the next most important factor in urban landscapes of the Southern Hemisphere (q.v. Chapter 2). In Study II, the results derived from Study I were adjusted and confirmed to be applicable to a New Zealand context. In Study II, six New Zealand subject-matter experts provided valuable information about the current biodiversity challenges and opportunities in a way that contributes to informed spatial planning for biodiversity conservation in urban New Zealand under a changing climate (q.v. Chapter 3). In Study III, a comprehensive spatial analysis of landscape pattern composition and configuration in the Study Area was undertaken in three levels of landscape, class, and patch using GIS and FRAGSTATS (q.v. Chapters 4-8). This study revealed that the Study Area consists of sixteen land cover classes, which shows that in terms of land cover heterogeneity, the study area is a rich matrix of patches of 
vegetation. Analysis showed that approximately $60 \%$ of the study area is covered by different types of green spaces. Thus, in terms of land surface perviousness, the study area provides suitable conditions for biodiversity conservation, as a whole. But in terms of indigenousness, the study area is comprised of four indigenous land cover classes cumulatively covering less than 25\% of the study area. Even worse, less than 1\% (121.7 ha) of the study area is covered by the class Indigenous Forest, which is thought to be the primary habitat of a wide range of indigenous fauna in urban New Zealand (q.v. Chapter 4). In terms of landform diversity, the study area is highly diverse. The research, however, demonstrates that $9.45 \%$ of the study area is covered by south-facing slopes, which is believed to be the most suitable aspect for biodiversity conservation goals in the Southern Hemisphere in the face of warmer than average temperatures. Of this extent, only 9.13 ha ( $0.06 \%$ of the study area) overlap the class Indigenous Forest (q.v. Chapter 5).

In terms of patch size, shape complexity, and edge density, the current composition and configuration of patches of different classes are not effectively capable of providing suitable conditions for urban fauna against the ill-effects of climate change. For example, this research found that only one-third of expanses covered by the class Indigenous Forest can serve as interior habitats that are vital to providing urban fauna with safe zones against edge effects. Conversely, the number of exotic patches of vegetation, as well as spatial distance between them, can increase the risk of pest and weed dispersal under a changing climate. In terms of connectivity and proximity, the spatial distance between patches of indigenous forest is not suitable for facilitating the process of seed dispersal by the selected keystone species. There are, however, opportunities to overcome this deficit (q.v. Chapters 6 and 7). For example, there are still great opportunities for landscape restoration through natural regeneration mechanisms. Areas covered by the classes Broadleaved Indigenous Hardwoods and Gorse and/or Broom are potentially capable of succession towards the class Indigenous Forest. This research revealed that respectively 416.14 ha (13.4\% of the class) and 189.65 ha (11.2\% of the class) of these classes overlap south-facing slopes and therefore, are strategically significant for landscape restoration practices (q.v. Chapters 5 and 6). In addition, the research specified strategic locations for establishing stepping stones in order to compensate for the lack of suitable spatial connectivity and proximity between the existing patches of the class Indigenous Forest (q.v. Chapter 7). To respond to the ongoing risk of pest and weed dispersal, four candidate sites covering the largest patches of indigenous forest were studied, spatially analysed and ranked, as potential pest-free urban wildlife sanctuaries against the negative effects of climate change (q.v. Chapter 8). In Study III, it was revealed that the western part of the Wellington urban landscape is potentially the most strategic area of ecological significance for biodiversity conservation through spatial planning (q.v. Chapters 4-8). 


\subsection{IMPLICATIONS FOR LANDSCAPE ARCHITECTURE}

In addition to the detailed information provided in the discussion and conclusion sections of Chapters 2 to 8, ten recommendations arise from the research that can effectively help achieve a more optimised landscape pattern composition and configuration that reverses the current biodiversity loss in urban New Zealand in general and the Wellington urban landscape in particular ${ }^{58}$. These recommendations provide single important opportunities for biodiversity conservation through spatial planning in the study area (Figure 9.1):

(1) Areas covered by the class Indigenous Forest should be strategically preserved as primary habitats of indigenous fauna as well as sources of the seeds of indigenous flora to support natural regeneration mechanism;

(2) Expanses covered by the class Indigenous Forest should be increased through natural regeneration mechanisms; spatial planning for biodiversity conservation in urban New Zealand should place particular emphasis on informed interventions in the spatial composition and configuration of the current patches of vegetation in a way that optimises patch size, shape complexity, and connectivity and proximity. Informed interventions are necessary to make sure that the study area involves enough interior habitats, and that the spatial distance between patches of vegetation is not more than $100 \mathrm{~m}$. This research does not recommend a spatial threshold for the area of interior habitats, but strongly recommends strategies that reconfigure patches of the class Indigenous Forest through re-vegetation and/or natural regeneration mechanisms in order to decrease the extent of edge areas;

(3) Areas covered by the class Indigenous Forest overlapping with south-facing slopes can be regarded as safer places in the face of rising temperatures compared to other aspects of slope. These areas should be preserved, in particular against future urban expansion and development;

(4) Areas covered by the classes Broadleaved Indigenous Hardwoods and Gorse and/or Broom, specifically those that overlap south-facing slopes, should be strategically managed in a way

\footnotetext{
58 These recommendations arose from a wide range of data generated during the course of the research and are presented in numerous tables and maps (q.v. Chapters 2-8). As original sources of spatial data used in this research varied in nature (i.e. scale, resolution, content, etc.), it was not possible to create a single map of the study area to depict all opportunities for biodiversity conservation through spatial planning. The audience, however, can strategically use specific tables and maps depending on the nature of information required.
} 
that facilitates the process of ecological succession towards the class Indigenous Forest in the coming decades. Patches of vegetation with lower edge area should take precedence for landscape restoration practices because they are less prone to damage from extreme weather events and accordingly, will provide indigenous fauna with enough space as interior habitats;

(5) Areas covered by exotic patches of vegetation should be spatially managed in a way that reduces the risk of pest and weed dispersal. The fewer the number of patches of exotic vegetation, the less risk for seed and pest dispersal. Smaller patches with lower edge density and connectivity and proximity are also desirable to achieve this goal;

(6) The western part of the Wellington urban landscape can be considered as the most important hub of ecological significance for biodiversity conservation against climate change. This hub consists of the largest patches of indigenous forest and is potentially capable of providing a stronghold for indigenous fauna in the face of extreme weather events;

(7) In the western part of the Wellington urban landscape, a spatial distance of $<100$ m between patches of indigenous forest is not currently available on the ground. To compensate for the lack of the desired connectivity and proximity, stepping stones should be strategically established between potential seed sources and deposit sites to facilitate species movement and provide additional food sources for pollination and seed dispersal, and consequently, contribute to natural regeneration over time. Ecologically strategic plant species that can serve best for this purpose, as well as the potential location for establishing these stepping stones, were specified in detail;

(8) Irrespective of the importance of open patches of vegetation distributed across the landscape, fenced pest-free urban wildlife sanctuaries are also required to respond to unpredictable impacts of climate change in the future more specifically for those species that are more vulnerable to climate change. Zealandia, Botanic Garden, Town Belt and Otari-Wilton's Bush are respectively the most suitable areas for safeguarding the most vulnerable urban fauna against uncertainties associated with climate change;

(9) Future urban expansions including housing and road development should occur in expanses of exotic land cover classes with the least ecological significance in order to ensure the survival of important patches of vegetation; 
(10) Infill development is inevitably needed to avoid further urban expansion over areas of ecological significance. Even urban development in green spaces of the least ecological importance may be detrimental to biodiversity because it will reduce at least the rate of land surface perviousness, if not other components of landscape pattern, in the study area.

As shown in Study III (q.v. Chapters 4-8), these recommendations are achievable and now measurable, given that this research has provided a scientific baseline for others to examine the study area and measure the degree to which land cover patterns will undergo change over time ${ }^{59}$. This can be measured in terms of the eight principal components of landscape pattern as major indicators, spatial analysis techniques and tools, and the core set of landscape metrics used in the course of this research. The Precautionary Principle (q.v. Chapter 1) should be taken into consideration when applying these recommendations in practice.

${ }^{59}$ A wide range of spatial data provided on tables and maps can be used for this purpose. 


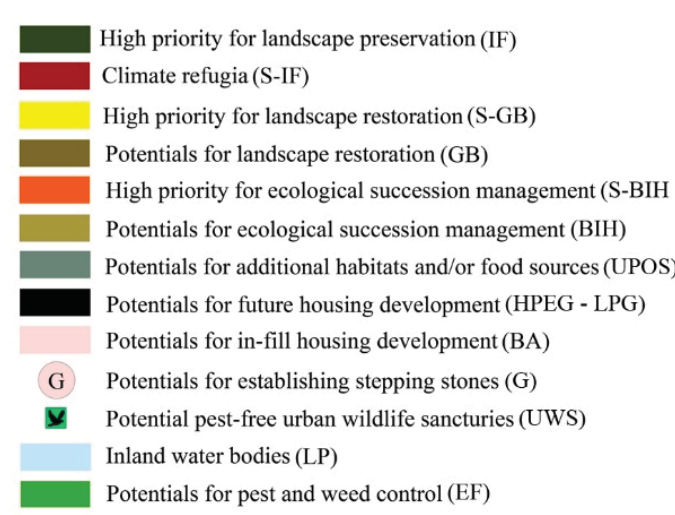

Figure 9.1. An example of results derived from the overlay analysis revealing applicable opportunities for biodiversity conservation through spatial planning in the western part of the Wellington urban landscape - the ecological hub (q.v. Chapters 5, 6, and 7). This map should be interpreted in relation to information provided in Study III (q.v. Chapters 4-8; cf. Figures 4.1, 5.3, 5.4, 5.5, 5.6, 6.4, 6.5, 6.6, 7.3, 7.7, 7.9, 8.1, and 8.3).

Areas covered by the class Indigenous Forest (IF) should be considered as high priority landscape preservation sites (q.v. Chapters 3 and 4). South-facing slopes covered by this class (S-IF) also can serve as climate refugia for urban wildlife in the face of rising temperatures (q.v. Chapter 5). South-facing slopes covered by the class Gorse and/or Broom (S-GB) should be regarded as sites for high priority landscape restoration though natural regeneration mechanisms (q.v. Chapter 6). South-facing slopes covered the class Broadleaved Indigenous Hardwoods (S-BIH) should be managed for ecological succession towards the class Indigenous Forest (q.v. Chapters 4, 5, and 6). Other aspects of slopes covered by these two classes (GB and BIH) should be concurrently managed for long-term landscape restoration practices (q.v. Chapters 4, 5 and 6). Pest and weed control should particularly be focused on areas covered by the class Exotic Forest (EF) (q.v. Chapters 4 and 7). The existing spatial gaps (G) between patches of the classes Indigenous Forest (IF), Gorse and/or Broom (GB), and Broadleaved Indigenous Hardwoods (BIH) should be bridged through establishing stepping stone patches in the built environment (q.v. Chapter 7). This can be attainable using informed interventions in very small patches of vegetation including revegetation in private backyards, establishing new woodlots with enough canopy trees, green roofs, constructed wetlands, small green spaces in impervious areas such as car parks, etc. Areas covered by the class Urban Parkland/Open Space (UPOS) can be revegetated and/or spatially managed to serve more effectively as additional habitat/food source. Future housing development should occur in areas of the least ecological significance to biodiversity or sites covered by the classes High Producing Exotic Grassland (HPEG) and Low Producing Grassland (LPG) (q.v. Chapters 4 and 7). In areas covered by the class Built-up Area (BA), in-fill development is also an effective option to minimise development impacts on biodiversity (q.v. Chapter 7). The most strategic sites capable of serving as urban wildlife sanctuaries (UWS) are located in the western part of the Wellington urban landscape (q.v. Chapter 8). These sites can be considered as pest-free havens for vulnerable urban fauna in the face of increased predator pressures. Areas covered by the class Lake and/or Pool (LP) should be appropriately be managed in relation to areas covered by the class Indigenous Forest (IF).

\subsection{RESEARCH LIMITATIONS}

Due to the nature of this research, which used spatial data to address biodiversity issues in urban landscapes in an era of climate change, the research faced specific limitations. Details are provided in the following sections. 


\subsubsection{Study I}

Although recent advancements in technological equipment have provided opportunities to study the species-ecosystem interactions in urban landscapes, little has been known prior to this research about the role of components of landscape pattern in enhancing the presence, richness, and abundance of urban fauna under a climate that continues to change. Responses received from eightyseven international researchers in Study I were based upon long-term experience in the field, but at the same time, were subjective. Although the convergence of opinions regarding the topics under discussion as well as consistency between their responses and the literature were two significant signs that validate the accuracy of results, further research is still required to understand potential interactions between urban fauna and land cover patterns under the changing climate.

\subsubsection{Study II}

While New Zealand has been, and still is, a leading country in urban biodiversity research and practice since the twentieth century, and although the ratio of the number of publications in this field to the total population of the country is very high compared to many nations, it was difficult to find subject-matter experts with track records of relevant experience on spatial dimensions of biodiversity conservations in urban landscapes under climate change. Approximately six months were spent locating and then discussing the research with six participants who agreed to take part in interview sessions.

\subsubsection{Study III}

Similar to standard GIS-based spatial analysis practices, this research is subject to an acceptable level of spatial error. The level of error is extremely low. Different methods were applied in the course of this research to increase the accuracy of the research in the spatial analysis of land cover patterns in the study area. The accuracy of basic spatial data was checked at different stages of the research using Google Earth satellite imageries. In some cases, field surveys were also conducted to reduce the error level in mapping, and accordingly, spatial analysis processes. Although groundtruthing was undertaken in certain areas to make sure that basic spatial data related to land cover classes reflects what actually does exist on the ground, it was not possible to undertake a more comprehensive and in-depth field survey of the entire study area to check potential errors in the available data due to the lack of time and sufficient funds. In some topographically diverse areas where access was not easily available, especially in deep valleys and steep rocky hills covered by 
dense vegetation, helicopters and/or drones are ideally required to make more reliable and detailed observations.

\subsection{AREAS OF INQUIRY FOR FUTURE RESEARCH}

Based upon a three-year research period on the importance of spatial planning for biodiversity conservation in urban landscapes (2015-2018), the final section of this chapter reports the current knowledge gaps in the field with particular attention to what is needed to help safeguard urban fauna against climate change. Different in nature and scale, research areas are categorised into six key topics (q.v. Sections 9.5.1-9.5.6). The point of unity in these topics is the specific emphasis on the importance of a spatially-explicit perspective for addressing biodiversity issues in a changing environment. There is emerging insight into the importance of research on species behaviour in space and time in relation to land cover classes in an era of climate change (Beever et al., 2017). Although significant and promising, it is necessary for this trend to extend to land cover patterns to generate spatially-explicit knowledge for biodiversity conservation in a changing world where urban growth confines, if not engulfs, the chance of increasing expanses of existing wildlife habitats in and outside urban landscapes.

\subsubsection{Long-term land cover change analysis}

Spatial information, produced and depicted in maps, tables, and charts, provides a scientific baseline for future research in the topic. This baseline enables other researchers to undertake similar research to examine the degree to which landscape patterns are changing over time and how potential changes should be interpreted in an era of climate change. This will enable policy makers to have a clear picture of what they need to do to safeguard indigenous biodiversity through spatial planning and informed interventions in the current patterns. The trajectory of changes can be recorded using GIS.

Spatial data must be constantly updated. Otherwise, the data reliability will reduce over time. Not only useful for typical land use/land cover planning and management practices, long-term data on land cover change will provide a robust platform for undertaking alternative futures and scenario analysis to envision spatially-explicitly landscape futures. This could enable a wider range of stakeholders to make more informed decisions on biodiversity and wildlife management in humanoccupied landscapes (Hulse et al., 2000; Baker et al., 2004; Rastandeh, 2015; Hulse et al., 2016). 


\subsubsection{Technology-equipped field survey}

Field research is essential using Global Positioning System (GPS) telemetry to study the behaviours of the species of interest in space and time in relation to the spatial composition and configuration of land cover patterns, in order to reveal more empirical data for future research. GPS telemetry, however, requires an informed understanding of ecological traits and niches of species of interest as well as biodiversity conservation goals (Latham et al., 2015). Although telemetry-based methods are not new in wildlife management research (Aebischer et al., 1993), little research has been conducted globally on avifauna, small mammals, and reptiles in urban landscapes under a changing climate. Telemetry-based methods enable researchers to develop a higher level of knowledge on the spatial ecology of urban fauna and species-ecosystem interactions in a changing context. Considering this aim, initial steps have recently been taken towards developing a higher level of knowledge on habitat use and in relation to land cover classes in urban landscapes (Braaker et al., 2017; Hämäläinen et al., 2018). The role of land cover pattern (e.g. patch size, edge density, etc.), however, needs to be scrutinised in more depth. In particular, thanks to recent advancements in micro-GPS technology, GPS telemetry of New Zealand avifauna is no longer impossible.

\subsubsection{Spatial ecology of exotic fauna}

While the negative effects of introduced mammals should seriously be taken into account to ensure the continuation and survival of indigenous biodiversity over time in urban New Zealand, research should be undertaken to investigate why introduced mammals cannot provide ecosystem services similar to the services provided where they are identified as native species ${ }^{60}$. For example, research on the occurrence and density of introduced mammals in urban landscapes, as has been conducted in urban England (Bonnington et al., 2014), is also necessary in urban New Zealand. Furthermore, it is also necessary to develop an understanding of how exotic species can be spatially managed in a way that reduces potential impacts on indigenous biodiversity ${ }^{61}$. In New Zealand, research has started to use GPS telemetry on some exotic fauna such as the European hedgehog (Recio et al., 2011; 2013) and feral cat (Recio et al., 2014) in non-urban landscapes. This device also can facilitate the study of the spatial ecology of exotic fauna in urban New Zealand under different climate change scenarios.

\footnotetext{
${ }^{60}$ Specifically in the case of New Zealand, exotic species introduced to the country from the UK and Australia are of ecological significance in their home country (q.v. Chapter 1).

${ }^{61}$ Research is in progress to study the spatial ecology of grey squirrel (Sciurus carolinensis) in the UK. In the UK, grey squirrel is classified as an exotic species. This species was imported to the UK from the United States of America where the species plays a key role in providing a range of ecosystem services. The grey squirrel in the UK, however, is extremely harmful to native biodiversity (q.v. Stevenson et al., 2013).
} 


\subsubsection{Predator-prey interactions}

Some of those mammals that are recognised as exotic in New Zealand have been radiotracked in urban Australia in relation to their interactions with various land cover classes and their food preferences (Harper, 2006). In order to optimise the spatial composition and configuration of habitats to ensure the survival of indigenous fauna, predator-prey interactions should be studied in relation to space and time. Although there are examples of research in this area in urban New Zealand (e.g. van Heezik et al., 2010), future research is needed particularly with a focus on habitat edges. As discussed, the ill-effects of climate change including the risk of predator-prey interactions are higher along edges of habitat patches (q.v. Chapters 3 and 7). Long-term monitoring can also be carried out using different tools such as motion-triggered and nest cameras as recently conducted by Anton et al. (2018) in the Wellington urban landscape to monitor the presence of exotic mammals, as well as video-recording techniques to monitor the behaviour of indigenous avifauna in response to exotic mammals and how spatial locations and land cover patterns may influence these interactions.

\subsubsection{Citizen science: an alternative source of information}

Until sufficient empirical data is available, citizen science should be considered as an alternative source of information to compensate for the lack of accurate scientific data. Chambers et al. (2017) underscore the significance of citizen science in biodiversity research under climate change in the Southern Hemisphere, where data is still insufficient compared to the Northern Hemisphere. Using citizen science has recently been suggested as an alternative way to gather spatial data relating to exotic predator mammals (Kikillus et al., 2017) and the estimation of indigenous bird abundance (van Heezik and Seddon, 2017) in urban New Zealand. Although subject to a relatively higher level of error compared to some other methods, observations made by people and their ongoing participation in urban biodiversity projects may help to contribute to the knowledge of species behaviour in space and time. Areas and times of presence, habitat use, movement, nesting sites, and foraging behaviours can be easily reported by the general public. Information obtained can next be digitalised and spatialised using GIS in order to address potential relationships between reported locations of species and land cover classes dominated in those locations with reference to the scale of time. This information can also be linked to land cover patterns. One of the disadvantages of this method of data collection is a relatively low level of confidence and data accuracy. The method is, however, relatively quick and cost-effective because it does not require advanced equipment or hightechnology. This, undoubtedly, requires a large number of interested and relatively informed people 
who believe deeply in the importance of engaging in such participatory projects. According to research conducted by Snep et al. 2016, such a social context is available in urban New Zealand.

\subsubsection{Urban-natural interactions: planning for a wider network}

Ecological and administrative boundaries are not necessarily identical. Researchers, however, have to specify the boundary of their research sites considering a spectrum of criteria including environmental, social, political, and administrative factors in order to enable policy makers to take advantages of the research outputs. In practice, however, fauna interact with areas outside urban landscapes. Therefore, the ecological integrity and quality of areas outside urban landscapes are as important as that of patches of vegetation distributed inside urban landscapes. Thus, building up an understanding of species' movement between urban and non-urban landscapes is needed to provide key information for biodiversity conservation through spatial planning at a broader scale. Recent research shows that even where areas of native habitats are relatively abundant, adjacent urban landscapes play a pivotal role in harbouring endangered fauna during winters (Jokimäki et al., 2017). In urban landscapes, where human land use activities meet natural ecosystems (i.e. the urban-wildland interface - Radeloff et al., 2005) land cover patterns must be spatially designed and managed in a proper manner. This is because in transitional zones along these areas, species' richness, the level of predator-prey interactions, and the rate of human-wildlife conflicts are potentially higher and patterns inevitably influence these processes. As urban landscapes are being expanded, the risk of ecosystem degradation and habitat loss increases and the chance of human-wildlife conflict (Soulsbury and White, 2016) also consequently rises in the urban-wildland interface. Thus, urban landscapes should also be studied as a highly dynamic and complex component of a greater natural-social system. This level of future research and analysis requires advanced equipment, accurate and high resolution spatial data, and sufficient funds for a long-term, comprehensive, and in-depth field survey. While examples of such advanced studies are gradually emerging in the literature (Gallo et al., 2017; Murray and St Clair, 2017; Threlfall et al., 2017), nevertheless, current knowledge is still very limited.

\subsection{CONCLUSION}

Amid a plethora of political-economic conflicts, the world's inhabitants are mostly oblivious to the nature of unprecedented threats resulting from widespread biodiversity loss. Urban regions are most often situated at ecosystem junctions, and as cities expand, valuable ecosystems and wildlife habitats collapse irreversibly, and ecosystem services decrease substantially. As complex patternprocess relationships, one change leads irrevocably to another in a series of both positive and negative 
feedback loops. In such a complex context, climate change imposes extra stress on ecosystems, wildlife, and people. The ultimate outcome for future generations might well be the loss of thousands of vital species, and ultimately even human extinction.

To reverse, or at least curtail this threatening trend, an in-depth knowledge of pattern-process relationships must be deeply understood and strategically applied in real world urban environments. Stakeholders who have a voice in the process of decision-making must be informed about the importance of safe-guarding the most strategic pattern-process relationships. Humans are now fundamental participants in urban and non-urban environmental pattern-processes. If these patterns are managed and implemented appropriately, the coexistence of humans and wildlife can help environmental sustainability in urban landscapes.

As a highly biodiverse and urbanised context, New Zealand provides an important case study for this investigation. Research shows how the spatial design of urban land cover patterns - with particular attention to the habitat requirements of fauna and other key ecological processes in urban landscapes - may contribute to biodiversity conservation. The research argues that a deep knowledge of keystone species' behaviours in space and time is required and should be an integral part of landscape architecture commitments to alleviate the current biodiversity challenges. When this knowledge enters into the process of decision-making, essential environmental patterns can be effectively safeguarded or re-established.

This research helps to establish a platform in order for researchers and practitioners to show how this overarching goal may be attained. It also challenges the existing boundaries of landscape architecture and designates new strategic roles for landscape architecture researchers, urban policymakers, and practitioners who address land-related issues under a climate that continues to change. 


\section{REFERENCES}

Abdrabo, M. A., \& Hassaan, M. A. (2015). An integrated framework for urban resilience to climate change - Case study: Sea level rise impacts on the Nile Delta coastal urban areas. Urban Climate, 14, 554-565.

Abrahamson, M. (2004). Global cities. New York: Oxford University Press.

Abunnasr, Y. F. (2013). Climate change adaptation: A green infrastructure planning framework for resilient urban regions. $\mathrm{PhD}$ Dissertation: University of Massachusetts at Amherst.

Ackerly, D. D., Loarie, S. R., Cornwell, W. K., Weiss, S. B., Hamilton, H., Branciforte, R., \& Kraft, N. J. B. (2010). The geography of climate change: implications for conservation biogeography. Diversity \& Distributions, 16(3), 476-487.

Adams, C. E. (2016). Urban wildlife management. Boca Raton: CRC Press.

Aebischer, N. J., Robertson, P. A., \& Kenward, R. E. (1993). Compositional analysis of habitat use from animal radio-tracking data. Ecology, 74(5), 1313-1325.

Aguilar, G. D., Farnworth, M. J., \& Winder, L. (2015). Mapping the stray domestic cat (Feliscatus) population in New Zealand: Species distribution modelling with a climate change scenario and implications for protected areas. Applied Geography, 63, 146-154.

Ahern, J. (1999). Spatial concepts, planning strategies and future scenarios: A framework method for integrating landscape ecology and landscape planning. In: Klopatek J. \& Gardner, R., (Eds.), Landscape ecological analysis: issues and applications, (pp. 175-201), New York, Springer.

Ahern, J. (2013). Urban landscape sustainability and resilience: the promise and challenges of integrating ecology with urban planning and design. Landscape Ecology, 28(6), 1203-1212.

Aida, N., Sasidhran, S., Kamarudin, N., Aziz, N., Puan, C. L., \& Azhar, B. (2016). Woody trees, green space and park size improve avian biodiversity in urban landscapes of Peninsular Malaysia. Ecological Indicators, 69, 176-183.

Alberti, M. \& Marzluff, J. M. (2004). Ecological resilience in urban ecosystems: linking urban patterns to human and ecological functions. Urban Ecosystems, 7(3), 241-265.

Alberti, M. (2005). The effects of urban patterns on ecosystem function. International Regional Science Review, 28, 168192.

Allen, R. B., \& Lee, W. G. (2006). Biological invasions in New Zealand. Heidelberg: Springer.

Alvey, A. A. (2006). Promoting and preserving biodiversity in the urban forest. Urban Forestry \& Urban Greening, 5(4), 195-201.

Amo, L., Galvan, I., Tomas, G. \& Sanz, J. J. (2008) Predator odour recognition and avoidance in a songbird. Functional Ecology, 22, 289-293.

Anderson, S. H., Kelly, D., Ladley, J. J., Molloy, S., \& Terry, J. (2011). Cascading effects of bird functional extinction reduce pollination and plant density. Science, 331(6020), 1068-1071.

Anderson, S. H., Kelly, D., Robertson, A. W., Ladley, J. J., \& Innes, J. G. (2006). S04-3 birds as pollinators and dispersers: a case study from New Zealand. Acta Zoologica Sinica, 52(1), 112-115. 
Andersson, E., Barthel, S., Borgström, S., Colding, J., Elmqvist, T., Folke, C., \& Gren, Å. (2014). Reconnecting cities to the biosphere: stewardship of green infrastructure and urban ecosystem services. Ambio, 43(4), 445-453.

Andersson-Sköld, Y., Bergman, R., Johansson, M., Persson, E., \& Nyberg, L. (2013). Landslide risk management - A brief overview and example from Sweden of current situation and climate change. International Journal of Disaster Risk Reduction, 3, 44-61.

Andren, H. (1994). Effects of habitat fragmentation on birds and mammals in landscapes with different proportions of suitable habitat: a review. Oikos, 71(3), 355-366.

Anton, V., Hartley, S., \& Wittmer, H. U. (2018). Evaluation of remote cameras for monitoring multiple invasive mammals in New Zealand. New Zealand Journal of Ecology, 42(1), 74-79.

Aronson, M. F., La Sorte, F. A., Nilon, C. H., Katti, M., Goddard, M. A., Lepczyk, C. A., ... \& Dobbs, C. (2014). A global analysis of the impacts of urbanization on bird and plant diversity reveals key anthropogenic drivers. Proceedings of the Royal Society of London B: Biological Sciences, 281(1780), 20133330.

Asgarian, A., Amiri, B. J., \& Sakieh, Y. (2015). Assessing the effect of green cover spatial patterns on urban land surface temperature using landscape metrics approach. Urban Ecosystems, 18(1), 209-222.

Atlan, A., Udo, N., Hornoy, B., \& Darrot, C. (2015). Evolution of the uses of gorse in native and invaded regions: what are the impacts on its dynamics and management?. Revue d'Ecologie, Terre et Vie, 70 (supplément 12-Espèces nvasives).

Ausseil, A. G. E., Kirschbaum, M. U., Andrew, R. M., McNeill, S., Dymond, J. R., Carswell, F., \& Mason, N. W. (2013). Climate regulation in New Zealand: contribution of natural and managed ecosystems. In: Dymond, J. R., (Ed.), Ecosystem services in New Zealand - conditions and trends, (pp. 386-399), Lincoln: Manaaki Whenua Press.

Baber, M. A. T. T., Brejaart, R., Babbitt, K. I. M. B. E. R. L. Y., Lovegrove, T., \& Ussher, G. R. A. H. A. M. (2009). Response of non-target native birds to mammalian pest control for kokako (Callaeas cinerea) in the Hunua Ranges, New Zealand. Notornis, 56, 176-182.

Bagnall, R. G. (1979). A study of human impact on an urban forest remnant: Redwood Bush, Tawa, near Wellington, New Zealand. New Zealand Journal of Botany, 17, 117-126.

Baillie, S. M., \& Brunton, D. H. (2011). Diversity, distribution and biogeographical origins of Plasmodium parasites from the New Zealand bellbird (Anthornis melanura). Parasitology, 138(14), 1843-1851.

Baker, A. (1999). Food plants of Bellbird (Anthornismelanttra), Tūī (Prosthemaderanovaeseelandiae) and New Zealand Pigeon (Hemiphaganovaeseelattdiae) in Dunedin. Notornis, 46, 270-272.

Baker, J. P., Hulse, D. W., Gregory, S. V., White, D., Van Sickle, J., Berger, P. A., ... \& Schumaker, N. H. (2004). Alternative futures for the Willamette River basin, Oregon. Ecological Applications, 14(2), 313-324.

Balvanera, P., Pfisterer, A. B., Buchmann, N., He, J. S., Nakashizuka, T., Raffaelli, D., \&Schmid, B. (2006). Quantifying the evidence for biodiversity effects on ecosystem functioning and services. Ecology Letters, 9(10): 1146-1156.

Barbaro, L., Brockerhoff, E. G., Giffard, B., \& van Halder, I. (2012). Edge and area effects on avian assemblages and insectivory in fragmented native forests. Landscape Ecology, 27(10), 1451-1463.

Barnes, T. G (2000). Landscape ecology and ecosystems management. University of Kentucky College of Agriculture, Cooperative Extension Service. Frankfort: Kentucky State University.

Bashford, A. (2014). Global population: history, geopolitics, and life on earth. New York: Columbia University Press.

Beatley, T. (2016). Wellington, New Zealand: From Town Belt to Blue Belt. In: Beatley, T., (Eds.), Handbook of Biophilic City Planning and Design, (pp. 75-84), Washington: Island Press. 
Beever, E. A., Hall, L. E., Varner, J., Loosen, A. E., Dunham, J. B., Gahl, M. K., ... \& Lawler, J. J. (2017). Behavioral flexibility as a mechanism for coping with climate change. Frontiers in Ecology \& the Environment, 15(6): 299-308.

Bell, R. (1996). Seed Dispersal by kererū (Hemiphaganovaeseelandiae) at Wenderholm Regional Park. MSc Thesis, University of Auckland, Auckland, New Zealand.

Bell, S. (2001). Landscape pattern, perception and visualisation in the visual management of forests. Landscape \& Urban Planning, 54(1), 201-211.

Bellard, C., Leclerc, C., Leroy, B., Bakkenes, M., Veloz, S., Thuiller, W., \& Courchamp, F. (2014). Vulnerability of biodiversity hotspots to global change. Global Ecology \& Biogeography, 23(12), 1376-1386.

Beninde, J., Veith, M., \& Hochkirch, A. (2015). Biodiversity in cities needs space: a meta-analysis of factors determining intra-urban biodiversity variation. Ecology letters, 18(6), 581-592.

Bennett, A. F. (1999). Linkages in the landscape: the role of corridors and connectivity in wildlife conservation. Gland and Cambridge: IUCN.

Berry, P. (2009). Biodiversity in the Balance: mitigation and adaptation conflicts and synergies. Sofia: Pensoft Publishers.

Biernacki, P., \& Waldorf, D. (1981). Snowball sampling: Problems and techniques of chain referral sampling. Sociological Methods \& Research, 10(2), 141-163.

Blackburn, T. M., Petchey, O. L., Cassey, P., \& Gaston, K. J. (2005). Functional diversity of mammalian predators and extinction in island birds. Ecology, 86(11), 2916-2923.

Boersma, P. D. (2008). Penguins as marine sentinels. BioScience, 58(7), 597-607.

Bonnington, C., Gaston, K. J., \& Evans, K. L. (2014). Squirrels in suburbia: influence of urbanisation on the occurrence and distribution of a common exotic mammal. Urban Ecosystems, 17(2), 533-546.

Borkin, K. M., \& Parsons, S. (2011). Home range and habitat selection by a threatened bat in exotic plantation forest. Forest Ecology \& Management, 262, 845-852.

Botequilha Leitão, A. \& Ahern, J. (2002) Applying landscape ecological concepts and metrics in sustainable landscape planning. Landscape \& Urban Planning, 59(2), 65-93.

Botequilha Leitão, A., Miller, J., Ahern, J. \& McGarigal, K. (2006). Measuring landscapes: A planner's handbook. Washington: Island Press.

Bowen, G. A. (2008). Naturalistic inquiry and the saturation concept: a research note. Qualitative Research, 8(1), 137-152.

Boyd, M. J., Bufill, M. C., \& Knee, R. M. (1993). Pervious and impervious runoff in urban catchments. Hydrological Sciences Journal, 38(6), 463-478.

Braaker, S., Kormann, U., Bontadina, F., \& Obrist, M. K. (2017). Prediction of genetic connectivity in urban ecosystems by combining detailed movement data, genetic data and multi-path modelling. Landscape \& Urban Planning, 160, $107-114$.

Brannstrom, C., \& Oliveira, A. M. (2000). Human modification of stream valleys in the western plateau of Sao Paulo, Brazil: implications for environmental narratives and management. Land Degradation \& Development, 11(6), 535-548.

Bräuniger, C., Knapp, S., Kühn, I., \& Klotz, S. (2010). Testing taxonomic and landscape surrogates for biodiversity in an urban setting. Landscape \& Urban Planning, 97(4), 283-295.

Breuste, J., Niemela, J. \& Snep, R. P. H. (2008) Applying landscape ecological principles in urban environments. Landscape Ecology, 23(10), 1139-1142.

Bridgewater, P. B. (1988). Biodiversity and landscape. Earth-Science Reviews, 25(5), 485-491.

Briggs, C. L. (1986). Learning how to ask: A sociolinguistic appraisal of the role of the interview in social science research (Vol. 1). Cambridge: Cambridge University Press. 
Brockerhoff, E. G., Jactel, H., Parrotta, J. A., Quine, C. P., \& Sayer, J. (2008). Plantation forests and biodiversity: Oxymoron or opportunity? Biodiversity \& Conservation, 17, 925-951.

Brockie, B. (2007). Native plants and animals-overview-Species unique to New Zealand. Te Ara - the Encyclopedia of New Zealand, Accessed on October 26, 2016 at: http://www.TeAra.govt.nz/en/native-plants-and-animals-overview/page-1

Broecker, W. (1975). Climatic change: are we on the brink of a pronounced global warming? Science, 189, 460-463.

Broitman, B. R., Szathmary, P. L., Mislan, K. A. S., Blanchette, C. A., \& Helmuth, B. (2009). Predator-prey interactions under climate change: the importance of habitat vs body temperature. Oikos, 118(2), 219-224.

Brook, B. W., Sodhi, N. S., \& Bradshaw, C. J. (2008). Synergies among extinction drivers under global change. Trends in Ecology \& Evolution, 23(8), 453-460.

Brown, K. P. (1997). Predation at nests of two New Zealand endemic passerines; implications for bird community restoration. Pacific Conservation Biology, 3(2), 91-98.

Bruns, D., Ortacesme, V., Stiles, R., de Vries, J., Holden, R., \& Jorgensen, K. (2010). Tuning Landscape Architecture Education in Europe. Vs, 26, 21-09.

Bryman, A. (2015). Social research methods. Oxford: Oxford University Press.

Buller, W.L. (1888). A history of the birds of New Zealand. London: Published by the author.

Bunin, J. S., \& Jamieson, I. G. (1996). Responses to a model predator of New Zealand's endangered takahe and its closest relative, the pukeko. Conservation Biology, 10(5), 1463-1466.

Burns, B., Innes, J., \& Day, T. (2012). The use and potential of pest-proof fencing for ecosystem restoration and fauna conservation in New Zealand. In: Somers, M. J., \& Hayward, M., (Eds.), Fencing for Conservation: Restriction of Evolutionary Potential or a Riposte to Threatening Processes?, (pp. 65-90), New York: Springer.

Burrows, L., Cieraad, E., \& Head, N. (2015). Scotch broom facilitates indigenous tree and shrub germination and establishment in dryland New Zealand. New Zealand Journal of Ecology, 39(1), 61-70.

Cai, W., Borlace, S., Lengaigne, M., Van Rensch, P., Collins, M., Vecchi, G.,... \& England, M. H. (2014). Increasing frequency of extreme El Niño events due to greenhouse warming. Nature Climate Change, 4(2), 111-116.

Calder, J. L., Cumming, G. S., Maciejewski, K., \& Oschadleus, H. D. (2015). Urban land use does not limit weaver bird movements between wetlands in Cape Town, South Africa. Biological Conservation, 187, 230-239.

Calthorpe, P. (2010). Urbanism in the age of climate change. Washington: Island Press.

Cameron, R. W., Blanuša, T., Taylor, J. E., Salisbury, A., Halstead, A. J., Henricot, B., \& Thompson, K. (2012). The domestic garden-Its contribution to urban green infrastructure. Urban Forestry \& Urban Greening, 11(2), 129-137.

Campbell, K. L (2006). A study of home ranges, movements, diet and habitat use of kererū (Hemiphaganovaeseelandiae) in the southeastern sector of Banks Peninsula, New Zealand. MSc Thesis, Lincoln University, Canterbury, New Zealand.

Carbó-Ramírez, P., \& Zuria, I. (2011). The value of small urban greenspaces for birds in a Mexican city. Landscape \& Urban Planning, 100(3), 213-222.

Cardille, J. A., \& Turner, M. G. (2017). Regional and Continental-Scale Perspectives on Landscape Pattern. In: Gergel, S. E., \& Turner, M. G., (Eds.), Learning Landscape Ecology, (pp. 157-173), New York: Springer.

Cardinale, B. J., Srivastava, D. S., Duffy, J. E., Wright, J. P., Downing, A. L., Sankaran, M., \& Jouseau, C. (2006). Effects of biodiversity on the functioning of trophic groups and ecosystems. Nature, 443(7114), 989-992.

Carrus, G., Scopelliti, M., Lafortezza, R., Colangelo, G., Ferrini, F., Salbitano, F., ... \& Sanesi, G. (2015). Go greener, feel better? The positive effects of biodiversity on the well-being of individuals visiting urban and peri-urban green areas. Landscape \& Urban Planning, 134, 221-228. 
Castillo-Montoya, M. (2016). Preparing for interview research: The interview protocol refinement framework. The Qualitative Report, 21(5), 811-831.

Castro, I., Brunton, D. H., Mason, K. M., Ebert, B., \& Griffiths, R. (2003). Life history traits and food supplementation affect productivity in a translocated population of the endangered Hihi (Stitchbird, Notiomystis cincta). Biological Conservation, 114(2), 271-280.

Catterall, C. P., Kanowski, J., Wardell-Johnson, G., Proctor, H. \& Reis, T. (2004). Quantifying the biodiversity values of reforestation: design issues and outcomes in Australian forest landscapes. In: Lunney, D., (Ed.), Conservation of Australia’s Forest Fauna, (pp 359-393), Royal Zoological Society of New South Wales, Mosman.

Chamaillé-Jammes, S., Fritz, H., \&Murindagomo, F. (2007). Detecting climate changes of concern in highly variable environments: Quantile regressions reveal that droughts worsen in Hwange National Park, Zimbabwe. Journal of Arid Environments, 71(3), 321-326.

Chambers, L. E. (2004). Delayed breeding in Little Penguins-evidence of climate change?. Australian Meteorological Magazine, 53(1), 13-19.

Chambers, L. E., Altwegg, R., Barbraud, C., Barnard, P., Beaumont, L. J., Crawford, R. J., ... \& Morellato, P. C. (2013). Phenological changes in the southern hemisphere. PloS one, 8(10), e75514.

Chambers, L. E., Barnard, P., Poloczanska, E. S., Hobday, A. J., Keatley, M. R., Allsopp, N., \& Underhill, L. G. (2017). Southern Hemisphere biodiversity and global change: Data gaps and strategies. Austral Ecology, 42(1), 20-30.

Chang, L. F., \& Huang, S. L. (2015). Assessing urban flooding vulnerability with an emergy approach. Landscape \& Urban Planning, 143, 11-24.

Chapple, D. G. (2016). Synthesising Our Current Knowledge of New Zealand Lizards. In: Chapple D. G., (Ed.), New Zealand Lizards, (pp. 1-11), Cham: Springer.

Charles, K. E., \& Linklater, W. L. (2015). The role of environmental engagement in tolerating urban bird problems. Human Dimensions of Wildlife, 20(2), 99-111.

Chauvenet, A. L., Ewen, J. G., Armstrong, D., \& Pettorelli, N. (2013). Saving the hihi under climate change: a case for assisted colonization. Journal of Applied Ecology, 50(6), 1330-1340.

Chazdon, R. L., \&Uriarte, M. (2016). Natural regeneration in the context of large-scale forest and landscape restoration in the tropics. Biotropica, 48(6), 709-715.

Chong, K. Y., Teo, S., Kurukulasuriya, B., Chung, Y. F., Rajathurai, S., \& Tan, H. T. W. (2014). Not all green is as good: Different effects of the natural and cultivated components of urban vegetation on bird and butterfly diversity. Biological Conservation, 171, 299-309.

Christie, J. E. (2014). Adapting to a changing climate: A proposed framework for the conservation of terrestrial native biodiversity in New Zealand. Wellington, Department of Conservation.

CIESIN - Center for International Earth Science Information Network (2004). Global Rural-Urban Mapping Project (GRUMP), Alpha Version: Urban Extents. Accessed at: http://sedac.ciesin.columbia.edu/gpw

Civantos, E., Thuiller, W., Maiorano, L., Guisan, A., \& Araújo, M. B. (2012). Potential impacts of climate change on ecosystem services in Europe: the case of pest control by vertebrates. BioScience, 62(7), 658-666.

Clarkson, B. D., \& Kirby, C. L. (2016). Ecological restoration in urban environments in New Zealand. Ecological Management \& Restoration, 17(3), 180-190.

Clarkson, B. D., Wehi, P. M., \& Brabyn, L. K. (2007). A spatial analysis of indigenous cover patterns and implications for ecological restoration in urban centres, New Zealand. Urban Ecosystems, 10(4), 441-457. 
Clout, M. N. (1990). The kereru and its forests. Birds International, 2(4), 10-19.

Clout, M. N., \& Hay, J. R. (1989). The importance of birds as browsers, pollinators and seed dispersers in New Zealand forests. New Zealand Journal of Ecology, 12, 27-33.

Clout, M., \& Gaze, P. D. (1984). Effects of plantation forestry on birds in New Zealand. The Journal of Applied Ecology, 21, 795-815.

Colding, J., Elmqvist, T., Lundberg, J., Ahrne' , K., Andersson, E., Barthel, S., Borgstro“ m, S., Duit, A., Ernstsson, H., Tengo“ , M., (2003). The Stockholm Urban Assessment (SUASweden). Stockholm: Millennium Ecosystem Assessment Sub-Global Summary Report.

Coleman, W. (1971). Biology in the nineteenth century: problems of form, function and transformation (Vol. 1). Cambridge: Cambridge University Press.

Colley, K., Brown, C., \& Montarzino, A. (2016). Restorative wildscapes at work: An investigation of the wellbeing benefits of greenspace at urban fringe business sites using 'go-along’ interviews. Landscape Research, 41(6), 1-18.

Collier, K. J., Parkyn, S. M., \& Rabeni, C. F. (1997). Koura: a keystone species. Water and Atmosphere, 5(1), 18-20.

Collinge, S. K. (1996). Ecological consequences of habitat fragmentation: implications for landscape architecture and planning. Landscape \& Urban Planning, 36(1), 59-77.

Collins, C. J., Rawlence, N. J., Prost, S., Anderson, C. N., Knapp, M., Scofield, R. P., ... \& Waters, J. M. (2014). Extinction and recolonization of coastal megafauna following human arrival in New Zealand. Proceedings of the Royal Society of London B: Biological Sciences, 281(1786), 20140097.

Common, M. S., \& Norton, T. W. (1992). Biodiversity: its conservation in Australia. Ambio, 21(3), 258-265.

Cook, E. A. (2002). Landscape structure indices for assessing urban ecological networks. Landscape \& Urban Planning, 58(2), 269-280.

Cooney, R. (2004). The Precautionary Principle in Biodiversity Conservation and Natural Resource Management: An issues paper for policy-makers, researchers and practitioners (No. 2). IUCN.

Corlett, R. T. (2005). Interactions between birds, fruit bats and exotic plants in urban Hong Kong, South China. Urban Ecosystems, 8, 275-283.

Cornelis, J., \& Hermy, M. (2004). Biodiversity relationships in urban and suburban parks in Flanders. Landscape \& Urban Planning, 69(4), 385-401.

Corry, R. C., \& Nassauer, J. I. (2005). Limitations of using landscape pattern indices to evaluate the ecological consequences of alternative plans and designs. Landscape and Urban Planning, 72(4), 265-280.

Cote, S., Durand, O., LaRoche, E., Warden, R., \& Sanctuary, Z. W. (2013). Evaluating the interactions between Wellington residents and the threatened kaka parrot. Prepared for Zealandia Wildlife Sanctuary. Worcester: Worcester Polytechnic Institute.

Cowan, P. E., Chilvers, B. L., Efford, M. G., \& McElrea, G. J. (1997). Effects of possum browsing on northern rata, Orongorongo Valley, Wellington, New Zealand. Journal of the Royal Society of New Zealand, 27(2), 173-179.

Cowling, R. M., Macdonald, I. A. W., \& Simmons, M. T. (1996). The Cape Peninsula, South Africa: physiographical, biological and historical background to an extraordinary hot-spot of biodiversity. Biodiversity \& Conservation, 5(5), 527-550.

Craig, J. L., Stewart, A. M., \& Douglas, M. E. (1981). The foraging of New Zealand honeyeaters. New Zealand Journal of Zoology, 8(1), 87-91. 
Craig, J., Anderson, S., Clout, M., Creese, B., Mitchell, N., Ogden, J., ... \& Ussher, G. (2000). Conservation issues in New Zealand. Annual Review of Ecology, Evolution \& Systematics, 31, 61-78.

Cushman, S. A., \& McGarigal, K. (2008). Landscape metrics, scales of resolution. In: Gadow, K. V., \& Pukkala, T., (Eds.), Designing Green Landscapes, (pp. 33-51), Dordrecht: Springer.

Cushman, S. A., McGarigal, K., \& Neel, M. C. (2008). Parsimony in landscape metrics: strength, universality, and consistency. Ecological Indicators, 8(5), 691-703.

Daehler, C. C. (2003). Performance comparisons of co-occurring native and alien invasive plants: implications for conservation and restoration. Annual Review of Ecology, Evolution, and Systematics, 34(1), 183-211.

Dale, V., H., Brown, S., Haeuber, R., A., Hobbs, N., T., Huntly, N., Naiman, R., J., .. \& Valone, T., J. (2000) Ecological principles and guidelines for managing the use of land. Ecological Applications, 10(3), 639-670.

Davies-Colley, R. J., Payne, G. W., \& Van Elswijk, M. (2000). Microclimate gradients across a forest edge. New Zealand Journal of Ecology, 24, 111-121.

Davis, A. (1994). Breeding biology of the New Zealand shore plover Thinornis novaeseelandiae. Notornis, 41, 195-208.

Davis, C. M., \& Meurk, C. D (2001). Protecting and restoring our natural heritage: A practical guide. Wellington, Department of Conservation.

Davis, R. A., Gole, C., \& Roberts, J. D. (2013). Impacts of urbanisation on the native avifauna of Perth, Western Australia. Urban Ecosystems, 16(3), 427-452.

Dawson, B. (2010). A History of Gardening in New Zealand: An Illustrated Social History of Gardening in New Zealand from Pre-European Times. Auckland, Random House New Zealand.

Dawson, J., \& Lucas, R. (2000). Nature guide to the New Zealand forest. Auckland: Godwit Press.

De Lange, P. J., Heenan, P. B., Norton, D. A., Rolfe, J. R., \& Sawyer, J. (2010). Threatened plants of New Zealand (p. 471). Christchurch, Canterbury University Press.

de Toledo, M. C. B., Donatelli, R. J., \& Batista, G. T. (2012). Relation between green spaces and bird community structure in an urban area in Southeast Brazil. Urban Ecosystems, 15(1), 111-131.

Dekrout, A. S., Clarkson, B. D., \& Parsons, S. (2014). Temporal and spatial distribution and habitat associations of an urban population of New Zealand long-tailed bats (Chalinolobus tuberculatus). New Zealand Journal of Zoology, 41(4), 285295.

Deming, M. E., \& Swaffield, S. (2011). Landscape architecture research: Inquiry, strategy, design. Hoboken, New Jersey, John Wiley and Sons.

Denyer, K., Burns, B., \& Ogden, J. (2006). Buffering of native forest edge microclimate by adjoining tree plantations. Austral Ecology, 31, 478-489.

Department of Conservation (2015). Department of Conservation biodiversity indicators: 2015 assessment - supplementary material. Wellington: Department of Conservation.

Department of Conservation (2016) Statement of Intent 2016-2020. Wellington: Department of Conservation.

Diamond, J. M., Ashmole, N. P., \& Purves, P. E. (1989). The present, past and future of human-caused extinctions [and discussion]. Philosophical Transactions of the Royal Society B: Biological Sciences, 325(1228), 469-477.

DiCicco-Bloom, B., \& Crabtree, B. F. (2006). The qualitative research interview. Medical Education, 40(4), 314-321.

Dickman, A. J. (2010). Complexities of conflict: the importance of considering social factors for effectively resolving human-wildlife conflict. Animal conservation, 13(5), 458-466. 
Dijak, W. D., \& Thompson III, F. R. (2000). Landscape and edge effects on the distribution of mammalian predators in Missouri. The Journal of Wildlife Management, 64(1), 209-216.

Dilks, P. E. T. E. R. (2004). Population status, breeding and ecology of Chatham Island Tui (Prosthemadera novaeseelandiae chathamensis). Notornis, 51(4), 217-226.

Dobrowski, S. Z. (2011). A climatic basis for microrefugia: the influence of terrain on climate. Global Change Biology, 17(2), 1022-1035.

Doick, K. J., Peace, A., \& Hutchings, T. R. (2014). The role of one large greenspace in mitigating London's nocturnal urban heat island. Science of the Total Environment, 493, 662-671.

Doody, B. J., Sullivan, J. J., Meurk, C. D., Stewart, G. H., \& Perkins, H. C. (2010). Urban realities: the contribution of residential gardens to the conservation of urban forest remnants. Biodiversity \& Conservation, 19(5), 1385-1400.

Dramstad, W. E., Olson, J. D. \& Forman, R. T. T. (1996) Landscape ecology principles in landscape architecture and landuse planning. Cambridge: Harvard University Graduate School of Design and Island Press.

Duffy, J. E. (2008). Why biodiversity is important to the functioning of real-world ecosystems. Frontiers in Ecology and the Environment, 7(8), 437-444.

Duffy, J. E., Cardinale, B. J., France, K. E., McIntyre, P. B., Thébault, E., \& Loreau, M. (2007). The functional role of biodiversity in ecosystems: incorporating trophic complexity. Ecology Letters, 10(6), 522-538.

Dugord, P. A., Lauf, S., Schuster, C., \& Kleinschmit, B. (2014). Land use patterns, temperature distribution, and potential heat stress risk-The case study Berlin, Germany. Computers, Environment \& Urban Systems, 48, 86-98.

Duncan, R. P., \& Blackburn, T. M. (2004). Extinction and endemism in the New Zealand avifauna. Global Ecology \& Biogeography, 13(6), 509-517.

Duncan, R. P., \& Young, J. R. (2000). Determinants of plant extinction and rarity 145 years after European settlement of Auckland, New Zealand Journal of Ecology, 81(11), 3048-3061.

Dungan, R. J., O'Cain, M. J., Lopez, M. L., \& Norton, D. A. (2002). Contribution by possums to seed rain and subsequent seed germination in successional vegetation, Canterbury, New Zealand. New Zealand Journal of Ecology, 26(2), 121127.

Dyball, R., Ives, C. D., \& White, I. (2013). Regional assessment of Oceania. In: Parnell, S., Schewenius, M., Sendstad, M., Seto, K. C., \& Wilkinson, C., (Eds.), Urbanization, Biodiversity and Ecosystem Services: Challenges and Opportunities, (pp. 287-289), Dordrecht; Heidelberg; New York; London: Springer.

Dymond J. R., Shepherd J. D., Newsome P.F., Belliss S. (2017) Estimating change in areas of indigenous vegetation cover in New Zealand from the New Zealand Land Cover Database (LCDB). New Zealand Journal of Ecology, 41(1):56-64.

Dymond, J. R., \& Shepherd, J. D. (2004). The spatial distribution of indigenous forest and its composition in the Wellington region, New Zealand, from ETM+ satellite imagery. Remote Sensing of Environment, 90, 116-125.

Eldredge, N. (2002). Life on Earth: AG (Vol. 1). Santa Barbara: ABC-CLIO.

Elmqvist, T., Fragkias, M., Goodness, J., Guneralp, B., Marcotullio, P.J., McDonald, R.I., Parnell, S., Schewenius, M., Sendstad, M., Seto, K.C., \& Wilkinson, C. (2013). Global Urbanisation, Biodiversity and Ecosystem Services: Challenges and Opportunities. Dordrecht: Springer.

Elton, C.S. (1958) The ecology of invasions by animals and plants. London: Methuenn.

Emeny, M. T., Powlesland, R. G., Henderson, I. M., \& Fordham, R. A. (2009). Feeding ecology of kererū (Hemiphaga novaeseelandiae) in podocarp-hardwood forest, Whirinaki Forest Park, New Zealand. New Zealand Journal of Ecology, 33(2), 114-124. 
Emmanuel, R., Loconsole, A. (2015). Green infrastructure as an adaptation approach to tackling urban overheating in the Glasgow Clyde Valley Region, UK. Landscape \& Urban Planning, 138: 71-86.

Empson, R. A. E. W. Y. N., \& Fastier, D. E. N. I. S. E. (2013). Translocations of North Island tomtits (Petroicamacrocephalatoitoi) and North Island robins (P. longipes) to Zealandia-Karori Sanctuary, an urban sanctuary. What have we learned. Notornis, 60(1), 63-69.

Evans, K. L., Newson, S. E., \& Gaston, K. J. (2009). Habitat influences on urban avian assemblages. Ibis, 151(1), 19-39.

Ewel, J. J., \&Putz, F. E. (2004). A place for alien species in ecosystem restoration. Frontiers in Ecology \& the Environment, 2(7), 354-360.

Ewen, J. G., Bensch, S., Blackburn, T. M., Bonneaud, C., Brown, R., Cassey, P., ... \& Pérez-Tris, J. (2012). Establishment of exotic parasites: the origins and characteristics of an avian malaria community in an isolated island avifauna. Ecology Letters, 15(10), 1112-1119.

Ewers, R. M., Kliskey, A. D., Walker, S., Rutledge, D., Harding, J. S., \& Didham, R. K. (2006). Past and future trajectories of forest loss in New Zealand. Biological Conservation, 133, 312-325.

Farina, A. (2006). Principles and methods in landscape ecology. Toward a science of landscape. Dordrecht: Springer.

Farinha-Marques, P., Lameiras, J. M., Fernandes, C., Silva, S., \& Guilherme, F. (2011). Urban biodiversity: a review of current concepts and contributions to multidisciplinary approaches. Innovation: European Journal of Social Science Research, 24(3), 247-271.

Fenner, M., \& Thompson, K. (2005). The ecology of seeds. Cambridge: Cambridge University Press.

Ferenc, M., Sedláček, O., \& Fuchs, R. (2014). How to improve urban greenspace for woodland birds: site and local-scale determinants of bird species richness. Urban Ecosystems, 17(2), 625-640.

Fernández, F. J., Alvarez-Vázquez, L. J., García-Chan, N., Martínez, A., \& Vázquez-Méndez, M. E. (2015). Optimal location of green zones in metropolitan areas to control the urban heat island. Journal of Computational and Applied Mathematics, 289, 412-425.

Fernandez, I. C., \& Morales, N. S. (2015). Prioritization of sites for plant species restoration in the Chilean Biodiversity Hotspot: A spatial multi-criteria decision analysis approach. BioRxiv, 026716.

Fernández-juricic, E. (2001). Avian spatial segregation at edges and interiors of urban parks in Madrid, Spain. Biodiversity \& Conservation, 10(8), 1303-1316.

Fernandez-Juricic, E., \& Jokimäki, J. (2001). A habitat island approach to conserving birds in urban landscapes: case studies from southern and northern Europe. Biodiversity \& Conservation, 10(12), 2023-2043.

Feyisa, G. L., Dons, K., \& Meilby, H. (2014). Efficiency of parks in mitigating urban heat island effect: An example from Addis Ababa. Landscape \& Urban Planning, 123, 87-95.

Fischer, J., Lindenmayer, D. B., \& Hobbs, R. (2009). Landscape pattern and biodiversity. In: Levin, S. A., Carpenter, S. R., Godfray, H. C. J., Kinzig, A. P., Loreau, M., Losos, J. B., ... Wilcove, D. S., (Eds.), The Princeton guide to ecology, (pp. 431-437), Princeton, New Jersey: Princeton University Press.

Fleming, C. A. (1975). The geological history of New Zealand and its biota. In: Kuschel, G., (Ed.), Biogeography and ecology in New Zealand, (pp. 1-86), Dordrecht: Springer.

Flemming, S.A. (2013). Little penguin. In Miskelly, C. M., (Ed.), New Zealand Birds Online. Accessed at: https://nzbirdsonline.org.nz

Flick, U. (1998). An introduction to qualitative research. London: Sage. 
Fontana, S., Sattler, T., Bontadina, F., \& Moretti, M. (2011). How to manage the urban green to improve bird diversity and community structure. Landscape \& Urban Planning, 101(3), 278-285.

Fontana, V., Radtke, A., Fedrigotti, V. B., Tappeiner, U., Tasser, E., Zerbe, S., \& Buchholz, T. (2013). Comparing land-use alternatives: Using the ecosystem services concept to define a multi-criteria decision analysis. Ecological Economics, 93, 128-136.

Forman, R. T. T. (1995) Land mosaics: The ecology of landscapes and regions. Cambridge, Cambridge University Press.

Forman, R. T. T. (2014) Urban ecology: science of cities. Cambridge: Cambridge University Press.

Forman, R. T. T., \& Collinge, S. K. (1997). Nature conserved in changing landscapes with and without spatial planning. Landscape \& Urban Planning, 37(1), 129-135.

Forman, R. T. T., \& Godron, M. (1981). Patches and structural components for a landscape ecology. BioScience, 31(10), 733-740.

Forman, R. T. T., \& Godron, M. (1986). Landscape ecology. New York, John Wiley \& Sons.

Foster, T. (2008). Plant heritage New Zealand: te whakapapa o nga rakua: interpreting the species features of native plants. Raupo.

Frank, S., \& Walz, U. (2017). Landscape metrics. In: Burkhard B., \& Maes J., (Eds.) Mapping Ecosystem Services, (pp. 8186), Sofia: Pensoft Publishers.

Frazier, T. G., Wood, N., Yarnal, B., \& Bauer, D. H. (2010). Influence of potential sea level rise on societal vulnerability to hurricane storm-surge hazards, Sarasota County, Florida. Applied Geography, 30(4), 490-505.

Freeman, C. (2005). Planning for urban nature in New Zealand. In: Dawson, M., (Ed.), Greening the city: bringing biodiversity back into the urban environment, (pp. 21-24), Christchurch, Royal New Zealand Institute of Horticulture.

Freeman, C., \& Buck, O. (2003). Development of an ecological mapping methodology for urban areas in New Zealand. Landscape \& Urban Planning, 63(3), 161-173.

Gabites, I. (1993). Wellington's living cloak. Wellington: Wellington Botanical Society \& Victoria University Press.

Gallo, T., Fidino, M., Lehrer, E. W., \& Magle, S. B. (2017). Mammal diversity and metacommunity dynamics in urban green spaces: implications for urban wildlife conservation. Ecological Applications, 27(8), 2330-2341.

Garcia, D., Zamora, R., \&Amico, G. C. (2010). Birds as suppliers of seed dispersal in temperate ecosystems: conservation guidelines from real-world landscapes. Conservation Biology, 24(4), 1070-1079.

Garden, J. G., McAlpine, C. A., \& Possingham, H. P. (2010). Multi-scaled habitat considerations for conserving urban biodiversity: native reptiles and small mammals in Brisbane, Australia. Landscape Ecology, 25(7), 1013-1028.

Garibaldi, A., \& Turner, N. (2004). Cultural keystone species: implications for ecological conservation and restoration. Ecology \& Society, 9(3), 1.

Gill, S. E., Handley, J. F., Ennos, A. R., \& Pauleit, S. (2007). Adapting cities for climate change: the role of the green infrastructure. Built Environment, 33(1), 115-133.

Gillies, C., \& Clout, M. (2003). The prey of domestic cats (Feliscatus) in two suburbs of Auckland City, New Zealand. Journal of Zoology, 259(3), 309-315.

Gillner, S., Vogt, J., Tharang, A., Dettmann, S., \& Roloff, A. (2015). Role of street trees in mitigating effects of heat and drought at highly sealed urban sites. Landscape and Urban Planning, 143, 33-42.

Gitay, H., Suarez, A., \& Watson, R. T. (2002). Climate Change and Biodiversity, IPCC Technical Paper V-April 2002.

Given D, Meurk C. (2000). Biodiversity of the urban environment: the importance of indigenous species and the role urban environments can play in their preservation. In: Stewart GH, Ignatieva M. E., (Eds). In: Urban biodiversity and ecology 
as a basis for holistic planning and design: proceedings of a workshop held at Lincoln University, 28-29 October 2000, Christchurch: Wickliffe Press, p. 22-33.

Goldson, S. L., Bourdôt, G. W., Brockerhoff, E. G., Byrom, A. E., Clout, M. N., McGlone, M. S., ... \& Templeton, M. D. (2015). New Zealand pest management: current and future challenges. Journal of the Royal Society of New Zealand, 45(1), 31-58.

Goodman, L. A. (1961). Snowball sampling. Annals of Mathematical Statistics, 32(1), 148-170.

Government Printer (1887). Results of a Census of the Colony of New Zealand 1886. Wellington, Government Printer.

Gray, E. R., \& van Heezik, Y. (2016). Exotic trees can sustain native birds in urban woodlands. Urban Ecosystems, 19(1), 315-329.

Grayson, K. L., Mitchell, N. J., Monks, J. M., Keall, S. N., Wilson, J. N., \& Nelson, N. J. (2014). Sex ratio bias and extinction risk in an isolated population of tuatara (Sphenodon punctatus). PloS one, 9(4), e94214.

Grimm, N. B., Faeth, S. H., Golubiewski, N. E., Redman, C. L., Wu, J., Bai, X., \& Briggs, J. M. (2008). Global change and the ecology of cities. Science, 319(5864), 756-760.

Groat, L. N., \& Wang, D. (2002). Architectural Research Methods. New Jersey: John Wiley \& Sons.

Groffman, P. M., Cavender-Bares, J., Bettez, N. D., Grove, J. M., Hall, S. J., Heffernan, J. B., ... \& Nelson, K. (2014). Ecological homogenization of urban USA. Frontiers in Ecology and the Environment, 12(1), 74-81.

Gubrium, J. F., \& Holstein, J. A. (2002). Handbook of interview research: Context and method. London: Sage.

Guest, G., Bunce, A., \& Johnson, L. (2006). How many interviews are enough? An experiment with data saturation and variability. Field Methods, 18(1), 59-82.

Gustafson, E. J. (1998). Quantifying landscape spatial pattern: what is the state of the art?. Ecosystems, 1(2), 143-156.

Haddad, N. (2009) Principles of reserve design. In: Levin, S.A., (Ed.), The Princeton Guide to Ecology, Princeton University Press, Princeton, New Jersey, pp. 529-537.

Hagerman, S., Dowlatabadi, H., Satterfield, T., \& McDaniels, T. (2010). Expert views on biodiversity conservation in an era of climate change. Global Environmental Change, 20(1), 192-207.

Hahs, A. K., McDonnell, M. J., McCarthy, M. A., Vesk, P. A., Corlett, R. T., Norton, B. A., ... \& Williams, N. S. (2009). A global synthesis of plant extinction rates in urban areas. Ecology Letters, 12(11), 1165-1173.

Halpin, P. N. (1997). Global climate change and natural-area protection: management responses and research directions. Ecological Applications, 7(3), 828-843.

Hämäläinen, S., Fey, K., \& Selonen, V. (2018). Habitat and nest use during natal dispersal of the urban red squirrel (Sciurus vulgaris). Landscape \& Urban Planning, 169, 269-275.

Hansen, H. S. (2010). Modelling the future coastal zone urban development as implied by the IPCC SRES and assessing the impact from sea level rise. Landscape \& Urban Planning, 98(3), 141-149.

Harper, M. J. (2006). Home range and den use of common brushtail possums (Trichosurus vulpecula) in urban forest remnants. Wildlife Research, 32(8), 681-687.

Harris, J. A., Hobbs, R. J., Higgs, E., \& Aronson, J. (2006). Ecological restoration and global climate change. Restoration Ecology, 14(2), 170-176.

Harvell, C. D., Mitchell, C. E., Ward, J. R., Altizer, S., Dobson, A. P., Ostfeld, R. S., \& Samuel, M. D. (2002). Climate warming and disease risks for terrestrial and marine biota. Science, 296(5576), 2158-2162.

Hawksworth, D. L. (1995). Biodiversity: measurement and estimation. London: Chapman \& Hall.

Heather, B. D., \& Robertson, H. A. (2005). The Field Guide to the Birds of New Zealand. Auckland: Penguin New Zealand. 
Hector, A., \& Bagchi, R. (2007). Biodiversity and ecosystem multifunctionality. Nature, 448(7150), 188-190.

Heller, N. E., \& Zavaleta, E. S. (2009). Biodiversity management in the face of climate change: a review of 22 years of recommendations. Biological Conservation, 142(1), 14-32.

Hill, M. J., Braaten, R., Veitch, S. M., Lees, B. G., \& Sharma, S. (2005). Multi-criteria decision analysis in spatial decision support: the ASSESS analytic hierarchy process and the role of quantitative methods and spatially explicit analysis. Environmental Modelling \& Software, 20(7), 955-976.

Hoffmann, A. A., \& Sgrò, C. M. (2011). Climate change and evolutionary adaptation. Nature, 470(7335), 479-485.

Holdaway, R. N. (1989). New Zealand's pre-human avifauna and its vulnerability. New Zealand Journal of Ecology, 12, 1125.

Holdaway, R. N. (1999). Introduced predators and avifaunal extinction in New Zealand. In: MacPhee, R. D. E., (Ed.), Extinctions in near time, (pp. 189-238), New York: Springer.

Honnay, O., Piessens, K., Van Landuyt, W., Hermy, M., \& Gulinck, H. (2003). Satellite based land use and landscape complexity indices as predictors for regional plant species diversity. Landscape \& Urban Planning, 63(4), 241-250.

Hooper, D. U., Adair, E. C., Cardinale, B. J., Byrnes, J. E., Hungate, B. A., Matulich, K. L., ... \& O’Connor, M. I. (2012). A global synthesis reveals biodiversity loss as a major driver of ecosystem change. Nature, 486(7401), 105-108.

Howe, L., Castro, I. C., Schoener, E. R., Hunter, S., Barraclough, R. K., \& Alley, M. R. (2012). Malaria parasites (Plasmodium spp.) infecting introduced, native and endemic New Zealand birds. Parasitology research, 110(2), 913-923.

Huang, I. B., Keisler, J., \&Linkov, I. (2011). Multi-criteria decision analysis in environmental sciences: ten years of applications and trends. Science of the Total Environment, 409(19), 3578-3594.

Hulse, D., Branscomb, A., Enright, C., Johnson, B., Evers, C., Bolte, J., \& Ager, A. (2016). Anticipating surprise: Using agent-based alternative futures simulation modeling to identify and map surprising fires in the Willamette Valley, Oregon USA. Landscape \& Urban Planning, 156, 26-43.

Hulse, D., Eilers, J., Freemark, K., Hummon, C., \& White, D. (2000). Planning alternative future landscapes in Oregon: evaluating effects on water quality and biodiversity. Landscape Journal, 19(1-2), 1-19.

Hurlimann, A., Barnett, J., Fincher, R., Osbaldiston, N., Mortreux, C., \& Graham, S. (2014). Urban planning and sustainable adaptation to sea-level rise. Landscape \& Urban Planning, 126, 84-93.

Hutchison, M. A. S. (2009). Interactions between habitat fragmentation and invasions: factors driving exotic plant invasions in native forest remnants, West Coast, New Zealand. Doctoral Thesis, University of Canterbury, Christchurch, New Zealand.

Ignatieva, M., Meurk, C., \& Stewart, G. (2008). Low impact urban design and development (LIUDD): matching urban design and urban ecology. Landscape review, 12(2), 61-73.

Ignatieva, M., Stewart, G. H., \& Meurk, C. (2011). Planning and design of ecological networks in urban areas. Landscape \& Ecological Engineering, 7(1), 17-25.

Ikin, K., Knight, E., Lindenmayer, D. B., Fischer, J., \& Manning, A. D. (2013). The influence of native versus exotic streetscape vegetation on the spatial distribution of birds in suburbs and reserves. Diversity \& Distributions, 19(3), 294306.

Imai, H., \& Nakashizuka, T. (2010). Environmental factors affecting the composition and diversity of avian community in mid-to late breeding season in urban parks and green spaces. Landscape \& Urban Planning, 96(3), 183-194.

Innes J, Kelly D, Overton JM, Gillies C (2010) Predation and other factors currently limiting New Zealand forest birds. New Zealand Journal of Ecology 34, 86-114. 
Innes, J., Burns, B., Sanders, A., \& Hayward, M. W. (2015 a). The impact of private sanctuary networks on reintroduction programs. In: Armstrong, D., P., Hayward, M., W., Moro, D., Seddon, P., J., (Eds.), Advances in Reintroduction Biology of Australian and New Zealand Fauna, (pp. 185-200), Victoria: Commonwealth Scientific and Industrial Research Organization Publishing, Victoria, Australia.

Innes, J., Kelly, D., Overton, J. M., \& Gillies, C. (2010). Predation and other factors currently limiting New Zealand forest birds. New Zealand Journal of Ecology, 34(1), 86-114.

Innes, J., King, C., Bartlam, S., Forrester, G., \& Howitt, R. (2015 b). Predator control improves nesting success in Waikato forest fragments. New Zealand Journal of Ecology, 39(2), 245-253.

Innes, J., Lee, W. G., Burns, B., Campbell-Hunt, C., Watts, C., Phipps, H., \& Stephens, T. (2012). Role of predator-proof fences in restoring New Zealand's biodiversity: a response to Scofield et al. (2011). New Zealand Journal of Ecology, 36(2), 232-238.

IPCC (2007). Intergovernmental panel on climate change. Climate change 2007: Synthesis report.

IPCC (1995). Scientific Assessments of Climate Change. The Policymaker's Summary of Working Group 1 to the Intergovernmental Panel on Climate Change, WMO/UNEP.

IPCC (2014). Summary for Policymakers. In: Climate Change 2014: Mitigation of Climate Change. Contribution of Working Group III to the Fifth Assessment Report of the Intergovernmental Panel on Climate Change [Edenhofer, O., R. Pichs-Madruga, Y. Sokona, E. Farahani, S. Kadner, K. Seyboth, A. Adler, I. Baum, S. Brunner, P. Eickemeier, B. Kriemann, J. Savolainen, S. Schlömer, C. von Stechow, T. Zwickel and J.C. Minx (Eds.)]. Cambridge; New York: Cambridge University Press.

Isbell, F., Calcagno, V., Hector, A., Connolly, J., Harpole, W. S., Reich, P. B., ... \& Weigelt, A. (2011). High plant diversity is needed to maintain ecosystem services. Nature, 477(7363), 199-202.

Israel, G. D. (1992). Determining sample size. University of Florida Cooperative Extension Service, Institute of Food and Agriculture Sciences, EDIS.

Ives, C. D., Lentini, P. E., Threlfall, C. G., Ikin, K., Shanahan, D. F., Garrard, G. E., ... \& Rowe, R. (2016). Cities are hotspots for threatened species. Global Ecology \& Biogeography, 25(1), 117-126.

Jacobs, S., Burkhard, B., Van Daele, T., Staes, J., \& Schneiders, A. (2015). ‘The Matrix Reloaded’: A review of expert knowledge use for mapping ecosystem services. Ecological Modelling, 295, 21-30.

Jarvie S, Recio MR, Adolph SC, Seddon PJ, Cree A. (2016). Resource selection by tuatara following translocation: a comparison of wild-caught and captive-reared juveniles. New Zealand Journal of Ecology, 40, 334-341.

Jasmani, Z., Ravn, H. P., \& van den Bosch, C. C. K. (2017). The influence of small urban parks characteristics on bird diversity: A case study of Petaling Jaya, Malaysia. Urban Ecosystems, 20(1), 227-243.

Jo, H. K. (2002). Impacts of urban greenspace on offsetting carbon emissions for middle Korea. Journal of Environmental Management, 64(2), 115-126.

Jo, H. K., \& McPherson, G. E. (1995). Carbon storage and flux in urban residential greenspace. Journal of Environmental Management, 45(2), 109-133.

Jokimäki, J., Selonen, V., Lehikoinen, A., \& Kaisanlahti-Jokimäki, M. L. (2017). The role of urban habitats in the abundance of red squirrels (Sciurus vulgaris, L.) in Finland. Urban Forestry \& Urban Greening, 27, 100-108.

Jones, C., Moss, K., \& Sanders, M. (2005). Diet of hedgehogs (Erinaceus europaeus) in the upper Waitaki Basin, New Zealand: implications for conservation. New Zealand Journal of Ecology, 29(1), 29-35. 
Jones, D. N. \& Reynolds, S. J. (2008). Feeding birds in our towns and cities: a global research opportunity. Journal of Avian Biology, 39(3), 265-271.

Jones, G. W., \& Douglass, M. (2008). Mega-urban regions in Pacific Asia: Urban dynamics in a global era. Singapore: NUS Press.

Jongman, R. H. G. (2002) Homogenisation and fragmentation of the European landscapes: Ecological consequences and solutions. Landscape \& Urban Planning, 58(2-4), 211-221.

Joppa, L. N., O'Connor, B., Visconti, P., Smith, C., Geldmann, J., Hoffmann, M., ... \& Ahmed, S. E. (2016). Filling in biodiversity threat gaps. Science, 352(6284), 416-418.

Jupiter, S., Mangubhai, S., \& Kingsford, R. T. (2014). Conservation of biodiversity in the Pacific Islands of Oceania: challenges and opportunities. Pacific Conservation Biology, 20(2), 206-220.

Kang, W., Minor, E. S., Park, C. R., \& Lee, D. (2015). Effects of habitat structure, human disturbance, and habitat connectivity on urban forest bird communities. Urban Ecosystems, 18(3), 857-870.

Kaspersen, P. S., Høegh Ravn, N., Arnbjerg-Nielsen, K., Madsen, H., \& Drews, M. (2015). Influence of urban land cover changes and climate change for the exposure of European cities to flooding during high-intensity precipitation. Proceedings of the International Association of Hydrological Sciences (IAHS), 370, 21-27.

Kelly, D. (1994). The evolutionary ecology of mast seeding. Trends in Ecology \& Evolution, 9(12), 465-470.

Kelly, D., Ladley, J. J., Robertson, A. W., Anderson, S. H., Wotton, D. M., \& Wiser, S. K. (2010). Mutualisms with the wreckage of an avifauna: the status of bird pollination and fruit-dispersal in New Zealand. New Zealand Journal of Ecology, 34(1), 66-85.

Kennedy, C. (2003). Conservation thresholds for land use planners. Washington DC: Environmental Law Institute.

Khera, N., Mehta, V., \& Sabata, B. C. (2009). Interrelationship of birds and habitat features in urban greenspaces in Delhi, India. Urban Forestry \& Urban Greening, 8(3), 187-196.

Kikillus, K. H., Chambers, G. K., Farnworth, M. J., \& Hare, K. M. (2017). Research challenges and conservation implications for urban cat management in New Zealand. Pacific Conservation Biology, 23(1), 15-24.

King, C. M. (1983). The relationships between beech (Nothofagus sp.) seedfall and populations of mice (Mus musculus), and the demographic and dietary responses of stoats (Mustela erminea), in three New Zealand forests. Journal of Animal Ecology, 52(1), 141-166.

King, C. M. (2017). Liberation and spread of stoats (Mustela erminea) and weasels (M. nivalis) in New Zealand, 18831920. New Zealand Journal of Ecology, 41(2), 163-177

King, C. M., Hay, J. R., Smale, M. C., Leathwick, J. R., \& Beveridge, A. E. (2015). Forests and Native Wildlife. In: King, C., M., Gaukrodger, D. J., \& Ritchie, N., A., (Eds.), The Drama of Conservation, (pp. 19-42), Cham: Springer.

Kingsford, R. T., Watson, J. E., Lundquist, C. J., Venter, O., Hughes, L., Johnston, E. L., ... \& Morley, C. (2009). Major conservation policy issues for biodiversity in Oceania. Conservation Biology, 23(4), 834-840.

Kong, F., Yin, H., Wang, C., Cavan, G., \& James, P. (2014). A satellite image-based analysis of factors contributing to the green-space cool island intensity on a city scale. Urban Forestry \& Urban Greening, 13(4), 846-853.

Kühn, I., Brandl, R., \& Klotz, S. (2004). The flora of German cities is naturally species rich. Evolutionary Ecology Research, 6(5), 749-764.

Kunz, T. H., Braun de Torrez, E., Bauer, D., Lobova, T., \& Fleming, T. H. (2011). Ecosystem services provided by bats. Annals of the New York Academy of Sciences, 1223(1), 1-38.

Kupfer, J. A. (2006) National assessments of forest fragmentation in the US. Global Environmental Change, 16(1), 73-82. 
Kupfer, J. A. (2012). Landscape ecology and biogeography: rethinking landscape metrics in a post-FRAGSTATS landscape. Progress in Physical Geography, 36(3), 400-420.

Lambin, E. F., Turner, B. L., Geist, H. J., Agbola, S. B., Angelsen, A., Bruce, J. W., ... \& George, P. (2001). The causes of land-use and land-cover change: moving beyond the myths. Global Environmental Change, 11(4), 261-269.

Landcare Research (2012). Potential Vegetation of New Zealand. Data reproduced with the permission of Landcare Research New Zealand Limited. Accessed at: https://ris.scinfo.org.nz/layer/289-potential-vegetation-of-new-zealand/

Landcare Research (2010). New Zealand Digital Elevation Model North Island 25 metre. Data reproduced with the permission of Landcare Research New Zealand Limited. Accessed at: https://ris.scinfo.org.nz/layer/131-nzdem-northisland-25-metre

Landcare Research (2015). LCDB v4.1 - Land Cover Database version 4.1, Mainland New Zealand. Accessed at: https://ris.scinfo.org.nz/layer/423-lcdb-v41-land-cover-database-version-41-mainland-new-zealand

Langemeyer, J., Gómez-Baggethun, E., Haase, D., Scheuer, S., \&Elmqvist, T. (2016). Bridging the gap between ecosystem service assessments and land-use planning through Multi-Criteria Decision Analysis (MCDA). Environmental Science \& Policy, 62, 45-56.

Latham, A. D. M., Latham, M. C., Anderson, D. P., Cruz, J., Herries, D., \& Hebblewhite, M. (2015). The GPS craze: six questions to address before deciding to deploy GPS technology on wildlife. New Zealand Journal of Ecology, 39(1), 143-152.

Leathwick, J., McGlone, M. S., Walker, S., \& Briggs, C. (2004). New Zealand’s potential vegetation pattern. Whenua, Lincoln: Landcare Research.

Lefcheck, J. S., \& Duffy, J. E. (2015). Multitrophic functional diversity predicts ecosystem functioning in experimental assemblages of estuarine consumers. Ecology, 96(11), 2973-2983.

Lepczyk, C. A., La Sorte, F. A., Aronson, M. F., Goddard, M. A., MacGregor-Fors, I., Nilon, C. H., \& Warren, P. S. (2017). Global Patterns and Drivers of Urban Bird Diversity. In: Murgui E., Hedblom M., (Eds.), Ecology and Conservation of Birds in Urban Environments, (pp. 13-33), Cham: Springer.

Leveau, L. M., \& Leveau, C. M. (2016). Does urbanization affect the seasonal dynamics of bird communities in urban parks?. Urban Ecosystems, 19(2), 631-647.

Lin, T.P., Tsai, K.T., Hwang, R.L., Matzarakis, A. (2012). Quantification of the effect of thermal indices and sky view factor on park attendance. Landscape and Urban Planing. 107, 137-146.

Liu, H., \& Piao, S. (2013). Drought threatened semi-arid ecosystems in the Inner Asia. Agricultural and Forest Meteorology, 178-179: 1-2.

Liu, Z., Wimberly, M. C., Lamsal, A., Sohl, T. L., \& Hawbaker, T. J. (2015). Climate change and wildfire risk in an expanding wildland-urban interface: a case study from the Colorado Front Range Corridor. Landscape Ecology, 30(10), 1943-1957.

Lizée, M. H., Manel, S., Mauffrey, J. F., Tatoni, T., \& Deschamps-Cottin, M. (2012). Matrix configuration and patch isolation influences override the species-area relationship for urban butterfly communities. Landscape Ecology, 27(2), 159-169.

Lloyd, D. G. (1985). Progress in understanding the natural history of New Zealand plants. New Zealand Journal of Botany, 23(4), 707-722. 
Loehman, R. A., Keane, R. E., Holsinger, L. M., \& Wu, Z. (2017). Interactions of landscape disturbances and climate change dictate ecological pattern and process: spatial modeling of wildfire, insect, and disease dynamics under future climates. Landscape Ecology, 32(7), 1447-1459.

Logan, K. E., Brunsell, N. A., Jones, A. R., \& Feddema, J. J. (2010). Assessing spatiotemporal variability of drought in the US central plains. Journal of Arid Environments, 74(2), 247-255.

Luo, Z., \& Asproudi, C. (2015). Subsurface urban heat island and its effects on horizontal ground-source heat pump potential under climate change. Applied Thermal Engineering, 90, 530-537.

Lyver, P. O. B., Akins, A., Phipps, H., Kahui, V., Towns, D. R., \& Moller, H. (2015). Key biocultural values to guide restoration action and planning in New Zealand. Restoration Ecology, 24(3), 314-323.

MacArthur, R., \& Wilson, E. (1967). The theory of biogeography. New Jersey: Princeton University Press.

MacCarter, L. E., \& Gaynor, D. L. (1980). Gorse: A subject for biological control in New Zealand. New Zealand Journal of Experimental Agriculture, 8(3-4), 321-330.

MacLeod, C. J., Tinkler, G., Gormley, A. M., \& Spurr, E. B. (2012). Measuring occupancy for an iconic bird species in urban parks. New Zealand Journal of Ecology, 36(3), 1-10.

MacLeod, L. J., Dickson, R., Leckie, C., Stephenson, B. M., \& Glen, A. S. (2015). Possum control and bird recovery in an urban landscape, New Zealand. Conserv. Evidence, 12, 44-47.

Madden, F. (2004). Creating coexistence between humans and wildlife: global perspectives on local efforts to address human-wildlife conflict. Human Dimensions of Wildlife, 9(4), 247-257.

Maimaitiyiming, M., Ghulam, A., Tiyip, T., Pla, F., Latorre-Carmona, P., Halik, Ü., ... \& Caetano, M. (2014). Effects of green space spatial pattern on land surface temperature: Implications for sustainable urban planning and climate change adaptation. ISPRS Journal of Photogrammetry \& Remote Sensing, 89, 59-66.

Makan, T., Castro, I., Robertson, A. W., Joy, M. K., \& Low, M. (2014). Habitat complexity and management intensity positively influence fledging success in the endangered hihi (Notiomystis cincta). New Zealand Journal of Ecology, 38(1), 53-63.

Malcolm, J. R., Liu, C., Neilson, R. P., Hansen, L., \& Hannah, L. E. E. (2006). Global warming and extinctions of endemic species from biodiversity hotspots. Conservation Biology, 20(2), 538-548.

Mander, C. J., Hay, J. R., \& Powlesland, R. (1998). Monitoring and management of kererū (Hemiphaganovaeseelandiae) (p. 44). Wellington: New Zealand Department of Conservation.

Manfredo, M. J., \& Dayer, A. A. (2004). Concepts for exploring the social aspects of human-wildlife conflict in a global context. Human Dimensions of Wildlife, 9(4), 1-20.

Mantyka-pringle, C. S., Martin, T. G., \& Rhodes, J. R. (2012). Interactions between climate and habitat loss effects on biodiversity: a systematic review and meta-analysis. Global Change Biology, 18(4), 1239-1252.

Mariano, V., \&Christianini, A. V. (2016). Reproductive phenology, seed removal and early regeneration in relation to distance from parental plants of a native palm in small Atlantic forest fragments. Acta Botanica Brasilica, 30(2), 176182.

Mark, A. F. (2010). Threatened Plants of New Zealand. New Zealand Journal of Botany, 48, 250-252.

Markham, A., Dudley, N., \& Stolton, S. (1993). Some like it hot: climate change, biodiversity and the survival of species. Gland: WWF-International.

Martin, T. G., Kuhnert, P. M., Mengersen, K., \& Possingham, H. P. (2005). The power of expert opinion in ecological models using Bayesian methods: impact of grazing on birds. Ecological Applications, 15(1), 266-280. 
Maslin, M. 2004. Global Warming, a very short introduction. Oxford: Oxford University Press.

Mayer-Pinto, M., Johnston, E. L., Hutchings, P. A., Marzinelli, E. M., Ahyong, S. T., Birch, G., ... \&Gribben, P. E. (2015). Sydney Harbour: a review of anthropogenic impacts on the biodiversity and ecosystem function of one of the world's largest natural harbours. Marine \& Freshwater Research, 66(12), 1088-1105.

McAlpine, K. G., Lamoureaux, S. L., Timmins, S. M., \& Wotton, D. M. (2017). Native woody plant recruitment in lowland forests invaded by non-native ground cover weeds and mammals. New Zealand Journal of Ecology, 41(1), 65.

McCleery, R. A., Moorman, C., \& Peterson, M., N. (2014). Urban Wildlife Conservation: Theory and Practice. New York: Springer.

McClure, C. J., Korte, A. C., Heath, J. A., \& Barber, J. R. (2015). Pavement and riparian forest shape the bird community along an urban river corridor. Global Ecology \& Conservation, 4, 291-310.

McCracken, I. J. (1993). Natural Succession as a Management Strategy for Gorse and Broom Covered Land: Minimum Interference Management. Christchurch: Canterbury Regional Council.

McDonald, R. I., Kareiva, P., \& Forman, R. T. (2008). The implications of current and future urbanization for global protected areas and biodiversity conservation. Biological Conservation, 141(6), 1695-1703.

McDonnell, M. J., \& Hahs, A. K. (2015). Adaptation and adaptedness of organisms to urban environments. Annual Review of Ecology, Evolution, and Systematics, 46, 261-280.

McDonnell, M. J., \& Pickett, S. T. (1990). Ecosystem structure and function along urban-rural gradients: an unexploited opportunity for ecology. Ecology, 71(4), 1232-1237.

McGarigal K. (2015). Help. Accessed at: http://www.umass.edu/landeco/research/fragstats/documents/fragstats.help.4.2.pdf.

McGarigal, K., \& Marks. B. J. (1995). FRAGSTATS: spatial pattern analysis program for quantifying landscape structure. Portland: USDA Forest Service, Pacific Northwest Research Station.

McGarigal, K., \& Cushman, S. A. (2002). Comparative evaluation of experimental approaches to the study of habitat fragmentation effects. Ecological Applications, 12(2), 335-345.

McGarigal, K., Cushman, S. A., \& Ene, E. (2012). FRAGSTATS v4: Spatial Pattern Analysis Program for Categorical and Continuous Maps. Computer software program produced by the authors at the University of Massachusetts, Amherst. Accessed at: http://www.umass.edu/landeco/research/fragstats/fragstats.html

McGlone, M. S. (1983). Polynesian deforestation of New Zealand: a preliminary synthesis. Archaeology in Oceania, 18, 1125.

McGlone, M. S. (1989). The Polynesian settlement of New Zealand in relation to environmental and biotic changes. New Zealand Journal of Ecology, 12, 115-129.

McGlone, M. S., \& Walker, S. (2011). Potential effects of climate change on New Zealand's terrestrial biodiversity and policy recommendations for mitigation, adaptation and research. Science for Conservation 312. Wellington: Department of Conservation.

McHale, M. R., Hall, S. J., Majumdar, A., \& Grimm, N. B. (2017). Carbon lost and carbon gained: a study of vegetation and carbon trade-offs among diverse land uses in Phoenix, Arizona. Ecological Applications, 27(2), 644-661.

McKinney, M.L., (2002). Urbanization, biodiversity, and conservation. BioScience, 52(10), 883-890.

McLellan, R., Iyengar, L., Jeffries, B., \& Oerlemans, N. (2014). Living Planet Report 2014: species and spaces, people and places. Gland: World Wide Fund for Nature. 
McLennan, J. A., Potter, M. A., Robertson, H. A., Wake, G. C., Colbourne, R., Dew, L., ... \& Reid, J. (1996). Role of predation in the decline of kiwi, Apteryx spp., in New Zealand. New Zealand Journal of Ecology, 20(1), 27-35.

McWethy, D. B., Whitlock, C., Wilmshurst, J. M., McGlone, M. S., Fromont, M., Li, X., ... Cook, E. R. (2010). Rapid landscape transformation in South Island, New Zealand, following initial Polynesian settlement. Proceedings of the National Academy of Sciences, 107, 21343-21348.

Meijering, J. V., Tobi, H., van den Brink, A., Morris, F., \& Bruns, D. (2015). Exploring research priorities in landscape architecture: An international Delphi study. Landscape \& Urban Planning, 137, 85-94.

Melville, D. S., \& Battley, P. F. (2006). Shorebirds in New Zealand. The Stilt, 50, 295-303.

Menkhorst, P., \& Knight, F. (2001). A field guide to mammals of Australia. Melbourne: Oxford University Press.

Meurk, C. D. (1999). Proposed quantitative thresholds for adequate protection of natural areas at district scale. In: Norton, D., \& Roper-Lindsay, J., (Eds.), Criteria for assessing ecological significance under Section 6(c) of the Resource Management Act 1991, (Appendix). Wellington: Ministry for the Environment.

Meurk, C. D. (2005). Cities are cultural and ecological keys to biodiverse futures. In: Dawson, M., (Ed.), Greening the city: bringing biodiversity back into the urban environment, (pp. 301-310), Christchurch, Royal New Zealand Institute of Horticulture.

Meurk, C. D., \& Hall, G. M. (2006). Options for enhancing forest biodiversity across New Zealand's managed landscapes based on ecosystem modelling and spatial design. New Zealand Journal of Ecology, (30)1: 131-146.

Meurk, C. D., \& Swaffield, S. R. (2000). A landscape ecological framework for indigenous regeneration in rural New Zealand-Aotearoa. Landscape \& Urban Planning, 50(1), 129-144.

Meurk, C. D., \& Swaffield, S. R. (2007). Cities as complex landscapes: biodiversity opportunities, landscape configurations and design directions. New Zealand Garden Journal, 10(1), 10-19.

Meurk, C. D., Blaschke, P. M., \& Simcock, R. (2013). Ecosystem services in New Zealand cities. Ecosystem services in New Zealand: conditions and trends. Lincoln: Manaaki Whenua Press.

Meurk, C. D., Sullivan, J., \& McWilliam, W. (2016). Vegetation History and Dynamics in New Zealand: Future Scenarios and Improved Trajectories: Towards Restoring Natural Patterns. In: Box E., (Ed.), Vegetation Structure and Function at Multiple Spatial, Temporal and Conceptual Scales, (pp. 517-528), Cham: Springer.

Meyer, W. B., \& Turner, B. L. (1992). Human population growth and global land-use/cover change. Annual Review of Ecology \& Systematics, 23, 39-61.

Middelbeek, L., Kolle, K., \&Verrest, H. (2014). Built to last? Local climate change adaptation and governance in the Caribbean-The case of an informal urban settlement in Trinidad and Tobago. Urban Climate, 8, 138-154.

Milburn, L. A. S., \& Brown, R. D. (2003). The relationship between research and design in landscape architecture. Landscape and urban planning, 64(1-2), 47-66.

Millennium Ecosystem Assessment (2005). Current state and trends. Washington DC: World Resources Institute.

Mills, L. S., Soulé, M. E., \& Doak, D. F. (1993). The keystone-species concept in ecology and conservation. BioScience, 43(4), 219-224.

Ministry for Primary Industries, (2015). Look-up Tables for Forestry in the Emissions Trading Scheme. Wellington: New Zealand.

Ministry for the Environment (1997). The state of New Zealand's environment. Wellington: Ministry of the Environment. 
Ministry for the Environment, (2014). Climate change projections for the Wellington and Wairarapa region. Accessed at: http://www.mfe.govt.nz/climate-change/how-climate-change-affects-nz/how-might-climate-change-affect-myregion/wellington

Moller, H., Frampton, C., Hocken, A. G., McLean, I. G., Saffer, V. and Sheridan, L. (2000) The importance of seabird research for New Zealand, New Zealand Journal of Zoology, 27(4), 255-260.

Moorcroft, P. R. (2009). Biodiversity patterns in managed and natural landscapes. In: Levin, S.A., (Ed.), The Princeton Guide to Ecology, Princeton University Press, Princeton, New Jersey, pp. 445-457.

Mora, C., \& Sale, P. F. (2011). Ongoing global biodiversity loss and the need to move beyond protected areas: a review of the technical and practical shortcomings of protected areas on land and sea. Marine Ecology Progress Series, 434, 251266.

Morrison, M. L., Marcot, B., \& Mannan, W. (2012). Wildlife-habitat relationships: concepts and applications. Washington: Island Press.

Morton, A. G. (1981). History of Botanical Science. An account of the development of botany from ancient times to the present day. London: Academic Press.

Morton, S. R. (1990). The impact of European settlement on the vertebrate animals of arid Australia: a conceptual model. In Proceedings of the Ecological Society of Australia, 16, 201-213.

Müller, N., \& Kamada, M. (2011). URBIO: an introduction to the International Network in Urban Biodiversity and Design. Landscape \& Ecological Engineering, 7(1), 1-8.

Mulligan, G. F., \& Crampton, J. P. (2005). Population growth in the world’s largest cities. Cities, 22(5), 365-380.

Murcia, C. (1995). Edge effects in fragmented forests: implications for conservation. Trends in Ecology \& Evolution, 10(2), 58-62.

Murgui, E. (2009). Influence of urban landscape structure on bird fauna: a case study across seasons in the city of Valencia (Spain). Urban Ecosystems, 12(3), 249.

Murgui, E., \& Hedblom, M. (2017). Ecology and Conservation of Birds in Urban Environments. Cham: Springer.

Murphy, E. C., \& Dowding, J. E. (1994). Range and diet of stoats (Mustela erminea) in a New Zealand beech forest. New Zealand Journal of Ecology, 18(1), 11-18.

Murray, M. H., \& St Clair, C. C. (2017). Predictable features attract urban coyotes to residential yards. Journal of Wildlife Management, 81(4), 593-600.

Myeong, S., Nowak, D. J., \& Duggin, M. J. (2006). A temporal analysis of urban forest carbon storage using remote sensing. Remote Sensing of Environment, 101(2), 277-282.

Myers, J. H., \& Bazely, D. (2003). Ecology and control of introduced plants. Cambridge: Cambridge University Press.

Myers, N. (1993). Biodiversity and the precautionary principle. Ambio, 22(2-3), 74-79.

Myers, N., Mittermeier, R. A., Mittermeier, C. G., Da Fonseca, G. A., \& Kent, J. (2000). Biodiversity hotspots for conservation priorities. Nature, 403(6772), 853-858.

Myint, S. W., Zheng, B., Talen, E., Fan, C., Kaplan, S., Middel, A., ... \& Brazel, A. (2015). Does the spatial arrangement of urban landscape matter? Examples of urban warming and cooling in Phoenix and Las Vegas. Ecosystem Health \& Sustainability, 1(4), 1-15.

Nam, W. H., Hayes, M. J., Svoboda, M. D., Tadesse, T., \& Wilhite, D. A. (2015). Drought hazard assessment in the context of climate change for South Korea. Agricultural Water Management, 160, 106-117.

Nash, R. F. (1989). The rights of nature: a history of environmental ethics. Madison: University of Wisconsin Press. 
Nassauer, J. I., \& Corry, R. C. (2004). Using normative scenarios in landscape ecology. Landscape ecology, 19(4), 343-356.

Naveh, Z. (1994). Biodiversity and landscape management. In: Kim, K. C., Weaver, R. D., (Eds.), Biodiversity and Landscapes, (pp. 187-208), Cambridge: Cambridge University Press.

Neel, M. C., McGarigal, K., \& Cushman, S. A. (2004). Behavior of class-level landscape metrics across gradients of class aggregation and area. Landscape Ecology, 19(4), 435-455.

Nelson, N. J., Romijn, R. L., Dumont, T., Reardon, J. T., Monks, J. M., Hitchmough, R. A., ... \& Briskie, J. V. (2016). Lizard Conservation in Mainland Sanctuaries. In: Chapple, D. G., (Ed.), New Zealand lizards. New Zealand Lizards, (pp. 321-339), Cham: Springer.

Ng, E., Chen, L., Wang, Y., \& Yuan, C. (2012). A study on the cooling effects of greening in a high-density city: an experience from Hong Kong. Building \& Environment, 47, 256-271.

Niebuhr, C. N. (2016). Avian malaria transmission dynamics in New Zealand: investigating host and vector relationships along an elevational gradient, Doctoral Thesis: University of Otago, Dunedin, New Zealand.

Nielsen, A. B., van den Bosch, M., Maruthaveeran, S., \& van den Bosch, C. K. (2014). Species richness in urban parks and its drivers: a review of empirical evidence. Urban Ecosystems, 17(1), 305-327.

Niemelä, J. (1999). Ecology and urban planning. Biodiversity \& Conservation, 8, 119-131.

NIWA - National Institute for Water and Atmospheric Research (2001). Climate data and activities. Accessed at: https://www.niwa.co.nz/education-and-training/schools/resources/climate

Noble, I.R. \& Slatyer, R.O. (1980) The use of vital attributes to predict successional changes in plant communities subject to recurrent disturbances. Vegetatio, 43, 5-21.

Noreika, N., \& Kotze, D. J. (2012). Forest edge contrasts have a predictable effect on the spatial distribution of carabid beetles in urban forests. Journal of Insect Conservation, 16(6), 867-881.

Norton, B. A., Coutts, A. M., Livesley, S. J., Harris, R. J., Hunter, A. M., \& Williams, N. S. (2015). Planning for cooler cities: A framework to prioritise green infrastructure to mitigate high temperatures in urban landscapes. Landscape and Urban Planning, 134, 127-138.

Norton, D. A., Young, L. M., Byrom, A. E., Clarkson, B. D., Lyver, P. O. B., McGlone, M. S., \& Waipara, N. W. (2016). How do we restore New Zealand's biological heritage by 2050?. Ecological Management \& Restoration, 17(3), 170179.

Noss, R. F. (2001). Beyond Kyoto: forest management in a time of rapid climate change. Conservation Biology, 15(3), 578590.

Noss, R. F., \& Cooperrider, A. (1994). Saving nature's legacy: protecting and restoring biodiversity. Washington: Island Press.

Nowak, D. J., \& Crane, D. E. (2002). Carbon storage and sequestration by urban trees in the USA. Environmental Pollution, 116(3), 381-389.

Nowak, D. J., Greenfield, E. J., Hoehn, R. E., \& Lapoint, E. (2013). Carbon storage and sequestration by trees in urban and community areas of the United States. Environmental Pollution, 178, 229-236.

Noy, C. (2008). Sampling knowledge: The hermeneutics of snowball sampling in qualitative research. International Journal of Social Research Methodology, 11(4), 327-344.

Odum, E. P., Odum, H. T., \& Andrews, J. (1971). Fundamentals of ecology (Vol. 3). Philadelphia: Saunders.

Ogden, J., Braggins, J., Stretton, K., \& Anderson, S. (1997). Plant species richness under Pinus radiata stands on the central North Island volcanic plateau, New Zealand. New Zealand Journal of Ecology, 21, 17-29. 
Oliver, A. J., Hong-Wa, C., Devonshire, J., Olea, K. R., Rivas, G. F., \& Gahl, M. K. (2011). Avifauna richness enhanced in large, isolated urban parks. Landscape \& Urban Planning, 102(4), 215-225.

Olson, D. M., Dinerstein, E., Wikramanayake, E. D., Burgess, N. D., Powell, G. V., Underwood, E. C., ... \& Loucks, C. J. (2001). Terrestrial Ecoregions of the World: A New Map of Life on Earth A new global map of terrestrial ecoregions provides an innovative tool for conserving biodiversity. BioScience, 51(11), 933-938.

Olson, D., DellaSala, D. A., Noss, R. F., Strittholt, J. R., Kass, J., Koopman, M. E., \& Allnutt, T. F. (2012). Climate change refugia for biodiversity in the Klamath-Siskiyou ecoregion. Natural Areas Journal, 32(1), 65-74.

O'Neill, R. V., Milne, B. T., Turner, M. G., \& Gardner, R. H. (1988). Resource utilization scales and landscape pattern. Landscape Ecology, 2(1), 63-69.

Opdam, P., Steingröver, E., \& van Rooij, S. (2006). Ecological networks: A spatial concept for multi-actor planning of sustainable landscapes. Landscape \& Urban Planning, 75, 322-332.

Oppenheim, A. N. (2000). Questionnaire design, interviewing and attitude measurement. New York: Bloomsbury Publishing.

Ossola, A., \& Niemelä, J. (2017). Urban Biodiversity: From Research to Practice. Abingdon-on-Thames: Routledge.

Otago Regional Council. (n.d.). Gorse and broom factsheet. Accessed at: http://www.orc.govt.nz/Information-andServices/Pest-Control/Plant-pests/Gorse-and-broom

Our Natural Capital (2015). Wellington's biodiversity strategy and action plan 2015. Wellington: Wellington City Council.

Pacheco, R., \& Vasconcelos, H. L. (2007). Invertebrate conservation in urban areas: ants in the Brazilian Cerrado. Landscape \& Urban Planning, 81(3), 193-199.

Paker, Y., Yom-Tov, Y., Alon-Mozes, T., \& Barnea, A. (2014). The effect of plant richness and urban garden structure on bird species richness, diversity and community structure. Landscape \& Urban Planning, 122, 186-195.

Park, G. (1999). NgāUruora: Ecology and History in a New Zealand Landscape. Wellington: Victoria University Press.

Park, J. H., \& Cho, G. H. (2016). Examining the Association between Physical Characteristics of Green Space and Land Surface Temperature: A Case Study of Ulsan, Korea. Sustainability, 8(8), 777.

Parkes, J., \& Murphy, E. (2003). Management of introduced mammals in New Zealand. New Zealand Journal of Zoology, 30(4), 335-359.

Parmesan, C. (2006). Ecological and evolutionary responses to recent climate change. Annual Review of Ecology, Evolution \& Systematics, 37, 637-669.

Pasari, J. R., Levi, T., Zavaleta, E. S., \& Tilman, D. (2013). Several scales of biodiversity affect ecosystem multifunctionality. Proceedings of the National Academy of Sciences, 110(25), 10219-10222.

Paton, P. W. (1994). The effect of edge on avian nest success: how strong is the evidence?. Conservation Biology, 8(1), 1726.

Patrick, B. (1994). Hawkdun ecological District invertebrate survey. Wellington: Department of Conservation.

Pauchard, A., \& Barbosa, O. (2013). Regional assessment of Latin America: rapid urban development and social economic inequity threaten biodiversity hotspots. In: Parnell, S., Schewenius, M., Sendstad, M., Seto, K. C., \& Wilkinson, C., (Eds.), Urbanization, biodiversity and ecosystem services: Challenges and opportunities, (pp. 589-608), Dordrecht: Springer.

Pauchard, A., Aguayo, M., Peña, E., \&Urrutia, R. (2006). Multiple effects of urbanization on the biodiversity of developing countries: the case of a fast-growing metropolitan area (Concepción, Chile). Biological Conservation, 127(3), $272-281$. 
Pauleit, S., \& Duhme, F. (2000). Assessing the environmental performance of land cover types for urban planning. Landscape \& Urban Planning, 52(1), 1-20.

Pauleit, S., Liu, L., Ahern, J., \& Kazmierczak, A. (2011). Multifunctional green infrastructure planning to promote ecological services in the city. In: Niemela, J., Breuste, J. H., Guntenspergen, G. \& McIntyre, N. E. (Eds). Urban ecology: patterns, processes, and applications, (pp. 272-285), Oxford: Oxford University Press.

Pawson, E., \& Brooking, T. (2002). Environmental histories of New Zealand. Melbourne: Oxford University Press.

Pawson, S. M., Ecroyd, C. E., Seaton, R., Shaw, W. B., \& Brockerhoff, E. G. (2010). New Zealand's exotic plantation forests as habitats for threatened indigenous species. New Zealand Journal of Ecology, 34, 342-355.

Payton, I. J., Fenner, M., \& Lee, W. G. (2002). Keystone species: the concept and its relevance for conservation management in New Zealand. Wellington: Department of Conservation.

Pedersen Zari, M. (2012). Ecosystem services analysis for the design of regenerative urban built environments. Doctoral Thesis: Victoria University of Wellington, Wellington, New Zealand.

Pedersen Zari, M. (2015). Ecosystem services analysis: Mimicking ecosystem services for regenerative urban design. International Journal of Sustainable Built Environment, 4, 145-157.

Peel, M. C., Finlayson, B. L., \& McMahon, T. A. (2007). Updated world map of the Köppen-Geiger climate classification. Hydrology \& Earth System Sciences Discussions, 4(2), 439-473.

Pereira, H. M., Leadley, P. W., Proença, V., Alkemade, R., Scharlemann, J. P., Fernandez-Manjarrés, J. F., ... \& Chini, L. (2010). Scenarios for global biodiversity in the $21^{\text {st }}$ century. Science, 330(6010), 1496-1501.

Pernetta, J. L., Elder, R., \& D Humphrey, S. (1994). Impacts of climate change on ecosystems and species: Implications for protected areas. In 4. World Congress on National Parks and Protected AreasEne 1992Caracas (Venezuela) (No. 333.95I34). IUCN, Gland. Marine and Coastal Areas Programme.

Perry, G. L., Wheeler, A. B., Wood, J. R., \& Wilmshurst, J. M. (2014). A high-precision chronology for the rapid extinction of New Zealand moa (Aves, Dinornithiformes). Quaternary Science Reviews, 105, 126-135.

Peters, M. A., Hamilton, D., \& Eames, C. (2015). Action on the ground: A review of community environmental groups' restoration objectives, activities and partnerships in New Zealand. New Zealand Journal of Ecology, 39(2), 179-189.

Pickett, S. T., Cadenasso, M. L., \& McGrath, B. (2013). Resilience in ecology and urban design: linking theory and practice for sustainable cities. New York: Springer.

Pierce, R. J., Gardiner, C., Moodie, H., Robertson, H. A., \& Sporle, W. (2006). Sustainable Management of Brown Kiwi and other threatened birds in Northland (No. 1193). Wildlands Contract Report.

Pizo, M. A. (1997). Seed dispersal and predation in two populations of Cabraleacanjerana (Meliaceae) in the Atlantic Forest of southeastern Brazil. Journal of Tropical Ecology, 13(4), 559-577.

Platt, A., \& Lill, A. (2006). Composition and conservation value of bird assemblages of urban 'habitat islands': do pedestrian traffic and landscape variables exert an influence?. Urban Ecosystems, 9(2), 83-97.

Port Stephens Council. (2002). Port Stephens Council Comprehensive Koala Plan of Management (CKPoM) - June 2002. Port Stephens: Port Stephens Council with the Australian Koala Foundation.

Potter, J., \& Hepburn, A. (2012). Eight challenges for interview researchers. Handbook of Interview Research, London: Sage.

Powlesland, R. G., Moran, L. R., \& Wotton, D. M. (2011). Satellite tracking of kereru (Hemiphaga novaeseelandiae) in Southland, New Zealand: impacts, movements and home range. New Zealand Journal of Ecology, 35(3), 229-235. 
Pryde, M. A., O’Donnell, C. F., \& Barker, R. J. (2005). Factors influencing survival and long-term population viability of New Zealand long-tailed bats (Chalinolobus tuberculatus): implications for conservation. Biological Conservation, 126(2), 175-185.

Pulliam, H. R. (1988). Sources, sinks, and population regulation. American Naturalist, 132(5), 652-661.

Qiu, G. Y., LI, H. Y., Zhang, Q. T., Wan, C. H. E. N., Liang, X. J., \& Li, X. Z. (2013). Effects of evapotranspiration on mitigation of urban temperature by vegetation and urban agriculture. Journal of Integrative Agriculture, 12(8), 13071315.

Radeloff, V. C., Hammer, R. B., Stewart, S. I., Fried, J. S., Holcomb, S. S., \& McKeefry, J. F. (2005). The wildland-urban interface in the United States. Ecological Applications, 15(3), 799-805.

Rastandeh, A. (2015). Challenges and potentials in using alternative landscape futures during climate change: A literature review and survey study. Urbani Izziv, 26(2), 83-102.

Ray, S. (2013). Investigation into the halt in population recovery of the southern New Zealand dotterel. Postgraduate Report: University of Otago, Dunedin, New Zealand.

Rea, L. M., \& Parker, R. A. (2014). Designing and conducting survey research: A comprehensive guide. New Jersey: John Wiley \& Sons.

Reardon, J. T., Whitmore, N., Holmes, K. M., Judd, L. M., Hutcheon, A. D., Norbury, G., \& Mackenzie, D. I. (2012). Predator control allows critically endangered lizards to recover on mainland New Zealand. New Zealand Journal of Ecology, 36(2), 141-150.

Rebelo, A. G., Holmes, P. M., Dorse, C., \& Wood, J. (2011). Impacts of urbanization in a biodiversity hotspot: conservation challenges in Metropolitan Cape Town. South African Journal of Botany, 77(1), 20-35.

Recio, M. R., Mathieu, R., \& Seddon, P. J. (2011). Design of a GPS backpack to track European hedgehogs Erinaceus europaeus. European Journal of Wildlife Research, 57(6), 1175-1178.

Recio, M. R., Mathieu, R., Latham, M. C., Latham, A. D. M., \& Seddon, P. J. (2013). Quantifying fine-scale resource selection by introduced European hedgehogs (Erinaceus europaeus) in ecologically sensitive areas. Biological Invasions, 15(8), 1807-1818.

Recio, M. R., Mathieu, R., Virgós, E., \& Seddon, P. J. (2014). Quantifying fine-scale resource selection by introduced feral cats to complement management decision-making in ecologically sensitive areas. Biological Invasions, 16(9), 19151927.

Rega, C. C., Nilon, C. H., \& Warren, P. S. (2015). Avian abundance patterns in relation to the distribution of small urban greenspaces. Journal of Urban Planning and development, 141(3), A4015002.

Reside, A. E., Welbergen, J. A., Phillips, B. L., Wardell-Johnson, G. W., Keppel, G., Ferrier, S., ... \& VanDerWal, J. (2014). Characteristics of climate change refugia for Australian biodiversity. Austral Ecology, 39(8), 887-897.

Rhee, J., Park, S., \& Lu, Z. (2014). Relationship between land cover patterns and surface temperature in urban areas. GIScience \& Remote Sensing, 51(5), 521-536.

Ridley, A. R. (1998). The feeding ecology and habitat use of kereru and bellbird in a modified forest remnant, South Canterbury. Doctoral Thesis: Lincoln University, Lincoln, New Zealand.

Rio Declaration on Environment and Development, (1992). Un.Doc/CoNF.151/5/Rev.1.

Roaf, S., Crichton, D., \& Nicol, F. (2009). Adapting buildings and cities for climate change. Abingdon-on-Thames: Routledge. 
Robertson, H. A., \& Colbourne, R. M. (2004). Survival of little spotted kiwi (Apteryx owenii) on Kapiti Island. Notornis, 51(3), 161-162.

Robertson, H. A., Baird, K., Dowding, J. E., Elliott, G. P., Hitchmough, R. A., Miskelly, C. M., ... \& Taylor, G. A. (2017). Conservation status of New Zealand birds, 2016. New Zealand Threat Classification Series 19. Wellington: Department of Conservation.

Robinson, O. C. (2014). Sampling in interview-based qualitative research: A theoretical and practical guide. Qualitative Research in Psychology, 11(1), 25-41.

Röder, G., Baglione, V., Bolopo, D., Marcos, J. M., Trnka, A., \& Canestrari, D. (2016). Small Emissions with Major Consequences: Specialized Malodorous Defences in Birds. In: Schulte B., Goodwin T., Ferkin M., (Eds.), Chemical Signals in Vertebrates, (pp. 281-302), Cham: Springer.

Romagosa, F., Eagles, P. F., \& Lemieux, C. J. (2015). From the inside out to the outside in: Exploring the role of parks and protected areas as providers of human health and well-being. Journal of Outdoor Recreation \& Tourism, 10, 70-77.

Romijn, R., Adams, L., Hitchmough, R. (2012). Lizard strategy for the Wellington region 2012-20. Wellington: Wellington Regional Lizard Network.

Rorison, I. H., Sutton, F., \& Hunt, R. (1986). Local climate, topography and plant growth in Lathkill Dale NNR. I. A twelve-year summary of solar radiation and temperature. Plant, Cell \& Environment, 9(1), 49-56.

Rosenzweig, C., \& Neofotis, P. (2013). Detection and attribution of anthropogenic climate change impacts. Wiley Interdisciplinary Reviews: Climate Change, 4(2), 121-150.

Rosenzweig, C., Casassa, G., Karoly, D. J., Imeson, A., Liu, C., Menzel, A., ... \& Hanson, C. E. (2007). Assessment of observed changes and responses in natural and managed systems. Climate change, 79-131.

Ruffell, J., \& Didham, R. K. (2017). Conserving biodiversity in New Zealand's lowland landscapes: does forest cover or pest control have a greater effect on native birds?. New Zealand Journal of Ecology, 41(1), 23-33.

Russell, J. C., Innes, J. G., Brown, P. H., \&Byrom, A. E. (2015). Predator-free New Zealand: conservation country. BioScience, 65(5), 520-525.

Saarela, S. R., \& Rinne, J. (2016). Knowledge brokering and boundary work for ecosystem service indicators. An urban case study in Finland. Ecological Indicators, 61, 49-62.

Sagar, P. M., \& Scofield, R. P. (2006). Survival, density and population composition of bellbirds (Anthornis melanura) on the Poor Knights Islands, New Zealand. New Zealand Journal of Zoology, 33(4), 249-257.

Saito, N. (2014). Challenges for adapting Bangkok’s flood management systems to climate change. Urban Climate, 9, 89100.

Sandström, U. G., Angelstam, P., \& Mikusiński, G. (2006). Ecological diversity of birds in relation to the structure of urban green space. Landscape \& Urban Planning, 77(1), 39-53.

Sanz, V., \& Caula, S. (2015). Assessing bird assemblages along an urban gradient in a Caribbean island (Margarita, Venezuela). Urban Ecosystems, 18(3), 729-746.

Sattler, T., Duelli, P., Obrist, M. K., Arlettaz, R., \& Moretti, M. (2010). Response of arthropod species richness and functional groups to urban habitat structure and management. Landscape Ecology, 25(6), 941-954.

Saunders, A., \& Norton, D. A. (2001). Ecological restoration at mainland islands in New Zealand. Biological Conservation, 99(1), 109-119.

Savard, J. P. L., Clergeau, P., \& Mennechez, G. (2000). Biodiversity concepts and urban ecosystems. Landscape \& Urban Planning, 48(3), 131-142. 
Schindler, S., von Wehrden, H., Poirazidis, K., Wrbka, T., \& Kati, V. (2013). Multiscale performance of landscape metrics as indicators of species richness of plants, insects and vertebrates. Ecological Indicators, 31, 41-48.

Schmitz, O. J., \& Barton, B. T. (2014). Climate change effects on behavioral and physiological ecology of predator-prey interactions: implications for conservation biological control. Biological Control, 75, 87-96.

Schoener, E. R., Banda, M., Howe, L., Castro, I. C., \& Alley, M. R. (2014). Avian malaria in New Zealand. New Zealand Veterinary Journal, 62(4), 189-198.

Schulz, J. J., \&Schröder, B. (2017). Identifying suitable multifunctional restoration areas for Forest Landscape Restoration in Central Chile. Ecosphere, 8(1).

Schwarz, N., Moretti, M., Bugalho, M. N., Davies, Z. G., Haase, D., Hack, J., ... \& Knapp, S. (2017). Understanding biodiversity-ecosystem service relationships in urban areas: A comprehensive literature review. Ecosystem services, 27, 161-171.

Schwarz, N., Schlink, U., Franck, U., \& Großmann, K. (2012). Relationship of land surface and air temperatures and its implications for quantifying urban heat island indicators - an application for the city of Leipzig (Germany). Ecological Indicators, 18, 693-704.

Seale, C. (1999). Quality in qualitative research. Qualitative inquiry, 5(4), 465-478.

Setälä, H. M., Francini, G., Allen, J. A., Hui, N., Jumpponen, A., \& Kotze, D. J. (2016). Vegetation Type and Age Drive Changes in Soil Properties, Nitrogen, and Carbon Sequestration in Urban Parks under Cold Climate. Frontiers in Ecology \& Evolution, 4, 93.

Seto, K. C., \& Fragkias, M. (2005). Quantifying spatiotemporal patterns of urban land-use change in four cities of China with time series landscape metrics. Landscape Ecology, 20(7), 871-888.

Shaffer, M. L. (1981). Minimum population sizes for species conservation. Biological Sciences, 31(2), 131-134.

Shaw, R. C., \& MacKinlay, R. D. (2016). Destruction of a North Island robin (Petroicalongipes) nest by a little spotted kiwi (Apteryx owenii). Notornis, 63, 46-49.

Shepherd, W., \& Cook, W. (1988). The Botanic Garden Wellington: a New Zealand history 1840-1987. Wellington: Millwood Press.

Sheppard, C. S., Burns, B., \& Stanley, M. (2016). Future-proofing weed management for the effects of climate change: is New Zealand underestimating the risk of increased plant invasions?. New Zealand Journal of Ecology, 40(3), 398-405.

Shwartz, A., Shirley, S., \& Kark, S. (2008). How do habitat variability and management regime shape the spatial heterogeneity of birds within a large Mediterranean urban park?. Landscape \& Urban Planning, 84(3), 219-229.

Silverman, D. (2004) Qualitative research: theory, method and practice. London: Sage.

Sing, K. W., Jusoh, W. F., Hashim, N. R., \& Wilson, J. J. (2016). Urban parks: refuges for tropical butterflies in Southeast Asia?. Urban Ecosystems, 19(3), 1131-1147.

Singh, V. S., Pandey, D. N., \& Chaudhry, P. (2010). Urban forests and open green spaces: lessons for Jaipur, Rajasthan India. Jaipur: Rajasthan State Pollution Control Board.

Smith, L. S., Broyles, M. E., Larzleer, H. K., \& Fellowes, M. D. (2015). Adding ecological value to the urban lawnscape. Insect abundance and diversity in grass-free lawns. Biodiversity and conservation, 24(1), 47-62.

Smith, P. G. (2007). Characteristics of urban natural areas influencing winter bird use in southern Ontario, Canada. Environmental Management, 39(3), 338-352. 
Snep, R. P., Kooijmans, J. L., Kwak, R. G., Foppen, R. P., Parsons, H., Awasthy, M., ... \& van Heezik, Y. M. (2016). Urban bird conservation: presenting stakeholder-specific arguments for the development of bird-friendly cities. Urban Ecosystems, 19(4), 1535-1550.

Soule, M.E. (1991). Land use planning and wildlife maintenance: guidelines for conserving wildlife in an urban landscape. Journal of the American Planning Association, 57(3): 313-323.

Soulsbury, C. D., \& White, P. C. (2016). Human-wildlife interactions in urban areas: a review of conflicts, benefits and opportunities. Wildlife Research, 42(7), 541-553.

Southon, G. E., Jorgensen, A., Dunnett, N., Hoyle, H., \& Evans, K. L. (2018). Perceived species-richness in urban green spaces: Cues, accuracy and well-being impacts. Landscape and Urban Planning, 172, 1-10.

Spellerberg, I. F., \& Given, D. R. (2008). Trees in urban and city environments: A review of the selection criteria with particular reference to nature conservation in New Zealand cities. Landscape Review, 12, 19-31.

Spurr, E. B. (2012). New Zealand Garden Bird Survey-analysis of the first four years. New Zealand Journal of Ecology, 36(3), 1-13.

Spurr, E. B., Rod, S., \& Tranter, K. P. (2011). Food preferences of the bellbird (Anthornis melanura) in native forest remnants on the Port Hills, Banks Peninsula, New Zealand. Notornis, 58(3-4), 139-157.

Stanbury, M., \& Briskie, J. V. (2015). I smell a rat: Can New Zealand birds recognize the odor of an invasive mammalian predator?. Current Zoology, 61(1), 34-41.

Standish, R. J., Hobbs, R. J., \& Miller, J. R. (2013). Improving city life: options for ecological restoration in urban landscapes and how these might influence interactions between people and nature. Landscape Ecology, 28(6), 12131221.

Starling-Windhof, A., Massaro, M., \& Briskie, J. V. (2011). Differential effects of exotic predator-control on nest success of native and introduced birds in New Zealand. Biological Invasions, 13(4), 1021-1028.

Statistics New Zealand (2002). Monitoring progress towards a sustainable New Zealand. Wellington: Statistics New Zealand.

Sternberg, M., Gabay, O., Angel, D., Barneah, O., Gafny, S., Gasith, A., ... \& Rilov, G. (2015). Impacts of climate change on biodiversity in Israel: an expert assessment approach. Regional Environmental Change, 15(5), 895-906.

Stevens, H. (2006). Native birds in forest remnants. Doctoral Thesis: University of Auckland, Auckland, New Zealand.

Stevenson, C. D., Ferryman, M., Nevin, O. T., Ramsey, A. D., Bailey, S., \& Watts, K. (2013). Using GPS telemetry to validate least-cost modeling of gray squirrel (Sciurus carolinensis) movement within a fragmented landscape. Ecology \& Evolution, 3(7), 2350-2361.

Stewart, A. M., \& Craig, J. L. (1985). Movements, status, access to nectar, and spatial organisation of the tui. New Zealand Journal of Zoology, 12(4), 664-666.

Stewart, G. H., Ignatieva, M. E., Meurk, C. D., \& Earl, R. D. (2004). The re-emergence of indigenous forest in an urban environment, Christchurch, New Zealand. Urban Forestry \& Urban Greening, 2(3), 149-158.

Stewart, G. H., Meurk, C. D., Ignatieva, M. E., Buckley, H. L., Magueur, A., Case, B. S., ... Parker, M.(2009). URban Biotopes of Aotearoa New Zealand (URBANZ) II: Floristics, biodiversity and conservation values of urban residential and public woodlands, Christchurch. Urban Forestry \& Urban Greening, 8, 149-162.

Storch, I., Woitke, E., \& Krieger, S. (2005). Landscape-scale edge effect in predation risk in forest-farmland mosaics of central Europe. Landscape Ecology, 20(8), 927-940.

Stork, N. E. (1988). Insect diversity: facts, fiction and speculation. Biological journal of the Linnean Society, 35(4), 321-337. 
Strohbach, M. W., Lerman, S. B., \& Warren, P. S. (2013). Are small greening areas enhancing bird diversity? Insights from community-driven greening projects in Boston. Landscape \& Urban Planning, 114, 69-79.

Sullivan, J. J., \&Molles, L. E. (2016). Biodiversity monitoring by community-based restoration groups in New Zealand. Ecological Management \& Restoration, 17(3), 210-217.

Sullivan, J. J., Meurk, C., Whaley, K. J., \& Simcock, R. (2009). Restoring native ecosystems in urban Auckland: Urban soils, isolation, and weeds as impediments to forest establishment. New Zealand Journal of Ecology, 33(1), 60-71.

Sullivan, J. J., Williams, P. A., \& Timmins, S. M. (2007). Secondary forest succession differs through naturalised gorse and native kānuka near Wellington and Nelson. New Zealand Journal of Ecology, 31(1), 22-38.

Suri, J., Anderson, P. M., Charles-Dominique, T., Hellard, E., \& Cumming, G. S. (2017). More than just a corridor: A suburban river catchment enhances bird functional diversity. Landscape \& Urban Planning, 157, 331-342.

Svoray, T., Bar, P., \& Bannet, T. (2005). Urban land-use allocation in a Mediterranean ecotone: Habitat Heterogeneity Model incorporated in a GIS using a multi-criteria mechanism. Landscape \& Urban Planning, 72(4), 337-351.

Swaffield, S. (2003). Shaping an urban landscape strategy to promote biodiversity. In: Dawson, M., (Ed.), Greening the city: bringing biodiversity into the urban environment, (pp. 246-260), Christchurch, Royal New Zealand Institute of Horticulture.

Swaffield, S. R. (2005). Shaping an urban landscape strategy to promote biodiversity. In Dawson, M. I., (Ed.), Greening the city: Bringing biodiversity back into the urban environment. Proceedings of a Conference held by the Royal New Zealand Institute of Horticulture in Christchurch, 21-24 October 2003, (pp. 248-260), Christchurch: Royal New Zealand Institute of Horticulture.

Syrett, P., Fowler, S. V., Coombs, E. M., Hosking, J. R., Markin, G. P., Paynter, Q. E., \& Sheppard, A. W. (1999). The potential for biological control of Scotch broom (Cytisus scoparius)(Fabaceae) and related weedy species. Biocontrol News \& Information, 20(1), 17-34.

Szlavecz K., Warren, P., \& Pickett, S. (2011). Biodiversity on the urban landscape. In: Concotta R. P., Gorenflo L., J., (Eds.), Human populations: its influences on biological diversity, (pp. 75-101), Berlin: Springer.

Tan, P. Y., \& Jim, C., Y. (2017). Greening Cities: Forms and Functions. Singapore: Springer.

Tan, Z., Lau, K. K. L., \& Ng, E. (2016). Urban tree design approaches for mitigating daytime urban heat island effects in a high-density urban environment. Energy \& Buildings, 114, 265-274.

Taylor S., Castro, I., \& Griffiths, R. (2005). Hihi/stitchbird (Notiomystiscincta) recovery plan 2004-09. Threatened Species Recovery Plan 54. Wellington: Department of Conservation.

Taylor, S., \& Kumar, L. (2016). Global climate change impacts on pacific islands terrestrial biodiversity: a review. Tropical Conservation Science, 9(1), 203-223.

Temple, S.A. (1986). Predicting impacts of habitat fragmentation on Forest birds: a comparison of two models. In: Verbe, J., Morrison, M. L., Ralph, C. J., (Eds.), Modelling Habitat Relationships of Terrestrial Vertebrates, (pp. 301-304), Madison: University of Wisconsin Press.

Tennyson, A. J. D., \& Martinson, P. (2006). Extinct birds of New Zealand. Wellington: Te Papa Press.

Thomas, C. D., Cameron, A., Green, R. E., Bakkenes, M., Beaumont, L. J., Collingham, Y. C., ... \& Hughes, L. (2004). Extinction risk from climate change. Nature, 427(6970), 145-148.

Thorsen, M. I. K. E., Innes, J. O. H. N., Nugent, G. R. A. H. A. M., \& Prime, K. E. V. I. N. (2004). Parental care and growth rates of New Zealand pigeon (Hemiphaga novaeseelandiae) nestlings. Notornis, 51(3), 136-140. 
Threlfall, C. G., Mata, L., Mackie, J. A., Hahs, A. K., Stork, N. E., Williams, N. S., \& Livesley, S. J. (2017). Increasing biodiversity in urban green spaces through simple vegetation interventions. Journal of Applied Ecology. 54(6), 18741883.

Threlfall, C. G., Williams, N. S., Hahs, A. K., \& Livesley, S. J. (2016). Approaches to urban vegetation management and the impacts on urban bird and bat assemblages. Landscape \& Urban Planning, 153, $28-39$.

Tilman, D., Isbell, F., \& Cowles, J. M. (2014). Biodiversity and ecosystem functioning. Annual Review of Ecology, Evolution, \& Systematics, 45(1), 471.

Towns, D. R., \& Daugherty, C. H. (1994). Patterns of range contractions and extinctions in the New Zealand herpetofauna following human colonisation. New Zealand Journal of Zoology, 21(4), 325-339.

Towns, D. R., \& Williams, M. (1993). Single species conservation in New Zealand: towards a redefined conceptual approach. Journal of the Royal Society of New Zealand, 23(2), 61-78.

Turner, M. G. (1989). Landscape ecology: the effect of pattern on process. Annual Review of Ecology \& Systematics, 20(1), 171-197.

Turner, M. G., Gardner, R. H., \& O'neill, R. V. (2001). Landscape ecology in theory and practice (Vol. 401). New York: Springer.

Tzoulas, K., Korpela, K., Venn, S., Yli-Pelkonen, V., Kaźmierczak, A., Niemela, J., \& James, P. (2007). Promoting ecosystem and human health in urban areas using Green Infrastructure: A literature review. Landscape \& Urban Planning, 81(3), 167-178.

UNFCCC (1992). United Nations Framework Convention on Climate Change. Geneva: UNEP/IUC.

United Nations - Department of Economic and Social Affairs, Population Division (2014). World urbanization prospects: the 2014 revision, Highlights (ST/ESA/SER.A/352).

United Nations (2015). World Urbanization Prospects: The 2014 Revision, Department of Economic and Social Affairs, Population Division (ST/ESA/SER.A/366).

United Nations_-Department of Economic and Social Affairs, Population Division. (2008). World urbanization prospects: the 2007 revision. CD-ROM Edition, data in digital form (POP/DB/WUP/Rev.2007).

Urban, M. C. (2015). Accelerating extinction risk from climate change. Science, 348(6234), 571-573.

Uuemaa, E., Mander, Ü.,\&Marja, R. (2013). Trends in the use of landscape spatial metrics as landscape indicators: a review. Ecological Indicators, 28, 100-106.

Vallejo, B. M., Aloy, A. B., \& Ong, P. S. (2009). The distribution, abundance and diversity of birds in Manila's last greenspaces. Landscape \& Urban Planning, 89(3), 75-85.

van den Brink, A., \& Bruns, D. (2014). Strategies for enhancing landscape architecture research. Landscape Research, 39(1), $7-20$.

van den Brink, A., Bruns, D., Tobi, H. \& Bell, S. (2017). Research in Landscape Architecture: Methods and Methodology. London: Routledge.

van Heezik, Y., \& Seddon, P. J. (2017). Counting birds in urban areas: a review of methods for the estimation of abundance. In: Murgui, E., \& Hedblom, M., (Eds.), Ecology and Conservation of Birds in Urban Environments, (pp. 185-207), Springer: Heidelberg.

van Heezik, Y., Freeman, C., Porter, S., \& Dickinson, K. J. (2013). Garden size, householder knowledge, and socioeconomic status influence plant and bird diversity at the scale of individual gardens. Ecosystems, 16(8), 1442-1454. 
van Heezik, Y., Ludwig, K., Whitwell, S., \& McLean, I. G. (2008 b). Nest survival of birds in an urban environment in New Zealand. New Zealand Journal of Ecology, 32(2), 155-165.

van Heezik, Y., Smyth, A., \& Mathieu, R. (2008 a). Diversity of native and exotic birds across an urban gradient in a New Zealand city. Landscape \& Urban Planning, 87(3), 223-232.

van Heezik, Y., Smyth, A., Adams, A., \& Gordon, J. (2010). Do domestic cats impose an unsustainable harvest on urban bird populations?. Biological Conservation, 143(1), 121-130.

van Hove, L. W. A., Jacobs, C. M. J., Heusinkveld, B. G., Elbers, J. A., Van Driel, B. L., \& Holtslag, A. A. M. (2015). Temporal and spatial variability of urban heat island and thermal comfort within the Rotterdam agglomeration. Building \& Environment, 83, 91-103.

van Wilgen, B. W., Esler, K. J., Gaertner, M., Midgley, G. F., Richardson, D. M., van Wilgen, N. J., ... \& Palmer, G. (2016). Ecological research and conservation management in the Cape Floristic Region between 1945 and 2015: History, current understanding and future challenges. Transactions of the Royal Society of South Africa, 71(3), 207-303.

Veblen, T. T., \& Ashton, D. H. (1982). The regeneration status of Fitzroyacupressoides in the Cordillera Pelada, Chile. Biological Conservation, 23(2), 141-161.

Veijalainen, N., Lotsari, E., Alho, P., Vehviläinen, B., \& Käyhkö, J. (2010). National scale assessment of climate change impacts on flooding in Finland. Journal of Hydrology, 391(3), 333-350.

Villavicencio, N. A., Lindsey, E. L., Martin, F. M., Borrero, L. A., Moreno, P. I., Marshall, C. R., \& Barnosky, A. D. (2016). Combination of humans, climate, and vegetation change triggered Late Quaternary megafauna extinction in the Última Esperanza region, southern Patagonia, Chile. Ecography, 39(2), 125-140.

Villegas, M., \& Garitano-Zavala, Á. (2010). Bird community responses to different urban conditions in La Paz, Bolivia. Urban Ecosystems, 13(3), 375-391.

Wadt, L. H. O., Kainer, K. A., Staudhammer, C. L., \& Serrano, R. O. P. (2008). Sustainable forest use in Brazilian extractive reserves: natural regeneration of Brazil nut in exploited populations. Biological Conservation, 141(1), 332-346.

Walker, L. R., Walker, J., \& Hobbs, R. J. (2007). Linking restoration and ecological succession. Berlin: Springer.

Walker, S., Price, R., \& Rutledge, D. T. (2008). New Zealand's remaining indigenous cover: recent changes and biodiversity protection needs. Wellington: Department of Conservation.

Walker, S., Price, R., Rutledge, D., Stephens, R. T., \& Lee, W. G. (2006). Recent loss of indigenous cover in New Zealand. New Zealand Journal of Ecology, 30, 169-177.

Walther, G. R., Post, E., Convey, P., Menzel, A., Parmesan, C., Beebee, T. J., ... \& Bairlein, F. (2002). Ecological responses to recent climate change. Nature, 416(6879), 389-395.

Walz, U., \& Syrbe, R. U. (2013). Linking landscape structure and biodiversity. Ecological Indicators, 31, 1-5.

Walz, U., Hoechstetter, S., Drăguţ, L., \& Blaschke, T. (2016). Integrating time and the third spatial dimension in landscape structure analysis. Landscape Research, 41(3), 279-293.

Wang, X., \& Hofe, R. (2008). Research methods in urban and regional planning. Tsinghua University Texts. Beijing and Berlin: Tsinghua University Press and Springer-Verlag.

Wang, X., Blanchet, F. G., \& Koper, N. (2014). Measuring habitat fragmentation: an evaluation of landscape pattern metrics. Methods in Ecology \& Evolution, 5(7), 634-646.

Wang, Z. H., Zhao, X., Yang, J., \& Song, J. (2016). Cooling and energy saving potentials of shade trees and urban lawns in a desert city. Applied Energy, 161, 437-444. 
Wardell-Johnson, G., Wardell-Johnson, A., Bradby, K., Robinson, T., Bateman, P. W., Williams, K., ... \& Burbridge, M. (2016). Application of a Gondwanan perspective to restore ecological integrity in the south-western Australian global biodiversity hotspot. Restoration Ecology, 24(6), 805-815.

Watts, C., Thornburrow, D., Cave, V., \& Innes, J. (2014). Beetle community changes following pest mammal control at two biodiversity sanctuaries in Wellington, New Zealand. Journal of the Royal Society of New Zealand, 44(2-3), 61-87.

Webb, C. J., \& Kelly, D. (1993). The reproductive biology of the New Zealand flora. Trends in Ecology \& Evolution, 8(12), 442-447.

Webb, C. J., Sykes, W. R., \& Garnock-Jones, P. J. (1988). Flora of New Zealand, vol. IV. Botany Division, Christchurch: DSIR.

Wellington City Council (2015). Wellington Urban Growth Plan. Wellington: Wellington City Council.

Wellington City Council Open Data. (2015). Wellington City Council Boundary. Accessed at: http://data.wcc.opendata.arcgis.com

Western, D. (1992). The biodiversity crisis: a challenge for biology. Oikos, 63(1), 29-38.

Whitaker, A. H. (1987). The roles of lizards in New Zealand plant reproductive strategies. New Zealand Journal of Botany, 25(2), 315-328.

Wilby, R. L., \& Perry, G. L. (2006). Climate change, biodiversity and the urban environment: a critical review based on London, UK. Progress in Physical Geography, 30(1), 73-98.

Williams, P. A. (2011). Secondary succession through non-native dicotyledonous woody plants in New Zealand. New Zealand Natural Sciences, 36, 73-91.

Williams, P. A., \& Timmins, S. (2002). Economic impacts of weeds in New Zealand (pp. 175-184). Boca Raton: CRC Press.

Williams, P. A., Karl, B. J., Bannister, P., \& Lee, W. G. (2000). Small mammals as potential seed dispersers in New Zealand. Austral Ecology, 25(5), 523-532.

Williams. P. A. (2003). Are possums important dispersers of large-seeded fruit? New Zealand Journal of Ecology, 27: 221223.

Wilson, C. M., \& Given, D. R. (1989). Threatened Plants of New Zealand. Wellington: DSIR Publishing.

Wilson, K. J. (2004). Flight of the Huia: Ecology and conservation of New Zealand's frogs, reptiles, birds and mammals. Christchurch: Canterbury University Press.

Wilson, P. R., Karl, B. J., Toft, R. J., Beggs, J. R., \& Taylor, R. H. (1998). The role of introduced predators and competitors in the decline of kaka (Nestor meridionalis) populations in New Zealand. Biological Conservation, 83(2), 175-185.

Wiser, S. K., Allen, R. B. (2006). What controls invasion of indigenous forests by alien plants? In: Allen R. B. \& Lee W. G. (Eds). Biological invasions in New Zealand, (pp. 195-209), Berlin, Springer-Verlag.

Wodzicki, J. (1950). Introduced mammals of New Zealand. An ecological and economic survey. Wellington: DSIR.

Wotton D. M. (2007). Consequences of dispersal failure: kereru and large seeds in New Zealand. Doctoral Thesis: University of Canterbury, Christchurch, New Zealand.

Wotton, D. M. (2002). Effectiveness of the common gecko (Hoplodactylus maculatus) as a seed disperser on Mana Island, New Zealand. New Zealand Journal of Botany, 40(4), 639-647.

Wotton, D. M., \& Kelly, D. (2012). Do larger frugivores move seeds further? Body size, seed dispersal distance, and a case study of a large, sedentary pigeon. Journal of Biogeography, 39(11), 1973-1983. 
Wotton, D. M., \& McAlpine, K. G. (2013). Predicting native plant succession through woody weeds in New Zealand. Wellington: Department of conservation.

Wotton, D. M., \& McAlpine, K. G. (2015). Seed dispersal of fleshy-fruited environmental weeds in New Zealand. New Zealand Journal of Ecology, 39(2), 155-169.

Wotton, D. M., Drake, D. R., Powlesland, R. G., \& Ladley, J. J. (2016). The role of lizards as seed dispersers in New Zealand. Journal of the Royal Society of New Zealand, 46(1), 40-65.

Wu, J. (2014). Urban ecology and sustainability: The state-of-the-science and future directions. Landscape \& Urban Planning, 125, 209-221.

Wyman, T. E., \& Kelly, D. (2017). Quantifying seed dispersal by birds and possums in a lowland New Zealand forest. New Zealand Journal of Ecology, 41(1), 47-55.

Xiao, J., Shen, Y., Ge, J., Tateishi, R., Tang, C., Liang, Y., \& Huang, Z. (2006). Evaluating urban expansion and land use change in Shijiazhuang, China, by using GIS and remote sensing. Landscape \& Urban Planning, 75(1), 69-80.

Yli-Pelkonen, V., \& Niemelä, J. (2006). Use of ecological information in urban planning: Experiences from the Helsinki metropolitan area, Finland. Urban Ecosystems, 9(3), 211-226.

Young, A., \& Mitchell, N. (1994). Microclimate and vegetation edge effects in a fragmented podocarp-broadleaf forest in New Zealand. Biological Conservation, 67(1), 63-72.

Yuan, B., \& Lu, C. (2016). Effects of urbanization on bird diversity: a case study in Yizhou, Guangxi Province, China. Asia Life Sciences, 25(1), 79-96.

Zheng, B., Myint, S. W., \& Fan, C. (2014). Spatial configuration of anthropogenic land cover impacts on urban warming. Landscape \& Urban Planning, 130, 104-111.

Zhou, D., \& Chu, L. M. (2012). How would size, age, human disturbance, and vegetation structure affect bird communities of urban parks in different seasons?. Journal of Ornithology, 153(4), 1101-1112.

Zhou, W., Huang, G., \& Cadenasso, M. L. (2011). Does spatial configuration matter? Understanding the effects of land cover pattern on land surface temperature in urban landscapes. Landscape \& Urban Planning, 102(1), 54-63.

Ziter, C. (2016). The biodiversity-ecosystem service relationship in urban areas: a quantitative review. Oikos, 125(6), 761768.

Zucca, A., Sharifi, A. M., \& Fabbri, A. G. (2008). Application of spatial multi-criteria analysis to site selection for a local park: A case study in the Bergamo Province, Italy. Journal of Environmental Management, 88(4), 752-769. 


\section{Appendix I The questionnaire used in Study I}

\section{PART A: Introduction}

I am a $\mathrm{PhD}$ candidate in Landscape Architecture from Victoria University of Wellington, New Zealand. My $\mathrm{PhD}$ research is titled "Urban biodiversity in an era of climate change: towards an optimised landscape pattern in support of indigenous wildlife species in urban New Zealand”. To have a better picture of what I will be asking you in this questionnaire, please read PART B in advance of addressing the questions. Answering all questions is optional. While it should take you no more than ten to twenty minutes to answer all questions, your contribution will be extremely helpful to me to successfully conduct this part of my research. Please feel free to get in touch with me or my supervisors if you have any questions regarding this questionnaire.

Kind regards

Amin Rastandeh

PhD candidate in Landscape Architecture

School of Architecture, Victoria University of Wellington, New Zealand

\section{PART B: Background}

In a world where natural resources are under stress from climate change and rapid urbanisation, biodiversity must be taken into particular consideration because biodiversity loss affects ecosystem services and this in turn can have far-reaching consequences for humans in the long run. Research shows that the risk of biodiversity loss from climate change in South America, Australia and New Zealand is higher than for other parts of the world. There is evidence that biodiversity often can be rich in urban areas and also evidence that the change in components of landscape pattern has implications for both urban biodiversity targets and climate change adaptation goals. Similarity in dependency on landscape pattern, however, does not mean that wildlife species and humans always require a similar landscape pattern to be safeguarded against the ill effects of climate change. Any change in compositional or configurational components of landscape pattern may have different implications for urban biodiversity targets and/or climate change adaptation goals. In some cases, what wildlife species require to be safeguarded against the changing climate may differ widely from what humans need in the urban environment in order to tackle the negative effects of climate change.

The main aim of this expert survey is to gather a range of data that is unlikely to be found in the current literature. Then the results will be scrutinized in the second study to corroborate them and confirm their suitability to be applied in a New Zealand context. Combined with other types of data, the final outputs will be used to construct a multi-dimensional dataset in order to undertake the final stage of this research.

\section{PART C: General questions}

Please answer the questions based on your experience in the specific geographic area where you have worked/are working. Please note that answering all questions is optional.

C1: Academic affiliation (e.g. Victoria University of Wellington)

C2: Have you conducted any research on the connection between landscape pattern composition and configuration and urban wildlife species in recent years?

YES

NO (go to PART F)

C3: Please specify the countries in which you have conducted your previous research since 2000. 
C4: Please specify the number of research projects you have undertaken on this topic since 2000.

C5: Please specify the scale of study you have undertaken. You may choose more than one option.

\section{Part D: Components of landscape pattern affecting wildlife species}

You can refer to Part $\mathrm{H}$ to see the definitions of the terms used in this part. Please note that answering all questions is optional.

D1: Please score each of the eight components of landscape pattern listed below, using a score from 1 the least important, to 5 the most important, in terms of its unique influence on urban wildlife species in an era of climate change. For example, if you believe that patch size plays a pivotal role in affecting urban biodiversity in an era of climate change, give it a score of 5 and conversely, if you believe that item has the least important role, give it a score of 1.

\section{Glossary}

Edge contrast: dissimilarity between one patch with another in their borders.

Edge density: the presence and quantity of the patch edge across the landscape

Edge effect: climatic and/or human caused effects penetrating into patch along its edge.

Indigenousness: the state of being indigenous/native in terms of land cover.

Land cover heterogeneity: diversity of different land cover type classes in patch or landscape levels.

Landform diversity: diversity of elevations, slopes, and aspects.

Components of landscape pattern affecting wildlife species in urbanised landscapes

\begin{tabular}{|l|c|c|c|c|c|}
\hline \multirow{2}{*}{ Components } & Least important & Less important & Intermediate & More important & Most important \\
\cline { 2 - 6 } & $\mathbf{1}$ & $\mathbf{2}$ & $\mathbf{3}$ & $\mathbf{4}$ & $\mathbf{5}$ \\
\hline Indigenousness & & & & & \\
\hline Land cover heterogeneity & & & & & \\
\hline Land surface perviousness & & & & & \\
\hline Patch size & & & & & \\
\hline Connectivity and proximity & & & & & \\
\hline Edge density and contrast & & & & & \\
\hline Landform diversity & & & & & \\
\hline Shape complexity & & & & & \\
\hline
\end{tabular}

D2: Do you want to add another item to the list of components of landscape pattern presented in D1?

YES (go to D3).

NO (go to PART E).

D3: Please specify additional component(s) of landscape pattern affecting wildlife species and then give reason(s) to justify why you think that the introduced component(s) is/are important to be addressed.

D4: Dedicate an appropriate score to what you have listed in D3, from 1 the least important, to 5 the most important, in terms of its unique influence on wildlife species in an era of climate change.

\section{PART E: Areas of conflict}

Please answer the following questions based on your experience and observations made in the course of your previous research. Please take climate change into particular consideration when addressing the following four questions. There is no word limit in this part. Please note that answering all questions is optional.

E1: Indigenous or exotic?

While indigenous plant species are regarded as an essential for ecological integrity over time, some exotic plants are commonplace species for carbon sequestration and storage, and are often economically and socially acceptable. According to what you have found in the course of research and practice, is it possible to find a middle ground to alleviate the current conflict between indigenous and exotic species?

E2: Isolation or connection? 
While isolated patches of vegetation may be suggested to reduce the chance of pest and weed dispersal, connected patches of vegetation may facilitate the movement of species from one patch to another for foraging, breeding, roosting, etc.

In an era of climate change, connected patches of vegetation can reduce the urban heat island effect through increasing the cooling effects of urban green spaces. Conversely, isolated patches of vegetation may be regarded as safe havens for wildlife species to be safeguarded against the spread of extreme weather events such as flood, heat waves, etc. According to what you have found in the course of research and practice, is it possible to find a middle ground to alleviate the current conflict between spatial isolation and connection?

E3: High and low edge density and contrast

While low edge density can be regarded as a spatial strategy for reducing climate-related edge effects in support of wildlife species in the face of extreme weather events, high edge density can reduce the urban heat island effect through increasing the cooling effects of urban green spaces. At the same time, while high edge contrast may be a physical barrier to climate change-induced impacts such as disease spread, pest and weed dispersal, wildfire, etc., it may provide restrictions for wildlife species to move from one patch to another for foraging, breeding, roosting, etc. According to what you have found in the course of research and practice, is it possible to find a middle ground to alleviate the current conflict between high and low edge density and contrast?

\section{E4: Spatially compacted or dispersed}

While compact patches of vegetation may be considered as a means for reducing negative impacts on wildlife species through providing a larger core area protected from climatic edge effects, research shows that irregular extensive patches of vegetation can be beneficial for reducing the impacts of urban heat island in urban environments. According to what you have found in the course of research and practice, is it possible to find a middle ground to alleviate the current conflict between spatially compacted and dispersed configurations?

\section{PART F: Snowball approach}

Please forward the link of this questionnaire to scientists and/or researchers who may be able to contribute to this research.

\section{Part G: Acknowledgement}

Thank you very much for devoting your valuable time to contribute to my research. I would be extremely grateful to you for any further feedback regarding the topic under investigation. Please feel free to get in touch with me or my supervisors, if you have any questions and/or suggestions. You may leave a comment below, if you wish. 


\section{Appendix III Questions addressed in Study II}

\section{General information}

The main aim of this interview is to gather a range of data that is unlikely to be found in the current literature. The questions have been designed based on the available data published in the current literature and the results of an international survey conducted from 25 October 2016 to 24 January 2017 using an online survey tool. The outputs will be used in the process of spatial analysis of landscape pattern composition and configuration in Wellington, New Zealand.

\subsection{Climate change and pattern process relationship model}

In a world where natural resources are under stress from climate change and rapid urbanisation, biodiversity must be taken into particular consideration because biodiversity loss affects ecosystem services, and this in turn can have far-reaching consequences for humans in the long run. Research shows that the risk of biodiversity loss from climate change in South America, Australia and New Zealand is higher than other parts of the world. Despite this, as biodiversity can be often rich in urban areas some believe that such a confluence can simultaneously open up new opportunities to safeguard wildlife species against the ill effects of climate change. There is evidence that the change in components of landscape pattern has implications for urban biodiversity targets in urban environments. Spatial patterning of landscapes has a widespread influence on urban wildlife species richness and abundance. Recent evidence demonstrates that survival of urban wildlife species depends, to a large extent, on composition and configuration of land cover type classes cumulatively shaping landscape pattern.

\subsection{Local impacts of climate change}

According to the Ministry for the Environment (2014), projected impacts of climate change on Wellington are very likely to be:

(1) A $0.9^{\circ} \mathrm{C}$ to $2.1^{\circ} \mathrm{C}$ increase in annual average temperature by 2040 and 2090, respectively compared to 1990 ;

(2) A 2\% to $5 \%$ increase in the frequency of extreme winds in winter and a decrease by a similar amount during summer;

(3) An increase in the frequency of heavy rainfall events during southerly storms;

(4) Increase and decrease in the number of storms crossing the Tasman Sea during summer and winter, respectively;

(5) A mean sea-level rise of $80 \mathrm{~cm}$ by the end of the century.

\subsection{Keystone species in the study area}

Since landscape ecology research is extremely site and species-specific (Turner, 1989; Ahern, 1999; Botequilha Leitao and Ahern, 2002; Botequilha Leitao et al, 2006), it is difficult, in practice, to define a framework to encompass all species when studying urban biodiversity. Nevertheless, it is reasonable to address a smaller range of keystone species whose roles in urban ecosystems support the survival of other species that remain outside the study. By definition, keystone species are "species whose activities are critical to the structure of the community in which they live" (Collier et al, 1997, 18). Research shows that disappearance of keystone species can affect the entire ecosystem over time (q.v. Mills et al, 1993). Garibaldi and Turner (2004) argued that culturally-important species can also be considered as keystone species due to their iconic significance. Payton et al (2002) identified four types of keystone species relevant to New Zealand, namely organisms controlling potential dominants, resource providers, mutualists, and ecosystem engineers. They argue that there is a considerable number of keystone species in New Zealand ecosystems (q.v. Payton et al, 2002). In this research ecological and cultural criteria are considered to define indigenous urban keystone species. While a declining number of keystone species contributes to widespread urban biodiversity loss, recognising suitable habitats for keystone species may help to 
ensure urban biodiversity in the face of climate change and rapid urbanisation not only for them, but also for a wide range of other species. Based upon the current literature (e.g. Lloyd, 1985; Whitaker, 1987; Clout and Hay 1989; Towns and Williams, 1993; Webb and Kelly, 1993; Bell, 1996; Payton, 2002; Wotton, 2002; Chambers, 2004; Dymond and Shepherd, 2004; Anderson et al., 2006; Boersma, 2008; Foster, 2008; Anderson et al, 2011; Kunz et al, 2011; Grayson et al, 2014), the research has provided a list of keystone species present and/or having the potential to be present in the study area (Table 1).

\subsection{Glossary}

Edge contrast: dissimilarity between one patch with another in their borders.

Edge density: the presence and quantity of the patch edge across the landscape.

Edge effect: climatic and/or human caused effects penetrating into patch along its edge.

Indigenousness: the state of being indigenous/native in terms of land cover.

Land cover heterogeneity: diversity of different land cover type classes in patch or landscape levels.

Landform diversity: diversity of elevations, slopes, and aspects.

\section{Interview questions}

\subsection{QUESTION 1}

In my earlier research, the most important components of landscape pattern affecting wildlife species in urban environments have been listed below. They have been ranked by international scientists and researchers in terms of importance to wildlife species through a global questionnaire-centred expert survey.

Components of landscape pattern (Rankings will be provided in the interview session).

- Indigenousness

- Land cover heterogeneity

- $\quad$ Land surface perviousness

- Patch size

- Connectivity and proximity

- $\quad$ Edge density and contrast

- Landform diversity

- $\quad$ Shape complexity

With a particular emphasis on indigenous keystone species currently present in the study area and the estimated impacts on the selected wildlife species attributed to climate change in the current literature (Table 1), do you think that the results derived from the international survey are relevant to a New Zealand context and can be applied in urban New Zealand? If so, in what way?

\subsection{QUESTION 2}

Do you consider that any other keystone species not listed in Table 1 deserve to be included? If so which ones and why?

\subsection{QUESTION 3}

Of 33 land cover types (LCTs) in the NZ environment, 16 LCTs are now present in the study area. On a landscape scale, do you think that the study area is capable of a greater LCT diversity? If yes, please explain how LCT diversity can be increased and what types of land cover can be restored or regenerated in the study area. A list of New Zealand LCT classes will be provided in the interview session. Alternatively, you can see this list at: https://ris.scinfo.org.nz/layer/423-lcdb-v41-landcover-database-version-41-mainland-new-zealand/ 
Table 1

Documented impacts of climate change on the selected collection of indigenous wildlife/keystone species in urban New Zealand $^{62}$

Keystone species present in the study area

Long-tailed bat

Indigenous birds

e.g. Tūī, Bellbird, Hihi, Kererū, Little-spotted kiwi

Tuatara

Lizards and skinks

e.g. Forest gecko, Green gecko
Impacts attributed to climate change in the scientific literature

- $\quad$ Foraging source shortage in warmer winters when the species are likely to be more active than in climatically ordinary winters (Pryde et al., 2005).

- $\quad$ Predator pressure due to mast seeding phenomenon caused by rising temperature (McGlone and Walker, 2011; Christie, 2014).

- $\quad$ Increased edge effects due to wind penetration into habitat core area (Yang and Mitchell, 1984; Davies-Colley et al., 2000).

- $\quad$ Predator pressure due to mast seeding phenomenon caused by rising temperature (McGlone and Walker, 2011; Christie, 2014).

- $\quad$ An increase in temperate is likely to adversely affect hihi population dynamics (Chauvenet et al. 2013).

- Misbalance between male and female populations due to warmer than average temperatures (Grayson et al., 2014).

- $\quad$ Habitat destruction due to intensified winds and heavy rainfall (Romijn et al., 2012).

- $\quad$ Predator pressure due to mast seeding phenomenon caused by rising temperature (McGlone and Walker, 2011; Romijn et al., 2012; Christie, 2014).

- $\quad$ Habitat destruction due to sea level rise, more intensified winds, heavy rainfall, and coastal erosion (Davis, 1994; Melville and Battley, 2006; Ray, 2013).

- $\quad$ Predator pressure due to mast seeding phenomenon caused by rising temperature (Wilson et al., 1998; Pierce et al., 2006).

- Warmer than average temperatures leads to delayed breeding in some seabirds (Chambers, 2004).

62. This Table was revised and developed in the course of the research (cf. Table 1.5). 
Table 2

Examples of probable consequences of local impacts of climate change on wildlife habitats in the Wellington urban landscape.

Climate change impacts

Direct impacts

- Wildlife habitats specifically along edges are subject to extreme weather events such as intensified windstorms;

- Some urban wildlife habitats are susceptible to extreme weather events triggered by rising temperatures and intensified windstorms, specifically along edges;

- $\quad$ Low-land areas are susceptible to flooding and inundation affecting remaining habitats within the city;

- Wildlife habitats may get warmer, specifically in north-facing slopes providing unsuitable conditions for indigenous fauna historically adapted to normal temperatures.

- $\quad$ Large areas of urban costal habitats are subject to sea level rise affecting seabirds;

Indirect impacts

- $\quad$ Extreme weather events such as intensified windstorms and flooding events may facilitate the increased dispersal of weeds and pests affecting indigenous flora and consequently fauna, specifically along edges;

- $\quad$ Rising temperatures may benefit exotic fauna, particularly predators of indigenous fauna. This may increase the rate of predation upon New Zealand avifauna, and reptiles;

- More frequent heavy rainfalls can give rise to more landslides which will destroy or damage wildlife habitats specifically in areas where landform diversity can harbour indigenous fauna;

- The outbreak and spread of diseases in avifauna is likely to be higher in warmer than average temperatures.

- Sea level rise may reinforce the need to relocate different types of urban infrastructure (e.g. industrial, commercial, recreational, etc.) to new open spaces along the water bodies and this could negatively affect indigenous species that depend on coastal habitats;

\subsection{QUESTION 4}

Please complete the dissimilarity matrix based on your empirical experience of different land cover types (see below). Please indicate a dissimilarity (edge contrast) weight, using a score from 1.0, the highest edge contrast, to 0.0 , the lowest edge contrast, in terms of its unique influence on the selected keystone species with a particular attention to the local impacts of climate change (as listed in Table 1). For example, based on your empirical experience, if you believe that the dissimilarity between Type A and Type $\mathrm{C}$ is high, give a score of 1.0 and conversely, if you believe that the dissimilarity has minimal or no negative effect on keystone species, give a score of 0.0. Please use the spectrum below to give dissimilarity (edge contrast) weights:

\section{Scoring scale}

\begin{tabular}{|l|l|l|l|l|l|l|l|l|l|l|}
\hline 0.0 & 0.1 & 0.2 & 0.3 & 0.4 & 0.5 & 0.6 & 0.7 & 0.8 & 0.9 & 1.0 \\
\hline
\end{tabular}

\section{Type A: Indigenous}

Broadleaved Indigenous Hardwoods

Indigenous Forest

Matagouri or Grey Scrub

Herbaceous Freshwater Vegetation

\section{Type B: Non-indigenous}

Gorse and/or Broom

High Producing Exotic Grassland

Exotic Forest

Urban Parkland/Open Space

Low Producing Grassland

Forest Harvested

\section{Type C: Extremely human-modified}

Built-Up Area

Transport Infrastructure

Surface Mine or Dump 


\section{Type D: Other}

Sand or Gravel

Lake or Pond

Gravel or Rock

\section{Dissimilarity Matrix}

\begin{tabular}{|l|l|l|l|l|}
\hline Type A & 0 & 0 & 0 & 0 \\
\hline Type B & & 0 & 0 & 0 \\
\hline Type C & & & 0 & 0 \\
\hline Type D & & & & 0 \\
\hline & Type A & Type B & Type C & Type D \\
\hline
\end{tabular}

Note: If you believe that there are dissimilarities within each group, please specify and then give an appropriate score.

\subsection{QUESTION 5}

As mentioned above, projected impacts of climate change on Wellington are very likely to be:

(1) A $0.9^{\circ} \mathrm{C}$ to $2.1^{\circ} \mathrm{C}$ increase in annual average temperature by 2040 and 2090, respectively compared to 1990 ;

(2) A $2 \%$ to $5 \%$ increase in the frequency of extreme winds in winter and a decrease by a similar amount during summer;

(3) An increase in the frequency of heavy rainfall events during southerly storms;

(4) Increase and decrease in the number of storms crossing the Tasman Sea during summer and winter, respectively;

(5) A mean sea-level rise of $80 \mathrm{~cm}$ by the end of the century (q.v. Ministry for the Environment, 2014).

Based upon the information summarised in Table 1 (see Question 1) and Table 2 (see Question 3), please rank the estimated impacts of climate change based on the depth and extensiveness of the impacts on the selected keystone species mentioned above.

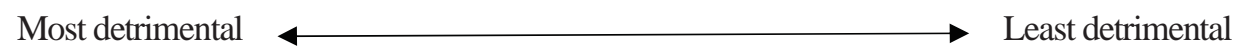

\subsection{QUESTIONS 6}

With particular attention to the selected keystone species' ecological requirements as well as the estimated impacts of climate change on them, do you agree with these spatial thresholds (see Table 3) as a basis for judgement about the capability of the current landscape pattern composition and configuration in safeguarding indigenous biodiversity in the study area?

\section{Table 3}

Spatial thresholds proposed for supporting indigenous biodiversity in urban New Zealand (Meurk and Hall, 2006) linked to components of landscape pattern defined in the current research.

\begin{tabular}{l} 
Components of landscape pattern \\
Indigenousness \\
Land cover heterogeneity \\
Land surface perviousness \\
Patch size \\
Connectivity and proximity \\
Edge density and contrast \\
Landform diversity \\
Shape complexity \\
\hline
\end{tabular}

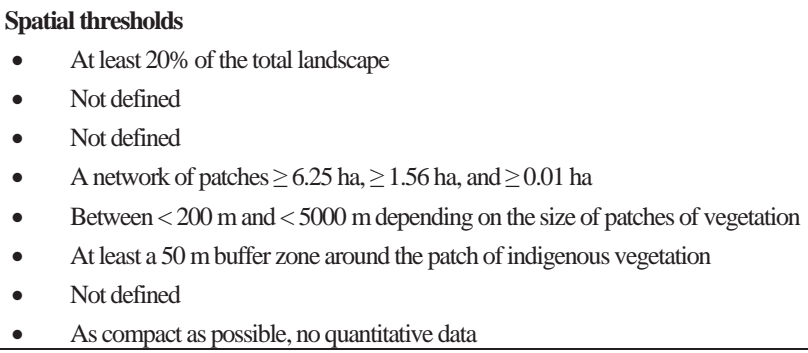

More information regarding Table 3 will be provided in the interview session. Please fill in Table 4 compared to Table 3. 
Table 4

\begin{tabular}{|c|c|c|}
\hline Components of landscape pattern & Acceptable Yes/No & Proposed spatial thresholds \\
\hline \multicolumn{3}{|l|}{ Indigenousness } \\
\hline \multicolumn{3}{|l|}{ Land cover heterogeneity } \\
\hline \multicolumn{3}{|l|}{ Land surface perviousness } \\
\hline \multicolumn{3}{|l|}{ Patch size } \\
\hline \multicolumn{3}{|l|}{ Connectivity and proximity } \\
\hline \multicolumn{3}{|l|}{ Edge density and contrast } \\
\hline \multicolumn{3}{|l|}{ Landform diversity } \\
\hline Shape complexity & & \\
\hline
\end{tabular}

\section{Opportunities for revision}

\subsection{Note 1:}

After converting each interview into text, a summarised written version of it will be returned to you for revision. You will have the opportunity to revise your statements within 15 days. Would you like to receive a summarised written version of your interview for revision?

YES (go to Section 3.2)

$\mathrm{NO}$ (go to Section 4)

\subsection{Note 2:}

The final results stemming from this study will be sent to all interviewees for final revision. You will have an opportunity to compare your responses to those of others, anonymously. Would you like to receive the final results anonymously including your own responses?

YES

NO

\section{Acknowledgement}

Thank you very much for devoting your valuable time to contribute to my research. I would be extremely grateful to you if you give me further feedback regarding the topic under investigation. Please feel free to get in touch with me or my supervisor, Dr Maibritt Pedersen Zari, if you have any questions and/or suggestions.

\section{References}

Literature cited in Appendix III, has been listed in the list of references. 


\section{Appendix IV \\ Ethics approval for Study II}

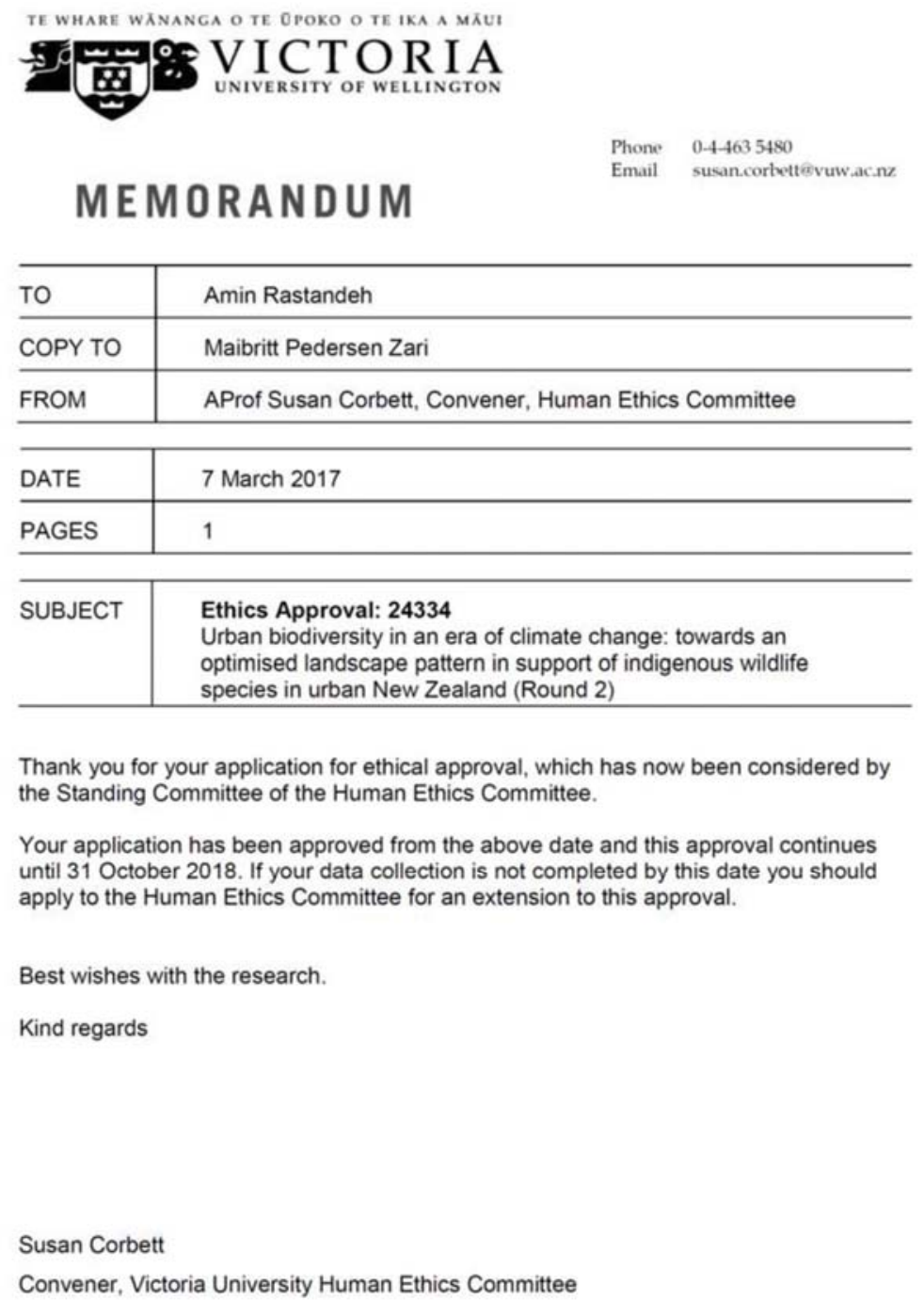

\begin{tabular}{l|l}
\hline TO & Amin Rastandeh \\
\hline COPY TO & Maibritt Pedersen Zari \\
\hline FROM & AProf Susan Corbett, Convener, Human Ethics Committee \\
\hline \multicolumn{2}{|l}{} \\
\hline DATE & 7 March 2017 \\
\hline PAGES & 1 \\
\hline
\end{tabular}

SUBJECT Ethics Approval: 24334

Urban biodiversity in an era of climate change: towards an optimised landscape pattern in support of indigenous wildlife species in urban New Zealand (Round 2)

Thank you for your application for ethical approval, which has now been considered by the Standing Committee of the Human Ethics Committee.

Your application has been approved from the above date and this approval continues until 31 October 2018. If your data collection is not completed by this date you should apply to the Human Ethics Committee for an extension to this approval.

Best wishes with the research.

Kind regards 


\section{Appendix V \\ Land cover classes in the Wellington urban landscape}

\begin{tabular}{|c|c|}
\hline Land cover class & Definition (Landcare Research, 2015) \\
\hline Built-up Area (settlement) & $\begin{array}{l}\text { Commercial, industrial or residential buildings, including associated infrastructure and amenities, not } \\
\text { resolvable as other classes. Low density 'lifestyle’ residential areas are included where hard surfaces, } \\
\text { landscaping and gardens dominate other land covers. }\end{array}$ \\
\hline Urban Parkland/Open Space & $\begin{array}{l}\text { Open, mainly grassed or sparsely-treed, amenity, utility and recreation areas. The class includes parks } \\
\text { and playing fields, public gardens, cemeteries, golf courses, berms and other vegetated areas usually } \\
\text { within or associated with built-up areas. }\end{array}$ \\
\hline Transport Infrastructure & $\begin{array}{l}\text { Artificial surfaces associated with transport such as arterial roads, rail-yards and airport runways. Skid } \\
\text { sites and landings associated with forest logging are sometimes also included. }\end{array}$ \\
\hline Surface Mine or Dump & $\begin{array}{l}\text { Bare surfaces arising from open-cast and other surface mining activities, quarries, gravel-pits and areas } \\
\text { of solid waste disposal such as refuse dumps, clean-fill dumps and active reclamation sites. }\end{array}$ \\
\hline Sand or Gravel & $\begin{array}{l}\text { Bare surfaces dominated by unconsolidated materials generally finer than coarse gravel }(60 \mathrm{~mm}) \text {. } \\
\text { Typically mapped along sandy seashores and the margins of lagoons and estuaries, lakes and rivers and } \\
\text { some areas subject to surficial erosion, soil toxicity and extreme exposure. }\end{array}$ \\
\hline Gravel or Rock & $\begin{array}{l}\text { Bare surfaces dominated by unconsolidated or consolidated materials generally coarser than coarse } \\
\text { gravel (60mm). Typically mapped along rocky seashores and rivers, sub-alpine and alpine areas, scree } \\
\text { slopes and erosion pavements. }\end{array}$ \\
\hline Lake or Pond & $\begin{array}{l}\text { Essentially-permanent, open, fresh-water without emerging vegetation including artificial features such } \\
\text { as oxidation ponds, amenity, farm and fire ponds and reservoirs as well as natural lakes, ponds and } \\
\text { tarns. }\end{array}$ \\
\hline High Producing Exotic Grassland & $\begin{array}{l}\text { Exotic sward grassland of good pastoral quality and vigour reflecting relatively high soil fertility and } \\
\text { intensive grazing management. Clover species, ryegrass and cocksfoot dominate with lucerne and } \\
\text { plantain locally important, but also including lower-producing grasses exhibiting vigour in areas of } \\
\text { good soil moisture and fertility. }\end{array}$ \\
\hline Low Producing Grassland & $\begin{array}{l}\text { Exotic sward grassland and indigenous short tussock grassland of poor pastoral quality reflecting lower } \\
\text { soil fertility and extensive grazing management or non-agricultural use. Browntop, sweet vernal, } \\
\text { danthonia, fescue and Yorkshire fog dominate, with indigenous short tussocks (hard tussock, blue } \\
\text { tussock and silver tussock) common in the eastern South Island and locally elsewhere. }\end{array}$ \\
\hline Herbaceous Freshwater Vegetation & $\begin{array}{l}\text { Herbaceous wetland communities occurring in freshwater habitats where the water table is above or } \\
\text { just below the substrate surface for most of the year. The class includes rush, sedge, restiad, and } \\
\text { sphagnum communities and other wetland species, but not flax nor willows which are mapped as } \\
\text { Flaxland and Deciduous Hardwoods respectively. }\end{array}$ \\
\hline Gorse and/or Broom & $\begin{array}{l}\text { Scrub communities dominated by gorse or Scotch broom generally occurring on sites of low fertility, } \\
\text { often with a history of fire, and insufficient grazing pressure to control spread. Left undisturbed, this } \\
\text { class can be transitional to Broadleaved Indigenous Hardwoods. }\end{array}$ \\
\hline Broadleaved Indigenous Hardwoods & $\begin{array}{l}\text { Lowland scrub communities dominated by indigenous mixed broadleaved shrubs such as wineberry, } \\
\text { mahoe, five-finger, Pittosporum spp, fuchsia, tutu, titoki and tree ferns. This class is usually indicative } \\
\text { of advanced succession toward indigenous forest. }\end{array}$ \\
\hline Matagouri or Grey Scrub & $\begin{array}{l}\text { Scrub and shrubland comprising small-leaved, often divaricating shrubs such as matagouri, Coprosma } \\
\text { spp, Muehlenbeckia spp., Casinnia spp., and Parsonsia spp. These, from a distance, often have a grey } \\
\text { appearance. }\end{array}$ \\
\hline Forest - Harvested & $\begin{array}{l}\text { Predominantly bare ground arising from the harvesting of exotic forest or, less commonly, the clearing } \\
\text { of indigenous forest. Replanting of exotic forest (or conversion to a new land use) is not evident and nor } \\
\text { is the future use of land cleared of indigenous forest. }\end{array}$ \\
\hline Indigenous Forest & Tall forest dominated by indigenous conifer, broadleaved or beech species. \\
\hline Exotic Forest & $\begin{array}{l}\text { Planted or naturalised forest predominantly of radiata pine but including other pine species, Douglas fir, } \\
\text { cypress, larch, acacia and eucalypts. Production forestry is the main land use in this class with minor } \\
\text { areas devoted to mass-movement erosion-control and other areas of naturalised (wildling) } \\
\text { establishment. }\end{array}$ \\
\hline
\end{tabular}




\section{Appendix VI \\ Pre-human forest types in the Wellington urban landscape}

\begin{tabular}{ll}
\hline Land cover class & The spatial extent and definition (Leathwick et al, 2004) \\
\hline Rimu/tawa-kamahi Forest & Central and southern North Island - rimu, miro and totara with less frequent \\
& kahikatea and matai, emergent over a canopy dominated by tawa and kamahi \\
with widespread hinau, rewarewa and mahoe. Pukatea is common, particularly & in valley bottoms, and kohekohe is often abundant at lower elevations except in \\
& drier climates. Black beech is locally common, generally forming discrete \\
enclaves on dry ridges as in hill-country north of Gisborne and inland from & Wanganui.
\end{tabular}

Kahikatea-matai/tawa-mahoe Forest

Dry dune land and low hill country in Hawkes Bay, Manawatu, Wairarapa and coastal Marlborough and Canterbury, including Banks Peninsula - scattered kahikatea and matai with occasional rimu and totara, emergent over a variable canopy of tawa, mahoe, titoki, hinau, maires, pukatea, and puriri. Tarata is locally abundant on Banks Peninsula, but titoki is rare and tawa, maires and puriri are absent.

Lowland forests of wet, cool climates from the Grey Valley north to the Tararua and Aorangi Ranges, with local occurrences in north Taranaki. Scattered rimu, miro and Hall's totara are emergent over a canopy dominated by kamahi, red beech, hard beech and/or silver beech, the latter particularly at higher elevation. Silver beech is of very restricted distribution in north Taranaki. Quintinia and southern rata are much less abundant than where beeches are absent, and mountain beech occurs locally, mostly on sites with skeletal, infertile, or poorly drained soils.

Lowland alluvium and floodplains of North Island and northem and eastern South Island - generally consisting of abundant kahikatea with varying mixtures of other species. The most widespread of the latter include tawa and pukatea, while matai, rimu and totara are generally restricted to better-drained soils. Titoki and puriri are locally abundant, occurring in greatest numbers on poorly drained soils in the drier lowlands of the North Island. The abundance of all of these latter species may have been higher in pre-human forests that predicted here, given the greater ability of kahikatea to regenerate in disturbed or secondary forests, with the latter potentially over-represented in the compositional data from some highly modified landscapes. Kauri probably also once occurred in these forests in the north as far south as the northern Waikato and coastal Bay of Plenty.

Rimu-matai-miro-totara/kamahi forest

Occurs on the tephra mantled plateau surrounding Lake Taupo, and on the ringplains of the Tongariro and Taranaki volcanoes. Conifers are dominant with abundant rimu, miro and totara—matai and kahikatea are also widespread, reaching their greatest abundance on sites with sandy and poorly drained soils respectively. Kamahi is widespread in the understorey along with tawa, although the latter is excluded from depressions where cold air ponding leads to intense frost. Other widespread understorey species include hinau, black and white maires, fuchsia and mahoe. 


\section{Appendix VII \\ Landscape metrics used in Study III}

\section{Notes:}

- A verbatim copy of the standard definitions of landscape metrics has been provided here based upon McGarigal, 2015. Written permission for this was obtained from Professor Kevin McGarigal on 4 November 2017 via email.

- The area-weighted mean of CORE, CAI, ENN, PROX, was used to quantify and measure the study area at the class level. According to McGarigal (2015) 'the area-weighted mean of any patch metric (i.e., distribution statistics at the class and landscape level) weights each patch by its proportional area representation'.

MN (Mean) equals the sum, across all patches in the landscape, of the corresponding patch metric values, divided by the total number of patches. $\mathrm{MN}$ is given in the same units as the corresponding patch metric.

AM (area-weighted mean) equals the sum, across all patches in the landscape, of the corresponding patch metric value multiplied by the proportional abundance of the patch [i.e., patch area $\left(\mathrm{m}^{2}\right)$ divided by the sum of patch areas]. Note, the proportional abundance of each patch is determined from the sum of patch areas rather than the total landscape area, because the latter may include internal background area not associated with any patch.

\section{Total Class Area (CA)}

Description: CA equals the sum of the areas $\left(\mathrm{m}^{2}\right)$ of all patches of the corresponding patch type, divided by 10,000 (to convert to hectares); that is, total class area.

Units: Hectares

Range: $\mathrm{CA}>0$, without limit.

$\mathrm{CA}$ approaches 0 as the patch type becomes increasing rare in the landscape. CA $=$ TA when the entire landscape consists of a single patch type; that is, when the entire image is comprised of a single patch.

Comments: Class area is a measure of landscape composition; specifically, how much of the landscape is comprised of a particular patch type. In addition to its direct interpretive value, class area is used in the computations for many of the class and landscape metrics.

\section{Percentage of Landscape (PLAND)}

Description: PLAND equals the sum of the areas $\left(\mathrm{m}^{2}\right)$ of all patches of the corresponding patch type, divided by total landscape area $\left(\mathrm{m}^{2}\right)$, multiplied by 100 (to convert to a percentage); in other words, PLAND equals the percentage the landscape comprised of the corresponding patch type. Note, total landscape area (A) includes any internal background present.

Units: percent

Range: $0<$ PLAND $\leq 100$

PLAND approaches 0 when the corresponding patch type (class) becomes increasingly rare in the landscape. PLAND $=100$ when the entire landscape consists of a single patch type; that is, when the entire image is comprised of a single patch.

Comments: Percentage of landscape quantifies the proportional abundance of each patch type in the landscape. Like total class area, it is a measure of landscape composition important in many ecological applications. However, because PLAND is a relative measure, it may be a more appropriate measure of landscape composition than class area for comparing among landscapes of varying sizes. 


\section{Number of Patches (NP)}

Description: NP equals the number of patches of the corresponding patch type (class).

Units: None

Range: $\mathrm{NP} \geq 1$, without limit

$\mathrm{NP}=1$ when the landscape contains only 1 patch of the corresponding patch type; that is, when the class consists of a single patch.

Comments: Number of patches of a particular patch type is a simple measure of the extent of subdivision or fragmentation of the patch type. Although the number of patches in a class may be fundamentally important to a number of ecological processes, often it has limited interpretive value by itself because it conveys no information about area, distribution, or density of patches. Of course, if total landscape area and class area are held constant, then number of patches conveys the same information as patch density or mean patch size and may be a useful index to interpret. Number of patches is probably most valuable, however, as the basis for computing other, more interpretable, metrics. Note that the choice of the 4neighbour or 8-neighbor rule for delineating patches will have an impact on this metric.

\section{Edge Density (ED)}

ED equals the sum of the lengths (m) of all edge segments involving the corresponding patch type, divided by the total landscape area $\left(\mathrm{m}^{2}\right)$, multiplied by 10,000 (to convert to hectares). If a landscape border is present, ED includes landscape boundary segments involving the corresponding patch type and representing 'true' edge only (i.e., abutting patches of different classes). If a landscape border is absent, ED includes a user-specified proportion of landscape boundary segments involving the corresponding patch type. Regardless of whether a landscape border is present or not, ED includes a userspecified proportion of internal background edge segments involving the corresponding patch type. Note, total landscape area (A) includes any internal background present.

Units: metres per hectare

Range: $\mathrm{ED} \geq 0$, without limit

$\mathrm{ED}=0$ when there is no class edge in the landscape; that is, when the entire landscape and landscape border, if present, consists of the corresponding patch type and the user specifies that none of the landscape boundary and background edge be treated as edge.

Comments: Edge density has the same utility and limitations as Total Edge (see Total Edge description), except that edge density reports edge length on a per unit area basis that facilitates comparison among landscapes of varying size.

\section{Area (AREA)}

Description: AREA equals the area $\left(\mathrm{m}^{2}\right)$ of the patch, divided by 10,000 (to convert to hectares).

Units: Hectares

Range: AREA $>0$, without limit

The range in AREA is limited by the grain and extent of the image; in a particular application, AREA may be further limited by the specification of a minimum patch size that is larger than the grain.

Comments: The area of each patch comprising a landscape mosaic is perhaps the single most important and useful piece of information contained in the landscape. Not only is this information the basis for many of the patch, class, and landscape indices, but patch area has a great deal of ecological utility in its own right. Note that the choice of the 4-neighbor or 8neighbor rule for delineating patches will have an impact on this metric.

\section{Radius of Gyration (GYRATE)}

Description: GYRATE equals the mean distance (m) between each cell in the patch and the patch centroid.

Units: Metres

Range: GYRATE $\geq 0$, without limit

GYRATE $=0$ when the patch consists of a single cell and increases without limit as the patch increases in extent. GYRATE achieves its maximum value when the patch comprises the entire landscape.

Comments: Radius of gyration is a measure of patch extent; thus it is effected by both patch size and patch compaction. Note that the choice of the 4-neighbor or 8-neighbor rule for delineating patches will have an impact on this metric. 


\section{Total Core Area (TCA)}

TCA equals the sum of the core areas of each patch $\left(\mathrm{m}^{2}\right)$ of the corresponding patch type, divided by 10,000 (to convert to hectares).

Units: hectares

Range: TCA $\geq 0$, without limit

TCA $=0$ when every location within each patch of the corresponding patch type is within the specified depth-of-edge distance(s) from the patch perimeters. TCA approaches total class area (CA) as the specified depth-of-edge distance(s) decreases and as patch shapes are simplified.

Comments: Total core area is defined the same as core area (CORE) at the patch level (see Core Area), but here core area is aggregated (summed) over all patches of the corresponding patch type.

\section{Core Area Percentage of Landscape (CPLAND)}

Description: CPLAND equals the sum of the core areas of each patch $\left(\mathrm{m}^{2}\right)$ of the corresponding patch type, divided by total landscape area $\left(\mathrm{m}^{2}\right)$, multiplied by 100 (to convert to a percentage); in other words, CPLAND equals the percentage the landscape comprised of core area of the corresponding patch type. Note, total landscape area (A) includes any internal background present.

Units: percent

Range: $0 \leq$ CPLAND $<100$

CPLAND approaches 0 when core area of the corresponding patch type (class) becomes increasingly rare in the landscape, because of increasing smaller patches and/or more convoluted patch shapes. CPLAND approaches 100 when the entire landscape consists of a single patch type (i.e., when the entire image is comprised of a single patch) and the specified depthof-edge distance(s) approaches zero.

Comments: Core area percentage of landscape is defined the same as core area (CORE) at the patch level (see Core Area), but here core area is aggregated (summed) over all patches of the corresponding patch type and computed as a percentage of the total landscape area, which facilitates comparison among landscape of varying size.

\section{Core Area (CORE)}

Description: CORE equals the area $\left(\mathrm{m}^{2}\right)$ within the patch that is further than the specified depth-of-edge distance from the patch perimeter, divided by 10,000 (to convert to hectares). Edge segments along the landscape boundary are treated like background (as specified in the edge depth file) unless a landscape border is present, in which case the boundary edge types are made explicit by the information in the border.

Units: Hectares

Range: $\mathrm{CORE} \geq 0$, without limit

CORE $=0$ when every location within the patch is within the specified depth-of edge distance from the patch perimeter. CORE approaches AREA as the specified depth-of-edge distance(s) decreases and as patch shape is simplified.

Comments: Core area represents the area in the patch greater than the specified depth-of-edge distance from the perimeter. Note, that a single depth-of-edge distance can be used for all edges or the user can specify a edge depth file that provides unique distances for each pairwise combination of patch types.

\section{Core Area Index (CAI)}

Description: CAI equals the patch core area $\left(\mathrm{m}^{2}\right)$ divided by total patch area $\left(\mathrm{m}^{2}\right)$, multiplied by 100 (to convert to a percentage); in other words, CAI equals the percentage of a patch that is core area.

Units: percent

Range: $0 \leq \mathrm{CAI}<100$

$\mathrm{CAI}=0$ when $\mathrm{CORE}=0$ (i.e., every location within the patch is within the specified depth-of-edge distance(s) from the patch perimeter); that is, when the patch contains no core area. CAI approaches 100 when the patch, because of size, shape, and edge width, contains mostly core area.

Comments: Core area index is a relative index that quantifies core area as a percentage of patch area (i.e., the percentage of the patch that is comprised of core area). 


\section{Proximity Index (PROX)}

Description: PROX equals the sum of patch area $\left(\mathrm{m}^{2}\right)$ divided by the nearest edge-to-edge distance squared $\left(\mathrm{m}^{2}\right)$ between the patch and the focal patch of all patches of the corresponding patch type whose edges are within a specified distance (m) of the focal patch. Note, when the search buffer extends beyond the landscape boundary, only patches contained within the landscape are considered in the computations. In addition, note that the edge-to-edge distances are from cell center to cell center.

Units: None

Range: $\mathrm{PROX} \geq 0$

$\mathrm{PROX}=0$ if a patch has no neighbors of the same patch type within the specified search radius. PROX increases as the neighborhood (defined by the specified search radius) is increasingly occupied by patches of the same type and as those patches become closer and more contiguous (or less fragmented) in distribution. The upper limit of PROX is affected by the search radius and the minimum distance between patches.

Comments: Proximity index was developed by Gustafson and Parker (1992) and considers the size and proximity of all patches whose edges are within a specified search radius of the focal patch. Note that FRAGSTATS uses the distance between the focal patch and each of the other patches within the search radius, similar to the isolation index of Whitcomb et al. (1981), rather than the nearest-neighbor distance of each patch within the search radius (which could be to a patch other than the focal patch), as in Gustafson and Parker (1992). The index is dimensionless (i.e., has no units) and therefore the absolute value of the index has little interpretive value; instead it is used as a comparative index.

\section{Euclidean Nearest-Neighbor Distance (ENN)}

Description: ENN equals the distance (m) to the nearest neighboring patch of the same type, based on shortest edge-to-edge distance. Note that the edge-to-edge distances are from cell center to cell center.

Units: Meters

Range: $\mathrm{ENN}>0$, without limit

ENN approaches 0 as the distance to the nearest neighbor decreases. The minimum ENN is constrained by the cell size and is equal to twice the cell size when the 8-neighbor patch rule is used or the distance between diagonal neighbors when the 4neighbor rule is used. The upper limit is constrained by the extent of the landscape. ENN is undefined and reported as "N/A" in the "basename". patch file if the patch has no neighbors (i.e., no other patches of the same class).

Comments: Euclidean nearest-neighbor distance is perhaps the simplest measure of patch context and has been used extensively to quantify patch isolation. Here, nearest neighbor distance is defined using simple Euclidean geometry as the shortest straight-line distance between the focal patch and its nearest neighbor of the same class.

\section{Patch Cohesion Index (COHESION)}

Description: COHESION equals 1 minus the sum of patch perimeter (in terms of number of cell surfaces) divided by the sum of patch perimeter times the square root of patch area (in terms of number of cells) for patches of the corresponding patch type, divided by 1 minus 1 over the square root of the total number of cells in the landscape, multiplied by 100 to convert to a percentage. Note, total landscape area (Z) excludes any internal background present.

Units: None

Range: $0<$ COHESION $<100$

COHESION approaches 0 as the proportion of the landscape comprised of the focal class decreases and becomes increasingly subdivided and less physically connected. COHESION increases monotonically as the proportion of the landscape comprised of the focal class increases until an asymptote is reached near the percolation threshold (see background discussion). COHESION is given as 0 if the landscape consists of a single non-background cell.

Comments: Patch cohesion index measures the physical connectedness of the corresponding patch type. Below the percolation threshold, patch cohesion is sensitive to the aggregation of the focal class. Patch cohesion increases as the patch type becomes more clumped or aggregated in its distribution; hence, more physically connected. Above the percolation threshold, patch cohesion does not appear to be sensitive to patch configuration (Gustafson 1998).

14. Conectance Index (CONNECT)

Description: CONNECT equals the number of functional joinings between all patches of the corresponding patch type (sum of $c_{i j k}$ where $c_{i j k}=0$ if patch $\mathrm{j}$ and $\mathrm{k}$ are not within the specified distance of each other and $c_{\mathrm{ijk}}=1$ if patch $\mathrm{j}$ and $\mathrm{k}$ are 
within the specified distance), divided by the total number of possible joinings between all patches of the corresponding patch type, multiplied by 100 to convert to a percentage.

Units: percent

Range: $0 \leq$ CONNECT $\leq 100$

CONNECT $=0$ when either the focal class consists of a single patch or none of the patches of the focal class are "connected" (i.e., within the user-specified threshold distance of another patch of the same type). CONNECT = 100 when every patch of the focal class is "connected."

Comments: Connectance is defined on the number of functional joinings between patches of the corresponding patch type, where each pair of patches is either connected or not based on a user-specified distance criterion. Connectance is reported as a percentage of the maximum possible connectance given the number of patches. Note, connectance can be based on either Euclidean distance or functional distance, as described elsewhere (see Isolation/Proximity Metrics).

\section{Shape Index (SHAPE)}

Description: SHAPE equals patch perimeter (m) divided by the square root of patch area (m2), adjusted by a constant to adjust for a square standard.

Units: None

Range: $1 \geq$ without limit

SHAPE $=1$ when the patch is square and increases without limit as patch shape becomes more irregular.

Comments: Shape index corrects for the size problem of the perimeter-area ratio index by adjusting for a square standard and, as a result, is the simplest and perhaps most straightforward measure of shape complexity. 